\title{
Pentakis(trifluoromethyl)phenyl, a Sterically Crowded and Electron-withdrawing Group: Synthesis and Acidity of Pentakis(trifluoromethyl)benzene, -toluene, -phenol, and -aniline
}

\section{Supporting Information}

Agnes Kütt, ${ }^{1}$ Valeria Movchun, ${ }^{2}$ Toomas Rodima, ${ }^{1}$ Timo Dansauer, ${ }^{3}$ Eduard B. Rusanov, ${ }^{2}$ Ivo Leito, ${ }^{*}, 1$ Ivari Kaljurand, ${ }^{1}$ Juta Koppel, ${ }^{1}$ Viljar Pihl, ${ }^{1}$ Ivar Koppel, ${ }^{1}$ Gea Ovsjannikov, ${ }^{1}$ Lauri Toom, ${ }^{4}$ Masaaki Mishima, ${ }^{5}$ Maurice Medebielle, ${ }^{6}$ Enno Lork, ${ }^{3}$ GerdVolker Röschenthaler, ${ }^{* 3}$ Ilmar A. Koppel, ${ }^{*, 1}$ Alexander A. Kolomeitsev, ${ }^{*, 3, *}$

ivo.leito@ut.ee,ilmar.koppel@ut.ee,gvr@chemie.uni-bremen.de, kolomeit@uni-bremen.de

${ }^{1}$ University of Tartu, Institute of Chemistry, 2 Jakobi St. 51014 Tartu, Estonia

${ }^{2}$ Institute of Organic Chemistry, Ukrainian National Academy of Sciences, 5 Murmanskaya St., Kiev 02094, Ukraine

${ }^{3}$ Institute of Inorganic \& Physical Chemistry, University of Bremen, Leobener St. D-28334 Bremen, Germany

${ }^{4}$ University of Tartu, Institute of Technology, 1 Nooruse St. 50411 Tartu, Estonia

${ }^{5}$ Kyushu University, Institute for Materials Chemistry and Engineering, Hakozaki, Higashi-ku, Fukuoka 812-8581, Japan

${ }^{6}$ Université Paris 7 Denis Diderot, Electrochimie Moleculaire, UMR CNRS 7591 Case courrier 7107, 2 place Jussieu, F-75251 Paris Cedex 05, France ${ }^{\dagger}$

\footnotetext{
${ }^{\dagger}$ Present address: Universite Claude Bernard Lyon 1, "Institut de Chimie et Biochimie Moléculaires et Supramoléculaires", 43 bd du 11 Novembre 1918, 69622 Villeurbanne, FRANCE.

\$ Present address: Hansa Fine Chemicals GmbH, Fahrenheitstrasse 1, D-28359, Bremen, GERMANY.
} 


\section{Table of Contents}

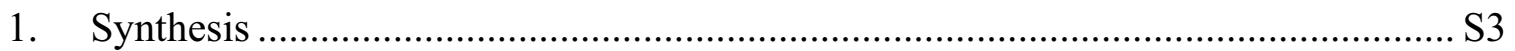

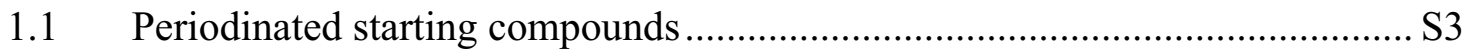

$1.2 \quad 2,3,4,6$-tetrakis(trifluoromethyl)phenyl Compounds ....................................... S4

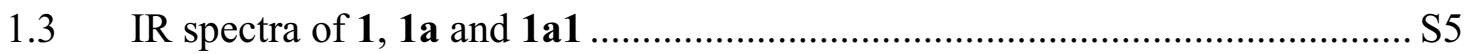

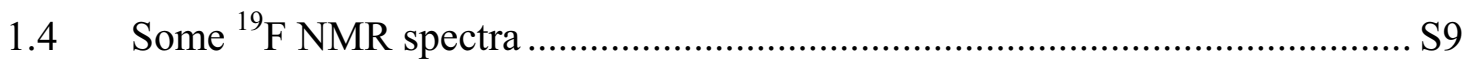

2. Deprotonation of Pentakis(trifluoromethyl)benzene (3)........................................ S13

3. Determination of Acidities in Acetonitrile (AN) and in the Gas Phase.................. S14

3.1 Detailed Description of Determination of $\Delta \mathrm{p} K_{\mathrm{a}}$ Values in AN ...................... S14

3.2 Results of Gas-phase Acidity Measurements ………………….................... S20

3.3 Acidities of Pentakis(trifluoromethyl)phenyl Derivatives in the Gas Phase,

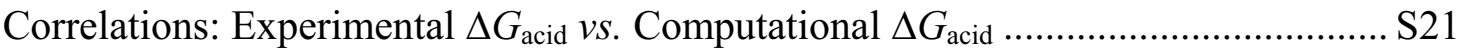

3.4 Calculation of $\Delta G$ and $\Delta H$ Values of Isodesmic Reactions .......................... S24

3.5 Calculation of Additivities of $\mathrm{p} K_{\mathrm{a}}$ Values and $\Delta G_{\text {acid }}$ Values ......................... S32

3.6 The Results of the Acidity Calculations of Some Pentakis(trifluoromethyl) Substituted $\mathrm{CH}, \mathrm{OH}, \mathrm{NH}$ Acids and Related Compouns at DFT B3LYP 6-311+G** level S35

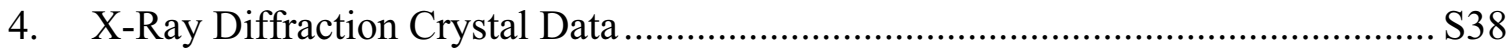

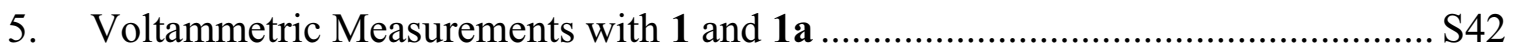

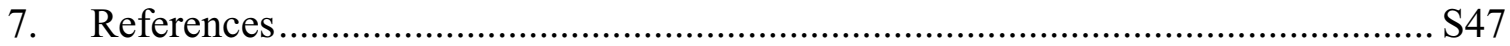

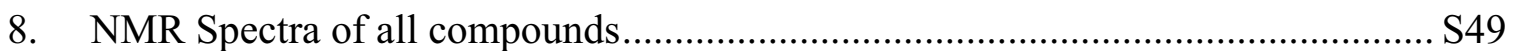

9. Optimized Geometries Obtained from the Computations ……………………...... S87 


\section{Synthesis}

\subsection{Periodinated starting compounds}

Hexaiodobenzene. In addition to the literature method, $\mathrm{C}_{6} \mathrm{I}_{6}$ was washed carefully with hot nitrobenzene and a part of $\mathrm{C}_{6} \mathrm{I}_{6}$ was recrystallized from nitrobenzene. Between these two batches of compounds there were no differences in melting points, $\mathrm{mp} \geq 380{ }^{\circ} \mathrm{C}$ dec (lit. ${ }^{1} \mathrm{mp} 380-419{ }^{\circ} \mathrm{C}$ ); ${ }^{13} \mathrm{C}$ NMR (90.5 MHz, DMSO-d $\left.{ }_{6}\right) \delta$ : $117.20\left(\mathrm{~s}, \mathrm{C}_{\mathrm{Ar}}\right.$ ); EIMS $\mathrm{m} / \mathrm{z}$ (relative intensity, ion): $833\left(100 \%, \mathrm{M}^{+}\right), 707\left(12 \%, \mathrm{M}^{+}-\mathrm{I}\right), 580\left(16 \%, \mathrm{M}^{+}-2 \mathrm{I}\right), 453(8 \%$, $\left.\mathrm{M}^{+}-3 \mathrm{I}\right), 326$ (14\%, $\left.\mathrm{M}^{+}-4 \mathrm{I}\right), 199\left(10 \%, \mathrm{M}^{+}-5 \mathrm{I}\right), 72$ (4\%, $\left.\mathrm{M}^{+}-6 \mathrm{I}\right)$.

Pentaiodotoluene. The conditions for the permercuration of sodium $p$-tolylsulphinate ${ }^{2}$ $\left(180^{\circ} \mathrm{C}\right)$ and followed by periodination (ambient temperature, 3 days) seemed not to be appropriate to get pure pentaiodotoluene. Therefore reaction parameters were changed somewhat to obtain the desired product by the following procedure: mercury(II) oxide $(0.11 \mathrm{~mol}, 22.7 \mathrm{~g})$ was dissolved in in refluxing trifluoroacetic acid $(22.5 \mathrm{~mL}, 0.29 \mathrm{~mol}$, $33 \mathrm{~g})$. The solution was then heated up to $210^{\circ} \mathrm{C}$ and sodium $p$-tolylsulphinate $(15 \mathrm{mmol}$, $2.0 \mathrm{~g}$ ) was added. The mixture was heated for $20 \mathrm{~min}$ at $200^{\circ} \mathrm{C}$ and then cooled. The solid permercurated compound was dissolved in DMF $(150 \mathrm{~mL})$, poured into a solutionof iodine $(0.14 \mathrm{~mol}, 35 \mathrm{~g})$ and sodium iodide $(0.21 \mathrm{~mol}, 35 \mathrm{~g})$ in DMF $(75 \mathrm{~mL})$ and allowed to be stirred at room temperature for 10 days and at $50^{\circ} \mathrm{C}$ for $12 \mathrm{~h}$. The precipitated pentaiodotoluene was filtered, washed with $20 \% \mathrm{KBr}$ aqueous solution, water and methanol and dried in vacuum. Recrystallization from toluene yielded yellow crystals (4.02 mmol, $2.9 \mathrm{~g}, 27 \%$ ), mp $328-333^{\circ} \mathrm{C}$ dec (lit. ${ }^{2} 306-309^{\circ} \mathrm{C}$ ); EIMS (\% relative intensity, ion) $m / z$ : $722\left(100 \%, \mathrm{M}^{+}\right), 595\left(14 \%, \mathrm{M}^{+}-\mathrm{I}\right), 468\left(22 \%, \mathrm{M}^{+}-2 \mathrm{I}\right), 341(21 \%$, $\left.\mathrm{M}^{+}-3 \mathrm{I}\right), 214$ (11\%, $\left.\mathrm{M}^{+}-4 \mathrm{I}\right), 87\left(13 \%, \mathrm{M}^{+}-5 \mathrm{I}\right)$.

Pentaiodochlorobenzene. The compound was prepared exactly according to the literature method. ${ }^{1} \mathrm{Mp} \geq 315^{\circ} \mathrm{C}$ dec (lit. ${ }^{1} \mathrm{mp} 356-358{ }^{\circ} \mathrm{C}$ ); EIMS (\% relative intensity, ion) $\mathrm{m} / \mathrm{z}$ : $741\left(100 \%, \mathrm{M}^{+}\right), 615\left(13 \%, \mathrm{M}^{+}-\mathrm{I}\right), 488\left(16 \%, \mathrm{M}^{+}-2 \mathrm{I}\right), 371\left(4 \%, \mathrm{M}^{2+}\right), 361(8 \%$, $\left.\mathrm{M}^{+}-3 \mathrm{I}\right), 326$ (7\%, M+3I-Cl), 234 (8\%, M+4I), 199 (3\%, M+4I-Cl), 107 (4\%, $\left.\mathrm{M}^{+}-5 \mathrm{I}\right)$, $72\left(2.5 \%, \mathrm{M}^{+}-5 \mathrm{I}-\mathrm{Cl}\right)$.

4-trifluoromethyl-2,3,6-triodochlorobenzene was prepared according to a literature method. ${ }^{1}$ In conc. $\mathrm{H}_{2} \mathrm{SO}_{4}(60 \mathrm{~mL})$ periodic acid $(17 \mathrm{mmol}, 3.9 \mathrm{~g})$ was dissolved. To the 
clear solution $\mathrm{I}_{2}(51.4 \mathrm{mmol}, 13.05 \mathrm{~g})$ was added and the mixture stirred at room temperature for $40 \mathrm{~min}$. To the cooled mixture $\left(0^{\circ} \mathrm{C}\right)$ of p-chlorobenzotrifluoride $(20$ mmol, $3.6 \mathrm{~g}, 2.7 \mathrm{~mL}$ ) was slowly added. The temperature of the mixture was allowed to rise to ambient and it was still stirred for $24 \mathrm{~h}$ at room temperature and another $24 \mathrm{~h}$ at $90{ }^{\circ} \mathrm{C}$. The cold mixture was poured onto ice. The precipitate was filtered and washed with methanol $(150 \mathrm{~mL})$. To the methanolic washings water $(75 \mathrm{~mL})$ was added. Formed precipitate, the raw product, was filtered and washed with 1:1 mixture of $\mathrm{MeOH} / \mathrm{H}_{2} \mathrm{O}$. Recrystallization from $\mathrm{MeOH}$ and/or $\mathrm{MeCN}$ gave a light yellowish crystals. Yield 31\% (11.65 mmol, $6.5 \mathrm{~g}$ ), mp 103-104 ${ }^{\circ} \mathrm{C} ;{ }^{1} \mathrm{H}$ NMR $\left(200.1 \mathrm{MHz}, \mathrm{CDCl}_{3}\right)$ ): 8.08 (s, ArH, $\left.1 \mathrm{H}\right)$; ${ }^{19} \mathrm{~F}$ NMR $\left(188.3 \mathrm{MHz}, \mathrm{CDCl}_{3}\right) \delta:-64.23\left(\mathrm{~s}, p-\mathrm{CF}_{3}, 3 \mathrm{~F}\right) ;{ }^{13} \mathrm{C} \mathrm{NMR}\left(50.3 \mathrm{MHz}, \mathrm{CDCl}_{3}\right) \delta$ : $146.42\left(\mathrm{~s}, 1-\mathrm{C}_{\mathrm{Ar}}\right), 138.20\left(\mathrm{q},{ }^{3} J_{\mathrm{C}-\mathrm{F}}=6.06 \mathrm{~Hz}, 5-\mathrm{C}_{\mathrm{Ar}}\right), 134.62\left(\mathrm{q},{ }^{2} J_{\mathrm{C}-\mathrm{F}}=31.11 \mathrm{~Hz}, 4-\mathrm{C}_{\mathrm{Ar}}\right)$, $120.54\left(\mathrm{q},{ }^{1} J_{\mathrm{C}-\mathrm{F}}=275.09 \mathrm{~Hz}, 4-\mathrm{CF}_{3}\right), 119.01\left(\mathrm{~s}, 2-\mathrm{C}_{\mathrm{Ar}}\right), 108.15\left(\mathrm{~s}, 3-\mathrm{C}_{\mathrm{Ar}}\right), 95.32(\mathrm{~s}, 6-$ $\mathrm{C}_{\mathrm{Ar}}$ ); EIMS (\% relative intensity, ion) $m / z$ : $558\left(100 \%, \mathrm{M}^{+}\right), 431\left(16 \%, \mathrm{M}^{+}-\mathrm{I}\right), 304(13 \%$, $\left.\mathrm{M}^{+}-2 \mathrm{I}\right), 177\left(21 \%, \mathrm{M}^{+}-3 \mathrm{I}\right)$; HRMS-EI $m / z: \mathrm{M}^{+}$calcd for $\mathrm{C}_{7} \mathrm{HF}_{3} \mathrm{ClI}_{3}$ 557.68477; found 557.68485 .

\subsection{2,3,4,6-tetrakis(trifluoromethyl)phenyl Compounds}

2,3,4,6-tetrakis(trifluoromethyl)chlorobenzene. According to general procedure to pregenerate $\mathrm{CF}_{3} \mathrm{Cu}, \mathrm{CuBr}$ (36.8 mmol, $5.28 \mathrm{~g}$ ), $\mathrm{CF}_{3} \mathrm{SiMe}_{3}$ (29.0 mmol, $4.13 \mathrm{~g}$ ), $\mathrm{KF}$ (29.25 mmol, $1.70 \mathrm{~g})$, DMF $(30 \mathrm{~mL})$ and NMP $(6 \mathrm{~mL})$ were mixed. of 2,3,4,6-tetrakis(trifluoromethyl)chlorobenzene (7.34 mmol, $4.5 \mathrm{~g})$ and additional NMP $(30 \mathrm{~mL})$ were added. The reaction and further workup were carried out the same way as described for 4 . For further purification differential distillation was performed. The compound was yellowish liquid. Yield 36\% (2.6 mmol, $1.0 \mathrm{~g}$ ), bp 80-81 ${ }^{\circ} \mathrm{C}, 15$ mbar. ${ }^{1} \mathrm{H}$ NMR $\left(200.1 \mathrm{MHz}, \mathrm{CDCl}_{3}\right) \delta$ : $8.10(\mathrm{~s}, \mathrm{ArH}, 1 \mathrm{H}) ;{ }^{19} \mathrm{~F}$ NMR $\left(188.3 \mathrm{MHz}, \mathrm{CDCl}_{3}\right) \delta:-54.28$ (qq, ${ }^{5} J_{\mathrm{F}-\mathrm{F}}=15.7,{ }^{5} J_{\mathrm{F}-\mathrm{F}}=15.7$ $\left.\mathrm{Hz}, 3-\mathrm{CF}_{3}, 3 \mathrm{~F}\right),-56.69\left(\mathrm{q},{ }^{5} J_{\mathrm{FF}}=15.7 \mathrm{~Hz}, 4-\mathrm{CF}_{3}, 3 \mathrm{~F}\right),-58.49\left(\mathrm{q},{ }^{5} J_{\mathrm{FF}}=15.7 \mathrm{~Hz}, 1-\mathrm{CF}_{3}\right.$, 3F), -64.11 (s, 6- $\left.\mathrm{CF}_{3}, 3 \mathrm{~F}\right) ;{ }^{13} \mathrm{C}$ NMR (50.3 MHz, $\left.\mathrm{CDCl}_{3}\right) \delta: 138.2\left(\mathrm{~s}, 1-\mathrm{C}_{\mathrm{Ar}}\right), 134.3$ (q, ${ }^{2} J_{\mathrm{C}-\mathrm{F}}=37.5 \mathrm{~Hz}, 2$ - or $4-$ or $\left.6-\mathrm{C}_{\mathrm{Ar}}\right), 134.2\left(\mathrm{qm},{ }^{2} J_{\mathrm{C}-\mathrm{F}}=33.0 \mathrm{~Hz}, 3-\mathrm{C}_{\mathrm{Ar}}\right), 132.8\left(\mathrm{qm},{ }^{2} J_{\mathrm{C}-\mathrm{F}}=\right.$ $33.9 \mathrm{~Hz}, 2$ - or 4- or 6- $\mathrm{C}_{\mathrm{Ar}}$ ), $130.3\left(\mathrm{qm},{ }^{2} J_{\mathrm{C}-\mathrm{F}}=35.5 \mathrm{~Hz}, 2\right.$ - or 4- or 6- $\mathrm{C}_{\mathrm{Ar}}$ ), 129.7 (sept, ${ }^{3} J_{\mathrm{C}-}$ $\left.\mathrm{F}=5.9 \mathrm{~Hz}, 5-\mathrm{C}_{\mathrm{Ar}}\right), 121.9\left(\mathrm{q},{ }^{1} J_{\mathrm{C}-\mathrm{F}}=275.1 \mathrm{~Hz}, 4-\right.$ or $\left.6-\mathrm{CF}_{3}\right), 121.4\left(\mathrm{q},{ }^{1} J_{\mathrm{C}-\mathrm{F}}=277.2 \mathrm{~Hz}, 4-\right.$ or 6-CF 3 ), $121.2\left(\mathrm{q},{ }^{1} J_{\mathrm{C}-\mathrm{F}}=274.7 \mathrm{~Hz}, 2-\right.$ or $\left.3-\mathrm{CF}_{3}\right), 121.0\left(\mathrm{q},{ }^{1} J_{\mathrm{C}-\mathrm{F}}=276.8 \mathrm{~Hz}, 2-\right.$ or $3-$ 
$\left.\mathrm{CF}_{3}\right)$; EIMS $m / z$ (\% relative intensity, ion): $348\left(100 \%, \mathrm{M}^{+}\right), 365\left(78 \%, \mathrm{M}^{+}-\mathrm{F}\right), 349(47 \%$, $\left.\mathrm{M}^{+}-\mathrm{Cl}\right), 315\left(94 \%, \mathrm{M}^{+}-\mathrm{CF}_{3}\right)$. HRMS-EI: $m / z: \mathrm{M}^{+}$calcd for $\mathrm{C}_{10} \mathrm{HF}_{12} \mathrm{Cl}, 383.95697$; found, 383.95825 .

Tetraethylammonium dicyano[2,3,4,6-tetrakis(trifluoromethyl)phenyl]methanide.

To the $\mathrm{NaH}\left(2.9 \mathrm{mmol}, 0.51 \mathrm{~g}\right.$, dissolved in $5 \mathrm{~mL}$ of dry THF) and of $\mathrm{CH}_{2}(\mathrm{CN})_{2}(1.67$ mmol, $0.11 \mathrm{~g}$, dissolved in $1 \mathrm{~mL}$ of dry THF) at $-80{ }^{\circ} \mathrm{C} \quad 2,3,4,6-$ tetrakis(trifluoromethyl)chlorobenzene $(1.33 \mathrm{mmol}, 0.51 \mathrm{~g}$, dissolved in $1 \mathrm{~mL}$ of dry THF) was added. The following procedure was performed the same way as the synthesis of 7a. $\mathrm{Et}_{4} \mathrm{NCl}(1.5 \mathrm{mmol}, 0.25 \mathrm{~g})$ was added to get instead of the sodium salt a tetraethylammonium salt. Purified product $(0.73 \mathrm{mmol}, 0.39 \mathrm{~g})$ was obtained. Yield: $54 \%$, mp 113-116 ${ }^{\circ} \mathrm{C} ;{ }^{1} \mathrm{H}$ NMR (400.1 MHz, DMSO-d $)$ ) $8.86(\mathrm{~s}, \mathrm{ArH}, 1 \mathrm{H}), 3.49\left(\mathrm{q},{ }^{3} J_{\mathrm{HH}}=\right.$ $\left.7.3 \mathrm{~Hz}, \mathrm{CH}_{2}, 8 \mathrm{H}\right), 1.39$ (qt, $\left.{ }^{3} J_{\mathrm{HH}}=7.3 \mathrm{~Hz},{ }^{3} \mathrm{~J}_{\mathrm{H}-}{ }^{14} \mathrm{~N}=1.9 \mathrm{~Hz}, \mathrm{CH}_{3}, 12 \mathrm{H}\right) ;{ }^{19} \mathrm{~F}$ NMR $(188.3$ $\mathrm{MHz}$, Acetone-d 6 ) $\delta:-55.11\left(\mathrm{qq},{ }^{5} J_{\mathrm{F}-\mathrm{F}}=15.4,{ }^{5} J_{\mathrm{F}-\mathrm{F}}=15.4 \mathrm{~Hz}, 3-\mathrm{CF}_{3}, 3 \mathrm{~F}\right),-55.66\left(\mathrm{q},{ }^{5} J_{\mathrm{F}-\mathrm{F}}\right.$ $\left.=15.4 \mathrm{~Hz}, 4-\mathrm{CF}_{3}, 3 \mathrm{~F}\right),-58.29\left(\mathrm{q},{ }^{5} J_{\mathrm{F}-\mathrm{F}}=15.4 \mathrm{~Hz}, 1-\mathrm{CF}_{3}, 3 \mathrm{~F}\right),-60.85\left(\mathrm{~s}, 6-\mathrm{CF}_{3}, 3 \mathrm{~F}\right) ;{ }^{13} \mathrm{C}$ NMR (100.6 MHz, Acetone-d $\left.\mathrm{d}_{6}\right) \delta: 146.0\left(\mathrm{~m}, 1-\mathrm{C}_{\mathrm{Ar}}\right) 134.1$ (qm, ${ }^{2} J_{\mathrm{C}-\mathrm{F}}=34 \mathrm{~Hz}, 3-\mathrm{C}_{\mathrm{Ar}}$ ), $129.0\left(\mathrm{qm},{ }^{3} J_{\mathrm{C}-\mathrm{F}}=6.9,6.8 \mathrm{~Hz}, 5-\mathrm{C}_{\mathrm{Ar}}\right), 125.2\left(\mathrm{qm},{ }^{2} J_{\mathrm{C}-\mathrm{F}}=31.1 \mathrm{~Hz}, 6-\mathrm{C}_{\mathrm{Ar}}\right), 124.4\left(\mathrm{q},{ }^{1} J_{\mathrm{C}-\mathrm{F}}\right.$

$=271.6 \mathrm{~Hz}, 4-$ or $\left.6-\mathrm{CF}_{3}\right), 124.2\left(\mathrm{q},{ }^{1} J_{\mathrm{C}-\mathrm{F}}=273.2 \mathrm{~Hz}, 2-\right.$ or $\left.3-\mathrm{CF}_{3}\right), 124.1\left(\mathrm{q},{ }^{1} J_{\mathrm{C}-\mathrm{F}}=273.2\right.$ $\mathrm{Hz}, 4-$ or $\left.6-\mathrm{CF}_{3}\right), 123.2$ (q, ${ }^{1} J_{\mathrm{C}-\mathrm{F}}=274.1 \mathrm{~Hz}, 2-$ or $\left.3-\mathrm{CF}_{3}\right), 122.7(\mathrm{~s}, \mathrm{CN}), 120.9\left(\mathrm{qm},{ }^{2} J_{\mathrm{C}-\mathrm{F}}\right.$ $\left.=34 \mathrm{~Hz}, 2-\mathrm{C}_{\mathrm{Ar}}\right), 117.5\left(\mathrm{qm},{ }^{2} J_{\mathrm{C}-\mathrm{F}}=34.1 \mathrm{~Hz}, 4-\mathrm{C}_{\mathrm{Ar}}\right), 79.2\left(\mathrm{~s}, \mathrm{C}(\mathrm{CN})_{2}\right), 53.1\left(\mathrm{t},{ }^{1} J_{\mathrm{C}-}{ }^{14} \mathrm{~N}=3.1\right.$ $\left.\mathrm{Hz}, \mathrm{CH}_{2}\right), 7.7\left(\mathrm{~s}, \mathrm{CH}_{3}\right)$; HRMS-ESI $\mathrm{m} / z$ : $\mathrm{M}^{-}$calcd for $\mathrm{C}_{13} \mathrm{HN}_{2} \mathrm{~F}_{12}, 412.99533$; found, 412.99533; $\mathrm{Et}_{4} \mathrm{~N}^{+}$calcd for $\mathrm{C}_{8} \mathrm{H}_{20} \mathrm{~N}, 130.15902$ found, 130.16005 .

\subsection{IR spectra of $1,1 a$ and $1 a 1$}

IR spectra of $\mathbf{1}, \mathbf{1} \mathbf{a} \cdot \mathbf{M e} \mathbf{e}_{\mathbf{4}} \mathbf{N}^{+}$and $\mathbf{1 a 1} \cdot \mathbf{M e} \mathbf{N}_{\mathbf{4}}^{+}$were recorded. When comparing the spectra of $\mathbf{1}$ and $\mathbf{1 a 1} \cdot \mathbf{M e}_{4} \mathbf{N}^{+}$it is seen that absorbance due to the $\mathrm{O}-\mathrm{H}$ stretch is missing in the spectrum of $1 \mathrm{a} 1 \cdot \mathbf{M e}_{4} \mathbf{N}^{+}$. There are two possible reasons, which may operate jointly: (1) The peak may be very wide. Wide peaks of similarly chelated protons have been observed before. $^{3}$ (2) The peak may be of low intensity due to a small change of the dipole moment of the system on vibration, because of the well delocalized charge in the anion. Also the position of the cation can be involved in reducing the dipole moment change. If the peak is wide and has low intensity then in may be hidden in the baseline. 


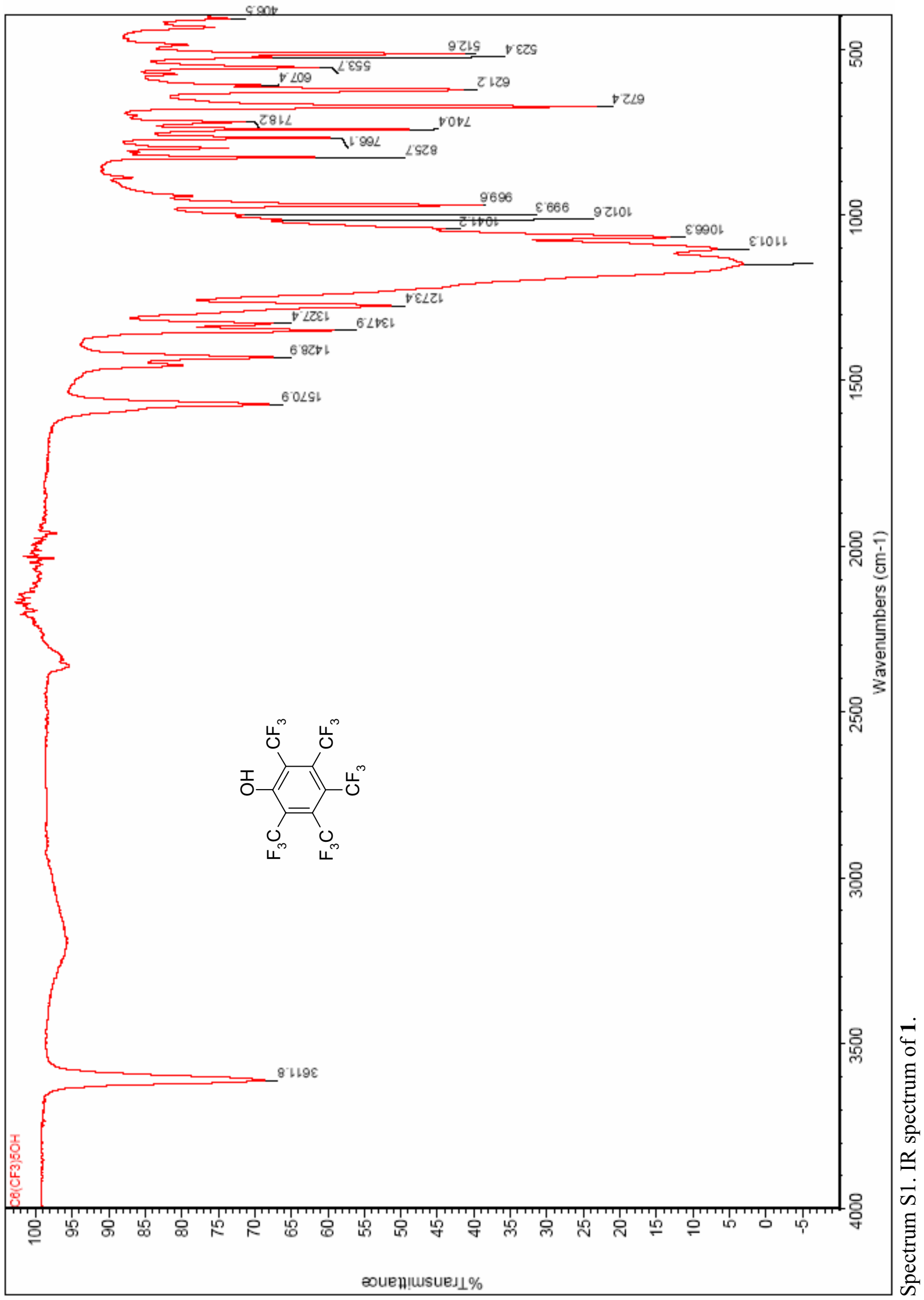




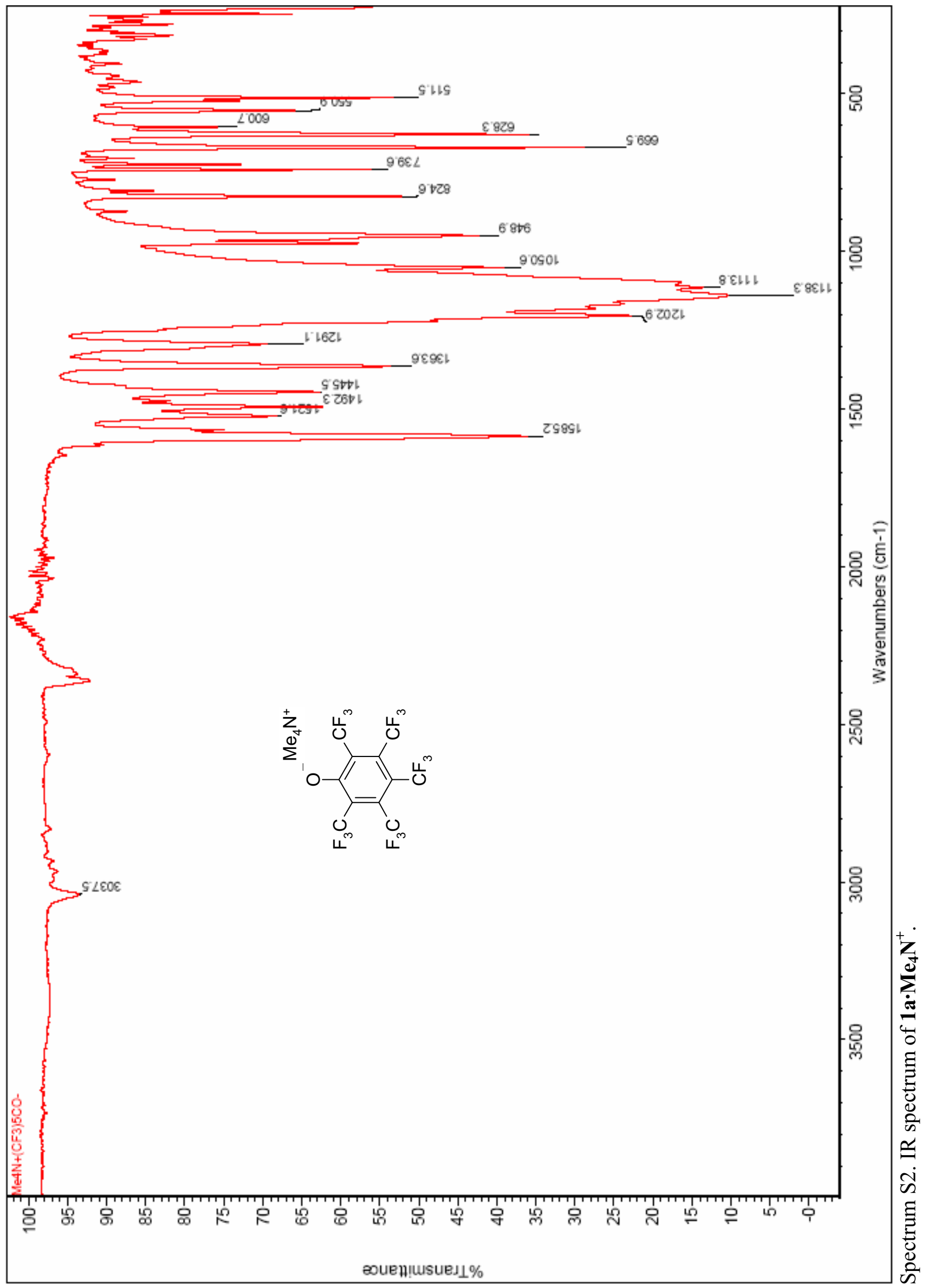




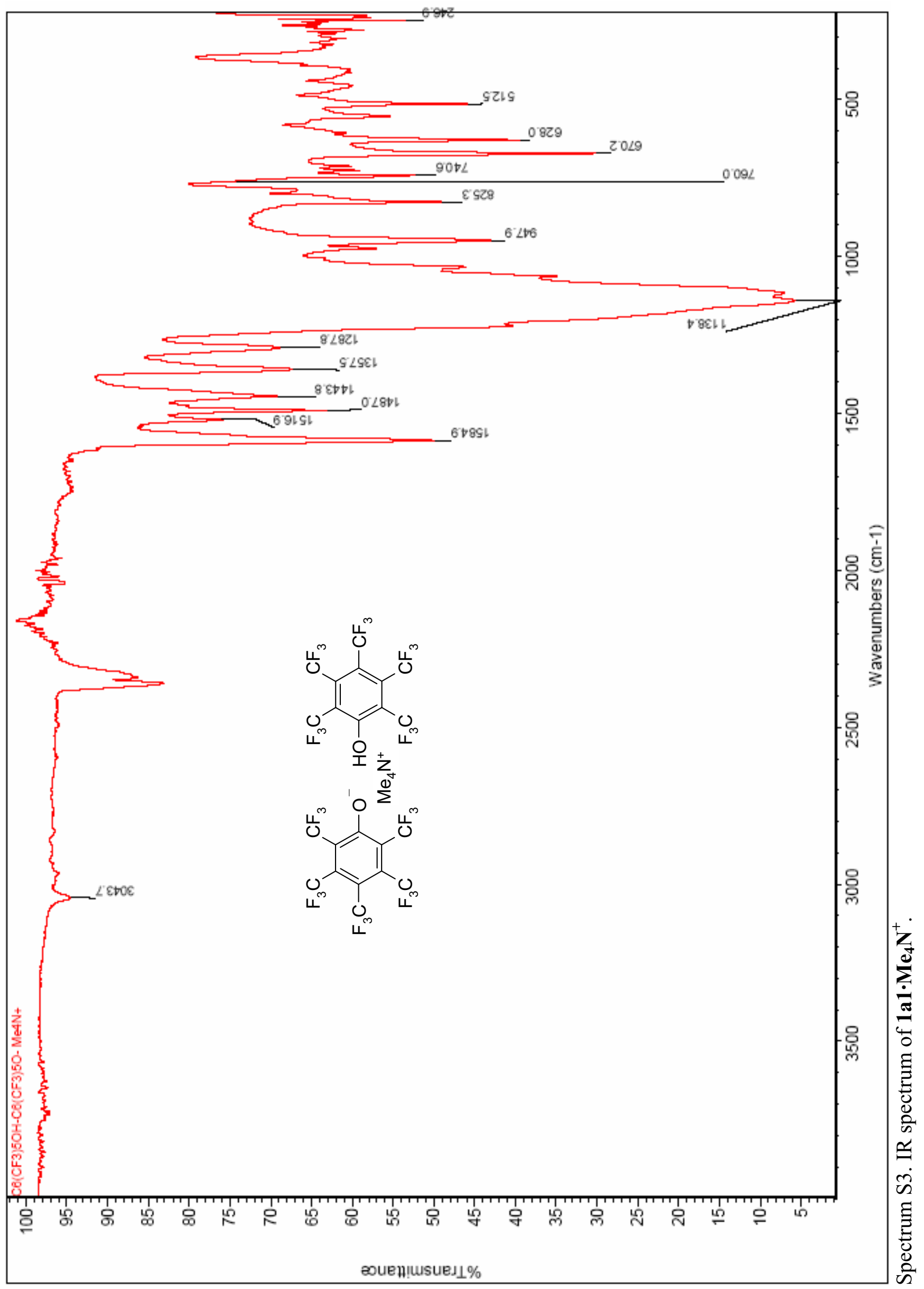


LL' $89 L^{-}$

EL'8SL-

ع9‘8L L-

カで๋レー

St'98-

†0'เ8-

Z9'08-

†乙'89-

89'ตะ-

$9 \varepsilon^{\prime} 乙 \varepsilon^{-}$

$08^{\prime} 8 \mathrm{C}^{-}$

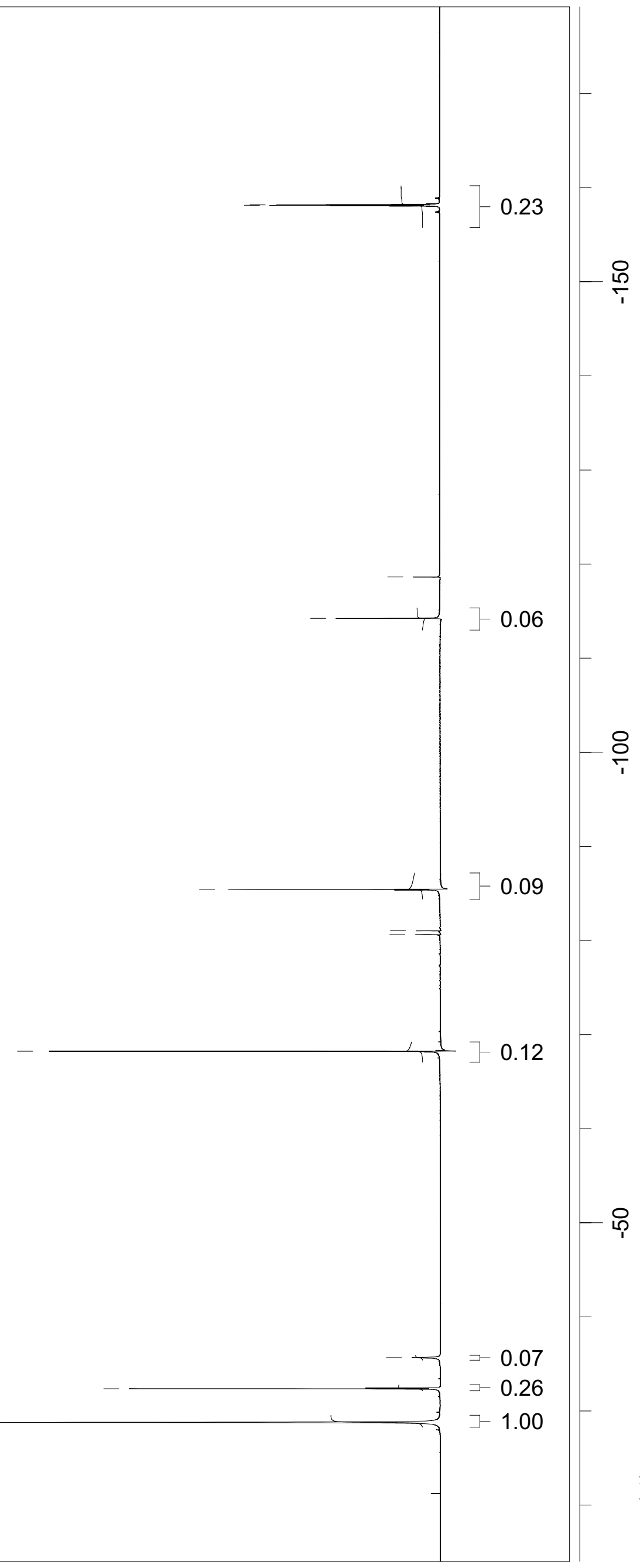

के

$\dddot{2}$
$\infty$
$\dot{0}$
$\dot{0}$
$\dot{0}$

$\infty$

i

$\therefore$

है के

2.

o

कี

㐫

오

业

官

$\infty$

ํㅗㅇ

.

政

$=$

舟

已.

焉

항

递

。ั

웅

焉

ధㅇ

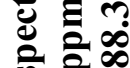

更

두

之े

$5 \ldots$

ซ๊

을

든

نㅇํㅇ

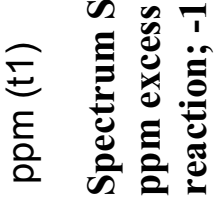


8L'89 L-

0t'18-

$86^{\prime} 08^{-}$

8L'乙ย'

$08^{\prime} 8 z^{-}$

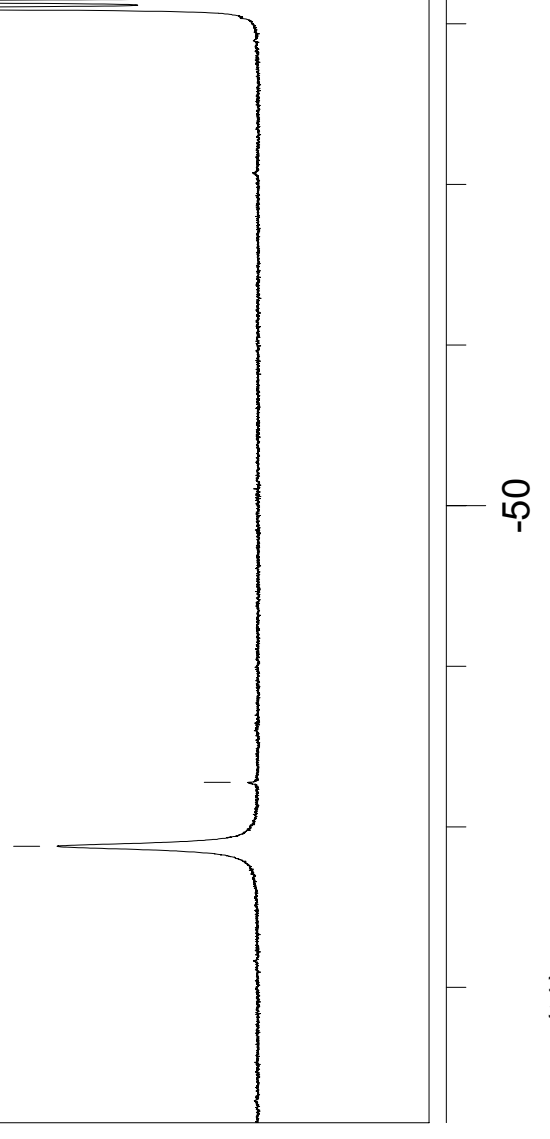

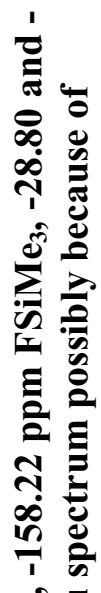

ज्ञ

음

ह

ํㅡㄴ 冚

ธู ฮ

$\infty$

를

ฮ

$\frac{0}{4}$

를

두

$\infty$

?

एँ

已

E

*

ฮ

क⿺辶万

우 芒

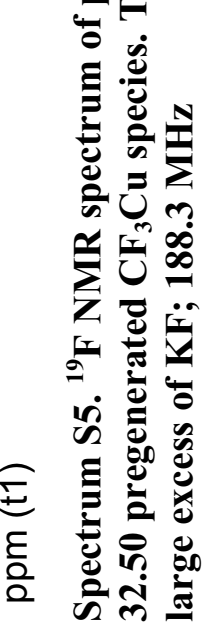


89L'L0GL $08-$

นงจ๋ย9-

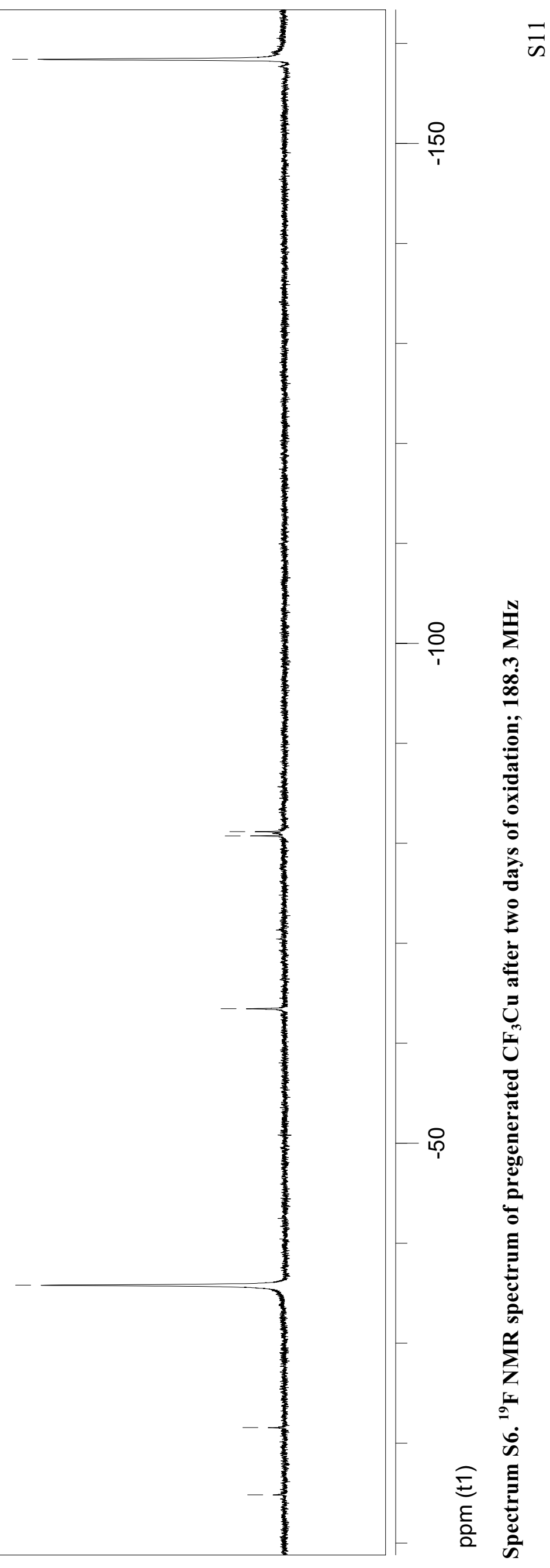

เ08 $98^{\circ}$

EGG'レー

6て8ナレー 


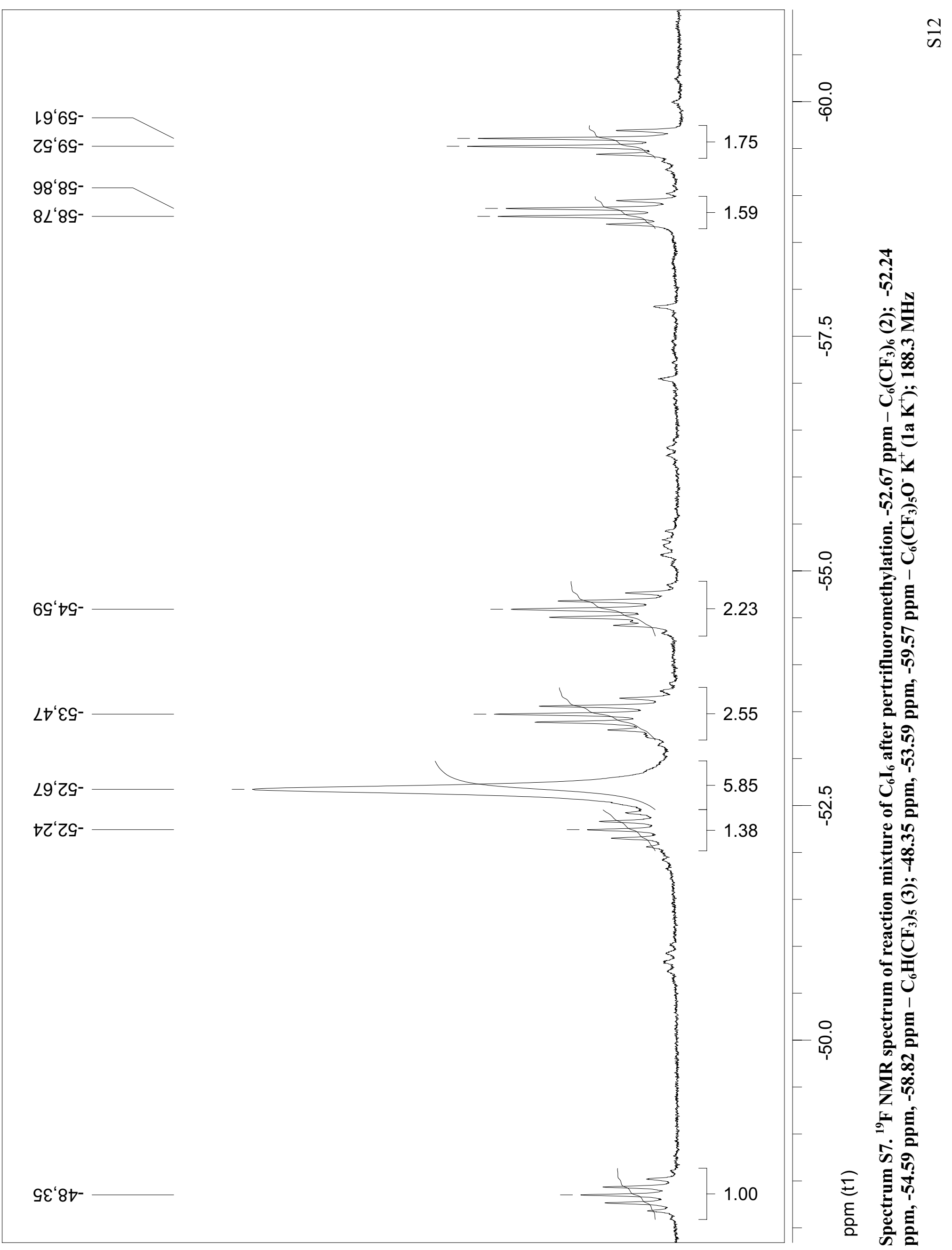




\section{Deprotonation of Pentakis(trifluoromethyl)benzene (3)}

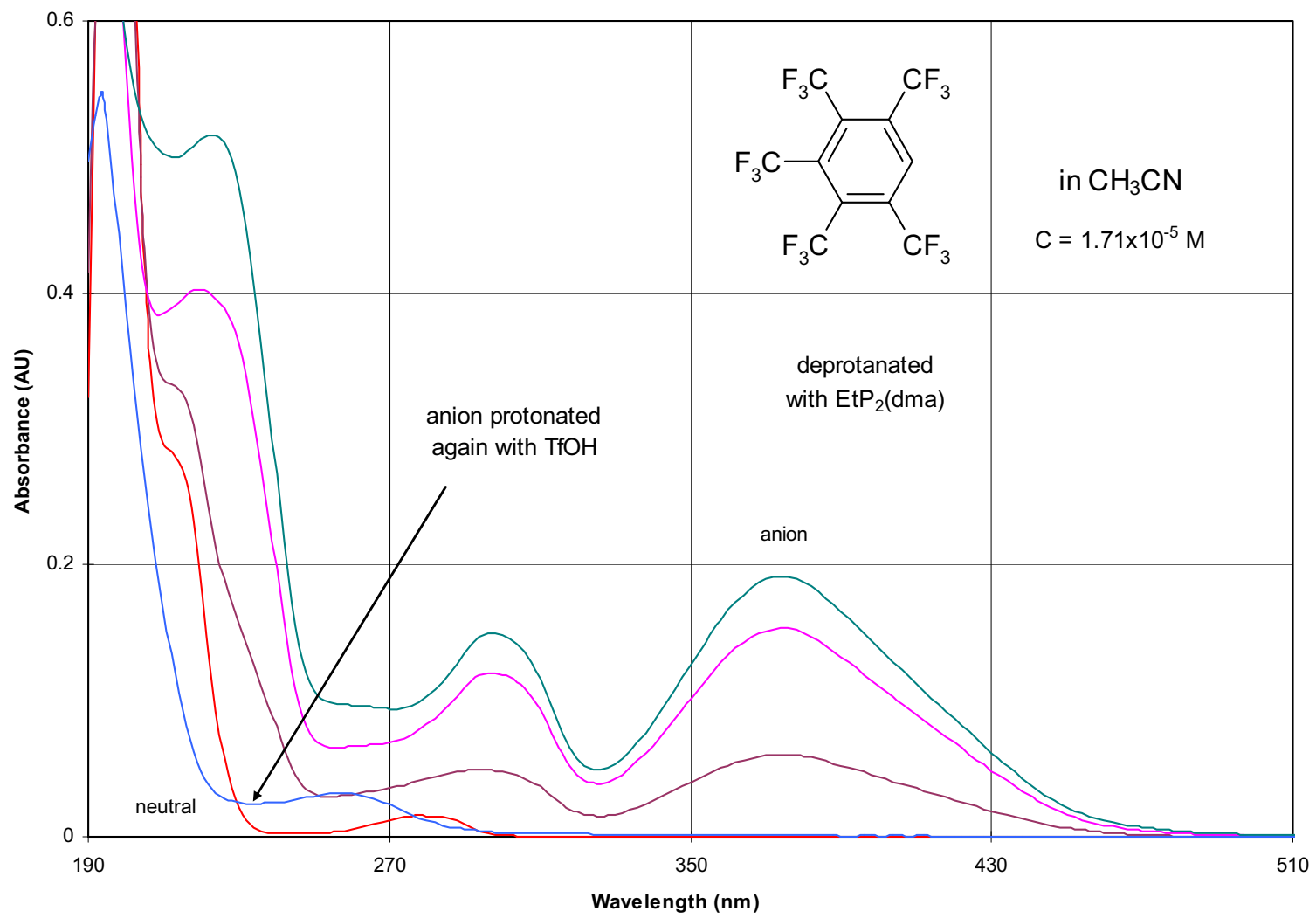

Figure S1. UV-vis spectra of titration of pentakis(trifluoromethyl)benzene (3) in acetonitrile (AN). For deprotonation the titration was carried out with phosphazene base $\mathrm{EtP}_{2}(\mathrm{dma})$. The deprotonated 3 (dark-green spectrum) was again protonated with TfOH. The obtained spectrum (blue) of neutral 3 differs slightly from the initial spectrum of 3 (red). The broad maximum at ca $250 \mathrm{~nm}$ on the final spectrum (blue) most probably comes from a decomposition product of $A N$ in the presence of strong $\operatorname{EtP}_{2}(d m a)$ base. 


\section{Determination of Acidities in Acetonitrile (AN) and in the Gas Phase}

\subsection{Detailed Description of Determination of $\Delta \mathrm{p} K_{a}$ Values in AN}

Reagents and Materials. Solution of trifluoromethanesulfonic acid (TfOH) (99+\%) or methanesulfonic acid ( $\geq 99 \%$ ) were used as acidic titrants and solutions of triethylamine (99\%), phosphazene bases $t$-BuP 1 (pyrr) $(\geq 98 \%)$ or $\mathrm{EtP}_{2}(\mathrm{dma})(>98 \%)$ were used as basic titrants.

Phenol ("pure") was sublimed, 3,5-bis(trifluoromethyl)phenol (95\%) was distilled under vacuum; 2-trifluoromethylphenol (97\%), 3-trifluoromethylphenol $\quad(99 \%), \quad 4-$ trifluoromethylphenol (97\%) were used as received.

Commercial AN with water concentration stated by producer below $0.005 \%$ (determined in our lab by coulometric Karl Fischer titration below 0.004\%) was used.

Calculation method based on spectra. If a neutral acid forms hydrogen-bonded complex (homoconjugate complex) with its anion extensively and if the protonation site is directly bound to an aromatic ring then abnormalities appear in UV-vis spectra during the titration of the pure compound. In particular the spectra do not have isosbestic points anymore evidencing that there is a more complex equilibrium in the solution than just between two forms of the compound. In this case, it is possible to calculate the spectra of the homoconjugate complex according to the concentration of the compound, amount of moles of basic titrant added and the homoconjugation constant $K_{\mathrm{AHA}}$.

Let us denote that the acid that forms a homoconjugate complex is $\mathrm{HA}_{1}$ and the acid that does not form a homoconjugate complex is acid $\mathrm{HA}_{2}$. The two acids do not form a heteroconjugate complex.

$K_{\mathrm{AHA}}$ (the homoconjugation constant) is the constant of formation of the homoconjugate complex $\mathrm{A}_{1}{ }^{-} \cdots \mathrm{HA}_{1}$ :

$$
K_{\mathrm{AHA}}=\frac{a\left(\mathrm{~A}_{1}^{-} \cdots \mathrm{HA}_{1}\right)}{a\left(\mathrm{~A}_{1}^{-}\right) \cdot a\left(\mathrm{HA}_{1}\right)}
$$


We assume that the activities in equation 1 can be replaced with concentrations and for now on we use only concentrations instead of activities:

$$
K_{\mathrm{AHA}}=\frac{\left[\mathrm{A}_{1}^{-} \cdots \mathrm{HA}_{1}\right]}{\left[\mathrm{A}_{1}^{-}\right] \cdot\left[\mathrm{HA}_{1}\right]}
$$

At a given point of the titration the concentrations of the neutral $\mathrm{HA}_{1}$ and anion $\mathrm{A}_{1}{ }^{-}$in solution, which we can calculate directly from the mass of added titrant to the solution are apparent concentrations, because a part of the species are bound into the homoconjugate complex:

$$
\left[\mathrm{HA}_{1}\right]_{\mathrm{app}}=\left[\mathrm{HA}_{1}\right]+\left[\mathrm{A}_{1}^{-} \cdots \mathrm{HA}_{1}\right] \text { and }\left[\mathrm{A}_{1}^{-}\right]_{\mathrm{app}}=\left[\mathrm{A}_{1}^{-}\right]+\left[\mathrm{A}_{1}^{-} \cdots \mathrm{HA}_{1}\right]
$$

The same apparent concentrations can be found via the analytical concentration $C_{1}$ of $\mathrm{HA}_{1}$ and its apparent ionization level $\alpha_{1 \mathrm{app}}$ :

$$
\left[\mathrm{HA}_{1}\right]_{\mathrm{app}}=\left(1-\alpha_{1 \mathrm{app}}\right) \cdot C_{1} \text { and }\left[\mathrm{A}_{1}^{-}\right]_{\mathrm{app}}=\alpha_{1 \mathrm{app}} \cdot C_{1}
$$

The apparent ionization level $\alpha_{1 \text { app }}$ is the ratio of mass of titrant added up to the current point and the total mass of titrant added to the solution by the end of the titration. The apparent ionization level is the ionization level, which contains also the concentration of the homoconjugate complex.

Using equations 2 and 3 we get the quadratic equation:

$$
\left[\mathrm{A}_{1}^{-} \cdots \mathrm{HA}_{1}\right]^{2}+\left(-\left[\mathrm{A}_{1}^{-}\right]_{\mathrm{app}}-\left[\mathrm{HA}_{1}\right]_{\mathrm{app}}-1 / K_{\mathrm{aha}}\right) \cdot\left[\mathrm{A}_{1}^{-} \cdots \mathrm{HA}_{1}\right]+\left[\mathrm{A}_{1}^{-}\right]_{\mathrm{app}} \cdot\left[\mathrm{HA}_{1}\right]_{\mathrm{app}}=0
$$

The concentration of the complex $\mathrm{A}_{1}{ }^{-} \cdots \mathrm{HA}_{1}$ can be found:

$$
\begin{aligned}
& {\left[\mathrm{A}_{1}^{-} \cdots \mathrm{HA}_{1}\right]=} \\
& =\frac{\frac{1}{K_{\text {aha }}}+\left[\mathrm{HA}_{1}\right]_{\mathrm{app}}+\left[\mathrm{A}_{1}^{-}\right]_{\mathrm{app}}+\sqrt{\left(-\left[\mathrm{A}_{1}^{-}\right]_{\mathrm{app}}-\left[\mathrm{HA}_{1}\right]_{\mathrm{app}}-\frac{1}{K_{\text {aha }}}\right)^{2}-4 \cdot\left[\mathrm{A}_{1}^{-}\right]_{\mathrm{app}} \cdot\left[\mathrm{HA}_{1}\right]_{\mathrm{app}}}}{2}
\end{aligned}
$$

After we know $\left[\mathrm{A}_{1}^{-} \cdots \mathrm{HA}_{1}\right]$, from equations 3 it is possible to calculate the real concentrations of $\left[\mathrm{HA}_{1}\right]$ and $\left[\mathrm{A}_{1}^{-}\right]$. Using the analytical concentration $C_{1}$ of the acid $\mathrm{HA}_{1}$ it is possible to get the relative concentrations of the species $\mathrm{HA}_{1}, \mathrm{~A}_{1}{ }^{-}$and $\mathrm{A}_{1}{ }^{-} \cdots \mathrm{HA}_{1}$ in the solutions with different ionization levels (corresponding to different points of the titration). The relative concentrations $\left[\mathrm{HA}_{1}\right]_{\mathrm{rel}},\left[\mathrm{A}_{1}{ }^{-}\right]_{\mathrm{rel}}$ and $\left[\mathrm{A}_{1}{ }^{-} \cdots \mathrm{HA}_{1}\right]_{\mathrm{rel}}$ show how large is the proportion of the corresponding species in the solution. 
From these relative concentrations it is now possible to calculate the spectrum of the homoconjugate complex. The spectrum of the same species has to be the same in solutions of different ionization levels. The total absorbance of the solution in different protonation level using relative concentration of particles at wavelength $\lambda$ is:

$$
A_{1 \text { pure }}^{\lambda}=\left[\mathrm{A}_{1}^{-}\right]_{\text {rel }} A_{\mathrm{A}_{1}^{-} \text {pure }}^{\lambda}+\left[\mathrm{HA}_{1}\right]_{\text {rel }} A_{\mathrm{HA}_{\text {p pure }}}^{\lambda}+\left[\mathrm{A}_{1}^{-} \cdots \mathrm{HA}_{1}\right]_{\mathrm{rel}} A_{\mathrm{A}_{1} \cdots \mathrm{HA}_{1}}^{\lambda}
$$

The $A_{1}{ }^{\lambda}$ values are the spectra of the respective individual species at concentration $C_{1}$. From the equation 7 we get the absorbance $A_{\mathrm{A}_{1} \cdots \mathrm{HA}_{1}}^{\lambda}$ of the hypothetical solution containing only the homoconjugate complex $\mathrm{A}_{1}^{-} \cdots \mathrm{HA}_{1}$ at concentration $C_{1}$ :

$$
A_{\mathrm{A}_{1} \cdots \mathrm{HA}_{1}}^{\lambda}=\frac{A_{\text {lpure }}^{\lambda}-\left[\mathrm{A}_{1}^{-}\right]_{\mathrm{rel}} A_{\mathrm{A}_{1} \text { pure }}^{\lambda}-\left[\mathrm{HA}_{1}\right]_{\mathrm{rel}} A_{\mathrm{HA}_{1} \text { pure }}^{\lambda}}{\left[\mathrm{A}_{1}^{-} \cdots \mathrm{HA}_{1}\right]_{\mathrm{rel}}}
$$

The spectra of the homoconjugate complexes were calculated from different titration points in the middle of the titration run (where the concentration of the homoconjugate is the highest) and were averaged. The averaged spectrum is used for calculation of the $\Delta \mathrm{p} K_{\mathrm{a}}$ value as described below.

To calculate the $\Delta \mathrm{p} K_{\mathrm{a}}$ value from a titration point of the mixture it is first necessary to find the coefficients $c_{1}$ and $c_{2}$, which show the ratios of concentrations in the titrated mixture of two acids $\mathrm{HA}_{1}$ and $\mathrm{HA}_{2}$ and in the separate titrations of the pure acids. The calculation of these two coefficients is described in ref 7 .

The absorbance of the solution containing mixture of the two acids at wavelength $\lambda$ can be calculated as follows:

$$
A^{\lambda}=A_{1}^{\lambda}+A_{2}^{\lambda}
$$

Where $A_{1}^{\lambda}$ and $A_{2}^{\lambda}$ are the absorbances of $\mathrm{HA}_{1}$ and $\mathrm{HA}_{2}$ in mixture. $A_{1}^{\lambda}$ can be expressed as the sum of absorbances of the pure forms multiplied by their relative concentrations in the mixture:

$$
A_{1}^{\lambda}=c_{1}\left(\left[\mathrm{~A}_{1}^{-}\right]_{\text {rel,mix }} A_{\mathrm{A}_{1}^{-} \text {pure }}^{\lambda}+\left[\mathrm{HA}_{1}\right]_{\text {rel,mix }} A_{\mathrm{HA}_{1} \text { pure }}^{\lambda}+\left[\mathrm{A}_{1}^{-} \cdots \mathrm{HA}_{1}\right]_{\text {rel,mix }} A_{\mathrm{A}_{1} \cdots \mathrm{HA}_{1}}^{\lambda}\right)
$$

The absorbance of acid 2 at certain ionization level can be also written using absorbances of pure forms of acid 2 :

$$
A_{2}^{\lambda}=c_{2}\left(\alpha_{2} A_{\mathrm{A}_{2}^{-} \text {pure }}^{\lambda}+\left(1-\alpha_{2}\right) A_{\mathrm{HA}_{2} \text { pure }}^{\lambda}\right)
$$


Ionization level of acid 2 is found at a longer wavelength where the acid 1 does not absorb:

$$
\alpha_{2}=\frac{A_{x}^{\lambda}}{A_{\mathrm{A}_{2}}^{\lambda}}
$$

It was in all cases possible to find reference acids $\mathrm{HA}_{2}$ that had absorption bands at longer wavelengths than the phenols.

The apparent concentrations of $\mathrm{HA}_{1}$ and $\mathrm{A}_{1}{ }^{-}$in the mixture are found from the apparent ionization level of $\mathrm{HA}_{1}$ in the mixture $\alpha_{1 \text { app,mix }}$ as follows:

$$
\left[\mathrm{HA}_{1}\right]_{\mathrm{app}, \text { mix }}=\left(1-\alpha_{1 \text { app,mix }}\right) \cdot c_{1} \cdot C_{1} \quad \text { and } \quad\left[\mathrm{A}_{1}^{-}\right]_{\mathrm{app}, \text { mix }}=\alpha_{1 \text { app,mix }} \cdot c_{1} \cdot C_{1}
$$

The concentration of the homoconjugate complex $\left[\mathrm{A}_{1}{ }^{-\cdots} \mathrm{HA}_{1}\right]_{\text {mix }}$ is found as follows:

$$
\begin{aligned}
& {\left[\mathrm{A}_{1}^{-} \cdots \mathrm{HA}_{1}\right]_{\text {mix }}=} \\
& =\frac{\frac{1}{K_{\text {aha }}}+\left[\mathrm{HA}_{1}\right]_{\mathrm{app}, \text { mix }}+\left[\mathrm{A}_{1}^{-}\right]_{\mathrm{app}, \text { mix }}+\sqrt{\left(-\left[\mathrm{A}_{1}^{-}\right]_{\mathrm{app}, \text { mix }}-\left[\mathrm{HA}_{1}\right]_{\mathrm{app}, \text { mix }}-\frac{1}{K_{\text {aha }}}\right)^{2}-4 \cdot\left[\mathrm{A}_{1}^{-}\right]_{\mathrm{app}, \text { mix }} \cdot\left[\mathrm{HA}_{1}\right]_{\mathrm{app}, \text { mix }}}}{2}
\end{aligned}
$$

The real equilibrium concentrations of $\mathrm{HA}_{1}$ and $\mathrm{A}_{1}{ }^{-}$in the mixture are found as follows:

$$
\begin{aligned}
& {\left[\mathrm{HA}_{1}\right]_{\text {mix }}=\left[\mathrm{HA}_{1}\right]_{\mathrm{app}, \text { mix }}-\left[\mathrm{A}_{1}^{-} \cdots \mathrm{HA}_{1}\right]_{\text {mix }}} \\
& {\left[\mathrm{A}_{1}^{-}\right]_{\text {mix }}=\left[\mathrm{A}_{1}^{-}\right]_{\mathrm{app}, \text { mix }}-\left[\mathrm{A}_{1}^{-}{ }^{-} \mathrm{HA}_{1}\right]_{\text {mix }}}
\end{aligned}
$$

The relative concentrations $\left[\mathrm{HA}_{1}\right]_{\mathrm{rel}, \mathrm{mix}},\left[\mathrm{A}_{1}^{-}\right]_{\mathrm{rel}, \mathrm{mix}}$ and $\left[\mathrm{A}_{1}{ }^{-} \cdots \mathrm{HA}_{1}\right]_{\mathrm{rel}, \mathrm{mix}}$ can be found from the respective absolute concentrations:

$$
\left[\mathrm{HA}_{1}\right]_{\mathrm{rel}, \text { mix }}=\frac{\left[\mathrm{HA}_{1}\right]_{\text {mix }}}{C_{1}} \text { and }\left[\mathrm{A}_{1}^{-}\right]_{\mathrm{rel}, \text { mix }}=\frac{\left[\mathrm{A}_{1}^{-}\right]_{\text {mix }}}{C_{1}}
$$

After combining equations 9, 10 and 11, we get:

$$
\begin{aligned}
& A^{\lambda}=c_{1}\left(\left[\mathrm{~A}_{1}^{-}\right]_{\text {rel,mix }} A_{\mathrm{A}_{1}^{-} \text {pure }}^{\lambda}+\left[\mathrm{HA}_{1}\right]_{\mathrm{rel}, \text { mix }} A_{\mathrm{HA}_{1} \text { pure }}^{\lambda}+\left[\mathrm{A}_{1}^{-} \cdots \mathrm{HA}_{1}\right]_{\mathrm{rel}, \text { mix }} A_{\mathrm{A}_{1} \cdots \mathrm{HA}_{1}}^{\lambda}\right)+ \\
& +c_{2}\left(\alpha_{2} A_{\mathrm{A}_{2} \text { pure }}^{\lambda}+\left(1-\alpha_{2}\right) A_{\mathrm{HA}_{2} \text { pure }}^{\lambda}\right)
\end{aligned}
$$

Equation 18 models the spectrum of the mixture of acids $\mathrm{HA}_{1}$ and $\mathrm{HA}_{2}$ via the spectra of the pure forms of the acids and the concentrations of the species in the mixture. Least 
squares minimization procedure between two different spectra - experimental and modeled spectra (see equations 19 and 20) can be used.

$$
S^{2}=\sum_{\lambda}\left(A_{\mathrm{exp}}^{\lambda}-A^{\lambda}\right)^{2}
$$

This equation can be rewritten as:

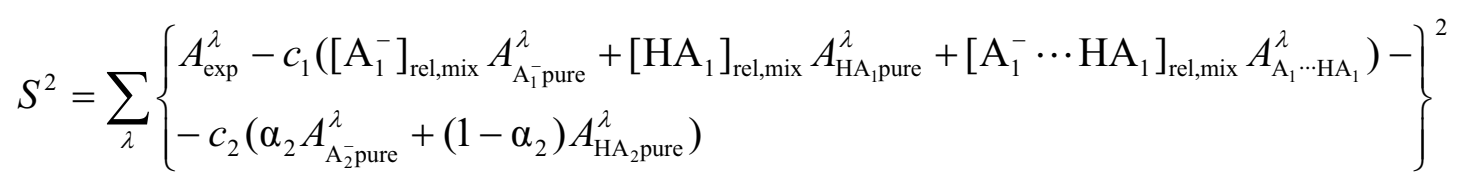

The deprotonation level $\alpha_{2}$ of $\mathrm{HA}_{2}$ and the apparent deprotonation level $\alpha_{1 \text { app }}$ of $\mathrm{HA}_{1}$ corresponding to the minimum difference between the experimental spectra and those calculated by eq 18 are found by least squares minimization of $S^{2}$ over the used wavelength range using the two $\alpha$ values as adjustable parameters. The concentrations $\left[\mathrm{HA}_{1}\right]_{\mathrm{rel}, \mathrm{mix}},\left[\mathrm{A}_{1}^{-}\right]_{\mathrm{rel}, \mathrm{mix}}$ and $\left[\mathrm{A}_{1}^{-} \cdots \mathrm{HA}_{1}\right]_{\mathrm{rel}, \mathrm{mix}}$ are linked to $\alpha_{1 \text { app }}$ by equations 13 to 17 . The real protonation level $\alpha_{1}$ is found as follows:

$$
\alpha_{1}=\frac{\left[\mathrm{A}_{1}^{-}\right]_{\text {mix }}}{\left[\mathrm{A}_{1}^{-}\right]_{\text {mix }}+\left[\mathrm{HA}_{1}\right]_{\text {mix }}}
$$

In the case of phenols, the minimization procedures were usually carried out in the wavelength range $250-290 \mathrm{~nm}$. In this range the difference of the spectra of the corresponding phenol and phenoxide anion is the largest.

Using the $\alpha_{1}$ and $\alpha_{2}$ values, the $\Delta \mathrm{p} K_{\mathrm{a}}$ at the given titration point can be expressed as:

$$
\Delta \mathrm{p} K_{a}=\log \frac{\alpha_{1}\left(1-\alpha_{2}\right)}{\left(1-\alpha_{1}\right) \alpha_{2}}
$$

$\Delta \mathrm{p} K_{\mathrm{a}}$ values are found from every titration point during the titration of mixture where protonation levels are usually between $5 \%$ to $95 \%$ for both acids. 
Table S1. Results of $\Delta \mathrm{p} K_{\mathrm{a}}$ measurements in AN.

\begin{tabular}{|c|c|c|c|c|c|}
\hline Acid (A) & Reference acid (Ra) & $\mathrm{p} K_{\mathrm{a}}(\mathrm{Ra})$ & $\Delta \mathrm{p} K_{\mathrm{a}}^{a}$ & $\mathrm{p} K_{\mathrm{a}}(\mathrm{A})$ & $\begin{array}{l}\text { Average } \\
\mathbf{p} \boldsymbol{K}_{\mathbf{a}}(\mathrm{A})\end{array}$ \\
\hline \multirow[t]{2}{*}{ Phenol } & 9- $\mathrm{C}_{6} \mathrm{~F}_{5}$-Fluorene & 28.12 & -1.06 & 29.18 & 29.14 \\
\hline & $\left(4-\mathrm{Me}-\mathrm{C}_{6} \mathrm{~F}_{4}\right)\left(\mathrm{C}_{6} \mathrm{H}_{5}\right) \mathrm{CHCN}$ & 26.97 & -2.12 & 29.09 & \\
\hline \multirow[t]{4}{*}{ 3-CF ${ }_{3}-$ Phenol } & $\left(4 \mathrm{NC}_{5} \mathrm{~F}_{4}\right)\left(\mathrm{C}_{6} \mathrm{~F}_{5}\right) \mathrm{NH}$ & 26.34 & -0.40 & 26.74 & 26.50 \\
\hline & $\left(4-\mathrm{Me}-\mathrm{C}_{6} \mathrm{~F}_{4}\right)\left(\mathrm{C}_{6} \mathrm{H}_{5}\right) \mathrm{CHCN}$ & 26.97 & 0.63 & 26.34 & \\
\hline & $\left(\mathrm{C}_{6} \mathrm{~F}_{5}\right)\left(\mathrm{C}_{6} \mathrm{H}_{5}\right) \mathrm{CHCN}$ & 26.15 & -0.20 & 26.35 & \\
\hline & $\left(4-\mathrm{Me}_{2} \mathrm{~N}-\mathrm{C}_{6} \mathrm{~F}_{4}\right)\left(\mathrm{C}_{6} \mathrm{~F}_{5}\right) \mathrm{NH}$ & 25.12 & -1.46 & 26.58 & \\
\hline \multirow[t]{2}{*}{ 4-CF ${ }_{3}-$ Phenol } & $\left(\mathrm{C}_{6} \mathrm{~F}_{5}\right)\left(\mathrm{C}_{6} \mathrm{H}_{5}\right) \mathrm{CHCN}$ & 26.15 & 0.60 & 25.55 & 25.54 \\
\hline & $\left(4-\mathrm{Me}-\mathrm{C}_{6} \mathrm{~F}_{4}\right)\left(\mathrm{C}_{6} \mathrm{~F}_{5}\right) \mathrm{NH}$ & 24.95 & -0.57 & 25.52 & \\
\hline \multirow[t]{2}{*}{ 2-CF -Phenol } & $\left(\mathrm{C}_{6} \mathrm{~F}_{5}\right)\left(\mathrm{C}_{6} \mathrm{H}_{5}\right) \mathrm{CHCN}$ & 26.15 & 1.35 & 24.80 & 24.88 \\
\hline & $\left(4-\mathrm{Me}-\mathrm{C}_{6} \mathrm{~F}_{4}\right)\left(\mathrm{C}_{6} \mathrm{~F}_{5}\right) \mathrm{NH}$ & 24.95 & -0.01 & 24.96 & \\
\hline \multirow[t]{2}{*}{$3,5-\left(\mathrm{CF}_{3}\right)_{2}$-Phenol } & Fluoradene & 23.90 & 0.18 & 23.72 & 23.78 \\
\hline & 2- $\mathrm{NO}_{2}$-Phenol & 22.87 & -0.97 & 23.84 & \\
\hline \multirow[t]{3}{*}{$\mathrm{C}_{6}\left(\mathrm{CF}_{3}\right)_{5} \mathrm{OH}, 1$} & Picric acid & 11.00 & 0.53 & 10.47 & 10.46 \\
\hline & $4-\mathrm{NO}_{2}-\mathrm{C}_{6} \mathrm{H}_{4}-\mathrm{CH}(\mathrm{CN})_{2}$ & 11.61 & 1.15 & 10.46 & \\
\hline & $4-\mathrm{CF}_{3}-\mathrm{C}_{6} \mathrm{~F}_{4}-\mathrm{CH}(\mathrm{CN})_{2}$ & 10.91 & -0.27 & 10.46 & \\
\hline \multirow[t]{4}{*}{$\mathrm{C}_{6}\left(\mathrm{CF}_{3}\right)_{5} \mathrm{NH}_{2}, 5$} & 2- $\mathrm{NO}_{2}$-Phenol & 22.85 & -1.70 & 24.55 & 24.57 \\
\hline & Octafluorofluorene & 24.49 & -0.10 & 24.59 & \\
\hline & Fluoradene & 23.90 & -0.72 & 24.62 & \\
\hline & $\left(4-\mathrm{Me}-\mathrm{C}_{6} \mathrm{~F}_{4}\right)_{2} \mathrm{CHCN}$ & 22.80 & -1.73 & 24.53 & \\
\hline \multirow[t]{2}{*}{$\mathrm{C}_{6}\left(\mathrm{CF}_{3}\right)_{5} \mathrm{CH}_{3}, 6$} & 9- $\mathrm{C}_{6} \mathrm{~F}_{5}$-Fluorene & 28.11 & -0.80 & 28.9 & $28.7^{b}$ \\
\hline & $\left(\mathrm{NC}_{5} \mathrm{~F}_{4}\right)\left(4-\mathrm{Me}-\mathrm{C}_{6} \mathrm{H}_{4}\right) \mathrm{NH}$ & 26.84 & -1.74 & 28.6 & \\
\hline \multirow[t]{2}{*}{$\mathrm{C}_{6}\left(\mathrm{CF}_{3}\right)_{5} \mathrm{CH}(\mathrm{CN})_{2}, 7$} & $4-\mathrm{NO}_{2}-\mathrm{C}_{6} \mathrm{H}_{4} \mathrm{SO}_{2} \mathrm{NHSO}_{2} \mathrm{C}_{6} \mathrm{H}_{4}-4-\mathrm{Cl}$ & $9.16^{c}$ & 0.32 & 8.84 & 8.86 \\
\hline & $\left(4-\mathrm{NO}_{2}-\mathrm{C}_{6} \mathrm{H}_{4}-\mathrm{SO}_{2}\right)_{2} \mathrm{NH}$ & $8.18^{c}$ & -0.69 & 8.87 & \\
\hline \multirow[t]{2}{*}{$2,3,4,6-\left(\mathrm{CF}_{3}\right)_{4}-\mathrm{C}_{6} \mathrm{HCH}(\mathrm{CN})_{2}$} & $4-\mathrm{NO}_{2}-\mathrm{C}_{6} \mathrm{H}_{4} \mathrm{SO}_{2} \mathrm{NHSO}_{2} \mathrm{C}_{6} \mathrm{H}_{4}-4-\mathrm{Cl}$ & $9.16^{c}$ & -1.30 & 10.46 & 10.45 \\
\hline & $4-\mathrm{CF}_{3}-\mathrm{C}_{6} \mathrm{~F}_{4} \mathrm{CH}(\mathrm{CN})_{2}$ & 10.19 & -0.24 & 10.43 & \\
\hline
\end{tabular}

${ }^{a} \mathrm{p} K_{\mathrm{a}}(\mathrm{Ra})-\mathrm{p} K_{\mathrm{a}}(\mathrm{A}){ }^{b}$ Approximate value $\left( \pm 0.5 \mathrm{p} K_{\mathrm{a}} \text { units }\right)^{c}$ Revised values 


\subsection{Results of Gas-phase Acidity Measurements}

Table S2. Results of the experimental $\Delta G_{\text {acid }}$ measurements. ${ }^{a}$

\begin{tabular}{|l|l|c|c|}
\hline $\mathbf{A}_{\mathbf{i}} \mathbf{H}$ & $\mathbf{A}_{\mathbf{0}} \mathbf{H}^{\boldsymbol{b}}$ & $\Delta \boldsymbol{G}_{\text {acid }}\left(\mathbf{A}_{\mathbf{0}} \mathbf{H}\right)^{c}$ & $\delta \Delta \boldsymbol{G}_{\text {acid }}$ \\
\hline $\mathrm{C}_{6}\left(\mathrm{CF}_{3}\right)_{5} \mathrm{H} \mathrm{(3)}$ & $\left(\mathrm{C}_{6} \mathrm{~F}_{5}\right)_{2} \mathrm{PhCH}$ & 328.4 & 2.7 \\
\hline $\mathrm{C}_{6}\left(\mathrm{CF}_{3}\right)_{5} \mathrm{CH}_{3}(\mathbf{6})$ & $\mathrm{C}_{6} \mathrm{~F}_{5} \mathrm{OH}$ & 320.8 & -1.5 \\
\hline $\mathrm{C}_{6}\left(\mathrm{CF}_{3}\right)_{5} \mathrm{CH}_{3}(\mathbf{6})$ & $\left(\mathrm{C}_{6} \mathrm{~F}_{5}\right)_{2} \mathrm{NH}$ & 316.4 & 2.0 \\
\hline $\mathrm{C}_{6}\left(\mathrm{CF}_{3}\right)_{5} \mathrm{NH}_{2}(\mathbf{5})$ & $\mathrm{C}_{6} \mathrm{H}_{5} \mathrm{NHSO}_{2} \mathrm{CF}_{3}$ & 313.5 & -3.2 \\
\hline $\mathrm{C}_{6}\left(\mathrm{CF}_{3}\right)_{5} \mathrm{NH}_{2}(\mathbf{5})$ & $\left(\mathrm{CF}_{3} \mathrm{CO}\right)_{2} \mathrm{NH}$ & 307.5 & 3.7 \\
\hline $\mathrm{C}_{6}\left(\mathrm{CF}_{3}\right)_{5} \mathrm{OH}(\mathbf{1})$ & $\left(\mathrm{CF}_{3} \mathrm{SO}_{2}\right)_{2} \mathrm{NH}$ & 291.8 & 4.1 \\
\hline $\mathrm{C}_{6}\left(\mathrm{CF}_{3}\right)_{5} \mathrm{OH}(\mathbf{1})$ & $\mathrm{CH}_{3} \mathrm{C}_{6} \mathrm{H}_{4} \mathrm{~S}(=\mathrm{NTf})_{2} \mathrm{NH}_{2}$ & $299.7^{d}$ & 0.4 \\
\hline $\mathrm{C}_{6}\left(\mathrm{CF}_{3}\right)_{5} \mathrm{OH}(\mathbf{1})$ & $\mathrm{C}_{6} \mathrm{H}_{5} \mathrm{CHTf}_{2}$ & 301.3 & -0.7 \\
\hline $\mathrm{C}_{6}\left(\mathrm{CF}_{3}\right)_{5} \mathrm{OH}(\mathbf{1})$ & $\mathrm{Tf}_{2} \mathrm{CH}_{2}$ & 301.5 & -1.7 \\
\hline $\mathrm{C}_{6}\left(\mathrm{CF}_{3}\right)_{5} \mathrm{OH}(\mathbf{1})$ & $\left(\mathrm{NC}_{5} \mathrm{C}_{6} \mathrm{CH}_{3}\right.$ & $302.1^{e}$ & -2.3 \\
\hline
\end{tabular}

${ }^{a} \delta \Delta G_{\text {acid }}=\Delta G_{\text {acid }}\left(\mathrm{A}_{\mathrm{i}} \mathrm{H}\right)-\Delta G_{\text {acid }}\left(\mathrm{A}_{0} \mathrm{H}\right)$. All values are given in $\mathrm{kcal} \cdot \mathrm{mol}^{-1}(1 \mathrm{cal}=4.184 \mathrm{~J})$.

${ }^{b}$ Tf denotes $\mathrm{CF}_{3} \mathrm{SO}_{2} .{ }^{c} \mathrm{GA}\left(\mathrm{A}_{0} \mathrm{H}\right)$ values taken from ref 8 , if not indicated otherwise. ${ }^{d}$ Ref 9. ${ }^{e} \operatorname{Ref} 10$. 


\subsection{Acidities of Pentakis(trifluoromethyl)phenyl Derivatives in Gas Phase, Correlations: Experimental $\Delta \boldsymbol{G}_{\text {acid }}$ vs. Computational $\Delta \boldsymbol{G}_{\text {acid }}$}

Table S3. Phenols, OH Acids, Carbocyclic acids, and others.

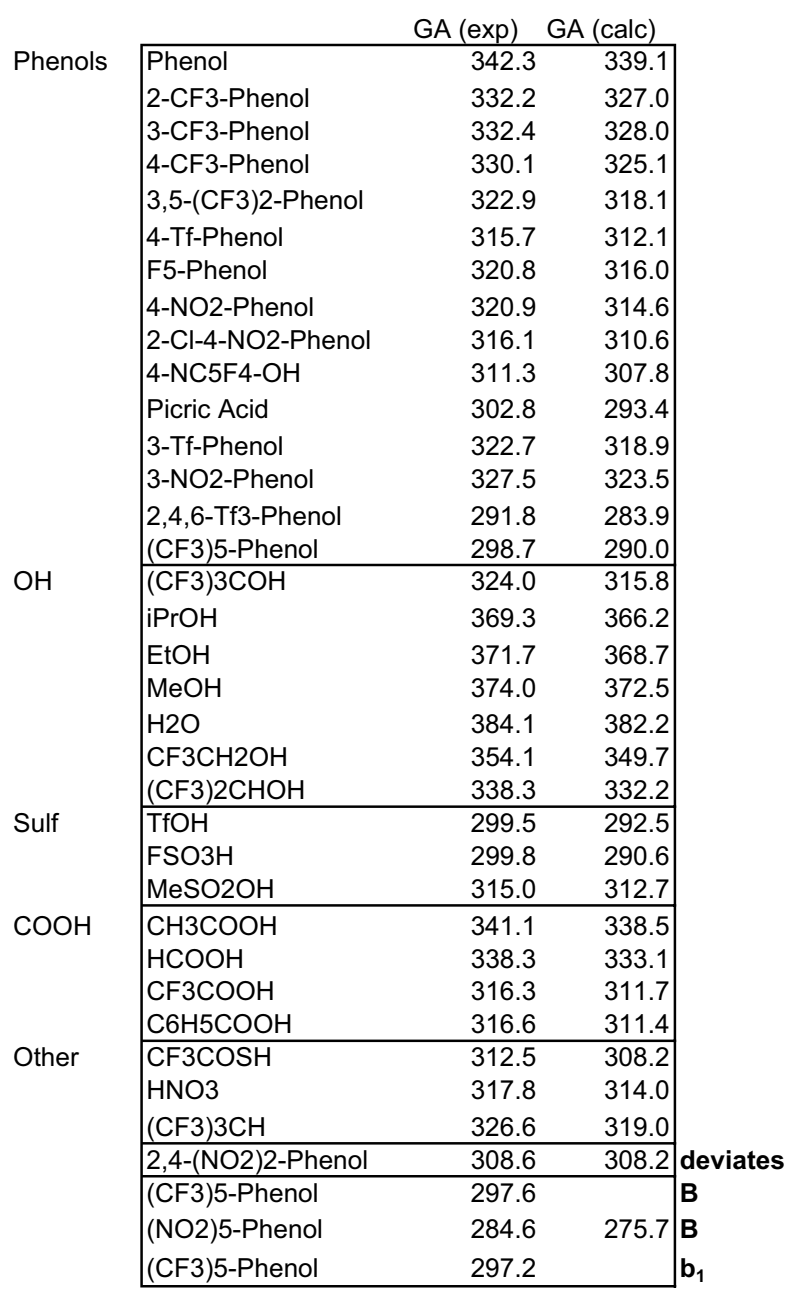

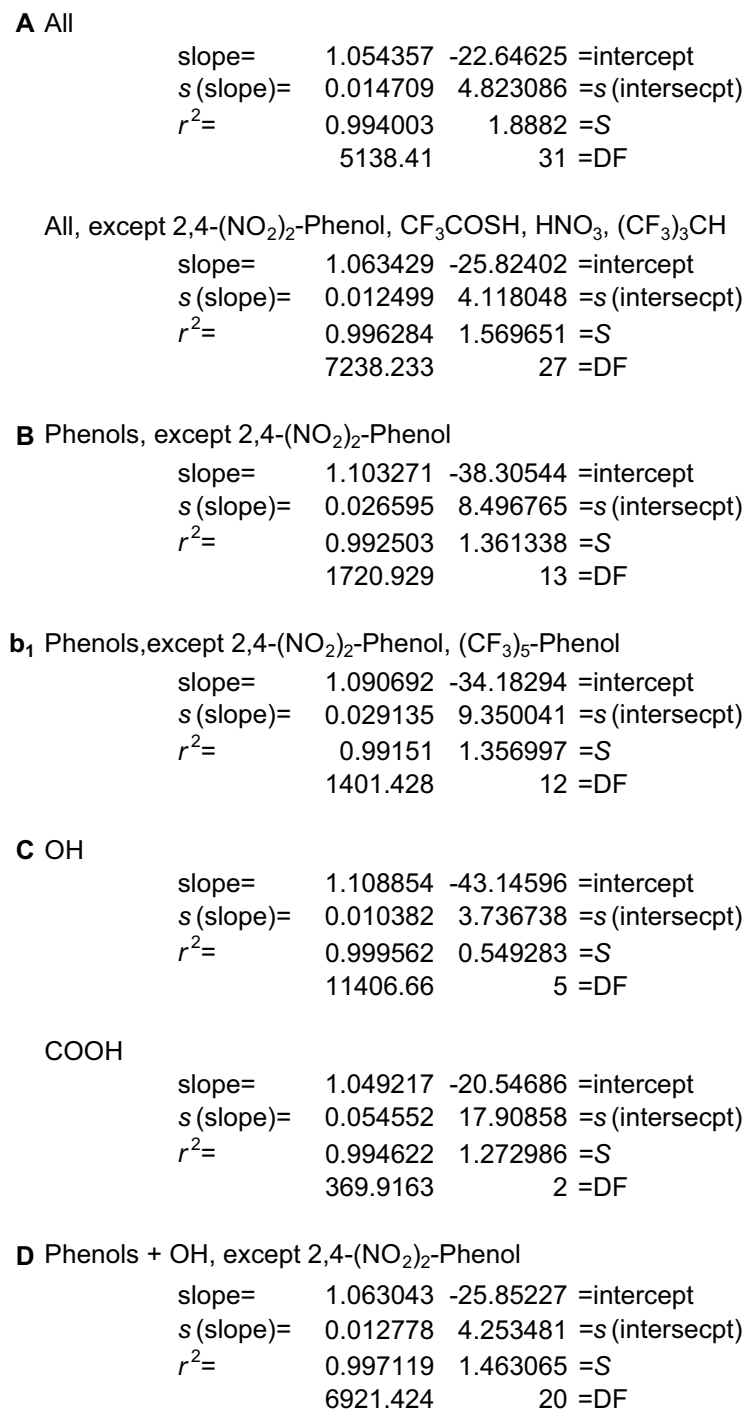

A All

b $_{1}$ Phenols, except 2,4-( $\left(\mathrm{NO}_{2}\right)_{2}$-Phenol, $\left(\mathrm{CF}_{3}\right)_{5}$-Phenol 


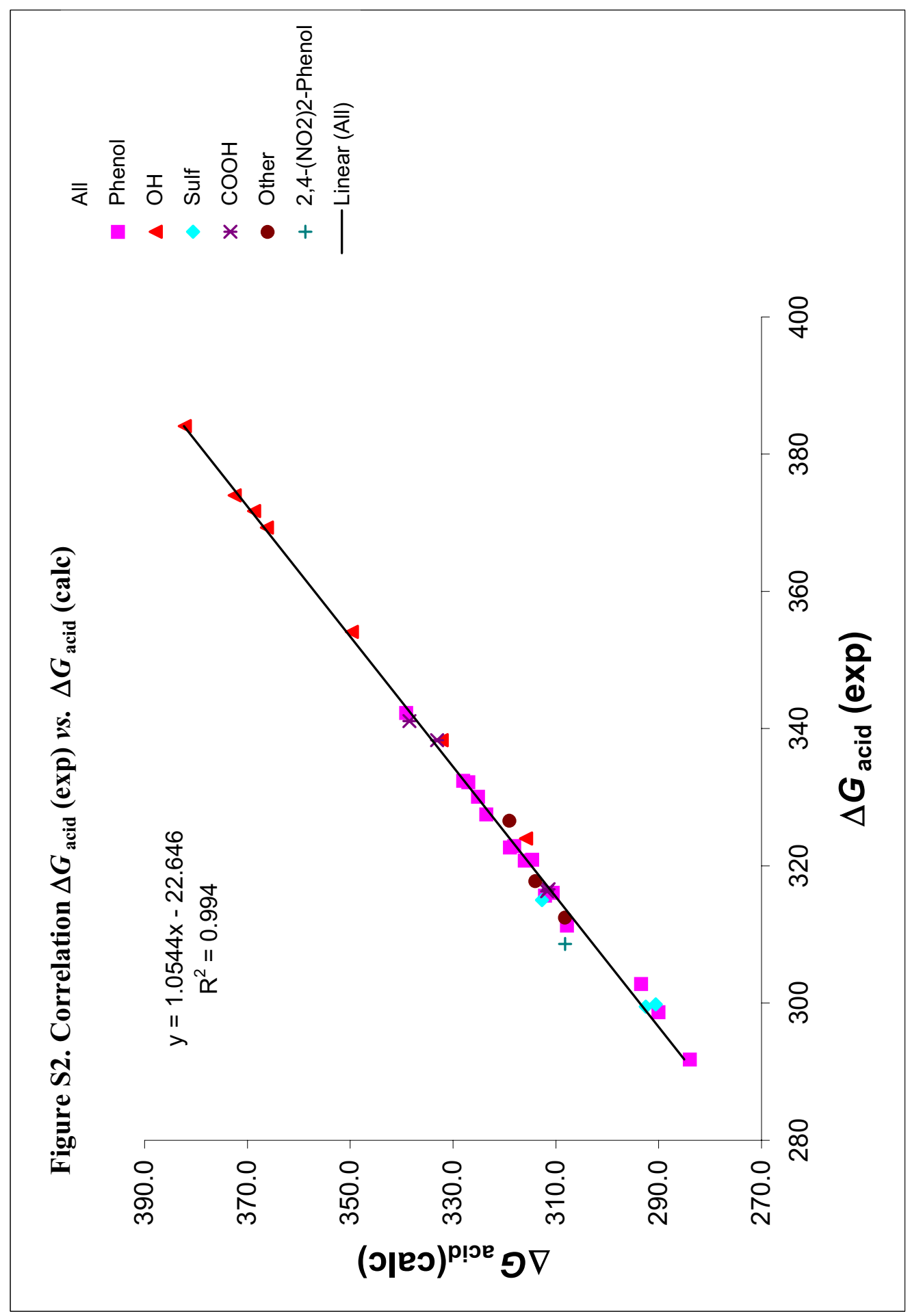


Table S4. Benzenes

\begin{tabular}{|c|c|c|c|c|c|c|c|}
\hline \multirow{13}{*}{$\begin{array}{l}\mathrm{CF}_{3} \text {-substituted } \\
\text { benzenes }\end{array}$} & & $\mathrm{GA}(\exp )$ & GA (calc) & & & & \\
\hline & Benzene & 390.9 & 393.2 & & $\mathbf{E}$ & All & \\
\hline & 4 & 379.0 & 378.1 & & slope $=$ & 0.954087 & $16.8862=$ intercept \\
\hline & 1,2 & 368.5 & 367.9 & & $s($ slope $)=$ & 0.039243 & $14.16667=s$ (intersecpt) \\
\hline & 1,3 & 365.0 & 363.4 & & $r^{2}=$ & 0.986646 & $2.100116=S$ \\
\hline & 1,4 & 366.6 & 366.5 & & & 591.0812 & $8=\mathrm{DF}$ \\
\hline & $1,2,3$ & 356.6 & 357.2 & & & & \\
\hline & $1,2,4$ & 355.4 & 354.3 & & & & \\
\hline & $1,3,5$ & 354.2 & 351.8 & & & Except & $1,2,3,4,5$ \\
\hline & $1,2,4,5$ & 341.4 & 344.3 & & slope $=$ & 1.004072 & $-1.58279=$ intercept \\
\hline & $1,2,3,4,5$ & 328.4 & 332.6 & & $s($ slope $)=$ & 0.045357 & $16.52969=s$ (intersecpt) \\
\hline & $1,2,3,4,5$ & 332.8 & & $e_{1}$ & $r^{2}=$ & 0.985917 & $1.876609=S$ \\
\hline & $1,2,3,4,5$ & 330.9 & & E & & 490.0598 & $7=\mathrm{DF}$ \\
\hline
\end{tabular}

Table S5. Toluenes

\begin{tabular}{|c|c|c|c|c|c|c|}
\hline & $\mathrm{GA}(\exp )$ & GA (calc) & & $\mathbf{F}$ & All & \\
\hline Toluene & 373.7 & 374.7 & & slope $=$ & 1.024747 & $7 \quad-9.49279=$ =intercept \\
\hline 3-CF3-Toluene & 362.0 & 362.9 & & $s($ slope $)=$ & 0.021915 & $57.379731=s$ (intersecpt) \\
\hline 4-CF3-Toluene & 359.8 & 357.6 & & $r^{2}=$ & 0.997263 & $1.71303=S$ \\
\hline 3,5-(CF3)2-Toluene & 354.3 & 351.9 & & & 2186.569 & $6=\mathrm{DF}$ \\
\hline TNT & 309.0 & 307.4 & & & & \\
\hline 2,3,4,5,6-(CN)5-Toluene & 302.0 & 301.8 & & & & \\
\hline 2,4,6-Tf3-Toluene & 305.7 & 301.6 & & $f_{1}$ & except & 2,3,4,5,6-(CF3)5-Toluene \\
\hline 2,3,4,5,6-(CF3)5-Toluene & 318.4 & 317.5 & & slope $=$ & 1.027178 & $3-10.4168=$ intercept \\
\hline 2-CF3-Toluene & 360.3 & 359.7 & $F$ & $s($ slope $)=$ & 0.024265 & $8.232708=s$ (intersecpt) \\
\hline 2,3,4,5,6-(CF3)5-Toluene & 319.1 & & $F$ & $r^{2}=$ & 0.997218 & $1.84342=S$ \\
\hline $2,3,4,5,6$-(CF3)5-Toluene & 319.2 & & $f_{1}$ & & 1792.029 & $5=\mathrm{DF}$ \\
\hline
\end{tabular}




\subsection{Calculation of $\Delta G$ and $\Delta H$ Values of Isodesmic Reactions}

Table S6. Calculation of $\Delta G$ Values of Isodesmic Reactions for Phenols.

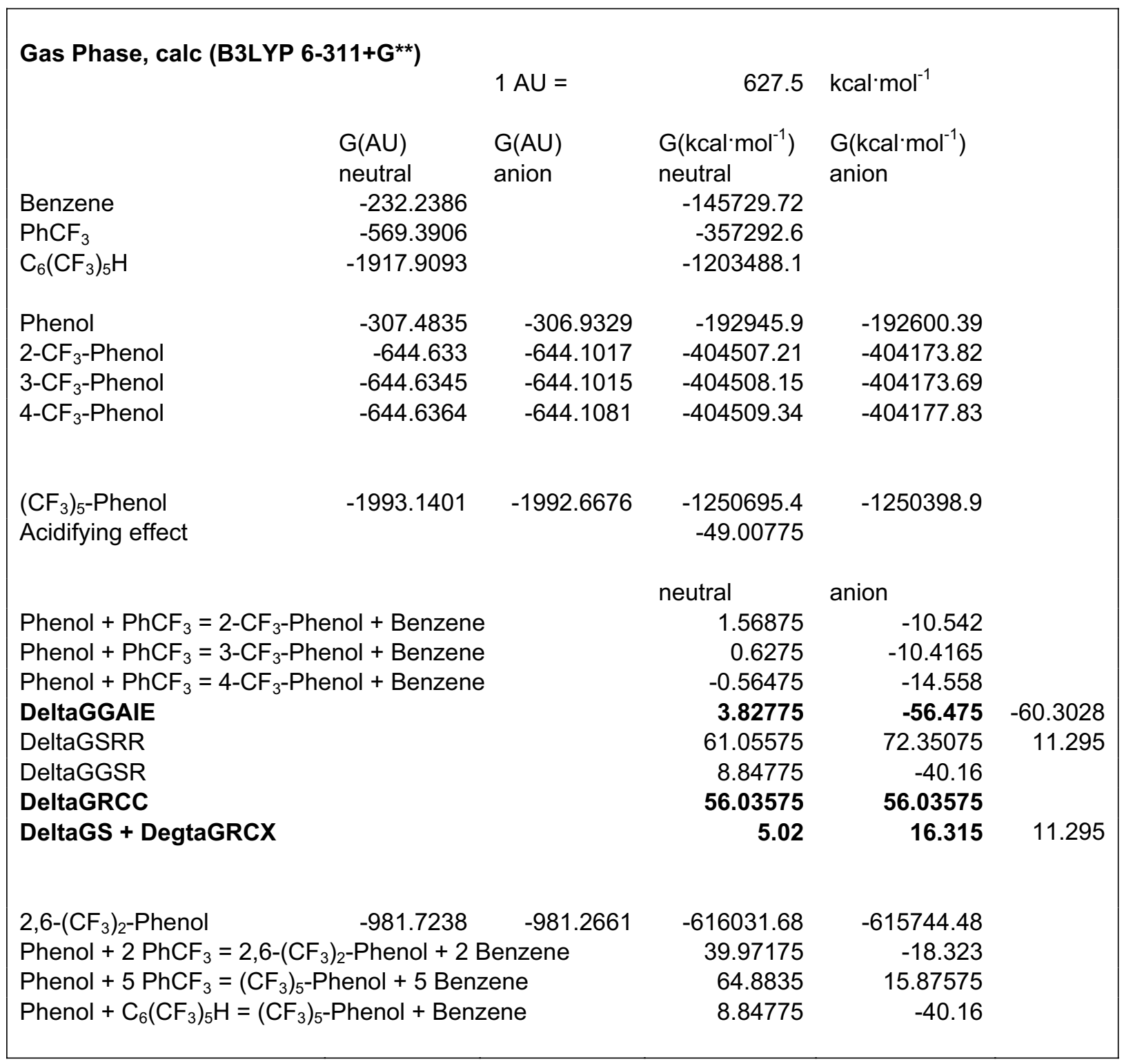


Table S7. Calculation of $\Delta H$ Values of Isodesmic Reactions for Phenols.

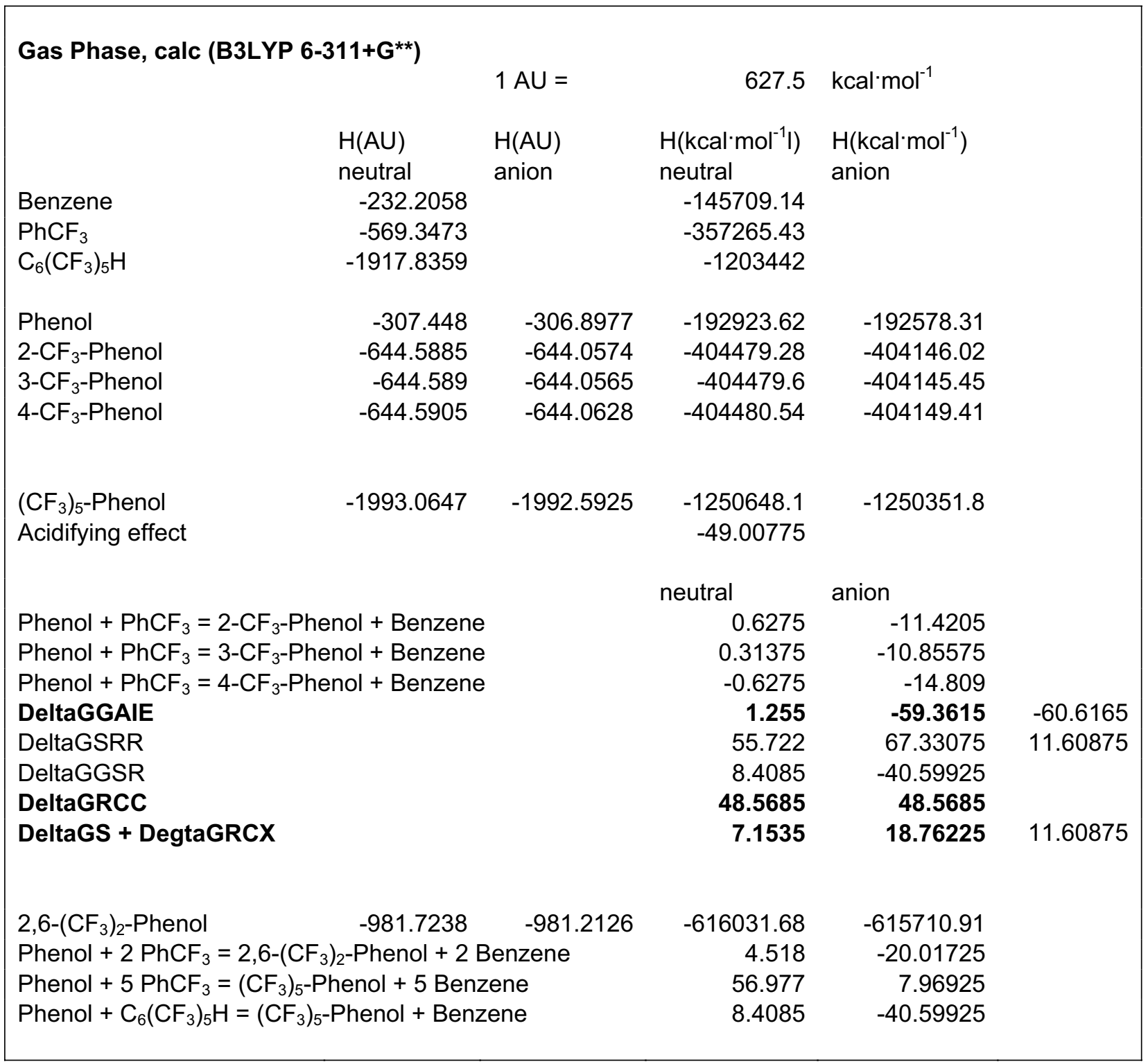


Table S8. Calculation of $\Delta G$ Values of Isodesmic Reactions for Anilines.

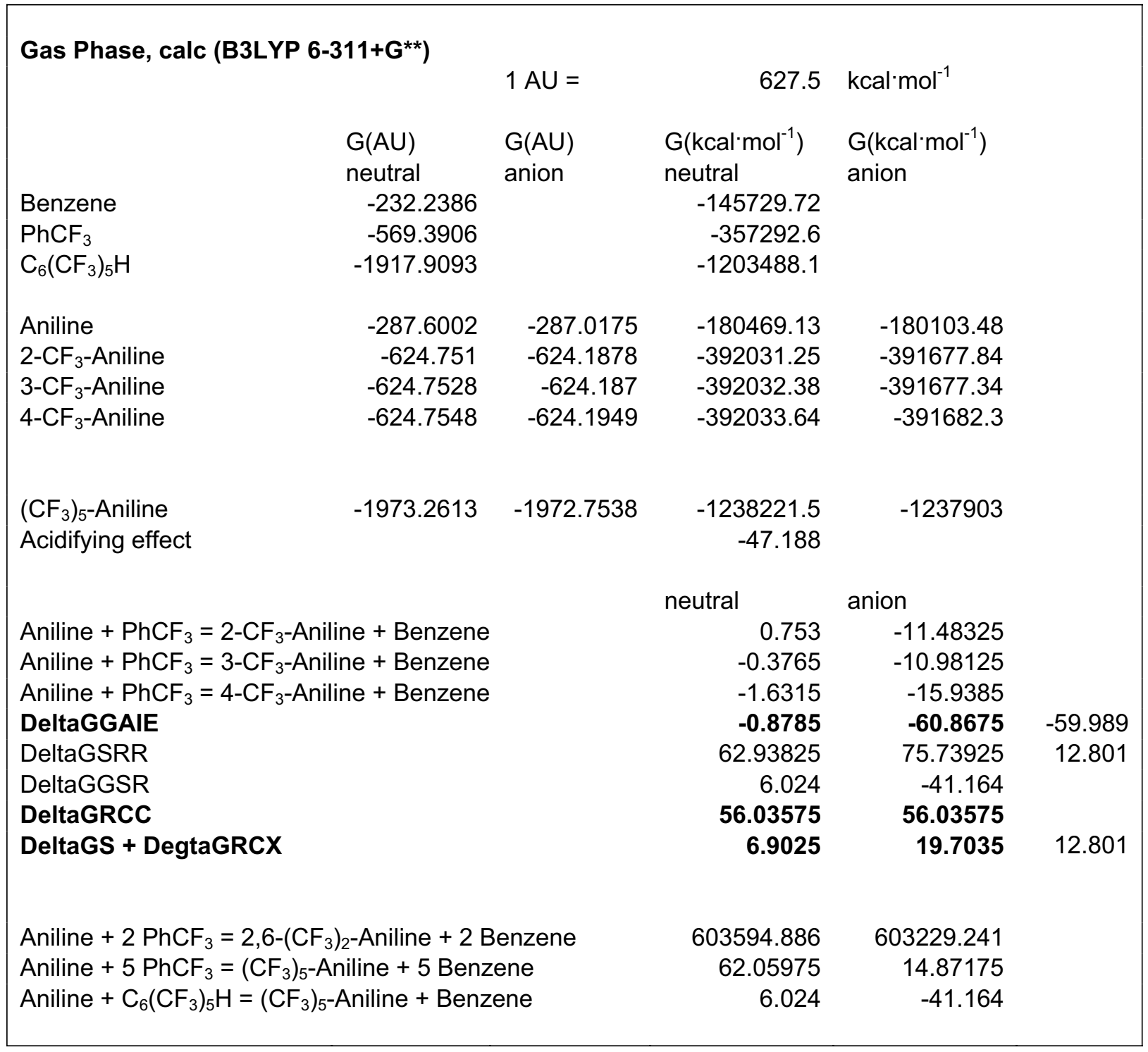


Table S9. Calculation of $\Delta H$ Values of Isodesmic Reactions for Anilines.

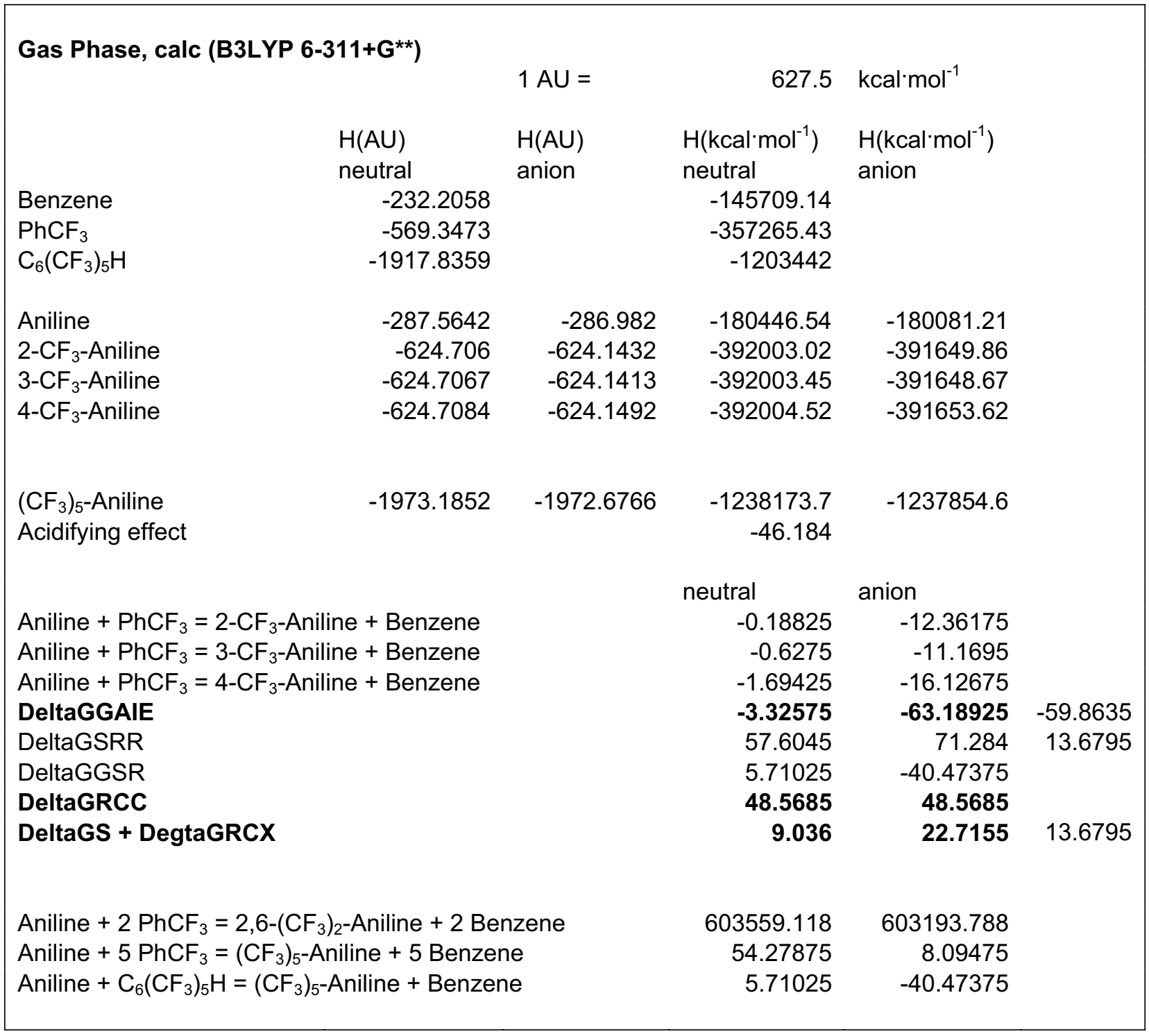


Table S10. Calculation of $\Delta G$ Values of Isodesmic Reactions for Toluenes.

\begin{tabular}{|c|c|c|c|c|c|}
\hline \multicolumn{6}{|c|}{ Gas Phase, calc (B3LYP 6-311+G**) } \\
\hline & $\begin{array}{l}\mathrm{G}(\mathrm{AU}) \\
\text { neutral }\end{array}$ & $\begin{array}{l}\mathrm{G}(\mathrm{AU}) \\
\text { anion }\end{array}$ & $\begin{array}{l}\mathrm{G}\left(\mathrm{kcal} \cdot \mathrm{mol}^{-1}\right) \\
\text { neutral }\end{array}$ & $\begin{array}{l}\mathrm{G}\left(\mathrm{kcal} \cdot \mathrm{mol}^{-1}\right) \\
\text { anion }\end{array}$ & \\
\hline Benzene & -232.2386 & & -145729.72 & & \\
\hline $\mathrm{PhCF}_{3}$ & -569.3906 & & -357292.6 & & \\
\hline $\mathrm{C}_{6}\left(\mathrm{CF}_{3}\right)_{5} \mathrm{H}$ & -1917.9093 & & -1203488.1 & & \\
\hline Toluene & -271.5431 & -270.9357 & -170393.3 & -170012.15 & \\
\hline 2-CF - -Toluene & -608.69 & -608.1065 & -381952.98 & -381586.83 & \\
\hline 3-CF - -Toluene & -608.6945 & -608.106 & -381955.8 & -381586.52 & \\
\hline 4- $\mathrm{CF}_{3}$-Toluene & -608.6954 & -608.1153 & -381956.36 & -381592.35 & \\
\hline \multirow[t]{2}{*}{$\begin{array}{l}\left(\mathrm{CF}_{3}\right)_{5} \text {-Toluene } \\
\text { Acidifying effect }\end{array}$} & -1957.1925 & -1956.6764 & $\begin{array}{r}-1228138.3 \\
-57.29075\end{array}$ & -1227814.4 & \\
\hline & & & neutral & anion & \\
\hline \multicolumn{3}{|c|}{ Toluene $+\mathrm{PhCF}_{3}=2-\mathrm{CF}_{3}$-Toluene + Benzene } & 3.20025 & -11.797 & \\
\hline \multicolumn{3}{|c|}{ Toluene $+\mathrm{PhCF}_{3}=3-\mathrm{CF}_{3}$-Toluene + Benzene } & 0.3765 & -11.48325 & \\
\hline \multicolumn{3}{|c|}{ Toluene $+\mathrm{PhCF}_{3}=4-\mathrm{CF}_{3}$-Toluene + Benzene } & -0.18825 & -17.319 & \\
\hline \multicolumn{3}{|c|}{ DeltaGGAIE } & 6.96525 & -63.8795 & -70.8447 \\
\hline \multicolumn{3}{|l|}{ DeltaGSRR } & 62.43625 & 75.99025 & 13.554 \\
\hline \multicolumn{3}{|l|}{ DeltaGGSR } & 13.36575 & -43.925 & \\
\hline \multicolumn{3}{|l|}{ DeltaGRCC } & 56.03575 & 56.03575 & \\
\hline \multicolumn{3}{|c|}{ DeltaGS + DegtaGRCX } & 6.4005 & 19.9545 & 13.554 \\
\hline \multicolumn{3}{|c|}{ Acidifying effect (check) } & -57.29075 & & \\
\hline \multirow{3}{*}{\multicolumn{3}{|c|}{$\begin{array}{l}\text { Toluene + } 2 \mathrm{PhCF}_{3}=2,6-\left(\mathrm{CF}_{3}\right)_{2} \text {-Toluene }+2 \text { Benzene } \\
\text { Toluene }+5 \mathrm{PhCF}_{3}=\left(\mathrm{CF}_{3}\right)_{5} \text {-Toluene }+5 \text { Benzene } \\
\text { Toluene }+\mathrm{C}_{6}\left(\mathrm{CF}_{3}\right)_{5} \mathrm{H}=\left(\mathrm{CF}_{3}\right)_{5} \text {-Toluene }+ \text { Benzene }\end{array}$}} & 593519.055 & 593137.912 & \\
\hline & & & 69.4015 & 12.11075 & \\
\hline & & & 13.36575 & -43.925 & \\
\hline
\end{tabular}


Table S11. Calculation of $\Delta H$ Values of Isodesmic Reactions for Toluenes.

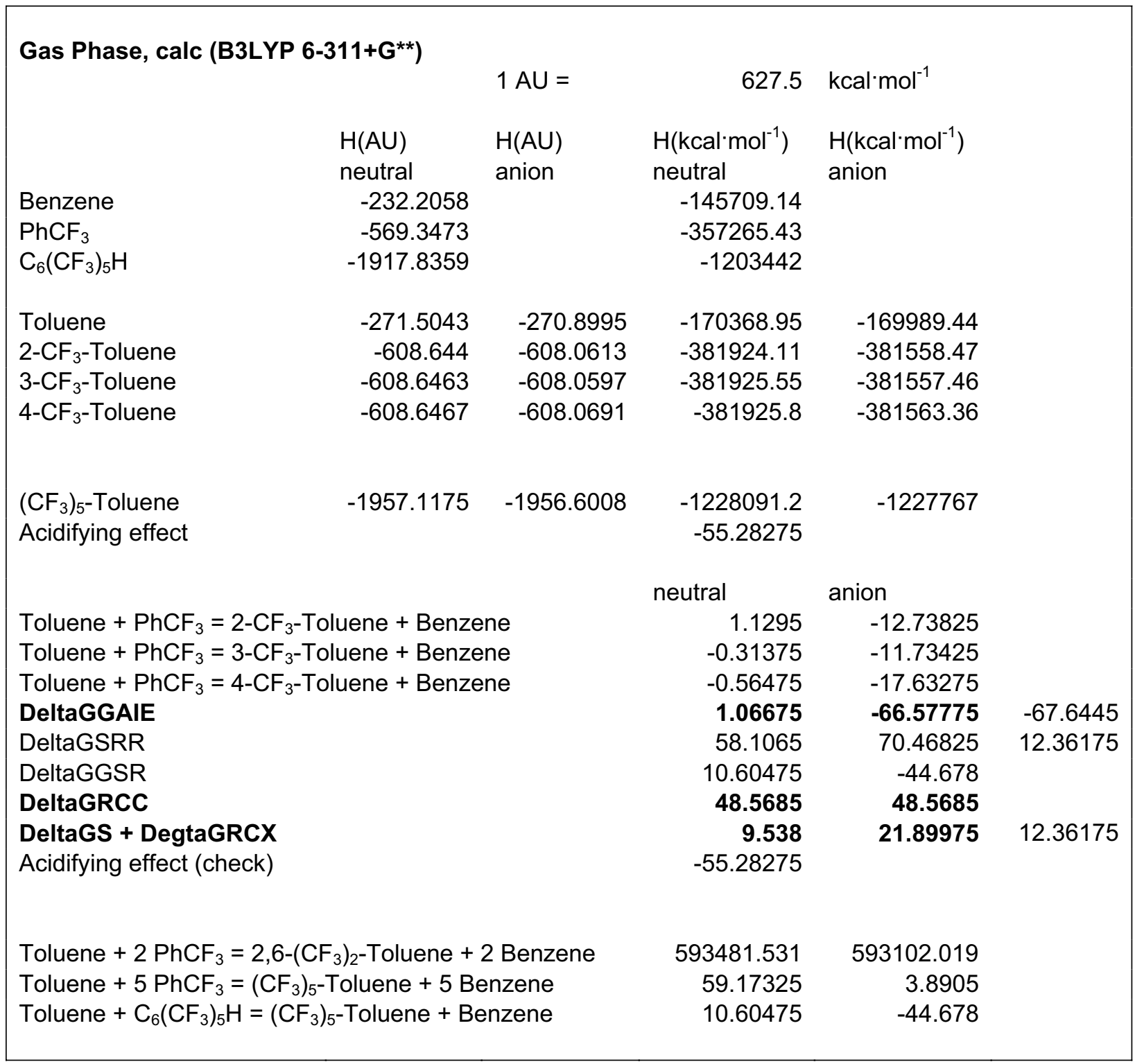


Table S12. Calculation of $\Delta G$ Values of Isodesmic Reactions for Benzenes.

\begin{tabular}{|c|c|c|c|c|c|}
\hline \multicolumn{6}{|c|}{ Gas Phase, calc (B3LYP 6-311+G**) } \\
\hline & & $1 \mathrm{AU}=$ & 627.5 & \multicolumn{2}{|l|}{$\mathrm{kcal} \cdot \mathrm{mol}^{-1}$} \\
\hline & $\begin{array}{l}\mathrm{G}(\mathrm{AU}) \\
\text { neutral }\end{array}$ & $\begin{array}{l}\mathrm{G}(\mathrm{AU}) \\
\text { anion }\end{array}$ & $\begin{array}{l}\mathrm{G}\left(\mathrm{kcal}^{\prime} \cdot \mathrm{mol}^{-1}\right) \\
\text { neutral }\end{array}$ & \multicolumn{2}{|l|}{$\begin{array}{l}\mathrm{G}\left(\mathrm{kcal} \cdot \mathrm{mol}^{-1}\right) \\
\text { anion }\end{array}$} \\
\hline Benzene & -232.2386 & & -145729.72 & & \\
\hline $\mathrm{PhCF}_{3}$ & -569.3906 & & -357292.6 & & \\
\hline $\mathrm{C}_{6}\left(\mathrm{CF}_{3}\right)_{5} \mathrm{H}$ & -1917.9093 & & -1203488.1 & & \\
\hline Benzene & -232.2386 & -231.6018 & -145729.72 & \multicolumn{2}{|l|}{-145330.13} \\
\hline 2-CF -Benzene & -569.3906 & -568.7779 & -357292.6 & \multicolumn{2}{|l|}{-356908.13} \\
\hline 3-CF - -Benzene & -569.3906 & -568.7738 & -357292.6 & \multicolumn{2}{|l|}{-356905.56} \\
\hline 4-CF - -Benzene & -569.3906 & -568.7748 & -357292.6 & \multicolumn{2}{|l|}{-356906.19} \\
\hline \multirow[t]{2}{*}{$\begin{array}{l}\left(\mathrm{CF}_{3}\right)_{5} \text {-Benzene } \\
\text { Acidifying effect }\end{array}$} & -1917.9093 & -1917.3696 & $\begin{array}{r}-1203488.1 \\
-60.93025\end{array}$ & \multicolumn{2}{|l|}{-1203149.4} \\
\hline & & & neutral & \multicolumn{2}{|l|}{ anion } \\
\hline \multicolumn{3}{|c|}{ Benzene $+\mathrm{PhCF}_{3}=2-\mathrm{CF}_{3}$-Benzene + Benzene } & 0 & \multicolumn{2}{|l|}{-15.12275} \\
\hline \multicolumn{3}{|c|}{ Benzene $+\mathrm{PhCF}_{3}=3-\mathrm{CF}_{3}$-Benzene + Benzene } & 0 & \multicolumn{2}{|l|}{-12.55} \\
\hline \multicolumn{3}{|c|}{ Benzene $+\mathrm{PhCF}_{3}=4-\mathrm{CF}_{3}$-Benzene + Benzene } & 0 & \multicolumn{2}{|l|}{-13.1775} \\
\hline \multicolumn{3}{|l|}{ DeltaGGAIE } & 0 & -68.523 & -68.523 \\
\hline \multicolumn{3}{|l|}{ DeltaGSRR } & 56.03575 & \multirow{2}{*}{\multicolumn{2}{|c|}{$\begin{array}{r}63.6285 \\
-60.93025\end{array}$}} \\
\hline \multicolumn{3}{|l|}{ DeltaGGSR } & 0 & & \\
\hline \multicolumn{3}{|l|}{ DeltaGRCC } & 56.03575 & \multicolumn{2}{|l|}{56.03575} \\
\hline \multicolumn{3}{|c|}{ DeltaGS + DegtaGRCX } & 0 & \multirow[t]{2}{*}{7.59275} & 7.59275 \\
\hline Acidifying effect & & & -60.93025 & & \\
\hline
\end{tabular}


Table S13. Calculation of $\Delta H$ Values of Isodesmic Reactions for Benzenes.

\begin{tabular}{|c|c|c|c|c|c|}
\hline \multicolumn{6}{|c|}{ Gas Phase, calc (B3LYP 6-311+G**) } \\
\hline & $\begin{array}{l}\mathrm{H}(\mathrm{AU}) \\
\text { neutral }\end{array}$ & $\begin{array}{l}\mathrm{H}(\mathrm{AU}) \\
\text { anion }\end{array}$ & $\begin{array}{l}\mathrm{H}\left(\mathrm{kcal} \cdot \mathrm{mol}^{-1}\right) \\
\text { neutral }\end{array}$ & $\begin{array}{l}\mathrm{H}\left(\mathrm{kcal} \cdot \mathrm{mol}^{-1}\right) \\
\text { anion }\end{array}$ & \\
\hline Benzene & -232.2058 & & -145709.14 & & \\
\hline $\mathrm{PhCF}_{3}$ & -569.3473 & & -357265.43 & & \\
\hline $\mathrm{C}_{6}\left(\mathrm{CF}_{3}\right)_{5} \mathrm{H}$ & -1917.8359 & & -1203442 & & \\
\hline Benzene & -232.2058 & -231.5696 & -145709.14 & -145309.92 & \\
\hline 2-CF - -Benzene & -569.3473 & -568.7352 & -357265.43 & -356881.34 & \\
\hline 3-CF - -Benzene & -569.3473 & -568.7305 & -357265.43 & -356878.39 & \\
\hline $4-\mathrm{CF}_{3}$-Benzene & -569.3473 & -568.7313 & -357265.43 & -356878.89 & \\
\hline \multirow[t]{2}{*}{$\begin{array}{l}\left(\mathrm{CF}_{3}\right)_{5} \text {-Benzene } \\
\text { Acidifying effect }\end{array}$} & -1917.8359 & -1917.2934 & $\begin{array}{l}-1203442 \\
-58.79675\end{array}$ & -1203101.6 & \\
\hline & & & neutral & anion & \\
\hline \multicolumn{3}{|c|}{ Benzene $+\mathrm{PhCF}_{3}=2-\mathrm{CF}_{3}$-Benzene + Benzene } & 0 & -15.12275 & \\
\hline \multicolumn{3}{|c|}{ Benzene $+\mathrm{PhCF}_{3}=3-\mathrm{CF}_{3}$-Benzene + Benzene } & 0 & -12.1735 & \\
\hline \multicolumn{3}{|c|}{ Benzene $+\mathrm{PhCF}_{3}=4-\mathrm{CF}_{3}$-Benzene + Benzene } & 0 & -12.6755 & \\
\hline \multicolumn{3}{|c|}{ DeltaGGAIE } & 0 & -67.268 & -67.268 \\
\hline \multicolumn{3}{|l|}{ DeltaGSRR } & 48.5685 & 57.03975 & 8.47125 \\
\hline \multicolumn{3}{|l|}{ DeltaGGSR } & 0 & -58.79675 & \\
\hline \multicolumn{3}{|l|}{ DeltaGRCC } & 48.5685 & 48.5685 & \\
\hline \multicolumn{3}{|c|}{ DeltaGS + DegtaGRCX } & 0 & 8.47125 & 8.47125 \\
\hline \multicolumn{3}{|c|}{ Acidifying effect (check) } & -58.79675 & & \\
\hline
\end{tabular}




\subsection{Calculation of Additivities of $\mathrm{pK}_{a}$ Values and $\Delta G_{\text {acid }}$ Values}

Table S14. Phenols

Gas Phase, calc (B3LYP 6-311+G**):

\begin{tabular}{|lrrrr|}
\multicolumn{1}{c}{ GA(Calc) } & Acidifying eff & GA(Predicted) & \% Additivity \\
Phenol & 339.2 & & & \\
2-CF3 & 327.1 & 12.10 & & \\
4-CF3 & 328.2 & 11.00 & & \\
& 325.2 & 14.00 & & \\
2,6-(CF3)2 & & & 317.2 & $95.00 \%$ \\
(CF3)5C6OH & 318.3 & & $\mathbf{2 7 9}$ & $\mathbf{8 1 . 4 0 \%}$ \\
\hline
\end{tabular}

Gas Phase, exp:

\begin{tabular}{|lrrrr|}
\multicolumn{1}{c}{ GA(Exp) Acidifying eff } & GA(Predicted) & \% Additivity \\
\hline Phenol & 342.3 & & & \\
3-CF3 & 332.2 & 10.10 & & \\
4-CF3 & 332.4 & 9.90 & & \\
& 330.1 & 12.20 & & \\
3,5-(CF3)2 & & & & \\
(CF3)5C6OH & 322.9 & & 322.5 & $\mathbf{8 3 . 9 8 \%}$ \\
\hline
\end{tabular}

MeCN:

\begin{tabular}{|lrrrr|}
\multicolumn{1}{c}{} & pKa(exp, used) & Acidifying eff & pKa(predicted) & \% Additivity \\
\hline Phenol & 29.14 & & & \\
2-CF3 & 24.88 & 4.26 & & \\
3-CF3 & 26.50 & 2.64 & & \\
4-CF3 & 25.54 & 3.60 & & \\
& & & & \\
3,5-(CF3)2 & 23.78 & & 23.86 & $101.52 \%$ \\
(CF3)5C6OH & $\mathbf{1 0 . 4 6}$ & & $\mathbf{1 1 . 7 4}$ & $\mathbf{1 0 7 . 3 6 \%}$ \\
\hline
\end{tabular}

DMSO:

\begin{tabular}{|lrrrr|}
\multicolumn{1}{c}{} & $\mathrm{pKa}(\mathrm{exp}$, used) & Acidifying eff & $\mathrm{pKa}$ (predicted) & \% Additivity \\
\hline Phenol & 18.0 & & & \\
2-CF3 & 14.4 & 3.60 & & \\
3-CF3 & 15.1 & 2.90 & & \\
4-CF3 & 14.6 & 3.40 & & \\
& & & & \\
3,5-(CF3)2 & 13.2 & & 12.2 & $\mathbf{9 2 . 7 6 \%}$ \\
(CF3)5C6OH & $\mathbf{3 . 1}$ & & $\mathbf{1 . 6}$ & $\mathbf{9 0 . 8 5 \%}$ \\
\hline
\end{tabular}

H2O:

\begin{tabular}{|lrrrr|}
\multicolumn{1}{c}{} & $\mathrm{pKa}(\mathrm{exp}, \mathrm{used})$ Acidifying eff & $\mathrm{pKa}$ (predicted) & \% Additivity \\
\hline Phenol & 9.95 & & & \\
2-CF3 & 8.4 & 1.55 & & \\
3-CF3 & 8.95 & 1.00 & & \\
4-CF3 & 8.675 & 1.28 & & \\
& & & & \\
3,5-(CF3)2 & 8.26 & & 8.0 & $\mathbf{1 3 5 . 5 0 \%}$ \\
(CF3)5C6OH & 1.32 & & 3.575 & $137 \%$ \\
\hline
\end{tabular}


Table S15. Anilines

Gas Phase, calc (B3LYP 6-311+G**):

\begin{tabular}{|lrrrr|}
\multicolumn{1}{c}{ GA(Calc) Acidifying eff } & GA(Predicted) & \% Additivity \\
\hline Aniline & 359.4 & & & \\
3-CF3 & 347.1 & 12.3 & & \\
4-CF3 & 348.8 & 10.6 & & \\
& 345.1 & 14.3 & & \\
(CF3)5 & 312.2 & & 299.3 & $78.54 \%$ \\
\hline
\end{tabular}

Gas Phase, exp:

\begin{tabular}{|lrrrr|}
\multicolumn{1}{c}{ GA(Exp) Acidifying eff } & GA(Predicted) & \% Additivity \\
\hline Aniline & 359.1 & & & \\
2-CF3 & 347.1 & 12 & & \\
4-CF3 & 349.6 & 9.5 & & \\
& 346.0 & 13.1 & & \\
(CF3)5 & & & 303 & $86.19 \%$ \\
\hline
\end{tabular}

Table S16. Toluenes

Gas Phase, calc (B3LYP 6-311+G**):

\begin{tabular}{|lrrrr|} 
& GA(Calc) Acidifying eff & GA(Predicted) & \% Additivity \\
\hline Toluene & 374.9 & & & \\
2-CF3 & 359.9 & 15 & & \\
4-CF3 & 363.0 & 11.9 & & \\
& 357.7 & 17.2 & & \\
(CF3)5 & 317.6 & & 303.9 & $80.70 \%$ \\
\hline
\end{tabular}

Gas Phase, exp:

\begin{tabular}{|lrrrr|}
\multicolumn{1}{c}{ GA(Exp) Acidifying eff } & GA(Predicted) & \% Additivity \\
\hline Toluene & 373.7 & & & \\
3-CF3 & 359.9 & 13.8 & & \\
4-CF3 & 362.0 & 11.7 & & \\
& 359.8 & 13.9 & & \\
(CF3)5 & 318.9 & & 308.8 & $84.44 \%$ \\
\hline
\end{tabular}

\section{Table S17. Benzenes}

Gas Phase, calc (B3LYP 6-311+G**):

\begin{tabular}{|lcccc|}
\multicolumn{1}{c}{$\mathrm{GA}($ Calc) } & Acidifying eff & GA(Predicted) & \% Additivity \\
\hline Benzene & 393.3 & & & \\
2-CF3 & 378.2 & 15.1 & & \\
4-CF3 & 380.8 & 12.5 & & \\
& 380.1 & 13.2 & & \\
$(\mathrm{CF} 3) 5$ & 332.4 & & 324.9 & $89.04 \%$ \\
\hline
\end{tabular}


Table S18. Fluorine Substituted Phenols

H2O:

\begin{tabular}{|lrrrr|}
\multicolumn{1}{c}{} & pKa(exp, used) Acidifying eff & pKa(predicted) & \% Additivity \\
\hline Phenol & 9.95 & & & \\
$2-\mathrm{F}$ & 8.7 & 1.25 & & \\
$3-\mathrm{F}$ & 9.3 & 0.65 & & \\
$4-\mathrm{F}$ & 9.9 & 0.05 & & $115.58 \%$ \\
& & & 6.1 & \\
C6F5OH & 5.5 & & & \\
\hline
\end{tabular}

Gas Phase:

\begin{tabular}{|lcccc|}
\multicolumn{1}{c}{ GA(Exp) Acidifying eff } & GA(Predicted) & \% Additivity \\
\hline Phenol & 342.3 & & & \\
3-F & 339.0 & 3.3 & & \\
4-F & 336.8 & 5.5 & & \\
& 339.3 & 3.0 & & \\
C6F5OH & 320.0 & & 321.7 & $108.25 \%$ \\
\hline
\end{tabular}

DMSO:

\begin{tabular}{|lrrrr|}
\multicolumn{1}{c}{} & pKa(exp, used) Acidifying eff & pKa(predicted) & \% Additivity \\
\hline Phenol & 18.0 & & & \\
$2-\mathrm{F}$ & 15.6 & 2.4 & & \\
$3-\mathrm{F}$ & 15.9 & 2.1 & & \\
$4-\mathrm{F}$ & 18.1 & -0.1 & & $102.25 \%$ \\
& & & 9.1 & \\
$\mathrm{C} 6 \mathrm{~F} 5 \mathrm{OH}$ & 8.9 & & & \\
\hline
\end{tabular}




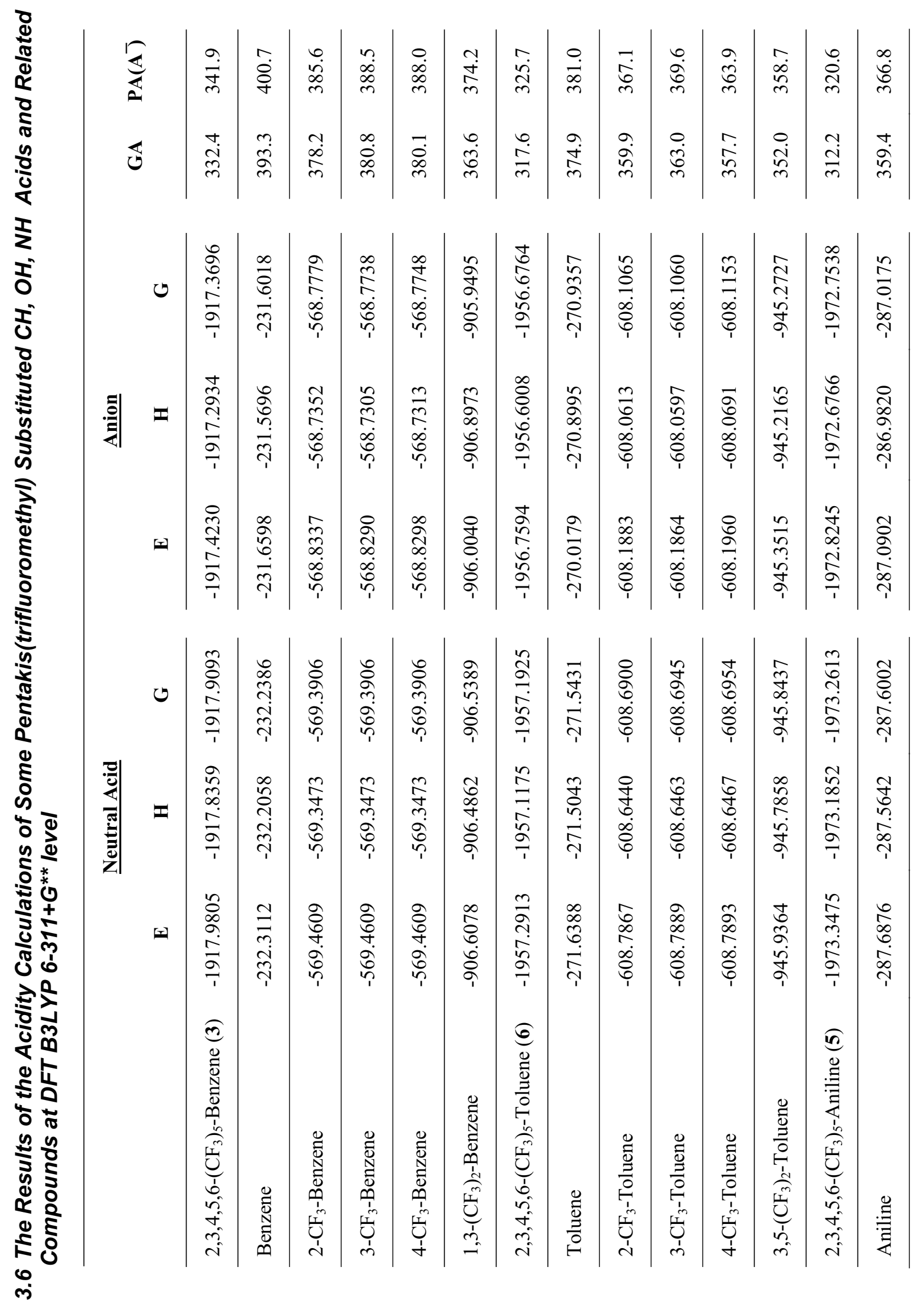




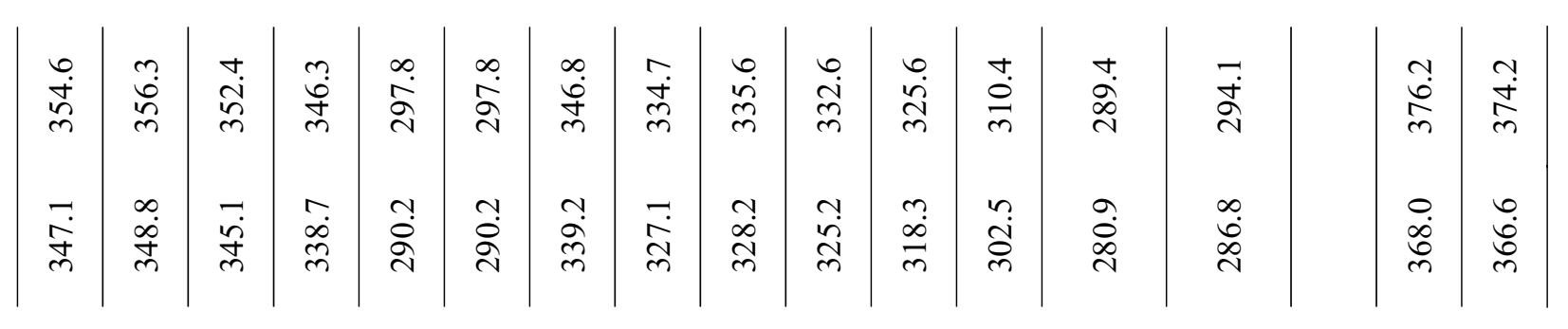

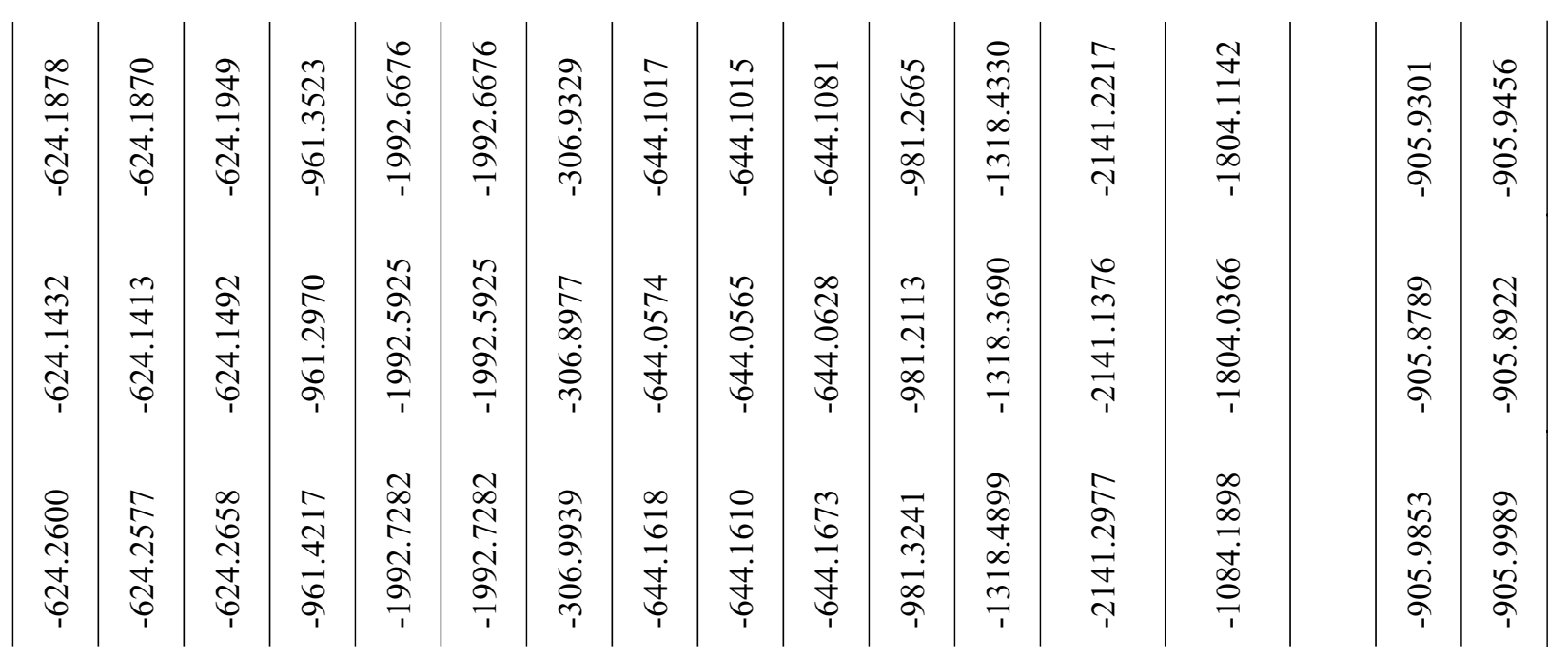

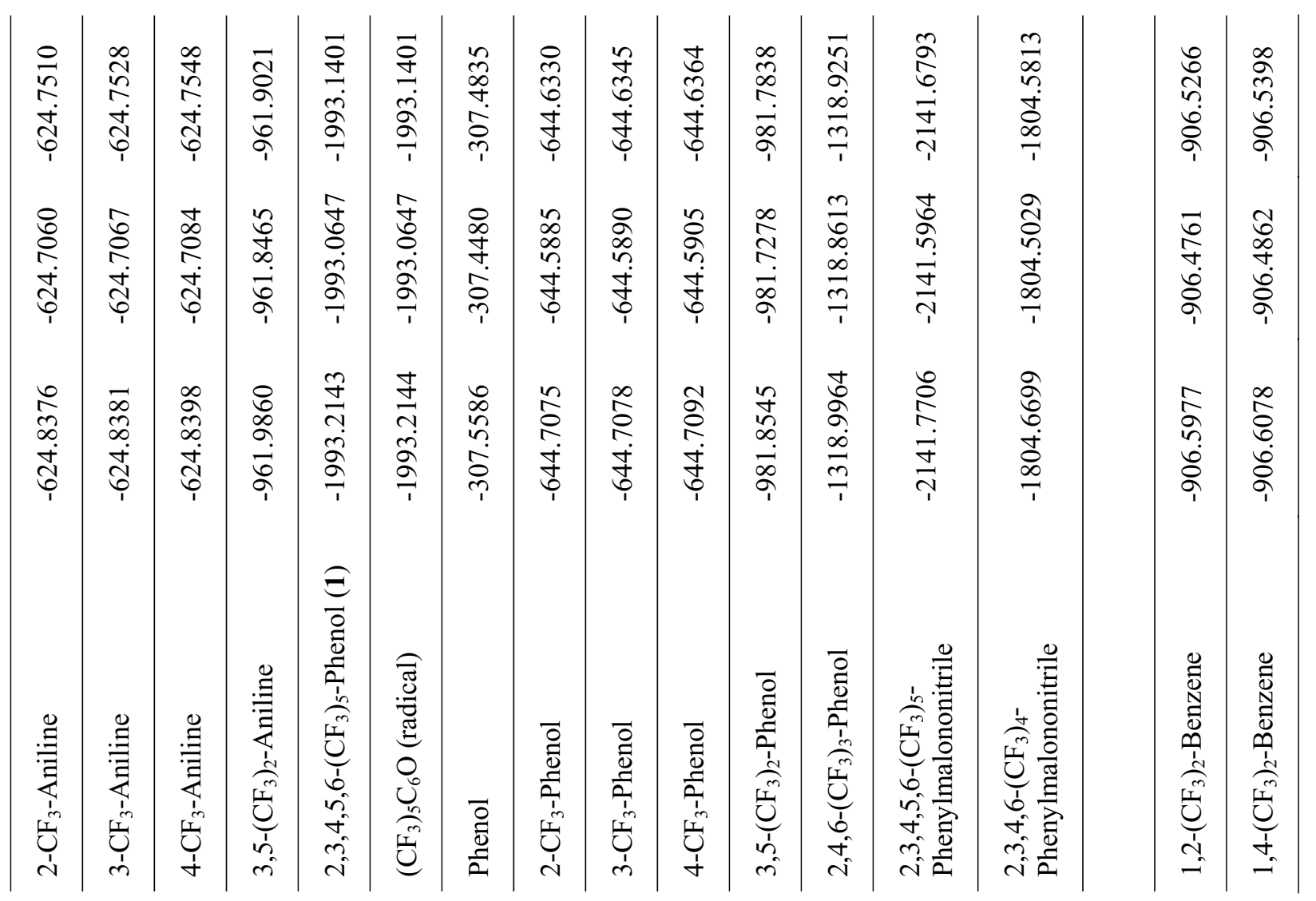




\begin{tabular}{|c|c|}
\hline $\begin{array}{l}0 \\
0\end{array}$ & 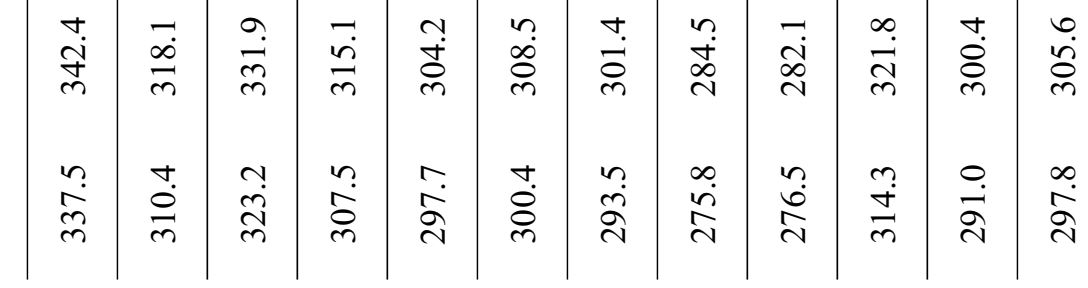 \\
\hline 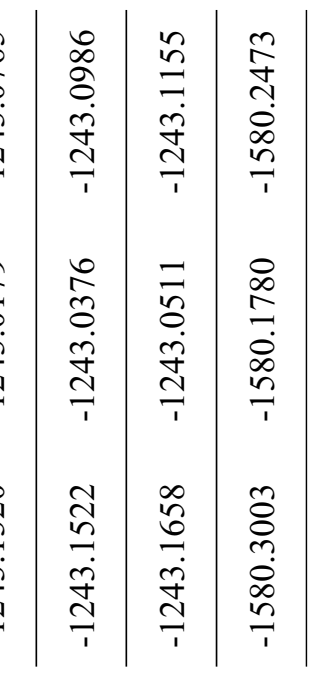 & 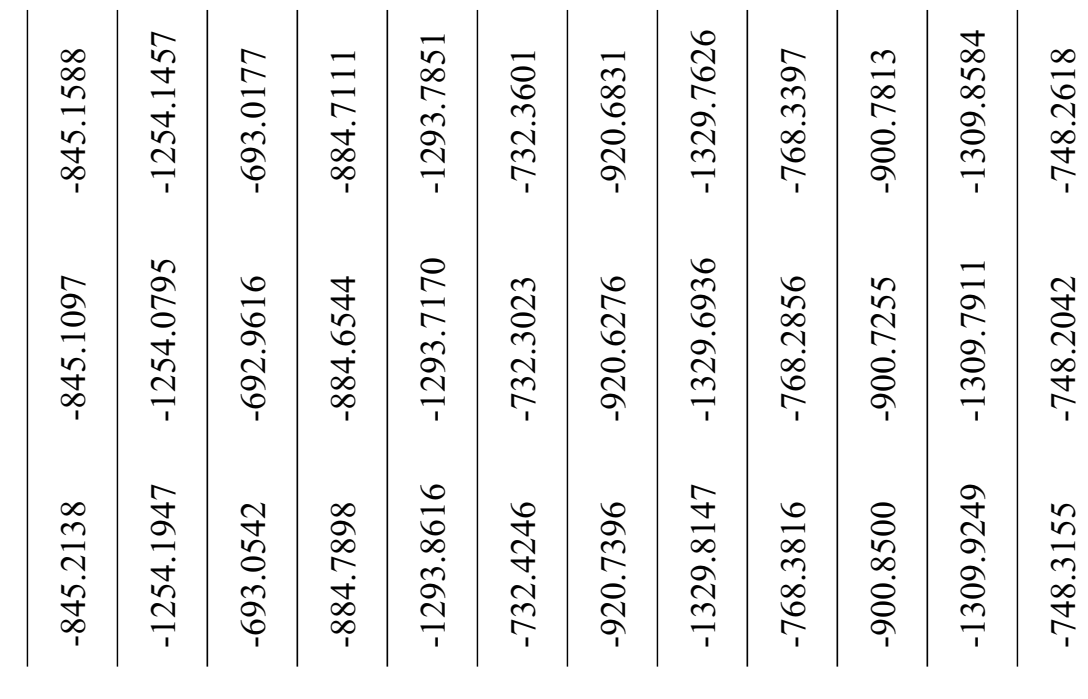 \\
\hline 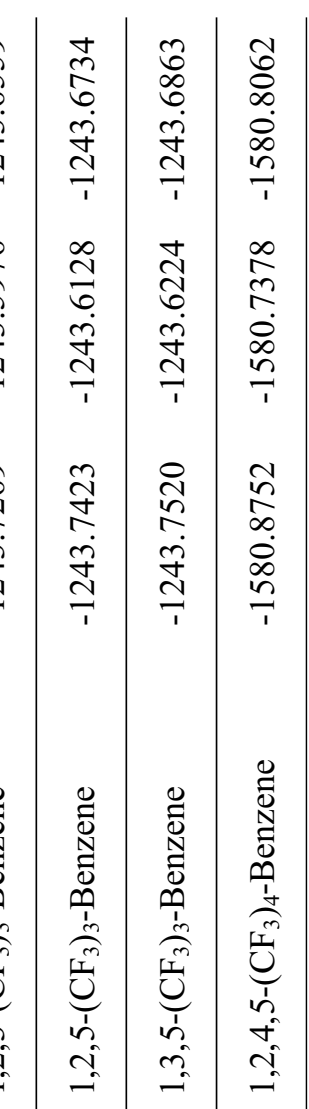 & 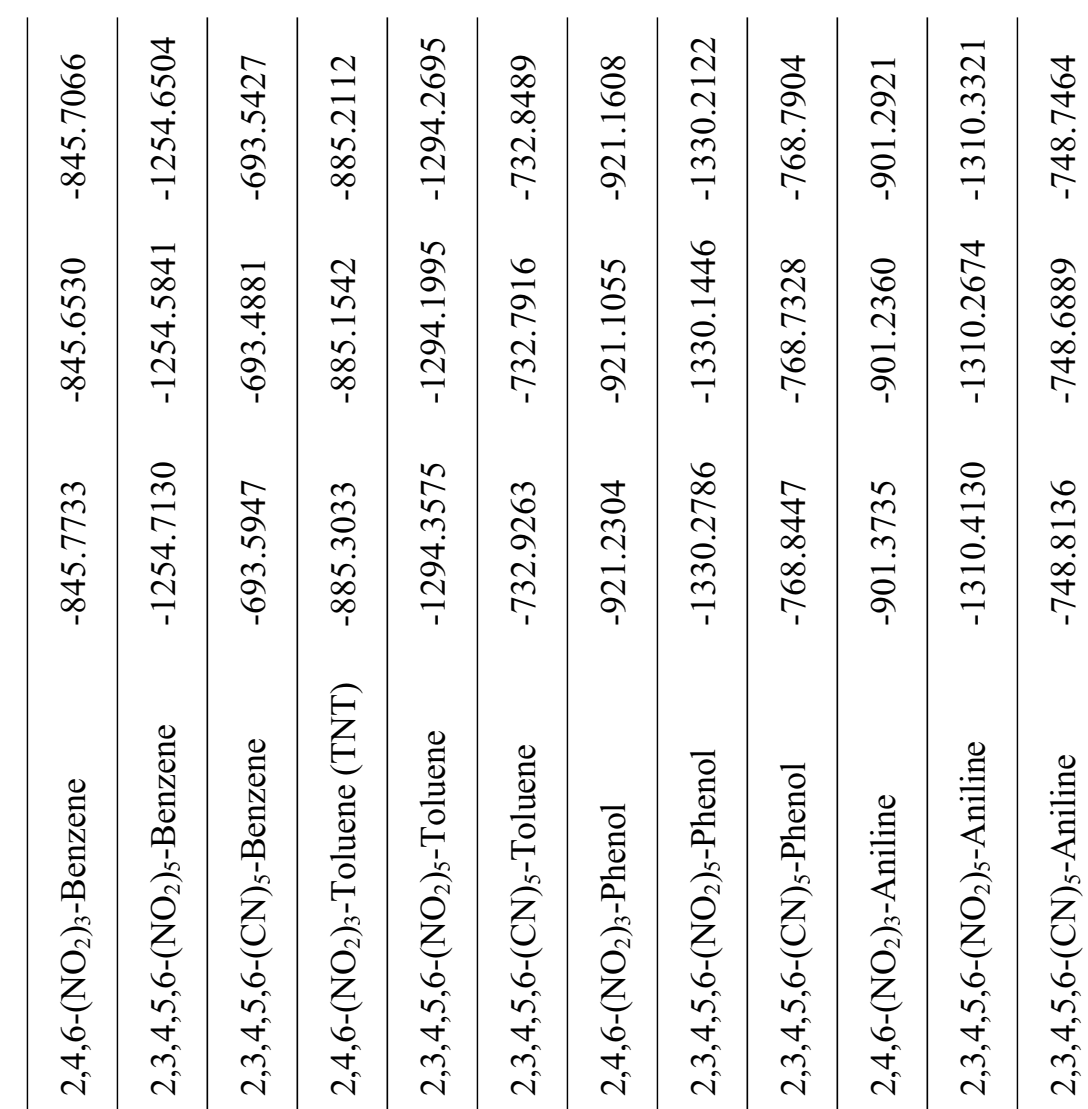 \\
\hline
\end{tabular}




\section{X-Ray Diffraction Crystal Data}

Table S19. Crystallographic data and structure refinement for compounds $1 \mathrm{a} 1 \cdot \mathrm{Me}_{4} \mathbf{N}^{+}, 1 \mathrm{a} \cdot \mathrm{Me}_{4} \mathbf{N}^{+}$, and $7 \mathbf{a} \cdot \mathbf{E t}_{4} \mathbf{N}^{+}$. Reduced unit cell measurements have been carried out for $1 \mathrm{a} 1 \cdot \mathrm{Me}_{4} \mathrm{~N}^{+}$.

\begin{tabular}{|c|c|c|c|c|}
\hline & $1 \mathrm{a} 1 \cdot \mathrm{Me}_{4} \mathbf{N}^{+}$ & $1 \mathrm{a} 1 \cdot \mathrm{Me}_{4} \mathbf{N}^{+}$ & $1 \mathbf{a} \cdot \mathbf{M e}_{4} \mathbf{N}^{+}$ & $\mathbf{7 a} \cdot \mathbf{E t}_{\mathbf{4}} \mathbf{N}^{+}$ \\
\hline Formula & $\mathrm{C}_{26} \mathrm{H}_{13} \mathrm{~F}_{30} \mathrm{NO}_{2}$ & $\mathrm{C}_{26} \mathrm{H}_{13} \mathrm{~F}_{30} \mathrm{NO}_{2}$ & $\mathrm{C}_{15} \mathrm{H}_{12} \mathrm{~F}_{15} \mathrm{NO}$ & $\mathrm{C}_{22} \mathrm{H}_{20} \mathrm{~F}_{15} \mathrm{~N}_{3}$ \\
\hline Molecule mass & 941.37 & 941.37 & 507.26 & 611.41 \\
\hline Temperature, K & 293 & $173(2)$ & $173(2)$ & $173(2)$ \\
\hline$\lambda\left(\mathrm{MoK}_{\alpha}\right), \AA$ & 0.71073 & 0.71073 & 0.71073 & 0.71073 \\
\hline Cryst syst & Triclinic & & Monoclinic & Monoclinic \\
\hline Cryst size, $\mathrm{mm}^{3}$ & $0.50 \times 0.37 \times 0.28$ & & $1 \times 0.5 \times 0.5$ & $1 \times 0.9 \times 0.4$ \\
\hline Space group & $\mathrm{P}-1$ & & $\mathrm{P} 21 / \mathrm{c}$ & $\mathrm{C} 2 / \mathrm{c}$ \\
\hline$a, \AA$ & $9.604(1)$ & $9.4699(19)$ & $19.476(6)$ & $25.148(4)$ \\
\hline$b, \AA$ & $10.156(2)$ & $10.1381(41)$ & $19.736(3)$ & $11.8370(10)$ \\
\hline$c, \AA$ & $18.032(2)$ & $17.9988(51)$ & $9.657(2)$ & $18.256(2)$ \\
\hline$\alpha,{ }^{\circ}$ & $77.30(1)$ & $77.119(24)$ & 90.00 & 90 \\
\hline$\beta,{ }^{\circ}$ & $76.21(1)$ & $76.119(24)$ & $92.12(2)$ & $111.180(10)$ \\
\hline$\gamma,{ }^{\circ}$ & $77.48(1)$ & $77.257(23)$ & 90.00 & 90 \\
\hline$V, \AA^{3}$ & $1641.1(4)$ & $1607.32(84)$ & $3709.4(15)$ & $5067.3(11)$ \\
\hline$Z$ & 2 & & 8 & 8 \\
\hline$\rho_{\text {calcd }}, \mathrm{g} / \mathrm{cm}^{3}$ & 1.905 & & 1.817 & 1.603 \\
\hline Absorption coefficient $\mu, \mathrm{mm}^{-1}$ & 0.236 & & 0.217 & 0.173 \\
\hline $\mathrm{F}(000)$ & 924 & & 2016 & 2464 \\
\hline$\theta$ range, ${ }^{\circ}$ & $1.18-24.97$ & & $2.54-27.50$ & $2.82-27.5$ \\
\hline Index ranges & $0 \leq h \leq 11$ & & $-25 \leq h \leq 25$ & $-31 \leq h \leq 31$ \\
\hline & $-11 \leq k \leq 12$ & & $-1 \leq k \leq 25$ & $-1 \leq k \leq 14$ \\
\hline & $-20 \leq l \leq 21$ & & $-1 \leq l \leq 12$ & $-23 \leq l \leq 23$ \\
\hline Reflections collected & 6253 & & 10682 & 12484 \\
\hline Absorption correction & PSI-scan & & None & None \\
\hline Completeness to $\theta_{\max }=24.97$ & $99.8 \%$ & & $99.4 \%$ & $98.6 \%$ \\
\hline Independent reflections $/ R_{\text {int }}$ & $5760 / 0.0157$ & & $8487 / 0.0235$ & $5742 / 0.0272$ \\
\hline GOOF of $F^{2}$ & 1.006 & & 1.047 & 1.027 \\
\hline Refinement Method & $\begin{array}{l}\text { full-matrix least- } \\
\text { squares on } \mathrm{F}^{2}\end{array}$ & & $\begin{array}{l}\text { full-matrix } \\
\text { least-squares on } \\
\mathrm{F}^{2}\end{array}$ & $\begin{array}{l}\text { full-matrix } \\
\text { least-squares on } \\
\mathrm{F}^{2}\end{array}$ \\
\hline Data/parameters/restraints & $5760 / 537 / 0$ & & $8487 / 790 / 1056$ & $5742 / 424 / 24$ \\
\hline Final $R$ indices $[I>2 \sigma(I)], R_{1}, w R_{2}$ & $0.0461,0.1202$ & & $0.0806,0.1996$ & $0.0372,0.0939$ \\
\hline$R$ indices (all data), $R_{1}, w R_{2}$ & $0.0784,0.1414$ & & $0.1197,0.2241$ & $0.0500,0.1015$ \\
\hline Extinction coeff, $(\Delta / \sigma)_{\max }$ & $0.0080(12)$ & & $0.0023(5)$ & $0.00091(13)$ \\
\hline Largest diff peak/hole, e/ $/ \AA^{3}$ & $0.302 /-0.244$ & & $0.674 /-0.643$ & $0.363 /-0.238$ \\
\hline $\begin{array}{l}\text { Software for crystallographic } \\
\text { calculations }\end{array}$ & SHELXS-86 ${ }^{11}$ & & \multicolumn{2}{|c|}{ SHELXL-97 ${ }^{12}$} \\
\hline Diffractometer & $\begin{array}{l}\text { Enraf-Nonius } \\
\text { CAD4 }\end{array}$ & \multicolumn{3}{|c|}{ Siemens P4 } \\
\hline
\end{tabular}




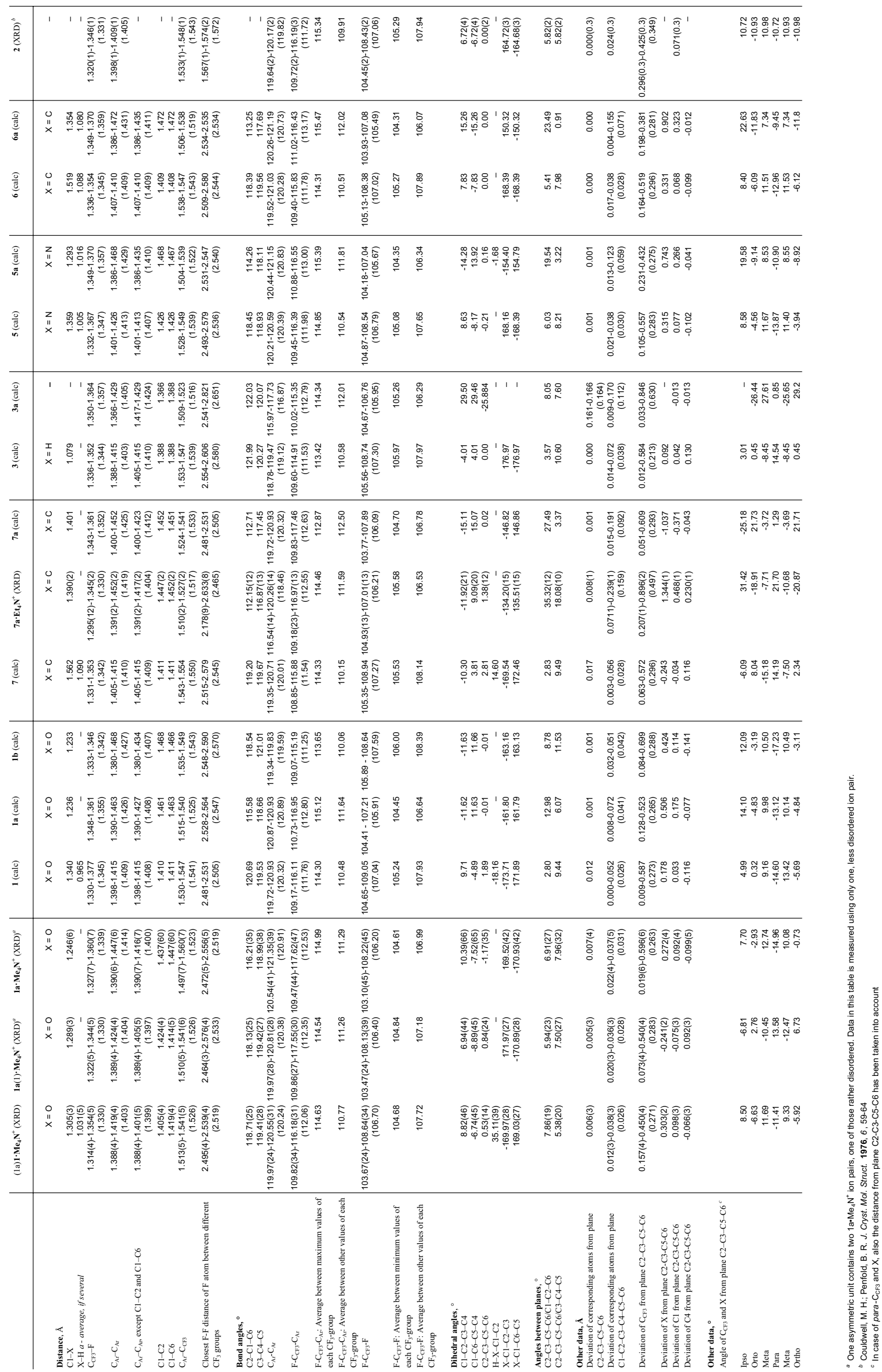




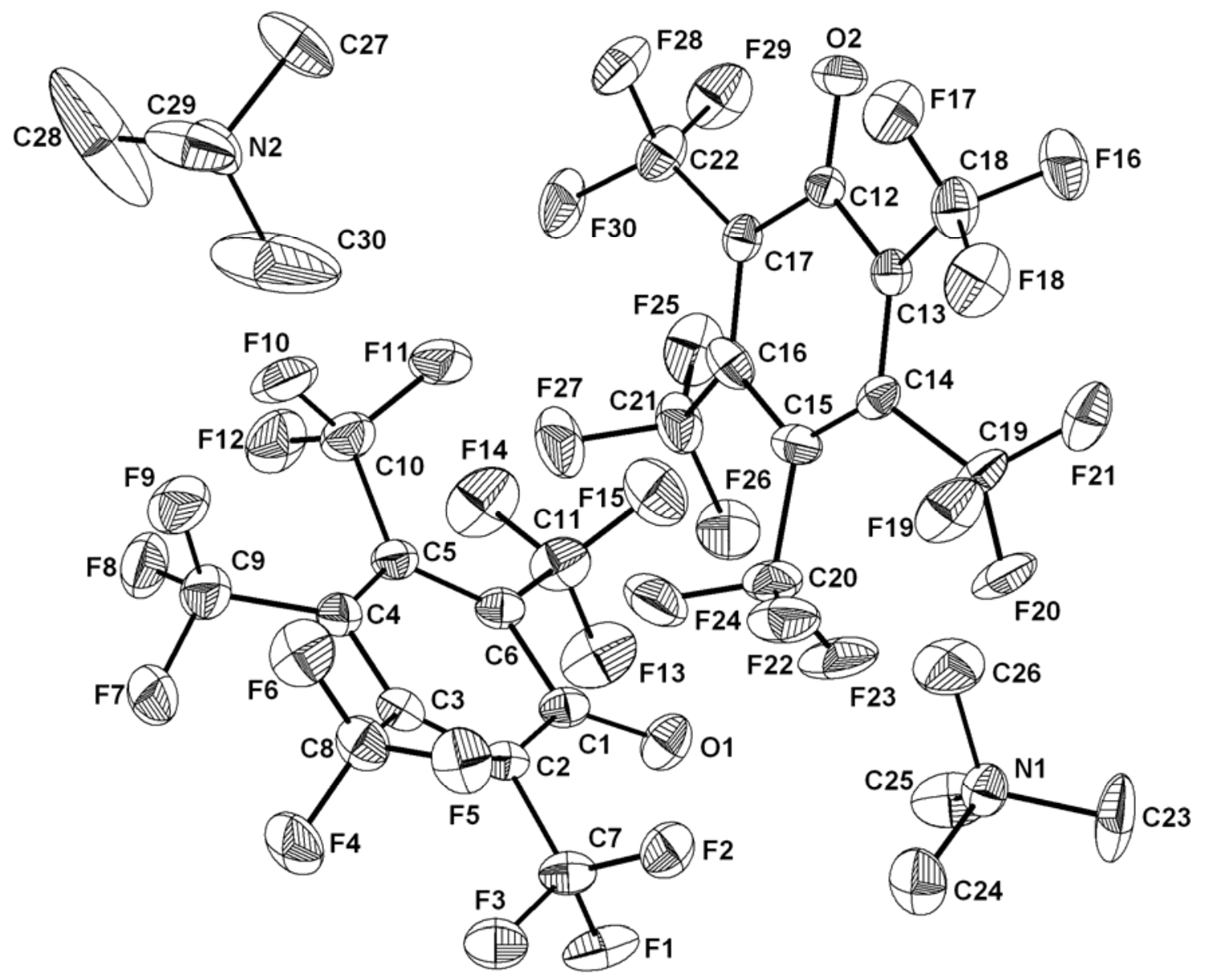

Figure S3. Molecular structure of $1 \mathrm{a} \cdot \mathrm{Me}_{4} \mathrm{~N}^{+}$with $40 \%$ thermal ellipsoids (hydrogen atoms of two $\mathrm{Me}_{4} \mathrm{~N}^{+}$cations are omitted for clarity). One asymmetric unit contains two ion pairs. Both $\mathrm{Me}_{4} \mathrm{~N}^{+}$cations and one $1 \mathrm{a}$ anion were disordered. 

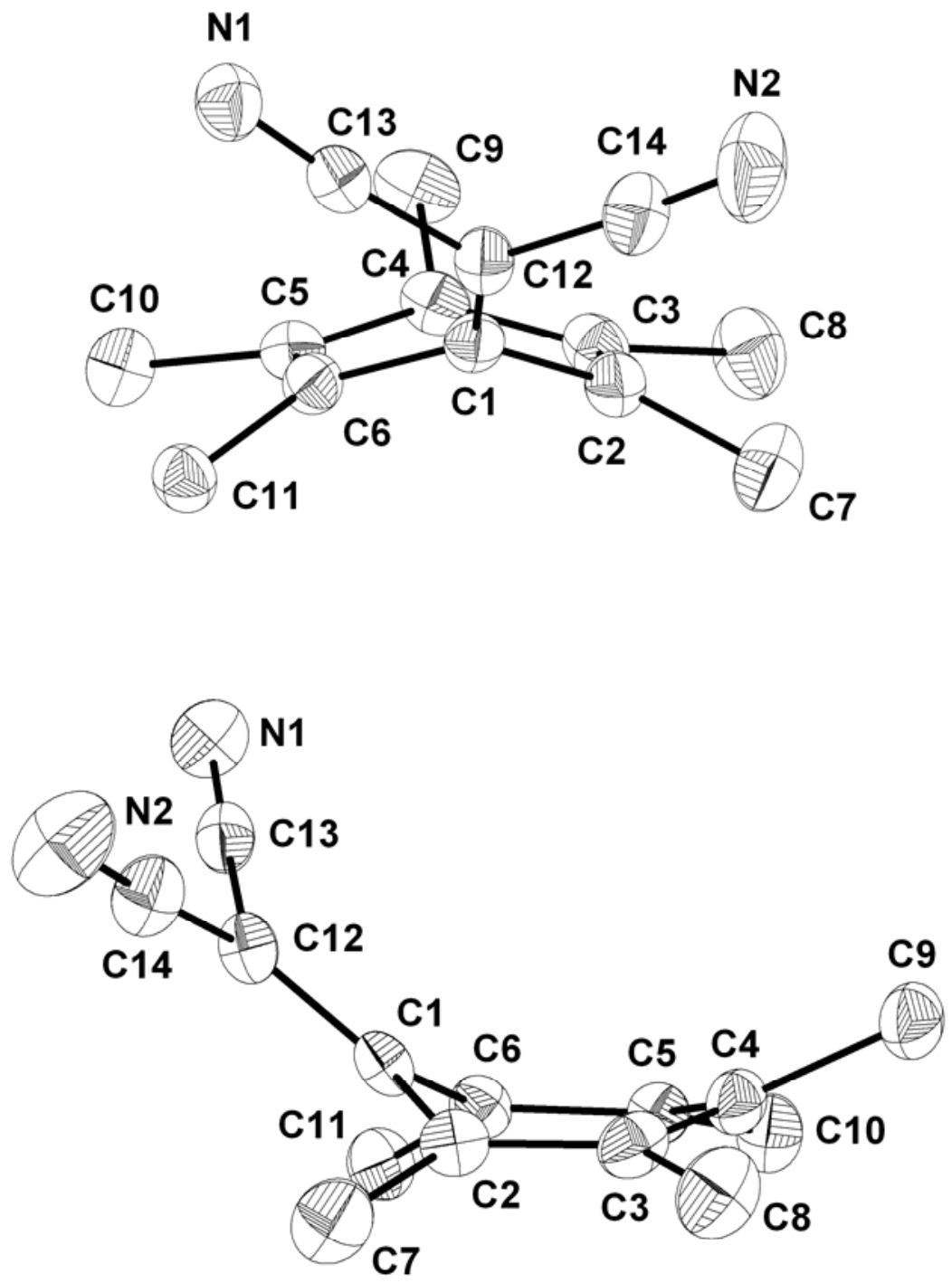

Figure S3. Molecular structure of $7 \mathbf{a} \cdot \mathbf{E t}_{4} \mathbf{N}^{+}$with $50 \%$ thermal ellipsoids to demonstrate non-planarity of the aromatic ring and out of plane positions of the $\mathrm{CF}_{3}$ groups $\left(\mathrm{Et}_{4} \mathrm{~N}^{+}\right.$cation and all fluorine atoms are omitted for clarity). 


\section{Voltammetric Measurements with 1 and 1a}<smiles>Oc1c(C(F)(F)F)c(C(F)(F)F)c(C(F)(F)F)c(C(F)(F)F)c1C(F)(F)F</smiles>

$\mathrm{C}_{11} \mathrm{HF}_{15} \mathrm{O}$

Exact Mass: 433.979

Mol. Wt.: 434.101

$$
\mathrm{C}=1.89 \mathrm{mM}
$$

Solvent: anhydrous $\mathrm{CH}_{3} \mathrm{CN}$

Supporting electrolyte: $\mathrm{NBu}_{4} \mathrm{PF}_{6} 0.1 \mathrm{M}$

Working electrode: Glassy carbon electrode

Reference electrode: saturated calomel electrode (SCE)

The compound shows an oxidation potential at $+1.66 \mathrm{~V}$ vs $\mathrm{SCE}\left(\mathrm{Ep}_{\mathrm{ox}}\right.$, peak potential at $0.2 \mathrm{~V} / \mathrm{s})$. On the reverse scan there is a reduction step at $+1.44 \mathrm{~V} v s \mathrm{SCE}\left(\mathrm{Ep}_{\text {red }}\right) . \Delta \mathrm{Ep}$ is close to $220 \mathrm{mV}$. It is a quasi-reversible system, demonstrating that the radical cation is relatively stable (Figure $\mathrm{S} 4)$. Potential standard $\mathrm{E}^{0}=\left(E \mathrm{p}_{\mathrm{ox}}+\mathrm{E} \mathrm{p}_{\mathrm{red}} / 2\right)$ is equal to $+1.55 \mathrm{~V}$ vs SCE.

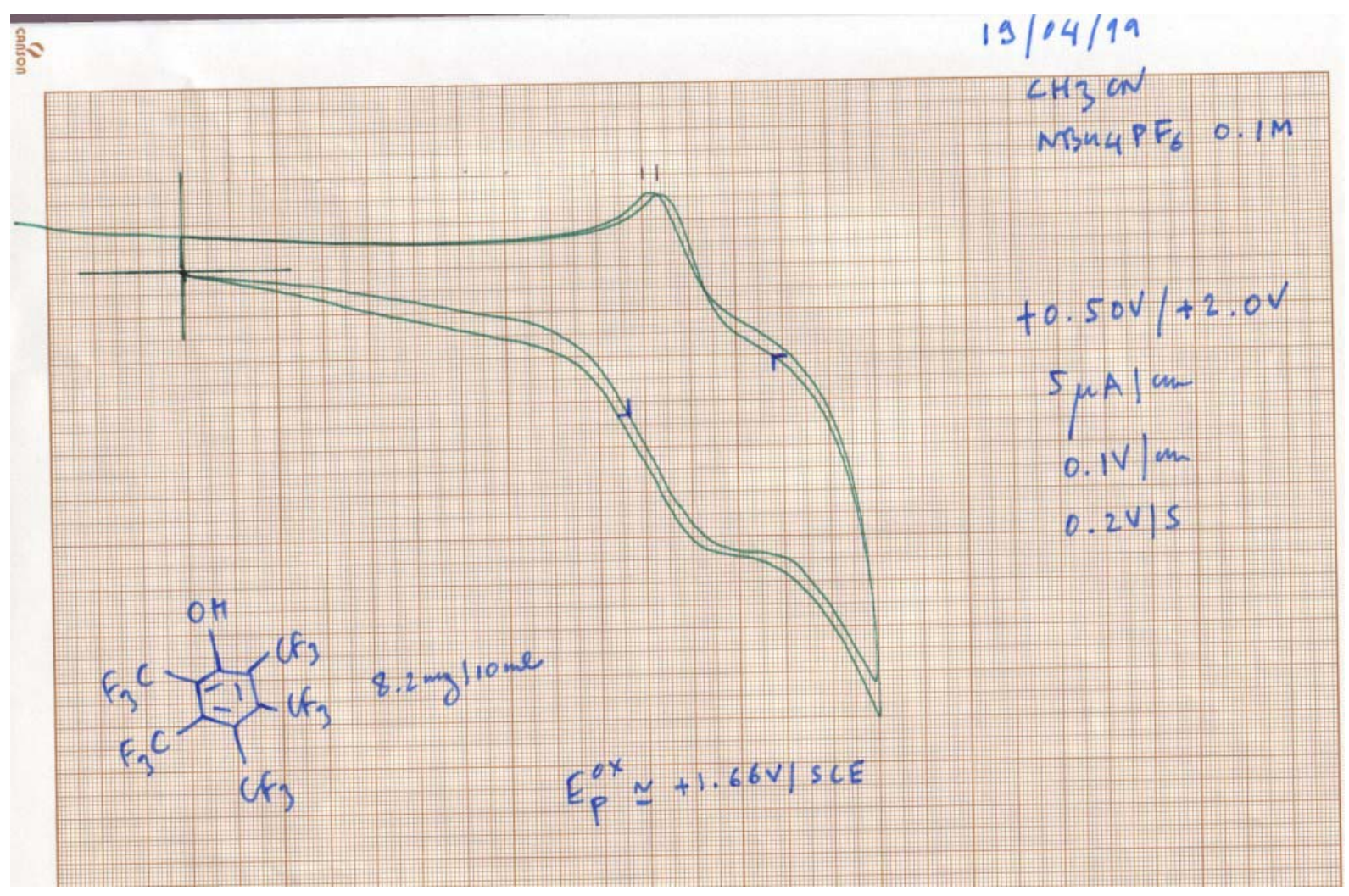

Figure S4. Cyclic voltammetry of $1 ; C=1.89 \mathrm{mM}^{\text {in }} \mathrm{CH}_{3} \mathrm{CN}+0.1 \mathrm{M} \mathrm{NBu}_{4} \mathrm{PF}_{6}$. Scan rate $=0.2 \mathrm{~V} / \mathrm{s}$. Potential scan: $+0.5 \mathrm{~V}$ to $+2.0 \mathrm{~V}$. 
The compound is reducible at a potential close to $-1.1 \mathrm{~V} v s$ SCE (peak potential at 0.2 $\mathrm{V} / \mathrm{s})$; it is an irreversible reduction step demonstrating that the radical-anion is unstable (Figure S5).

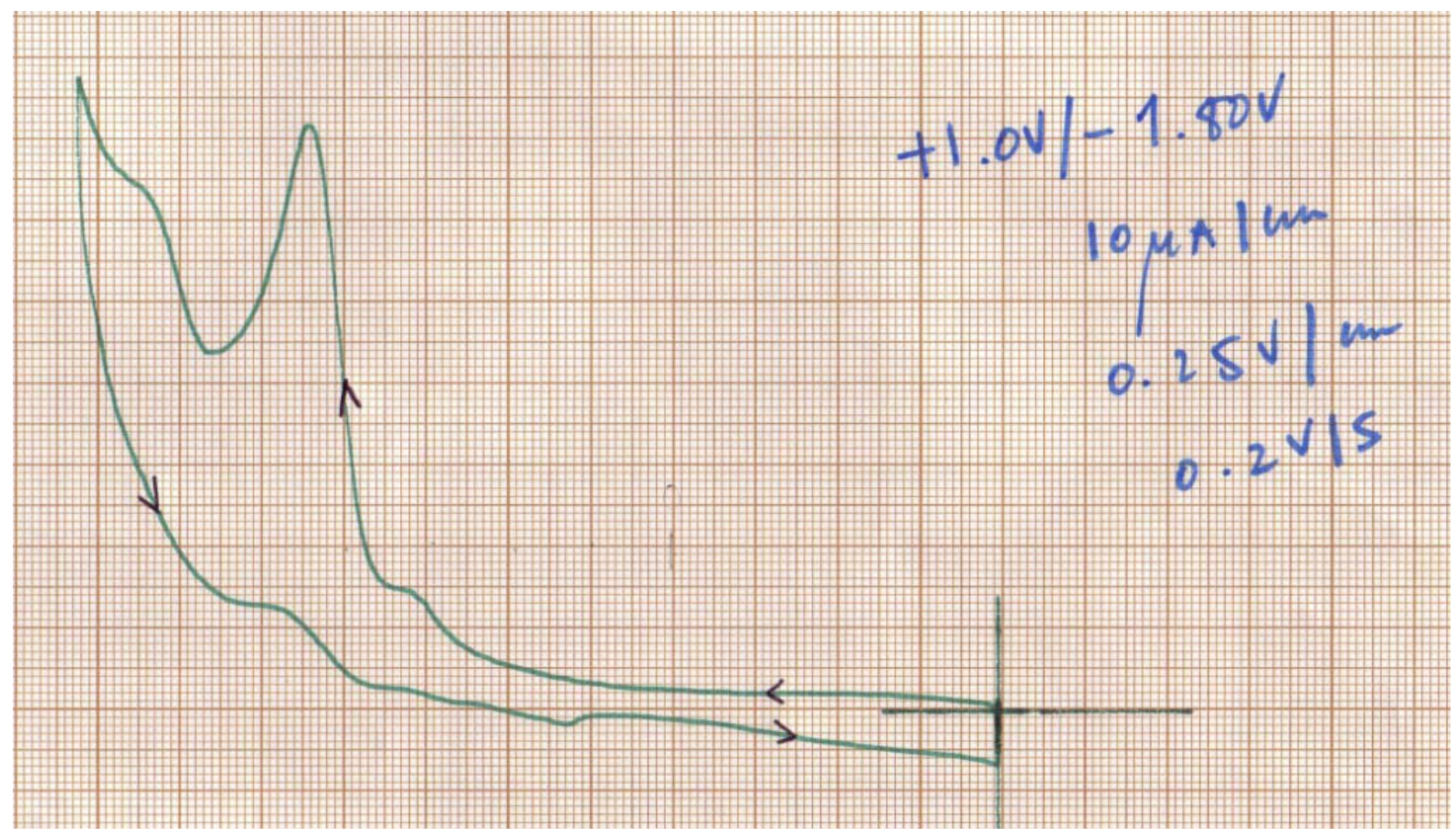

Figure S5. Cyclic voltammetry of $1 ; C=1.89 \mathrm{mM}^{\text {in }} \mathrm{CH}_{3} \mathrm{CN}+0.1 \mathrm{M} \mathrm{NBu}_{4} \mathrm{PF}_{6}$. Scan rate $=0.2 \mathrm{~V} / \mathrm{s}$. Potential scan: $+1.0 \mathrm{~V}$ to $-1.8 \mathrm{~V}$ 
<smiles>COc1c(C(F)(F)F)c(C(F)(F)F)c(C(F)(F)F)c(C(F)(F)F)c1C(F)(F)F</smiles>

$\mathrm{C}_{15} \mathrm{H}_{12} \mathrm{~F}_{15} \mathrm{NO}$

Exact Mass: 507.068

Mol. Wt.: 507.238

$$
\mathrm{C}=2.37 \mathrm{mM}
$$

Solvent: anhydrous $\mathrm{CH}_{3} \mathrm{CN}$

Supporting electrolyte: $\mathrm{NBu}_{4} \mathrm{PF}_{6} 0.1 \mathrm{M}$

Working electrode: Glassy carbon electrode

Reference electrode: saturated calomel electrode (SCE)

The compound shows an oxidation potential at $+1.52 \mathrm{~V}$ vs SCE $\left(\mathrm{Ep}_{\mathrm{ox}}\right.$, peak potential at $0.2 \mathrm{~V} / \mathrm{s})$. On the reverse scan there is a reduction step at $+1.45 \mathrm{~V} v s \mathrm{SCE}\left(\mathrm{Ep}_{\mathrm{red}}\right) . \Delta \mathrm{Ep}$ is close to $75 \mathrm{mV}$. It is a quasi-reversible system, demonstrating that the oxidation of the phenoxide gives a quite stable species, probably the phenoxy radical (Figure S6). Potential standard $\mathrm{E}^{0}=\left(\mathrm{Ep}_{\mathrm{ox}}+\mathrm{Ep}_{\mathrm{red}} / 2\right)$ is equal to $+1.485 \mathrm{~V} v \mathrm{SCE}$.

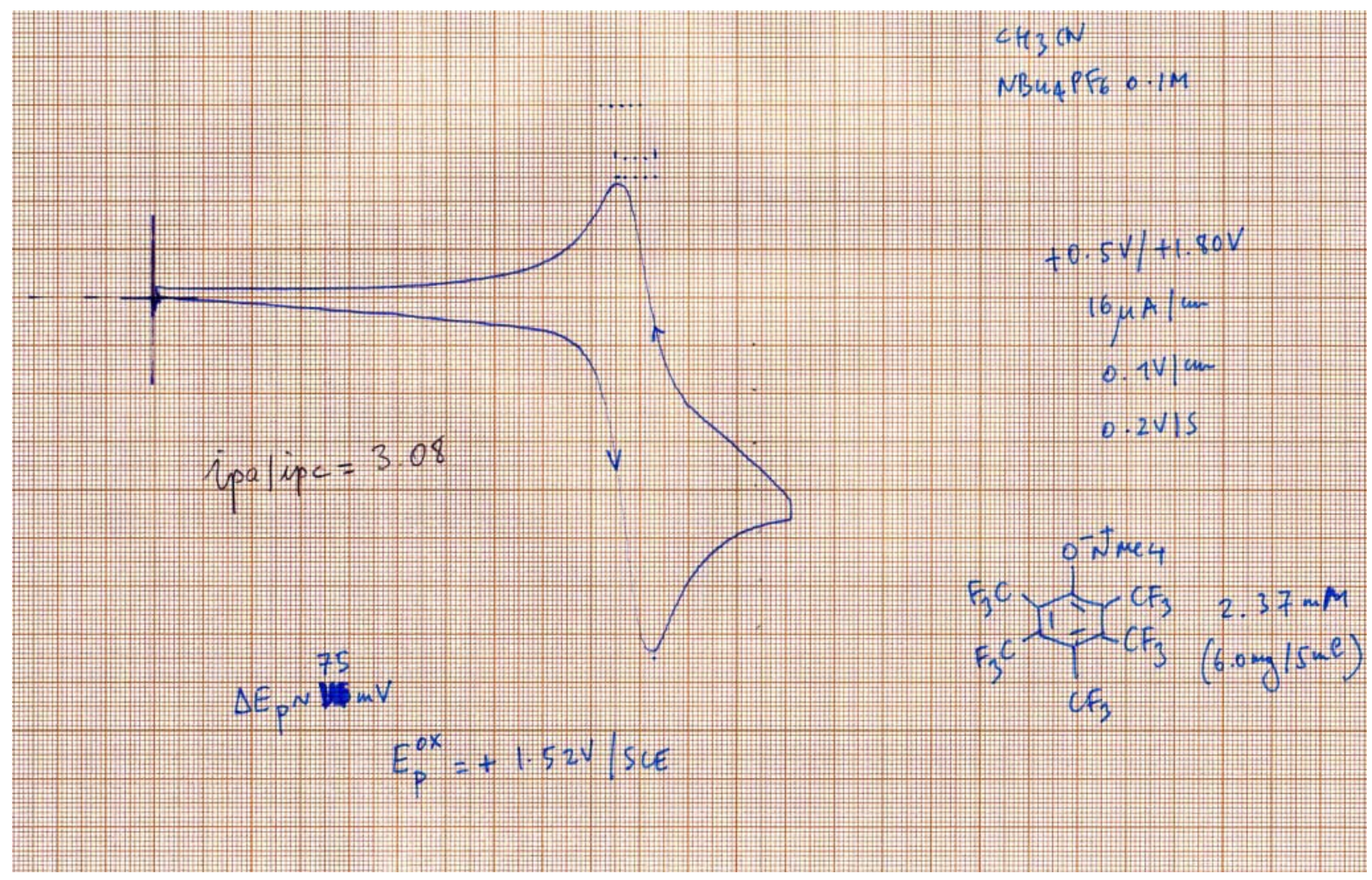

Figure S6. Cyclic voltammetry of $1 \mathrm{a} \cdot \mathrm{Me}_{4} \mathrm{~N}^{+} ; C=2.37 \mathrm{mM}$ in $\mathrm{CH}_{3} \mathrm{CN}+0.1 \mathrm{M}$ $\mathrm{NBu}_{4} \mathrm{PF}_{6}$. Scan rate $=0.2 \mathrm{~V} / \mathrm{s}$. Potential scan: $+0.5 \mathrm{~V}$ to $+1.8 \mathrm{~V}$ 
The compound is also reducible at a potential close to $-2.16 \mathrm{~V} v s$ SCE (peak potential at $0.2 \mathrm{~V} / \mathrm{s})$; it is an irreversible reduction step demonstrating that the radical-anion is unstable (Figure S7).

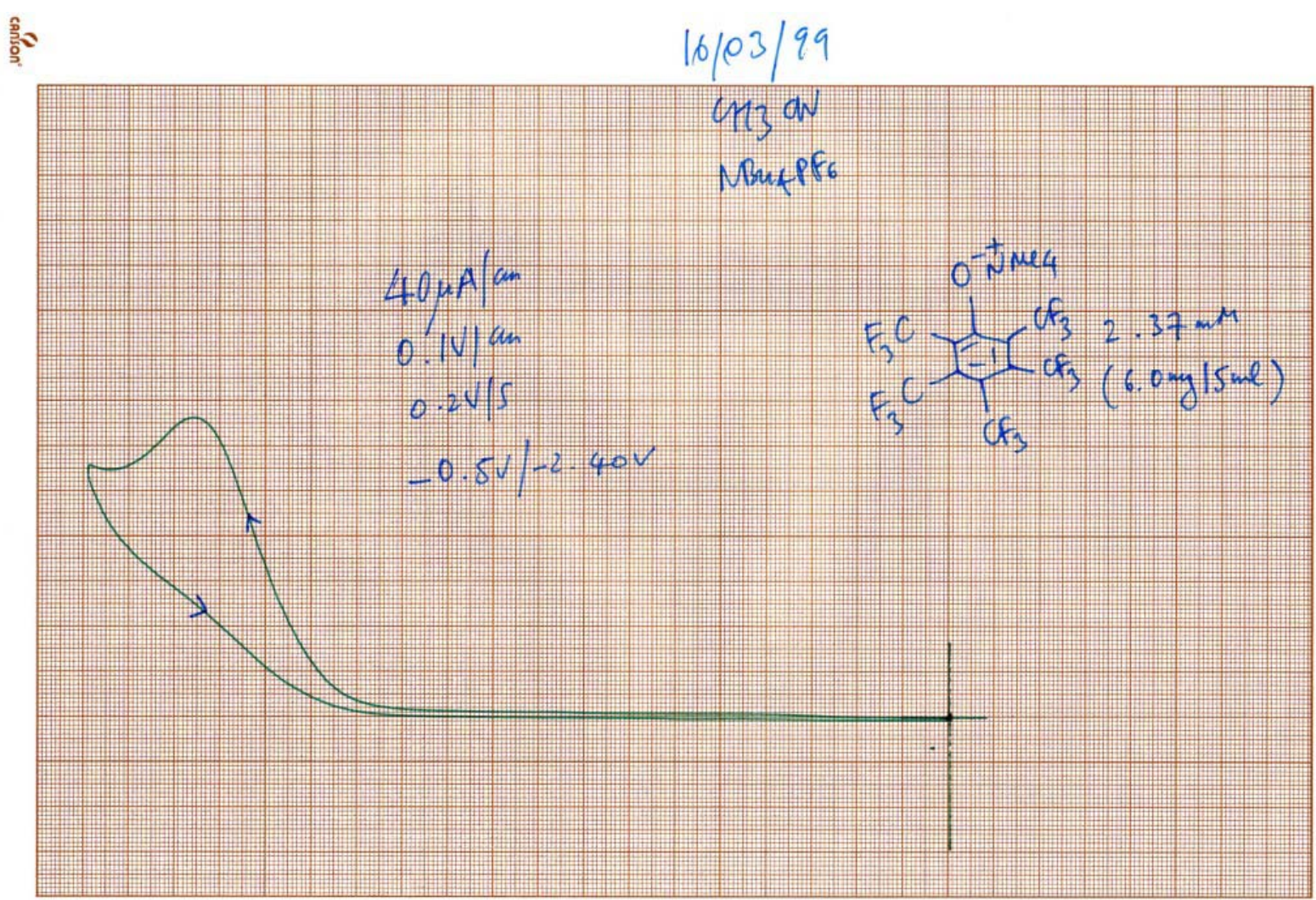

Figure S7. Cyclic voltammetry of $1 \mathrm{a} \cdot \mathrm{Me}_{4} \mathrm{~N}^{+} ; C=2.37 \mathrm{mM}$ in $\mathrm{CH}_{3} \mathrm{CN}+0.1 \mathrm{M}$ $\mathrm{NBu}_{4} \mathrm{PF}_{6}$. Scan rate $=0.2 \mathrm{~V} / \mathrm{s}$. Potential scan: $-0.5 \mathrm{~V}$ to $-2.4 \mathrm{~V}$

Materials and equipment (same as in ref 12):

Working electrode: glassy carbon disc (3 $\mathrm{mm}$ diameter)

Auxiliary electrode: Platinum

Reference electrode: Saturated Calomel Electrode (SCE) Solvent: Anhydrous $\mathrm{CH}_{3} \mathrm{CN}$

Supporting electrolyte: Tetrabutylammonium hexafluorophosphate, $\mathrm{NBu}_{4} \mathrm{PF}_{6} 0.1 \mathrm{M}$.

Under positive nitrogen atmosphere; before any voltamogram was recorded, nitrogen was bubbled into the solution of $\mathrm{CH}_{3} \mathrm{CN} / \mathrm{NBu}_{4} \mathrm{PF}_{6} 0.1 \mathrm{M}$ for at least 15 minutes, in order to remove any trace of oxygen. A voltamogram is then first recorded without any added substrate to check for any electro-active impurities. Then the substrate is added. 


\section{Electrochemical equipment:}

'Home-made potentiostat ${ }^{\text {'13 }}$ with a positive feedback ohmic drop compensation and a signal generator. 


\section{References}

1. Mattern, D. L. J. Org. Chem. 1984, 49, 3051-3053.

2. (a) Yagupolskii, L. M.; L. M.; Popov, V. I.; Kondratenko, N. V. Zh. Org. Chem. 1976, 12, 916-917. (b) Deacon, G. B.; Farquharson, G. J. Aust. J. Chem. 1976, 29, 627-635. (c) Deacon, G. B.; Farquharson, G. J. Aust. J. Chem. 1977, 30, 1701-1713.

3. Stoyanov. E. S.; Hoffman, S. P.; Juhasz, M.; Reed, C. A. J. Am. Chem. Soc. 2006, $128,3160-3161$.

4. Wiemers, D. M.; Burton, D. J. J. Am. Chem. Soc. 1986, 108, 832-834.

5. (a) Willert-Porada, M. A.; Burton, D. J; Baenziger, N. C. J. Chem. Soc. Chem. Commun. 1989, 1633-1634. (b) Burton, D. J. Organometallics in Synthetic Organofluorine Chemistry in: Synthetic Fluorine Chemistry, Wiley, New York, 1992.

6. Kütt, A.; Leito, I.; Kaljurand, I.; Sooväli, L.; Vlasov, V.M.; Yagupolskii, L.M.; Koppel, I.A. J. Org. Chem., 2006, 71, 2829-2838.

7. Bartmess, J. E. Negative Ion Energetics Data in NIST Chemistry WebBook, NIST Standard Reference Database Number 69, Eds. Linstrom P. J. and Mallard, W. G., June 2005, National Institute of Standards and Technology, Gaithersburg MD, 20899 (http://webbook.nist.gov).

8. Koppel, I. A.; Koppel, J.; Leito, I.; Koppel, I.; Mishima, M.; Yagupolskii, L. M. J. Chem. Soc., Perkin Trans. II, 2001, 229-232.

9. Koppel, I. A.; Koppel, J. B.; Pihl, V.; Leito, I.; Mishima, M; Vlasov, V. M.; Yagupolskii, L. M.; Taft, R. W. J. Chem. Soc., Perkin Trans. II, 2000, 1125-1133.

10. Sheldric, G. M. SHELXS-86. Program for the Solution of Crystal Structures, University of Gottingen, Gottingen, Germany, 1986.

11. Sheldric, G. M. SHELXL-97. Program for the Refinement of crystal Structures, University of Gottingen, Gottingen, Germany, 1997. 
12. Kolomeitsev, A. A; Medebielle, M.; Kirsch, P.; Lork, E.; Röschenthaler, G.-V. J. Chem. Soc., Perkin Trans 1, 2000, 2183-2185.

13. Garreau; D; Savéant, J-M. J. Electroanal. Chem. 1972, 35, 309. 


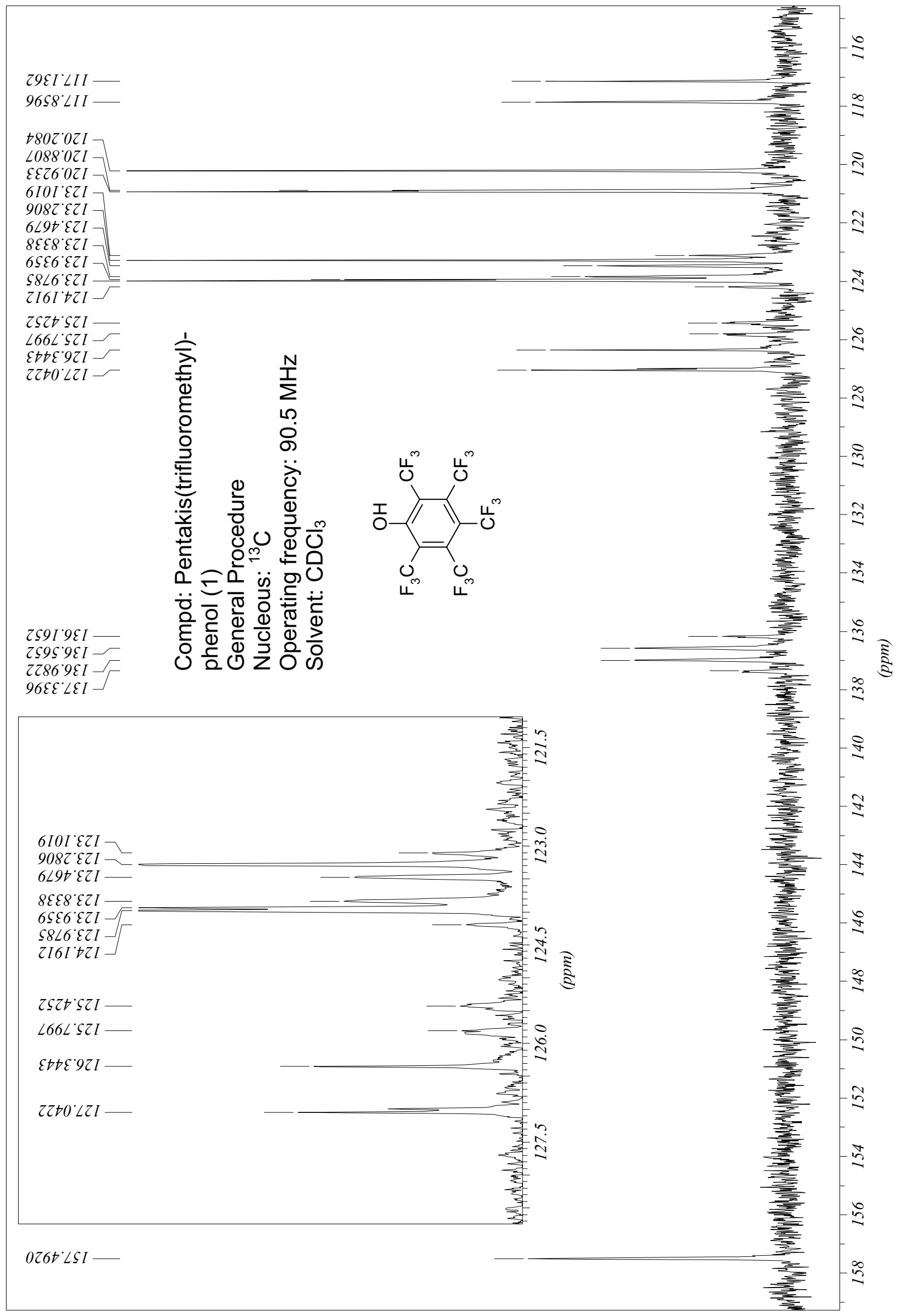


G8'GG-

9L'G -

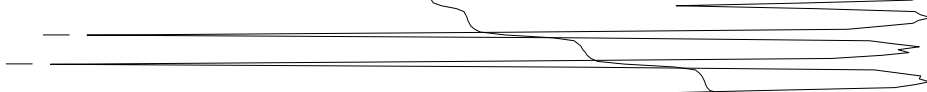

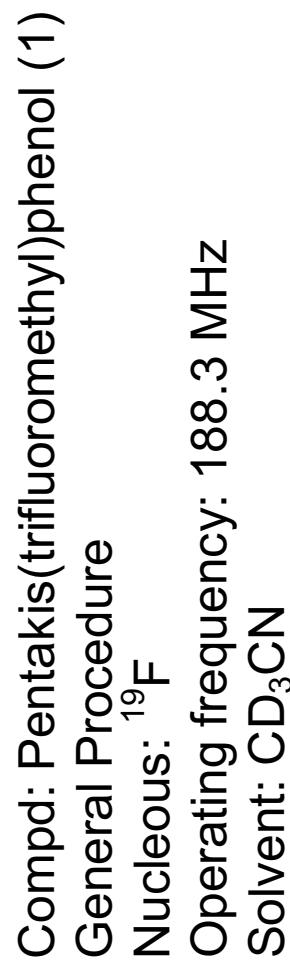

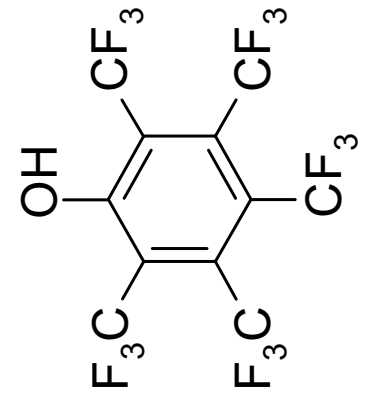

Z9'IS-

EG'LG'

カt'เG'

St'6t-

$9 \varepsilon^{\prime} 6 t^{-}$

$\angle Z^{\prime} 6 \nabla^{-}$

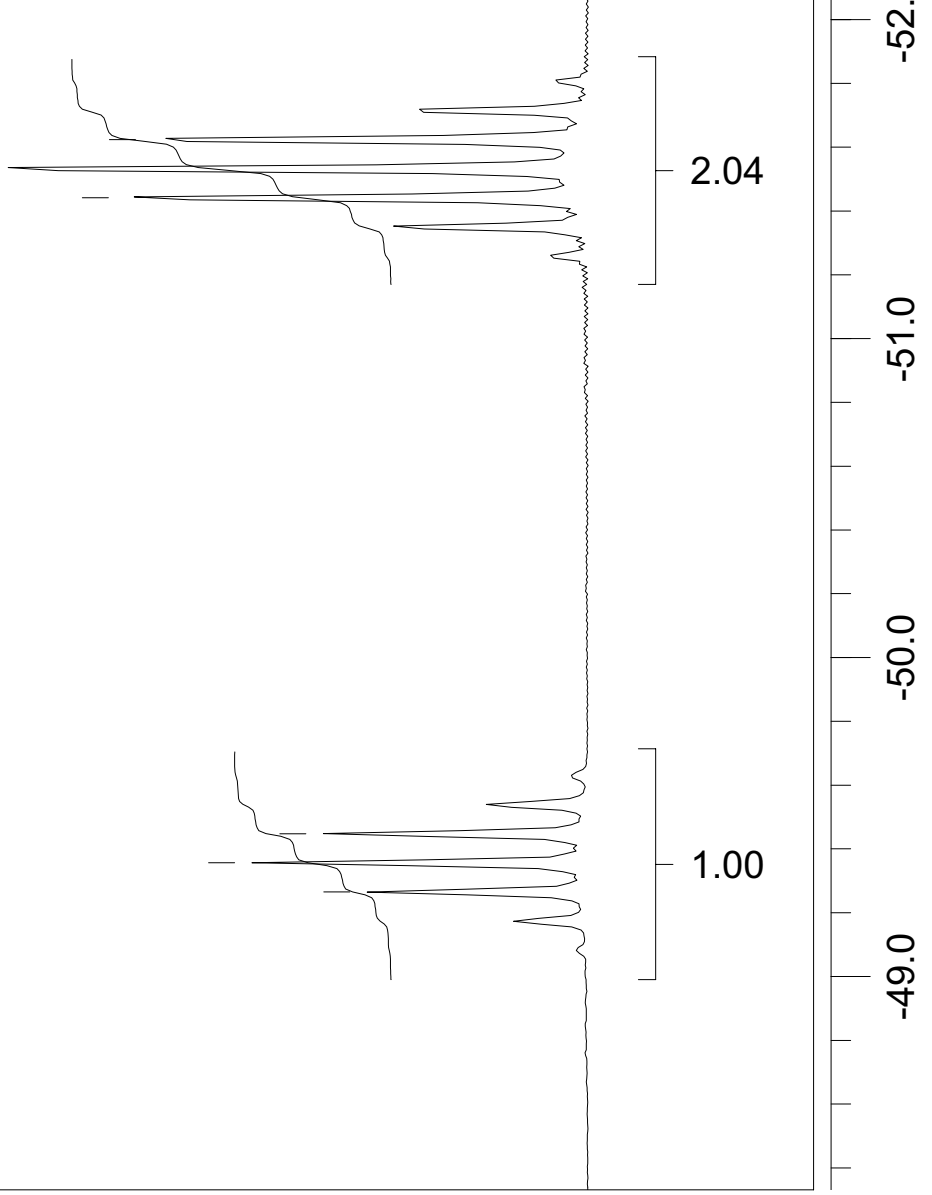

$\underset{\Xi}{E}$
$\frac{\partial}{2}$ 
80'SG-

$86^{\prime} \downarrow \mathrm{G}^{-}$

$68^{\prime} \mathrm{tG}$ -

$6 L^{\prime} \forall G$ -

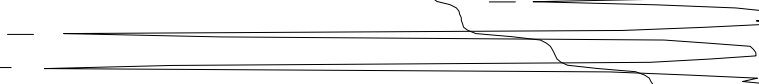

1.71

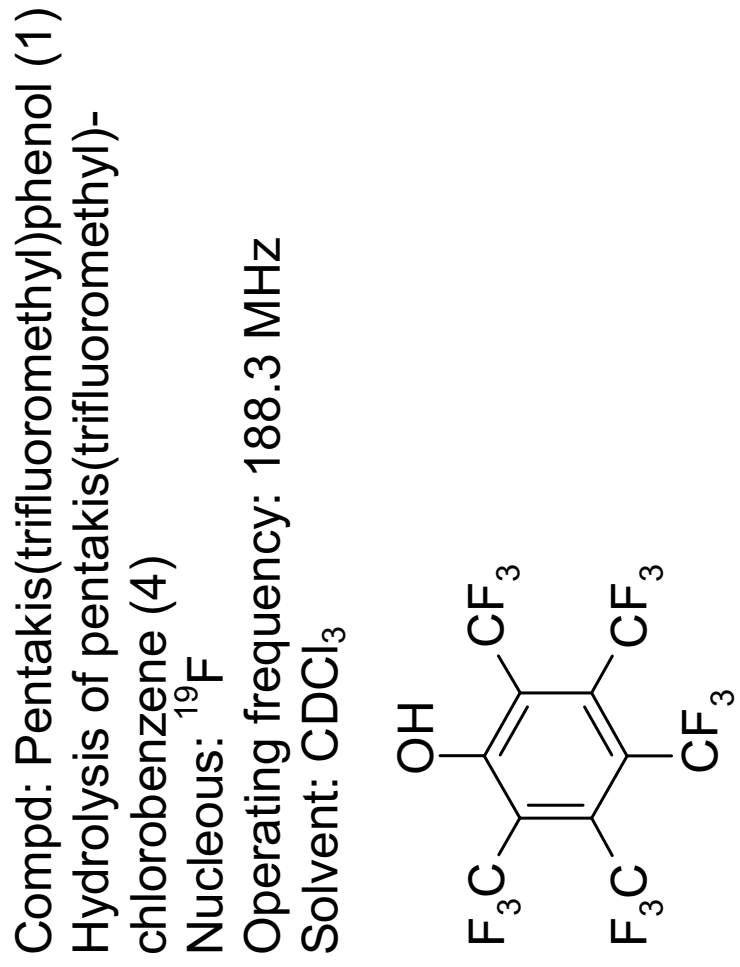

$\nabla L^{\prime} 0 S^{-}$

S9'0G-

SG'OS-

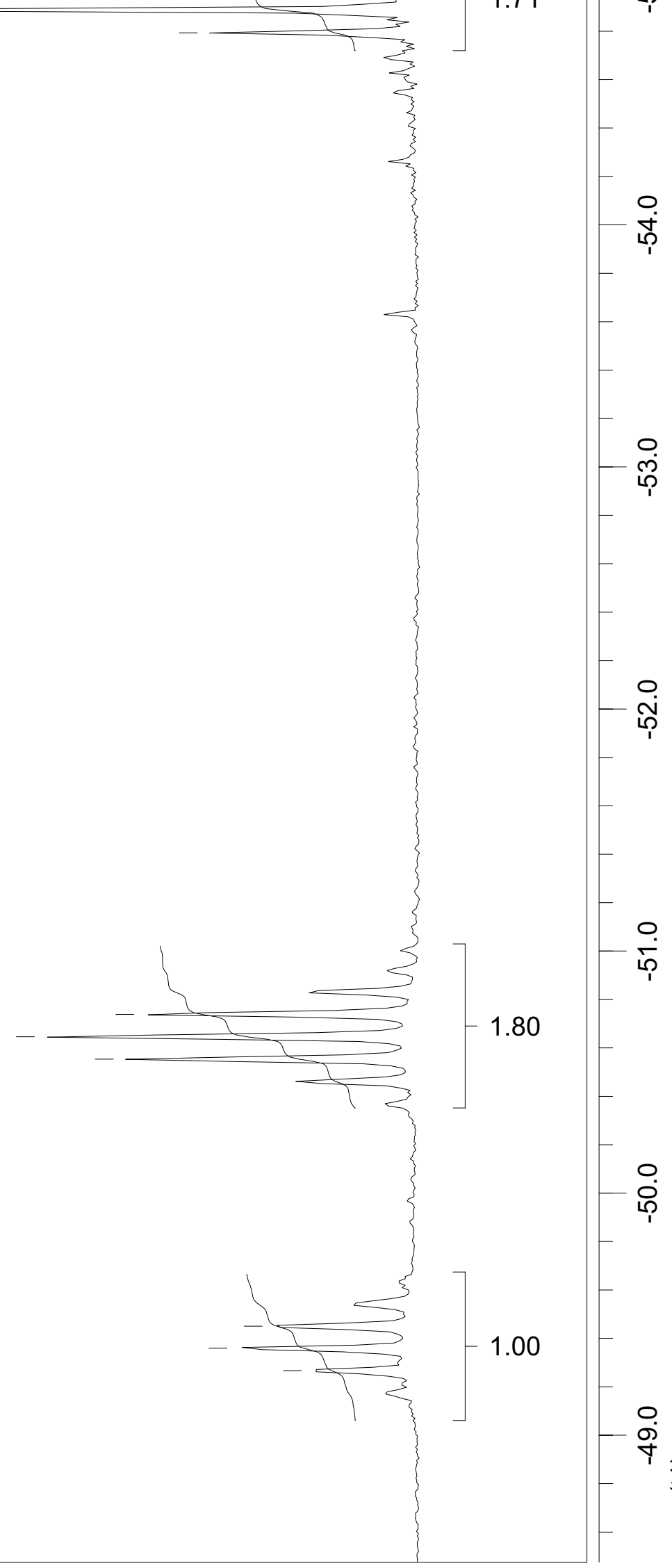

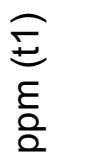




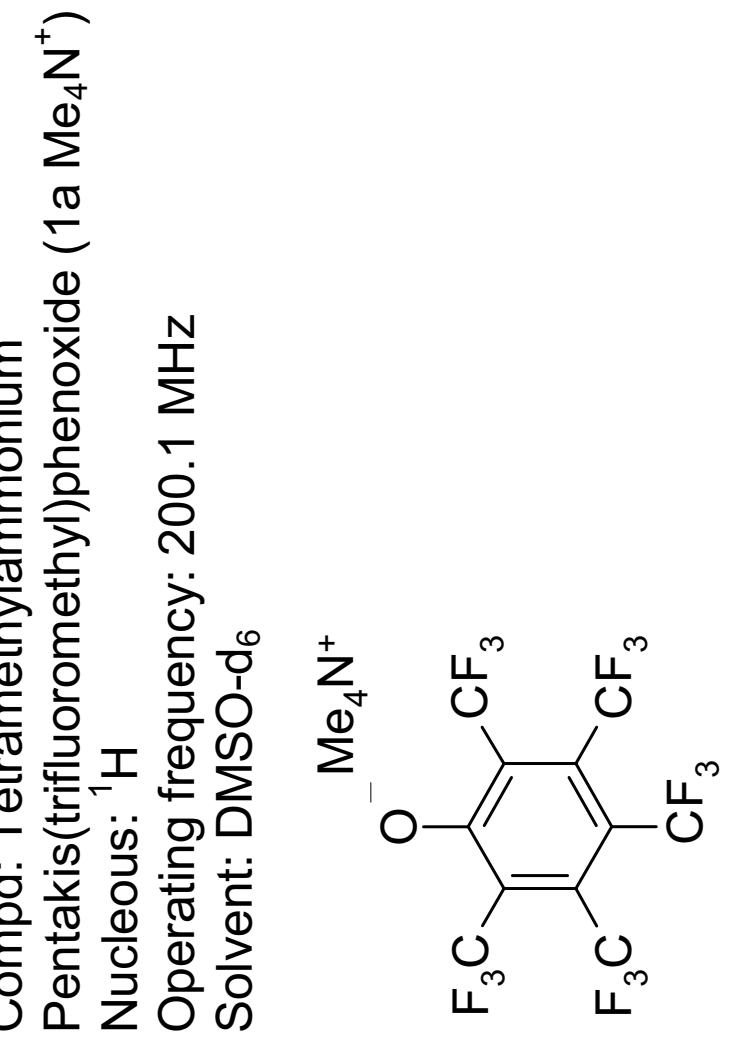

OL $\mathcal{1}$

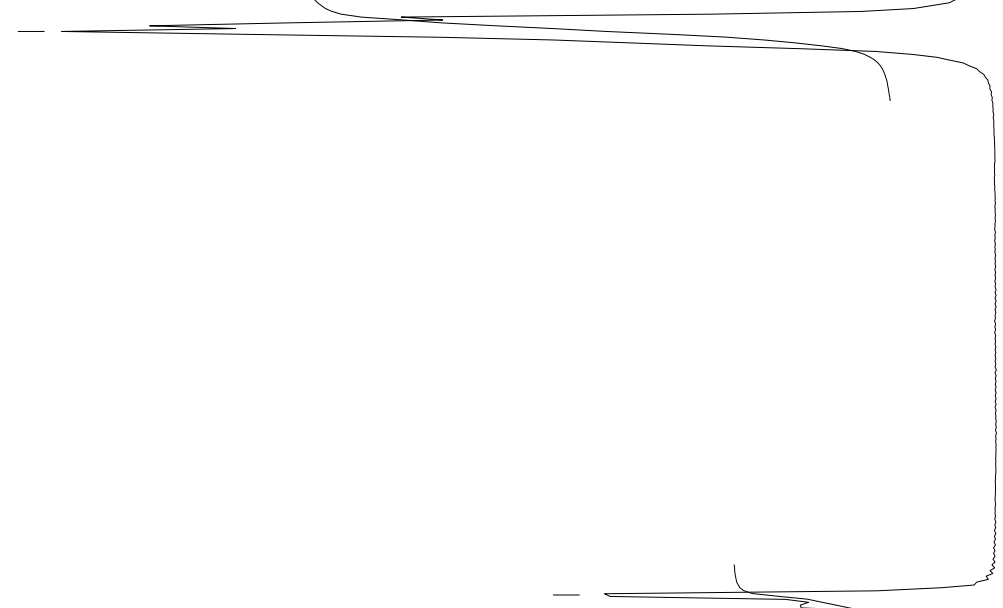


82 89-

61.89-

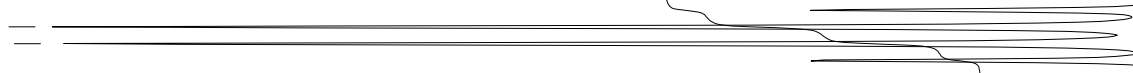

てヤ゙૬-

$\downarrow \varepsilon$ ¿G-

9乙'ZG-
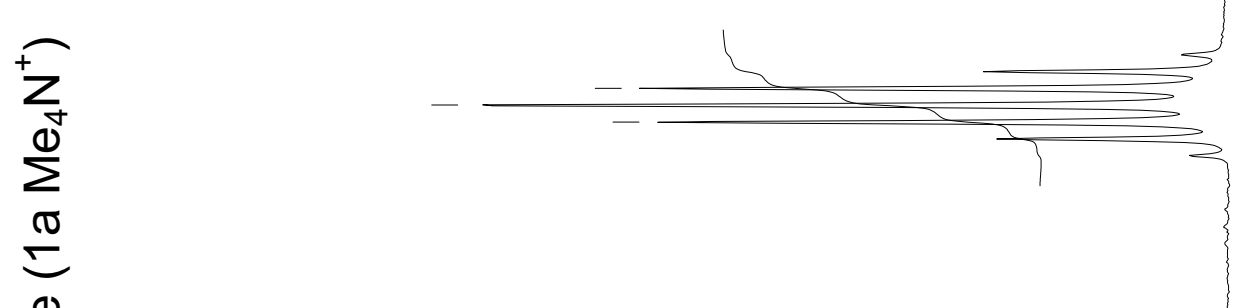

를 을 №

을을

$\frac{\varepsilon}{\bar{\Sigma}} \stackrel{\infty}{\infty} \quad \stackrel{\infty}{\Sigma}$

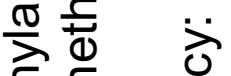

동 잉

$\frac{\varepsilon}{\bar{\sigma}}$ 음

느능 ฮ

过 는은 这

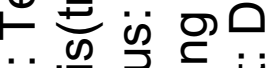

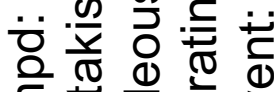

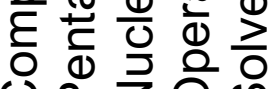

$6 L^{\circ} \angle t$

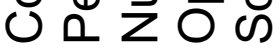

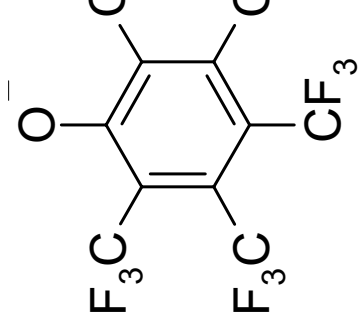

レレ $\angle b$

$\varepsilon 0^{\circ} \angle b^{-}$ 


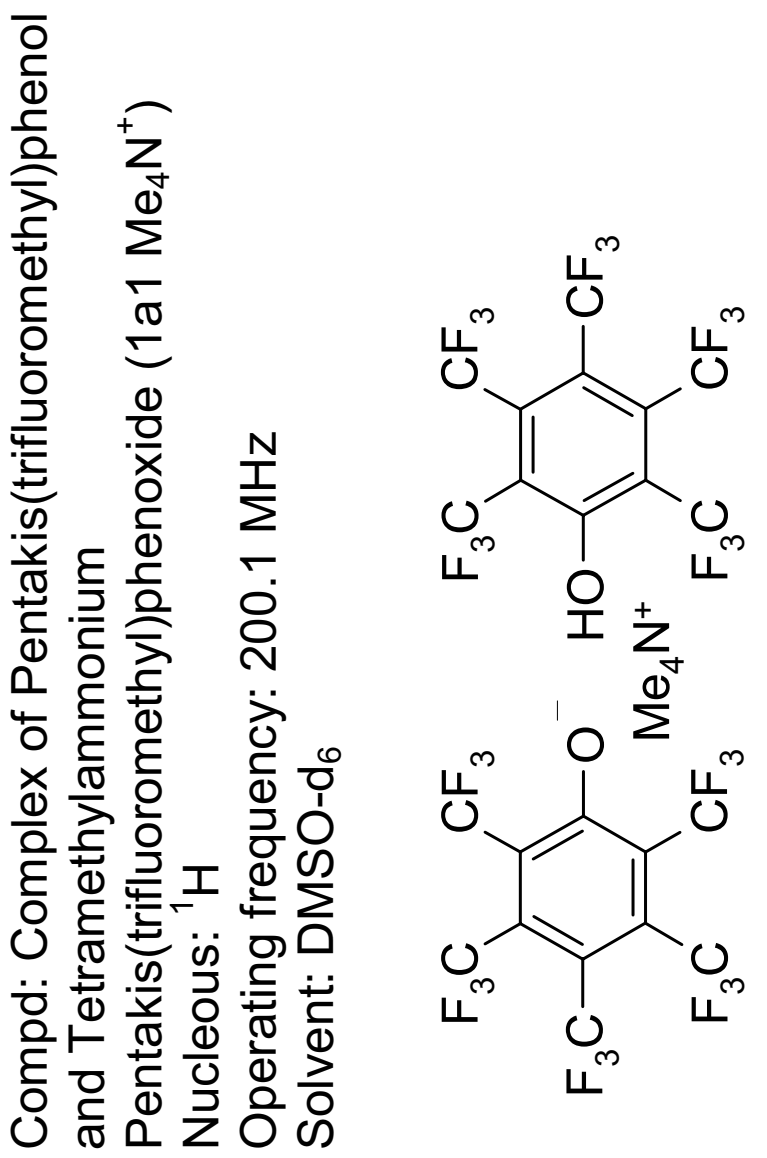

$8 t^{\prime} 2$

$\angle 0^{`} \varepsilon$

$98^{\prime} t$

$\angle 8^{\circ} t$

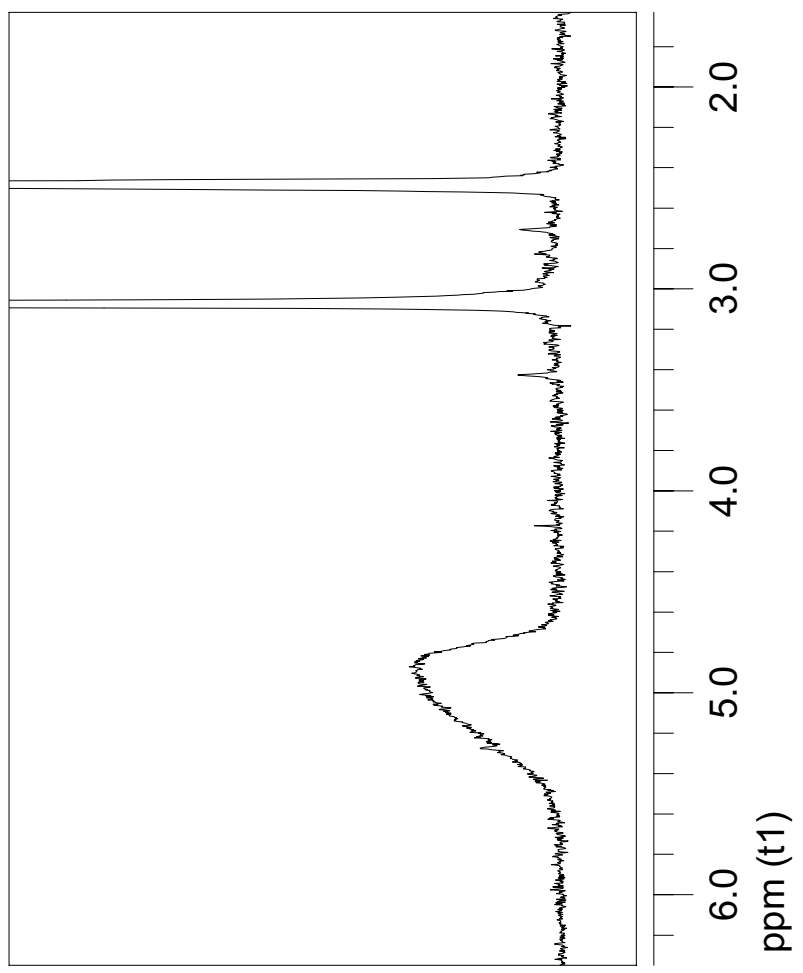

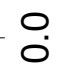


Z2 89 -

까 89

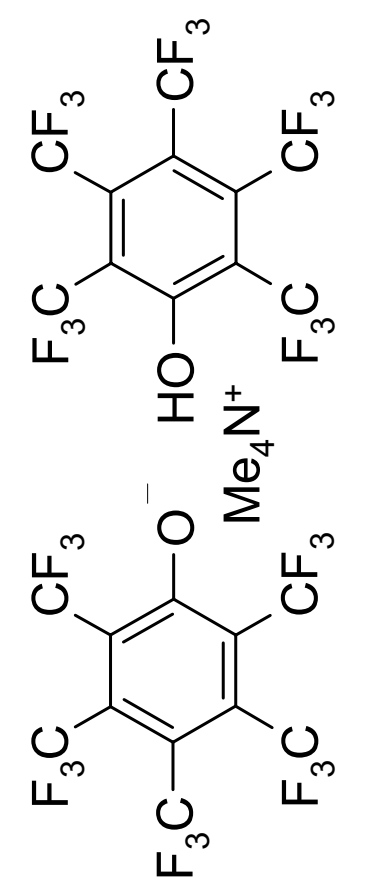

6ย'ZS-

เE'ZG-

乙乙 乙૬-

$0 Z^{\prime} \angle t^{-}$

ルレ $\angle b^{-}$

$\varepsilon 0^{\circ} \angle b^{-}$

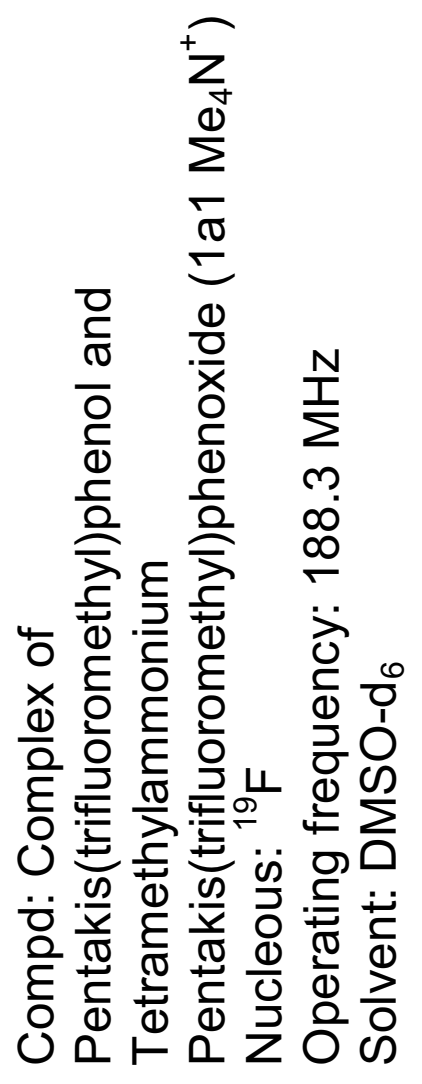


ZL'9L

81 ㄴL

$87^{\circ} 6 \mathrm{~L}$

E6.6 L

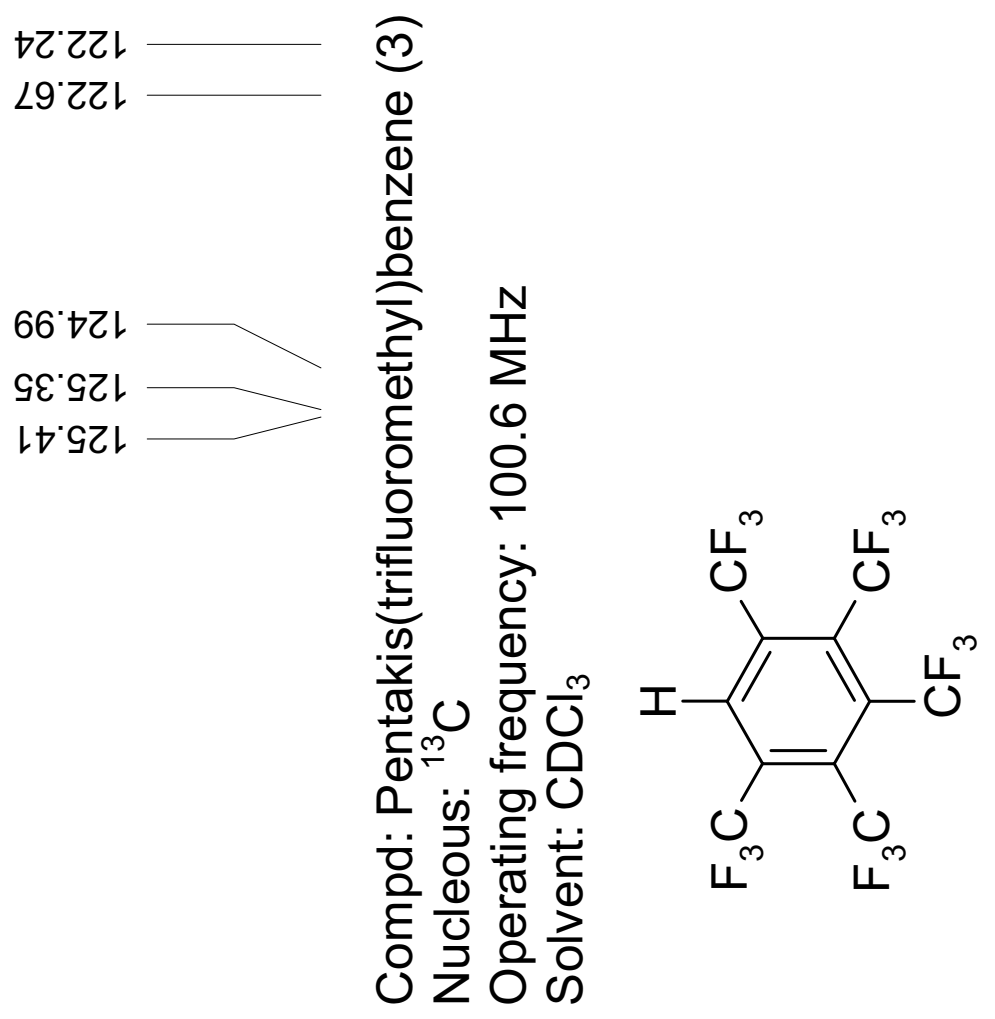

OLIEL

† ${ }^{\circ} \varepsilon \varepsilon \downarrow$

しでレレ

$G \varepsilon^{\circ} \downarrow \varepsilon L$

$0 \angle \forall \varepsilon L$

५o $\varsigma \varepsilon \downarrow$ 
$\nabla \mathcal{G}^{\circ}$

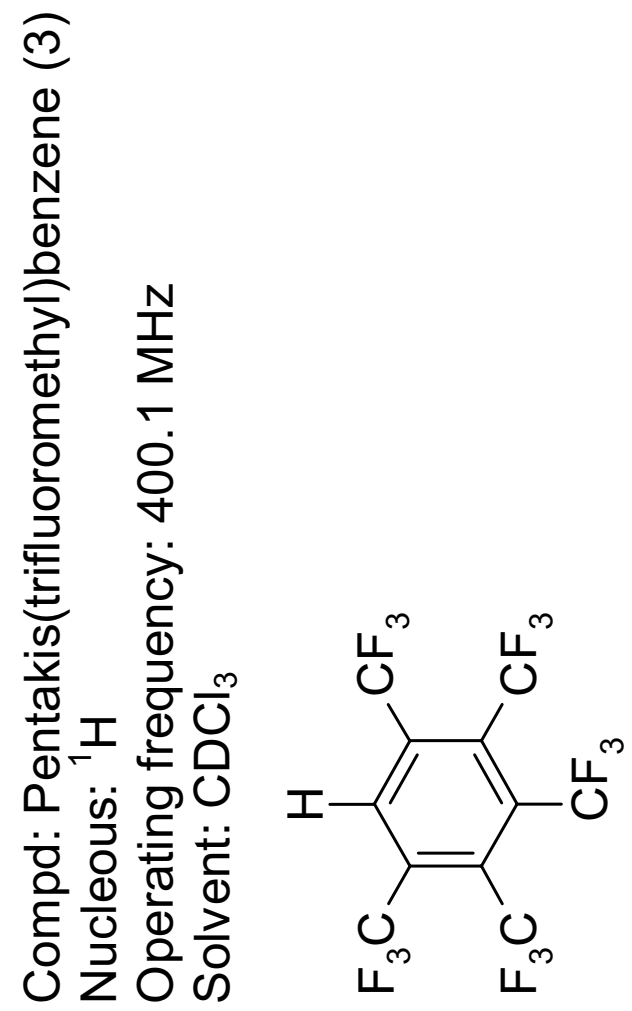

$9 Z^{\prime} L$

19.8 
กี

(

d

ฮ

츨 N

है

임

旁

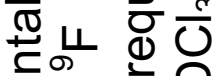

안

$\triangle$ is

远

엉

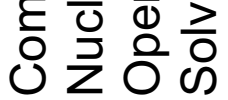

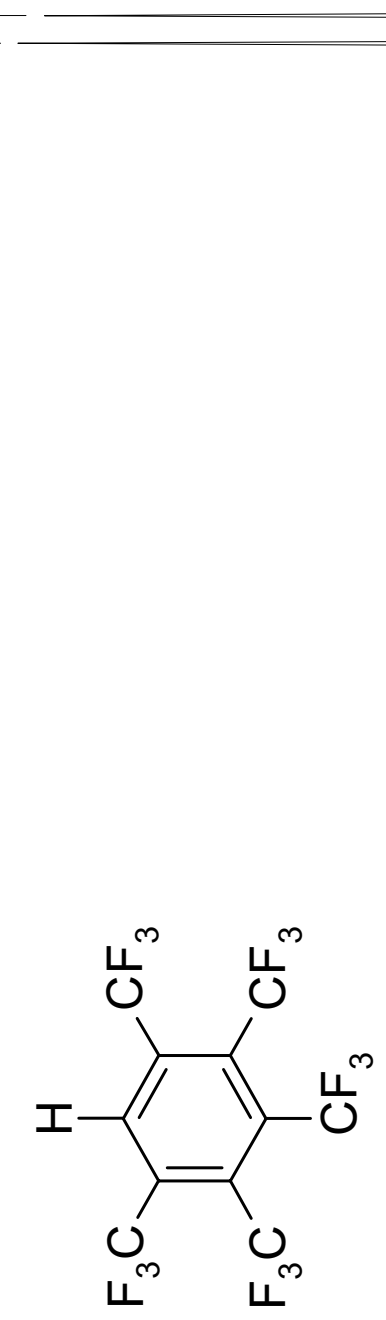

$88^{\circ} \mathrm{tG}-$

$08^{\circ} \nabla G^{-}$

L'tG'

カた'ZG-

५E'ZS-

9乙'ZG-
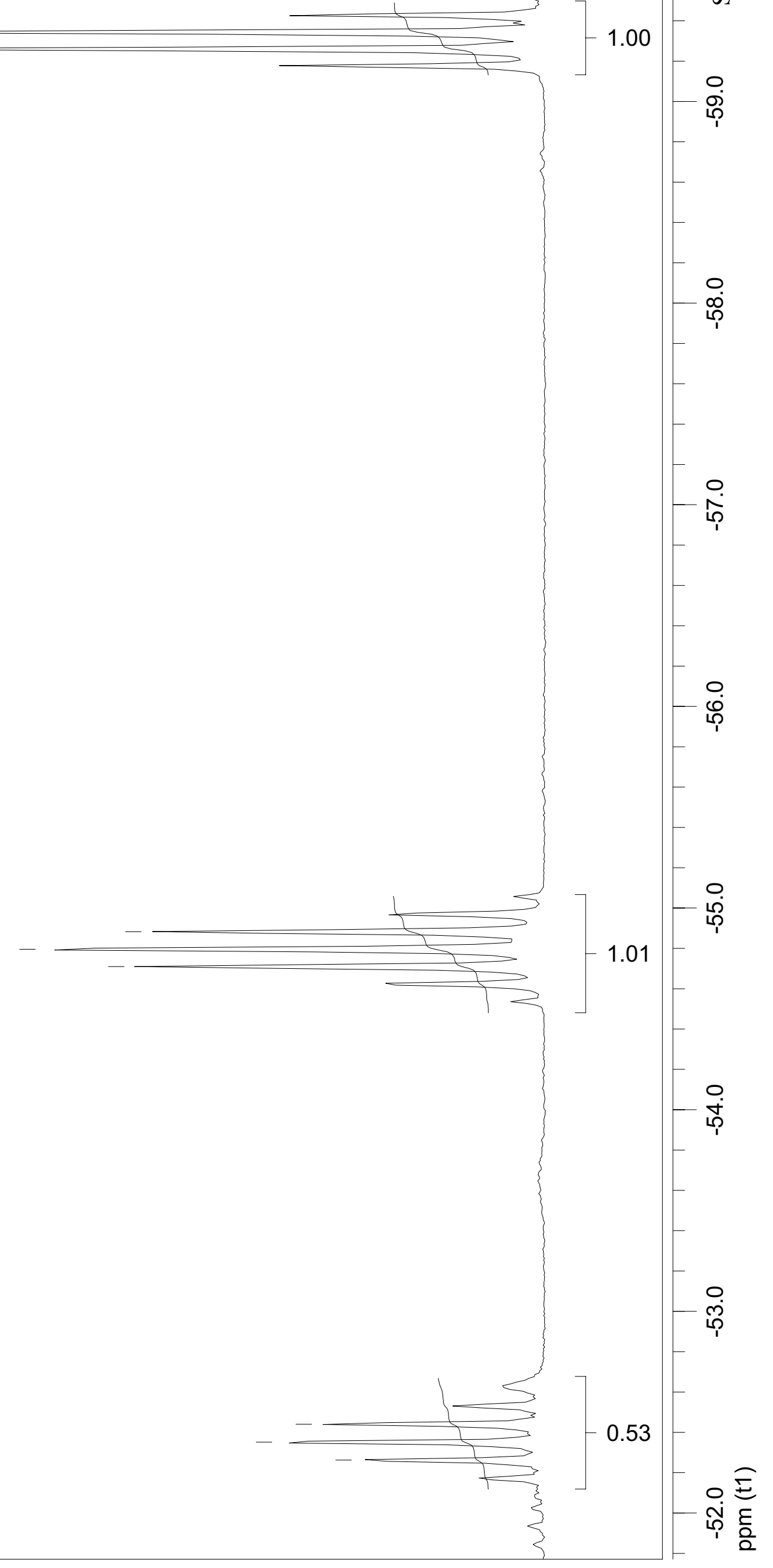
OS'19-

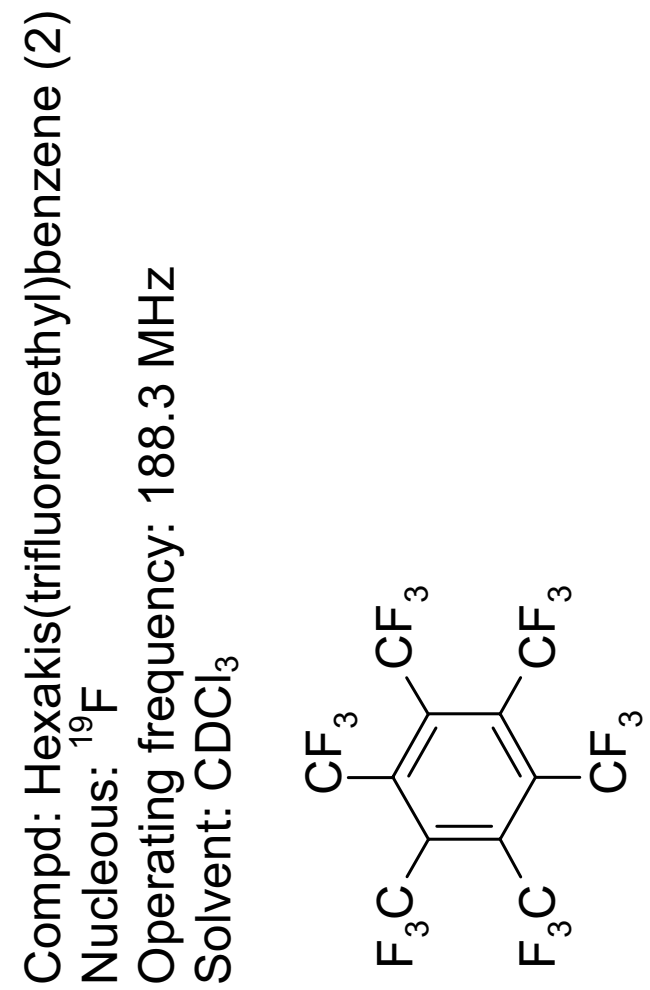

$\neg 0^{\circ} \angle G^{-}$

$16^{\prime} \downarrow 9^{-}$

เG'tG- 


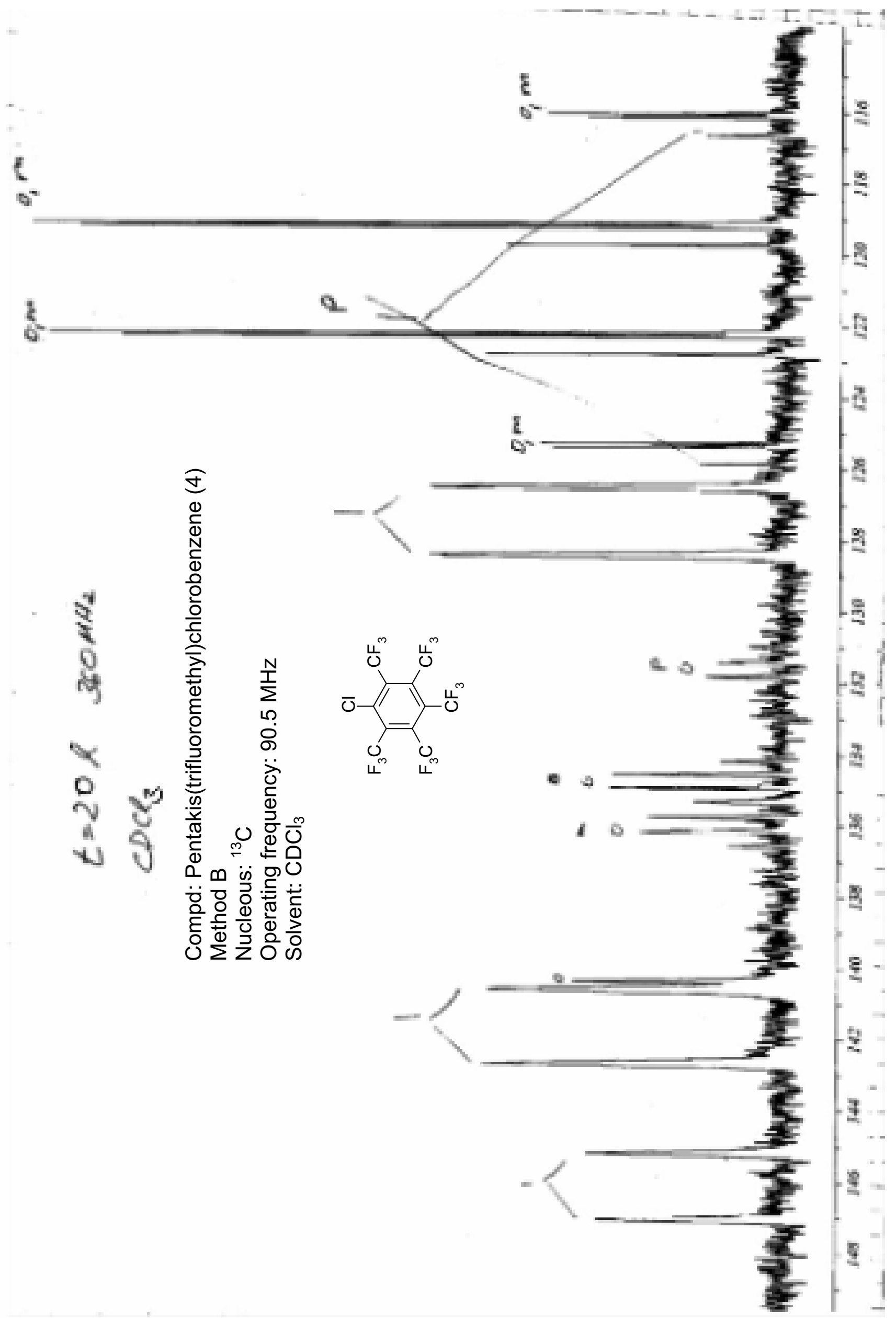


$9 \tau \varepsilon \angle \angle S-$

II $+9^{\circ} \angle S-$

$S 6+S^{\circ} \angle S-$

$88 t^{\circ} \angle S^{-}$

$\Im$

$\stackrel{0}{c}$

밈

$\stackrel{N}{\frac{C}{10}}$

ฮั่

응

은

产

$\bar{x}$

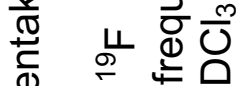

ه $\infty$ is

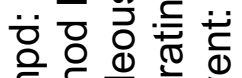

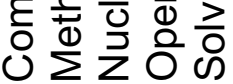

$I S \angle t^{\circ} \mathcal{E} S-$

$9 \mathcal{E} 8 \mathcal{E}^{\circ} \mathcal{E} \mathcal{S}^{-}$

$0 Z 6 Z^{\circ} \mathcal{E S}-$

SOOZ 'ES-

$680 I^{\circ} \mathcal{E S}-$

$\mathcal{E} L I 0^{\circ} \mathcal{E} \mathcal{S}^{-}$

$2+8 I^{\circ} \mathrm{Zs}-$

9260.25

$0100.25-$

$5606^{\circ} \mathrm{IS}$

$6 \angle I 8^{\circ} \mathrm{IS}-$

t92L'IS-
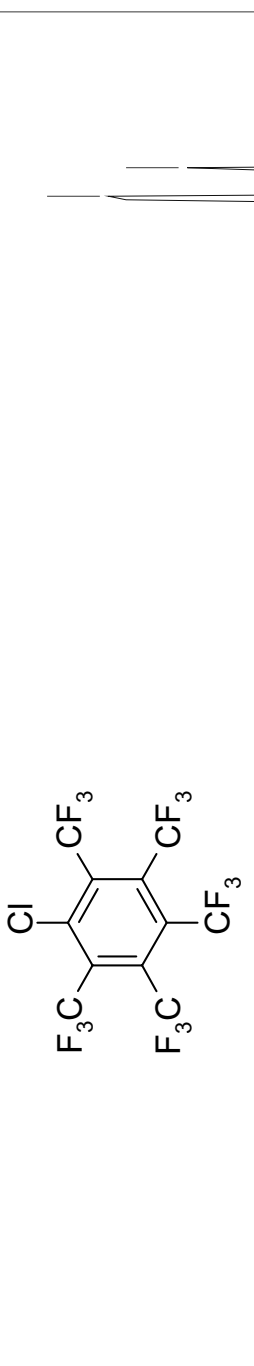
$6 \varepsilon^{\prime} \angle S^{-}$

$6 Z^{\prime} \angle S^{-}$
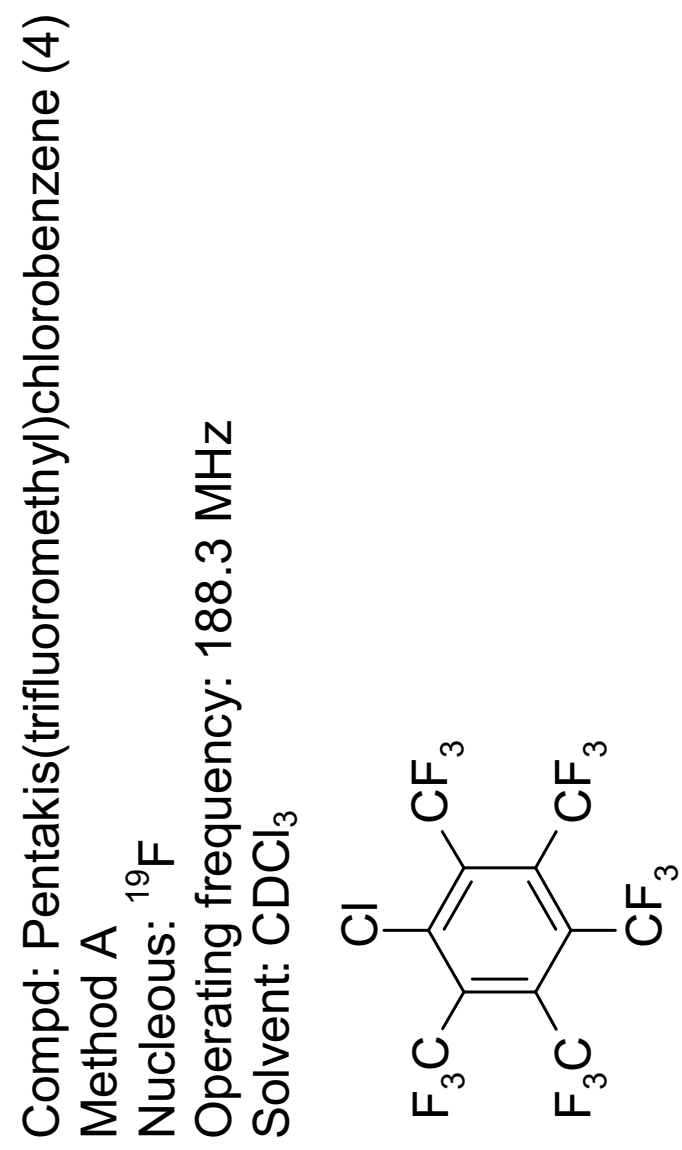

Z0'६G-

¿6'Z9-

†८'Z9-

$\nabla L ' L G$ -

t9' $L$ - $^{-}$

SG'LG-
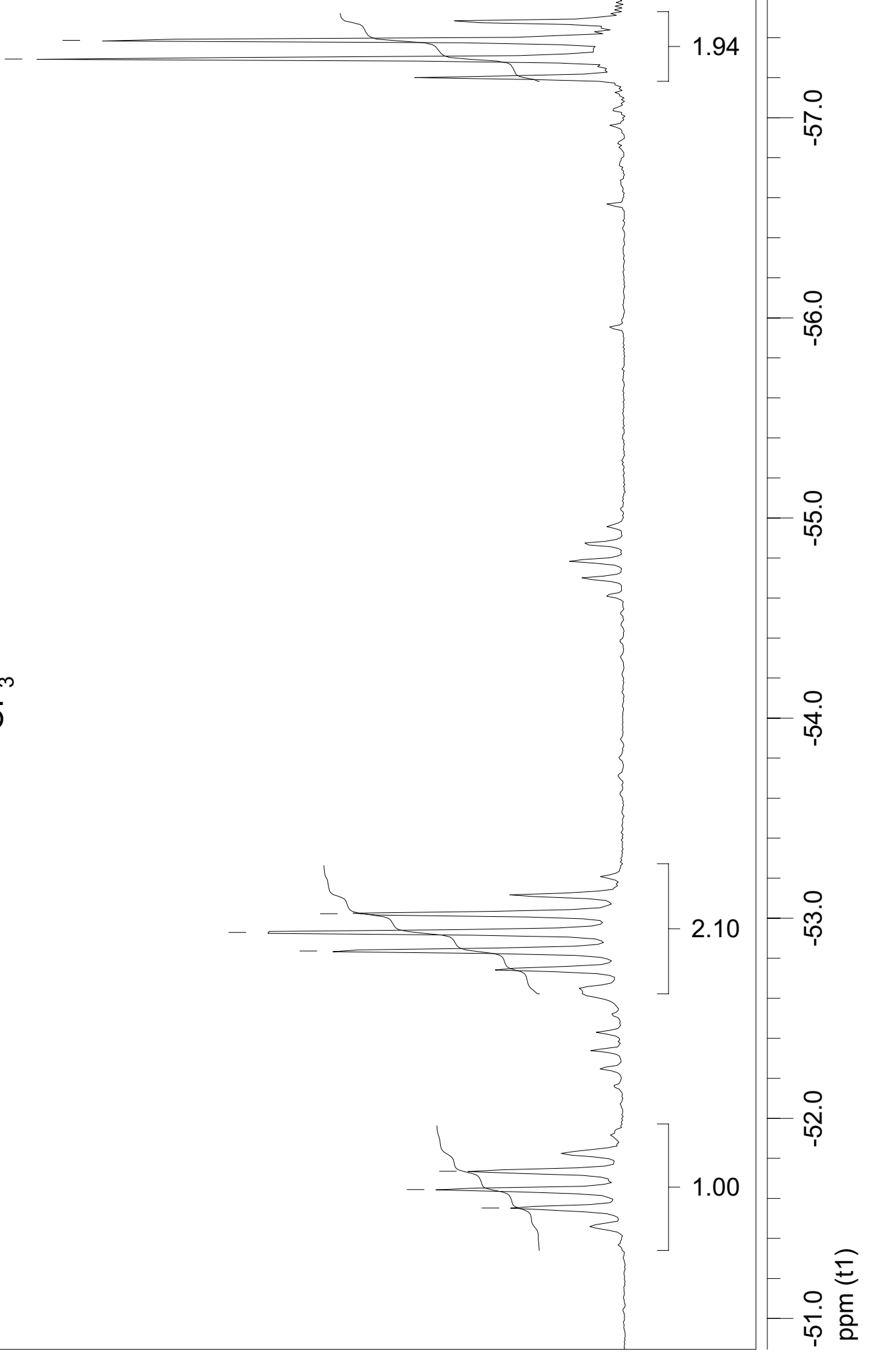


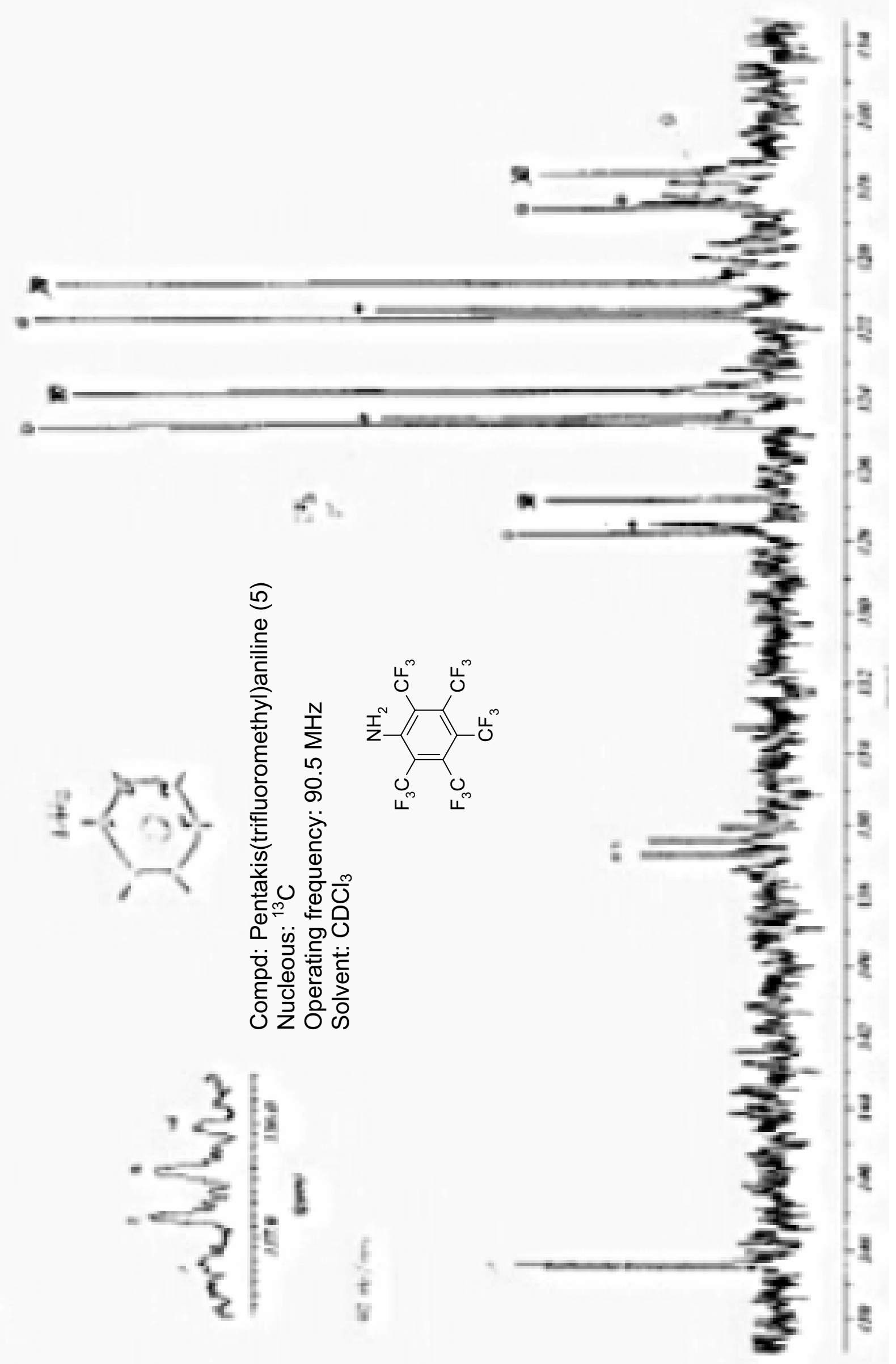




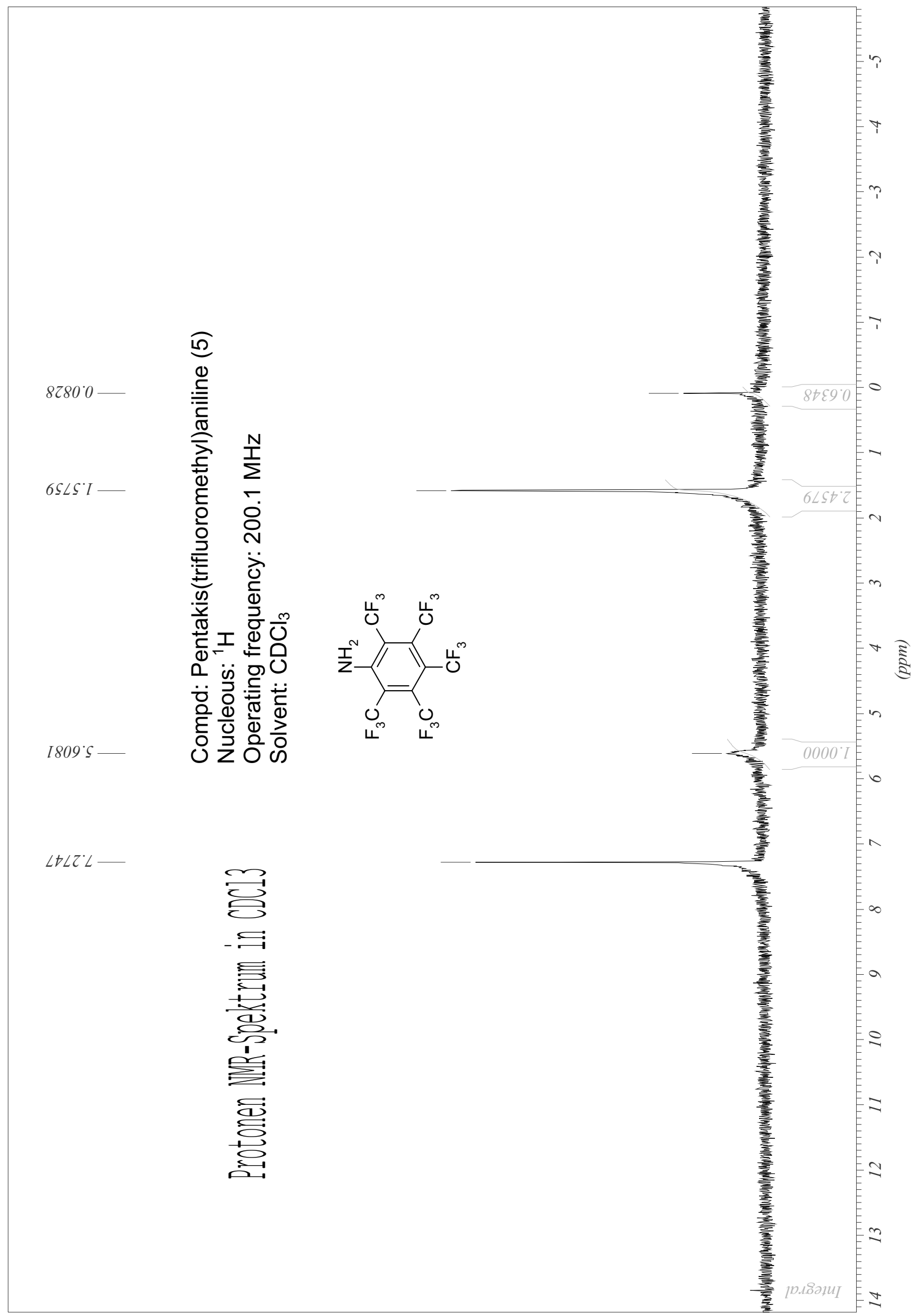




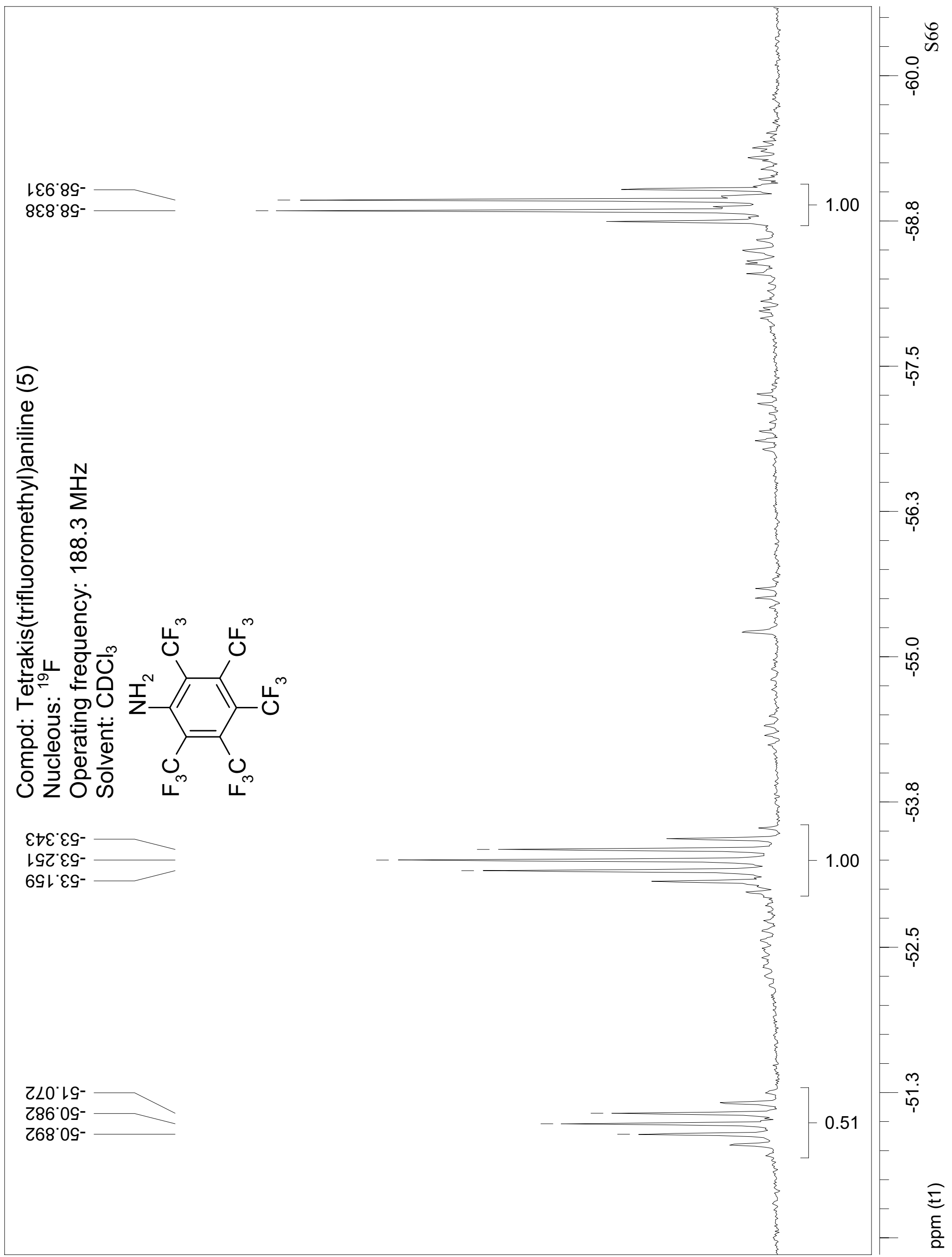


98LடL

$6+8 \angle L$

$\angle t 66^{\circ} 6 \mathrm{LL}$

เ6L.0ZL

LI9OZL

LOL'ZZL

6Е6 ZZレ

$\varepsilon \angle \varepsilon^{\circ} \varepsilon Z \downarrow$

99tตZL

งEl'9ZL

$\left\llcorner 80^{\circ}\llcorner\varepsilon L\right.$

Eカt๋ เEL
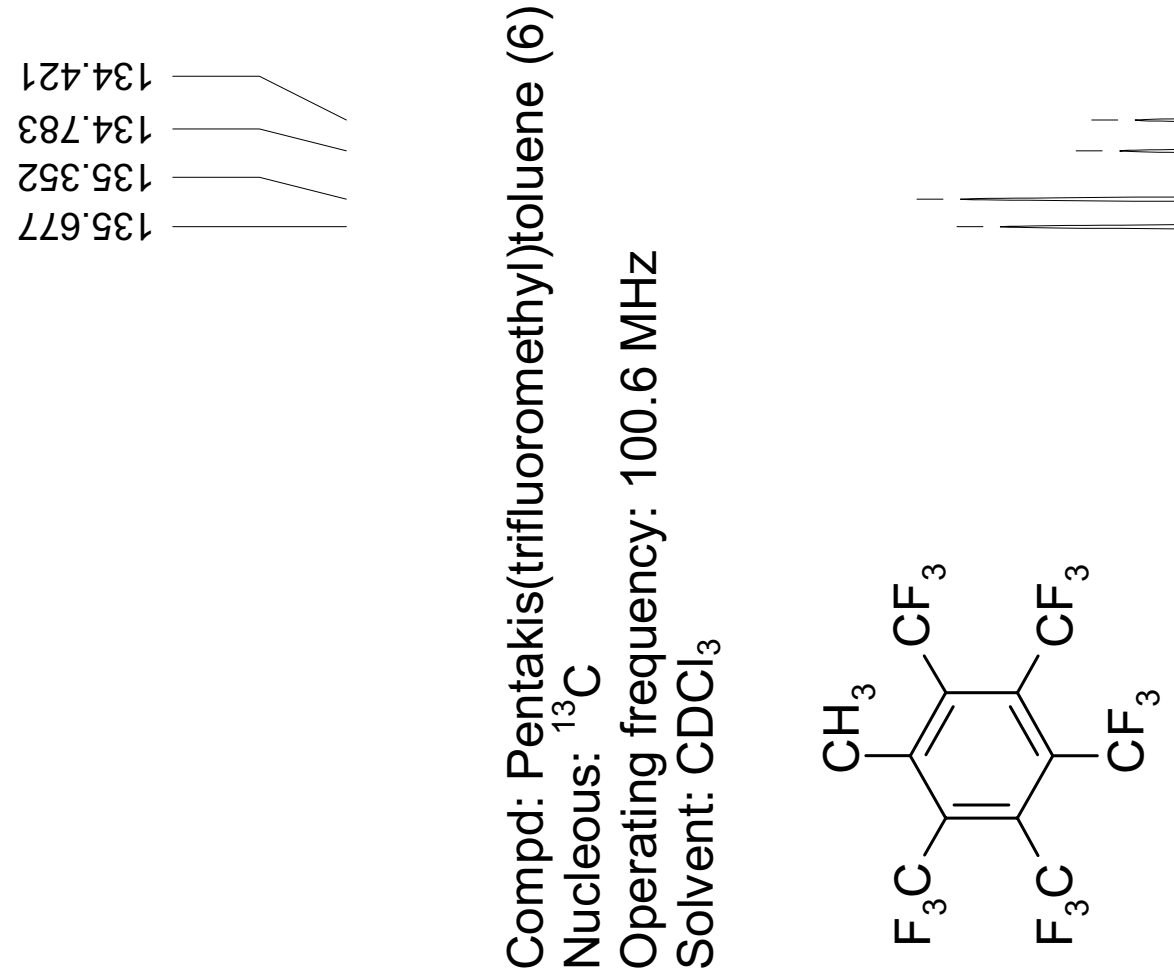

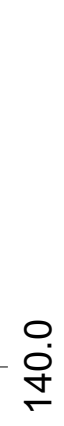

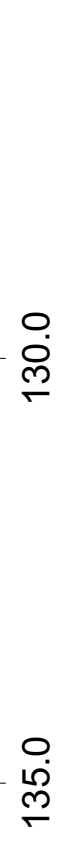

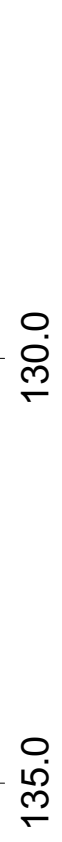

$\stackrel{\circ}{\circ}$

ำ

6

$\frac{10}{ \pm}$ 
$9 \angle 0^{\circ} 0$

SEG.

$\angle E L ' Z$

(6)

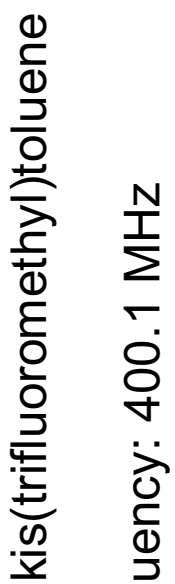

胥I 헝

든

0 is

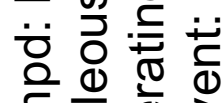

ह $\frac{0}{0} \stackrel{0}{\searrow}$

ல
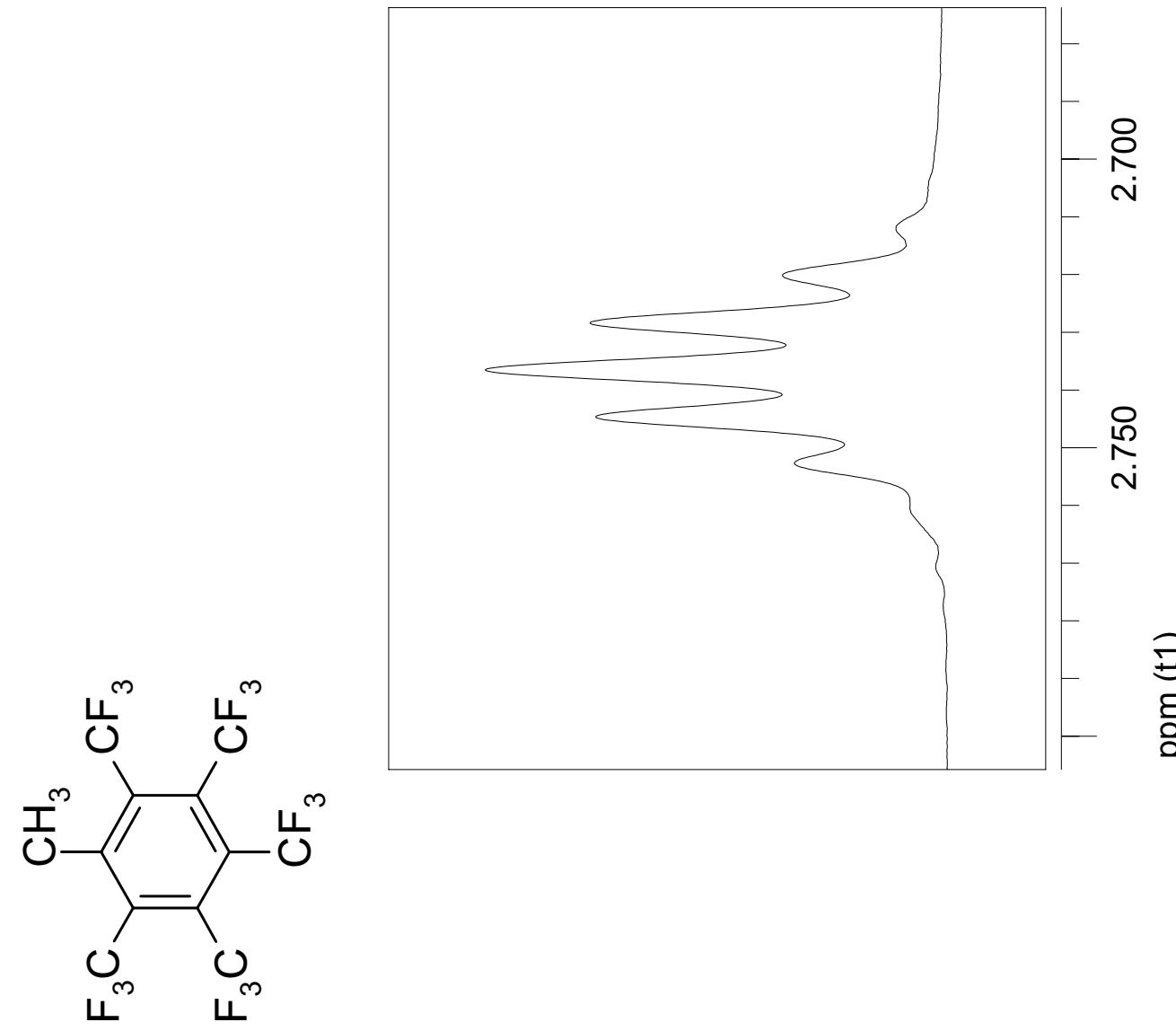

$\stackrel{\circ}{m}$

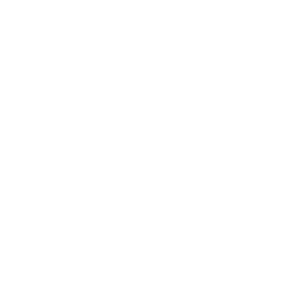


09'99-

เナ'99-

(6)

(1)

(1)

응

言 吉

ह

잉 $\infty$

음 한

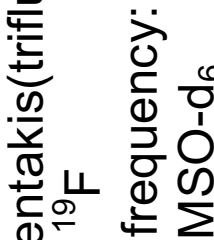

○

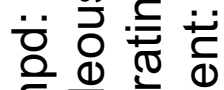

ปั่

$09^{\circ} \varepsilon 9$

IG'ES-

乙† EG-

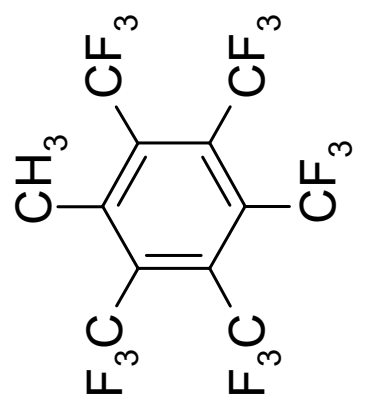

カฤ て৭-

५E'乙G-

9乙 乙९-

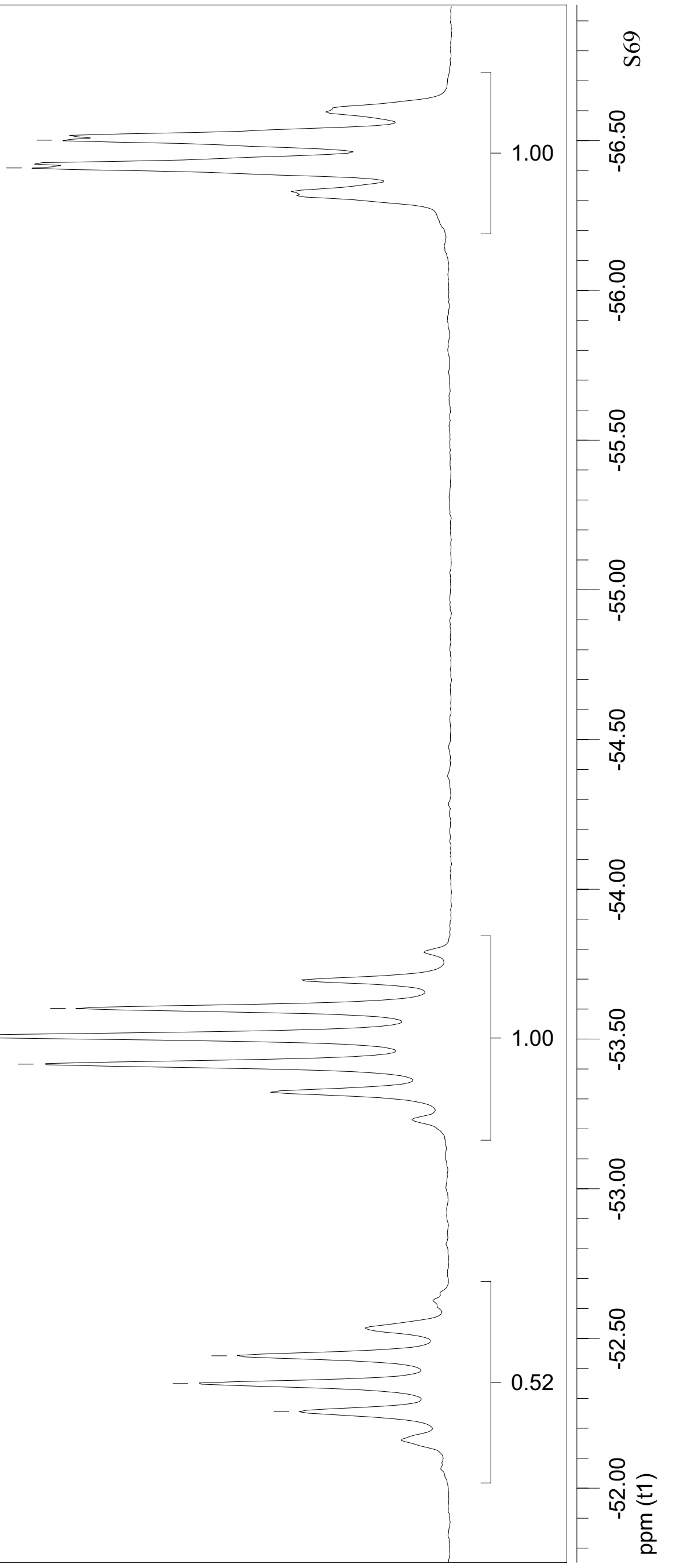




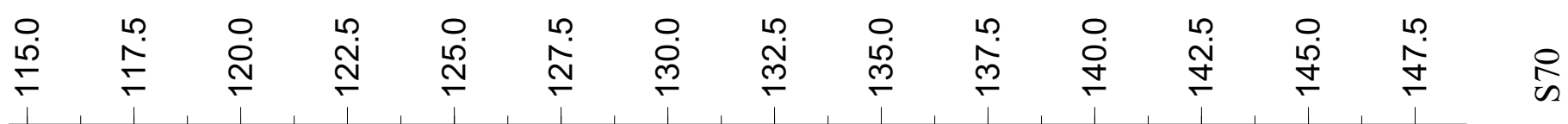

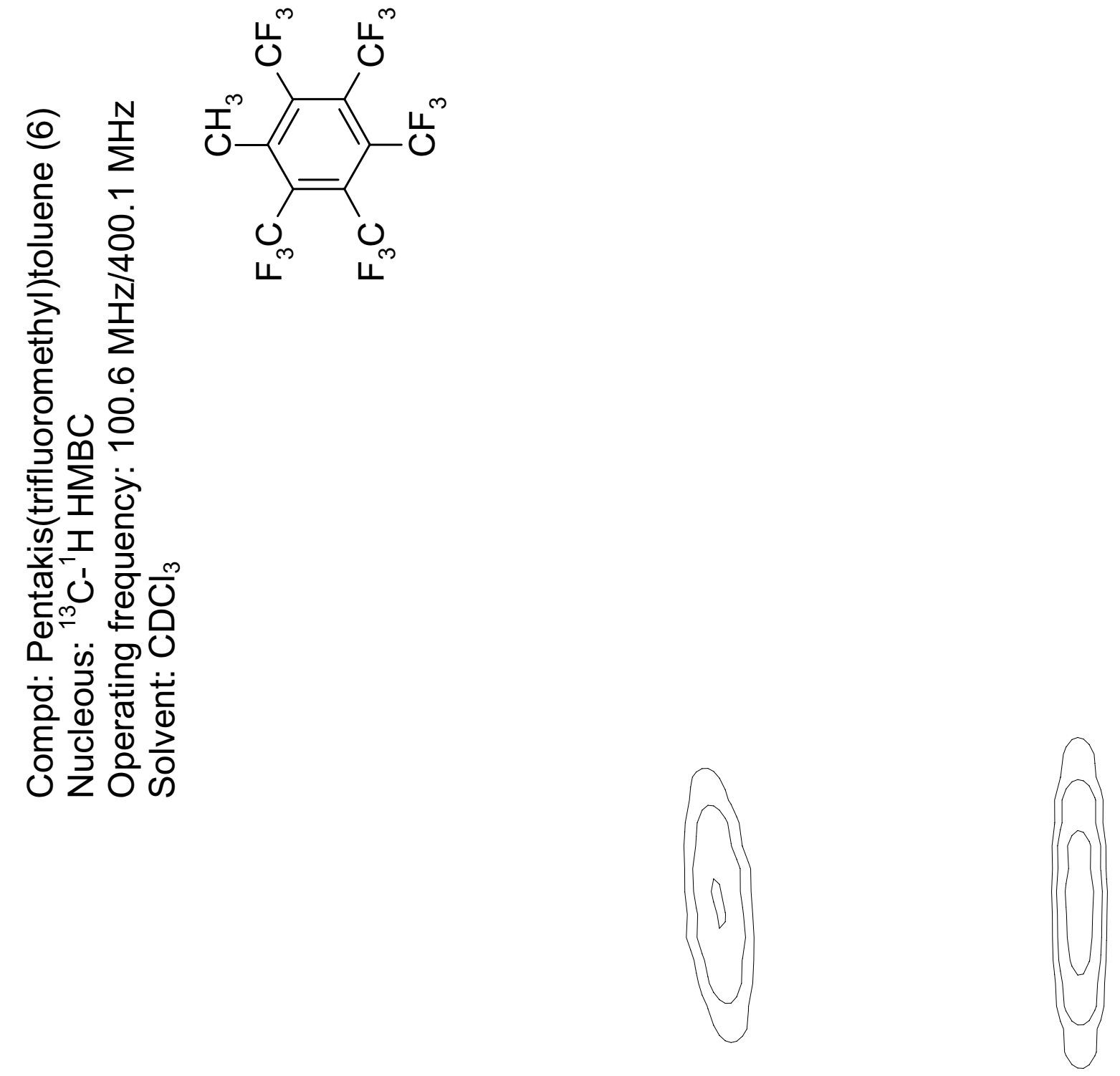


$99^{\circ} L$

$98^{\circ} 62$
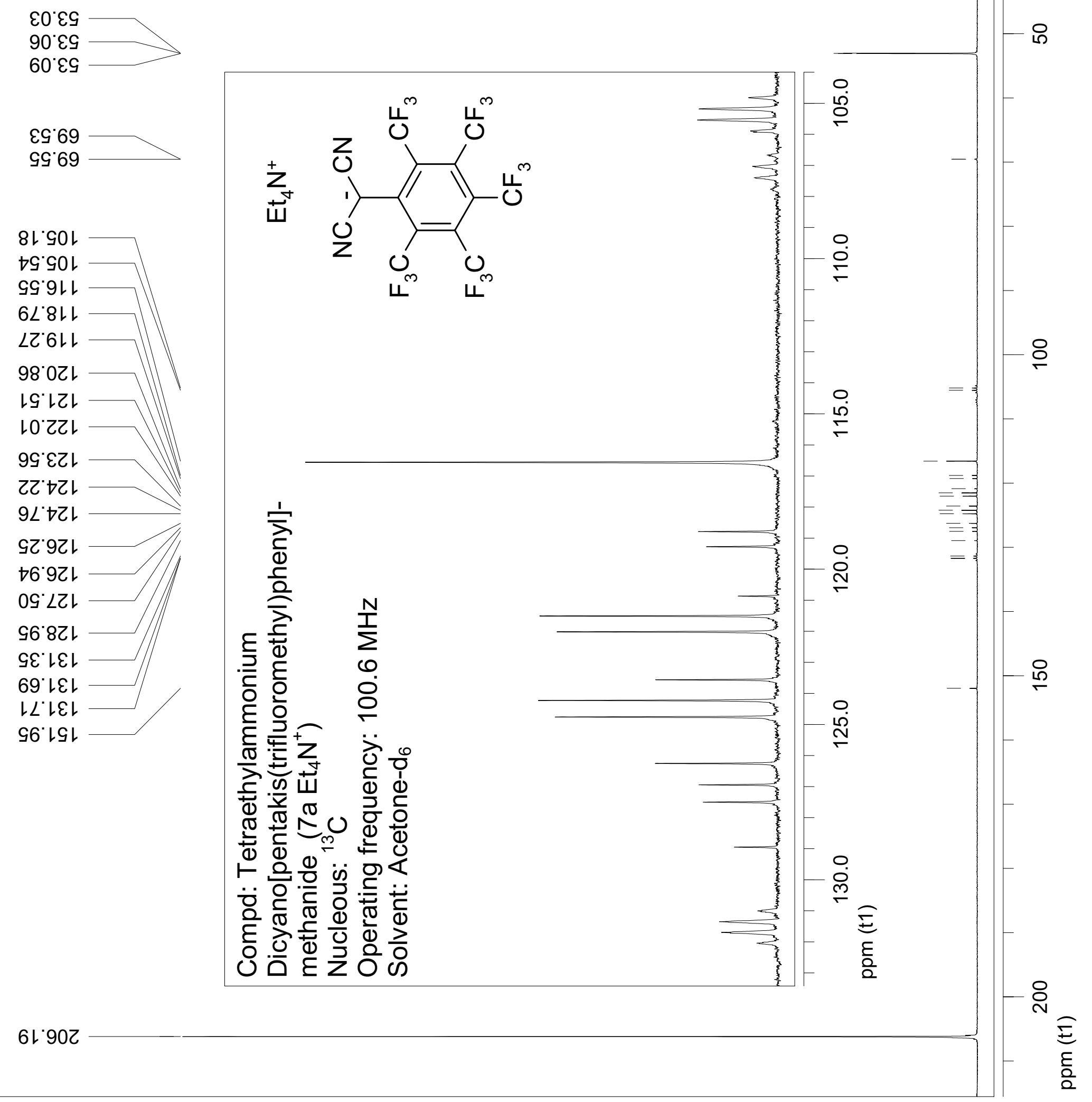
$6 \varepsilon^{\circ}\llcorner$

$0 t$

902

乙८ 2

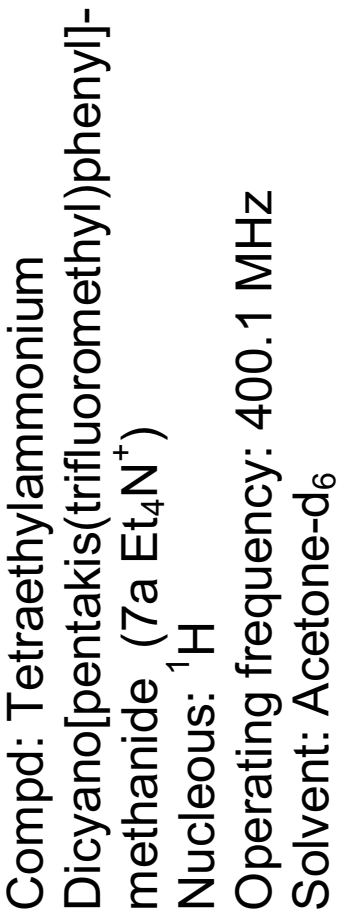

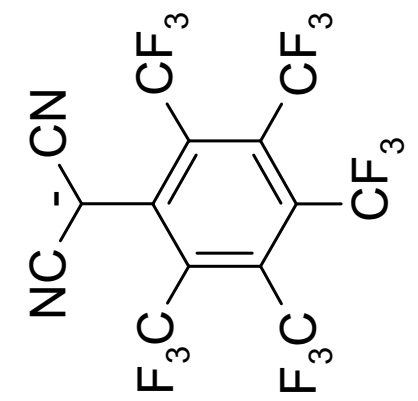

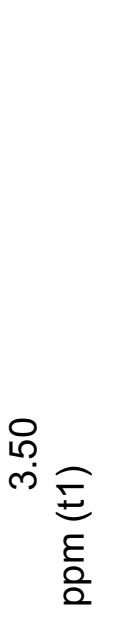


9L6 'LG-

G88' LG-

G6L'L-
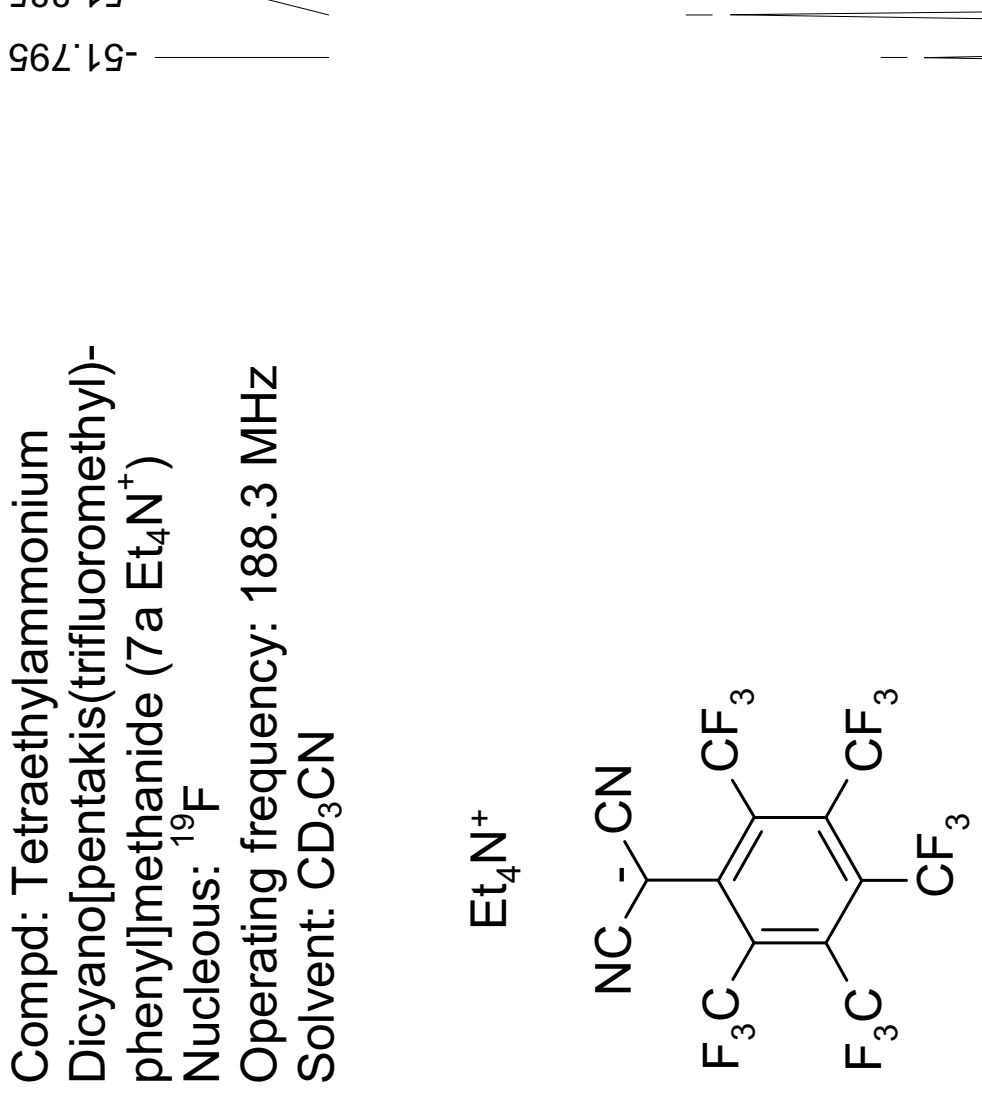

$09 \varepsilon^{\circ} 6 t^{-}$

$692^{\circ} 6 t^{-}$

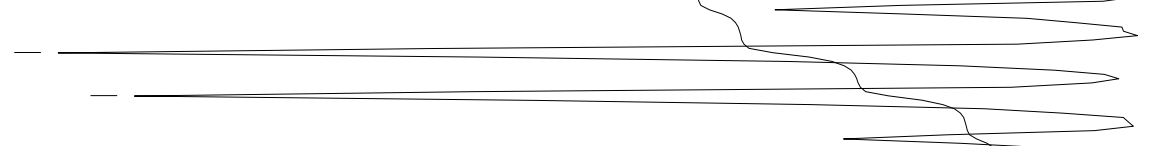




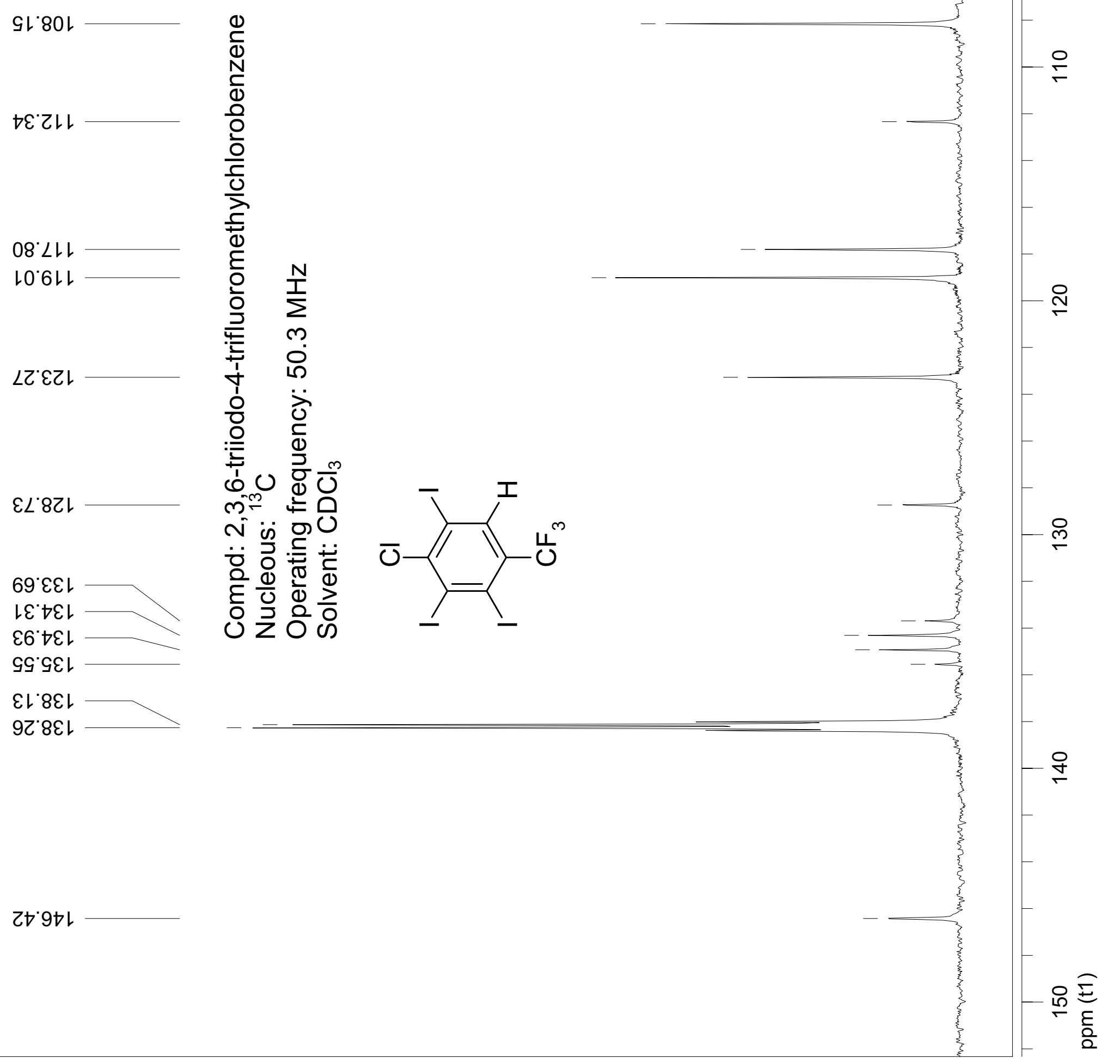


$92^{\circ} L$

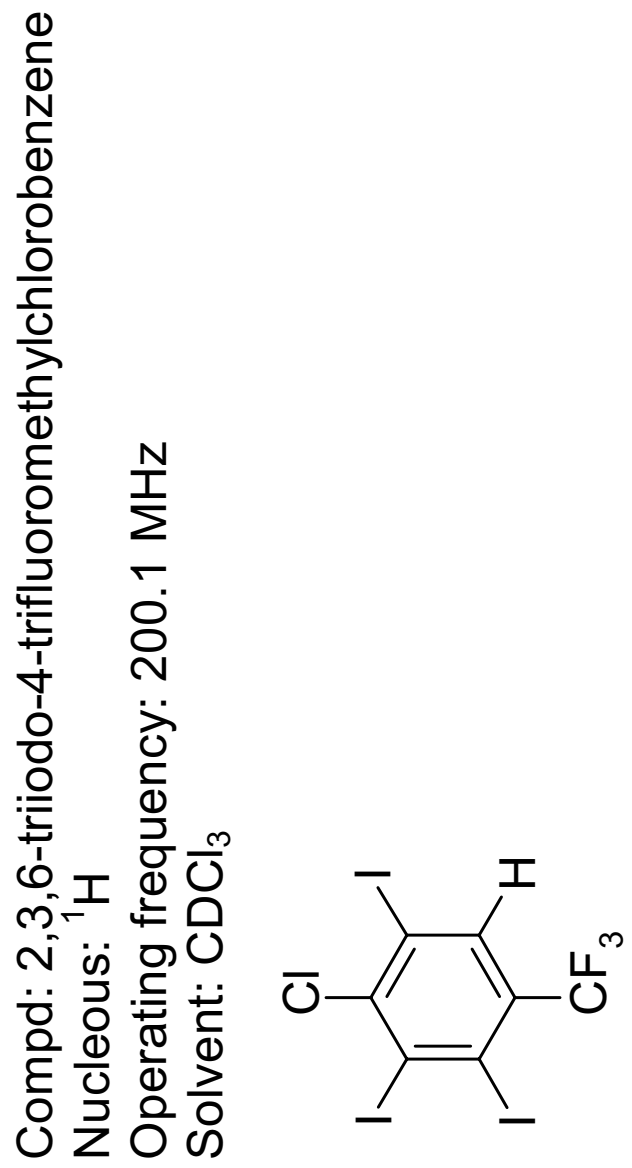

レレ8 
乙๕乙" †9-

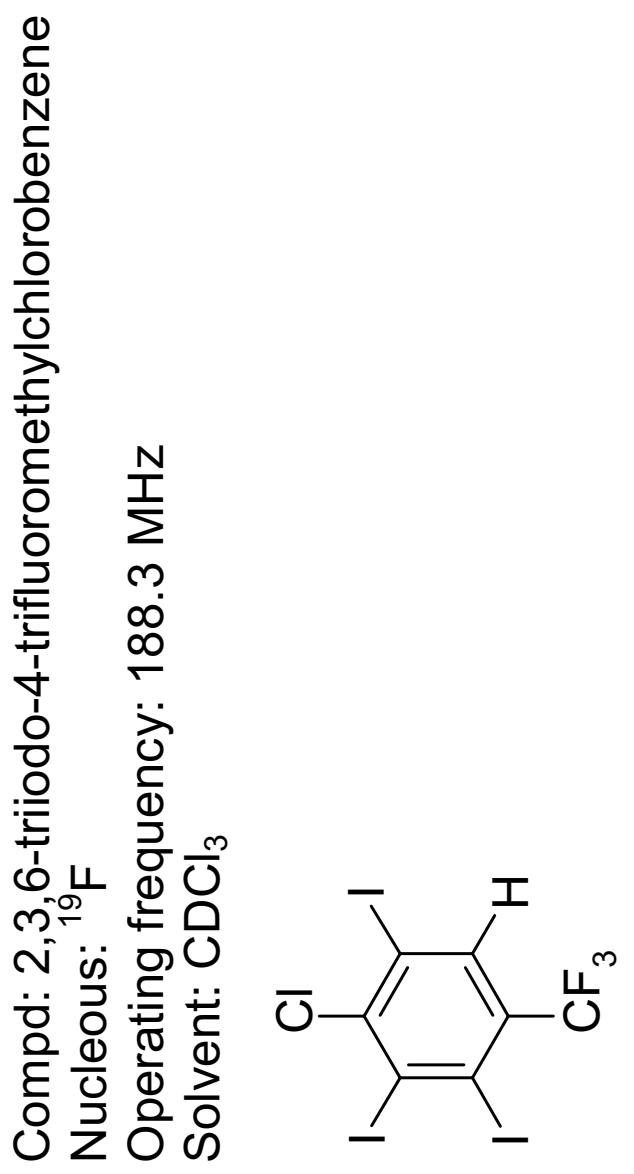

099'\&9- 


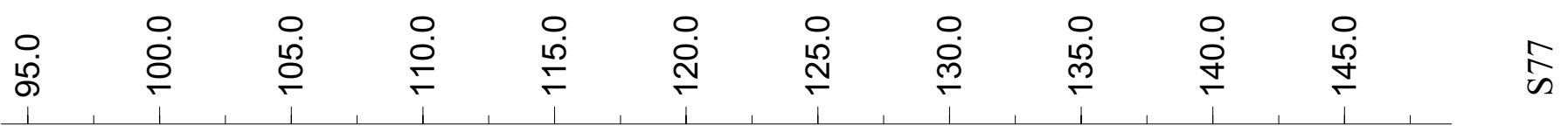

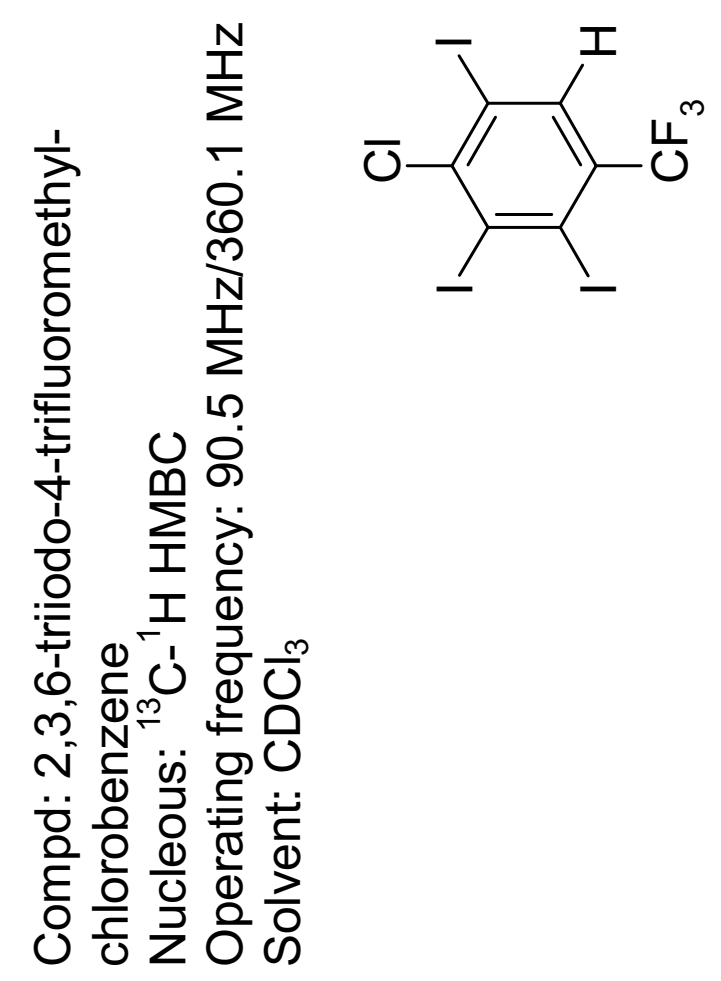

- 유

$\underset{\substack{n \\ \stackrel{\infty}{\infty}}}{\stackrel{n}{\infty}}$

$\stackrel{8}{\stackrel{8}{\circ}}$

$\stackrel{\leftrightarrow}{\circ}$

$N$

용

․

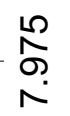

A

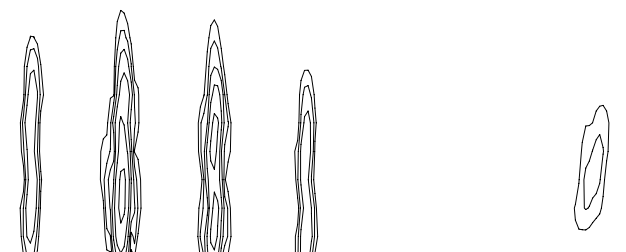

○

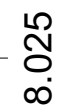

0
0
0
0

$\stackrel{0}{5}$
0
$\infty$

$\frac{8}{\infty}$

$\stackrel{\stackrel{2}{\stackrel{2}{c}}}{\infty}$ 


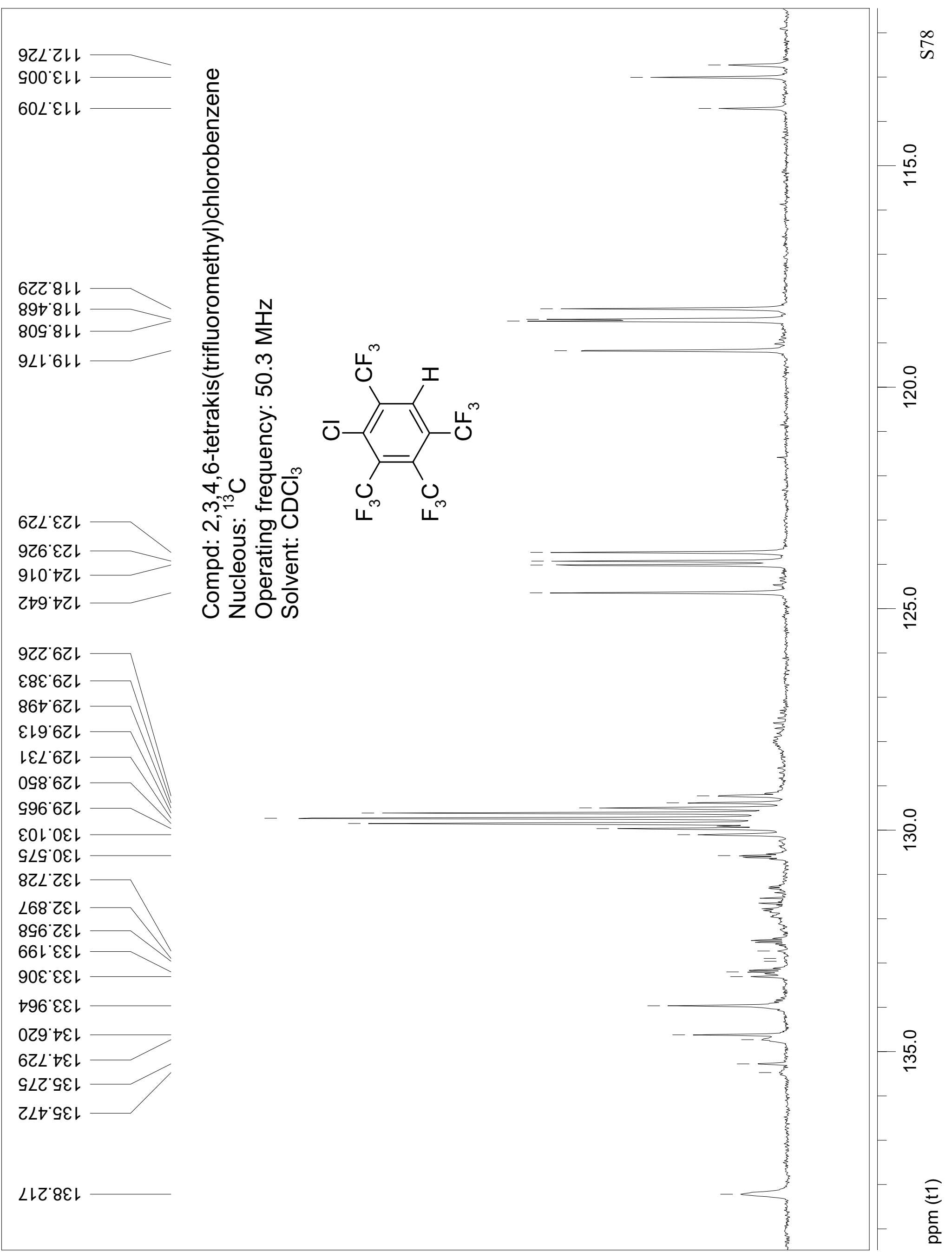




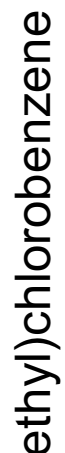

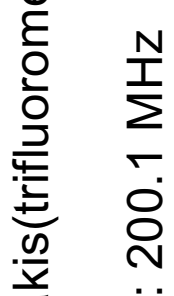

它 它

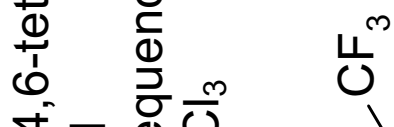

オI

ต๐

iो

을 긍 엉

은 눤

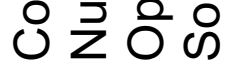

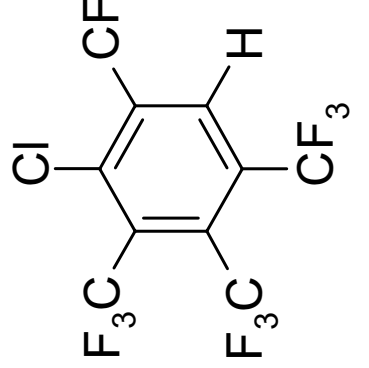

LE'8 


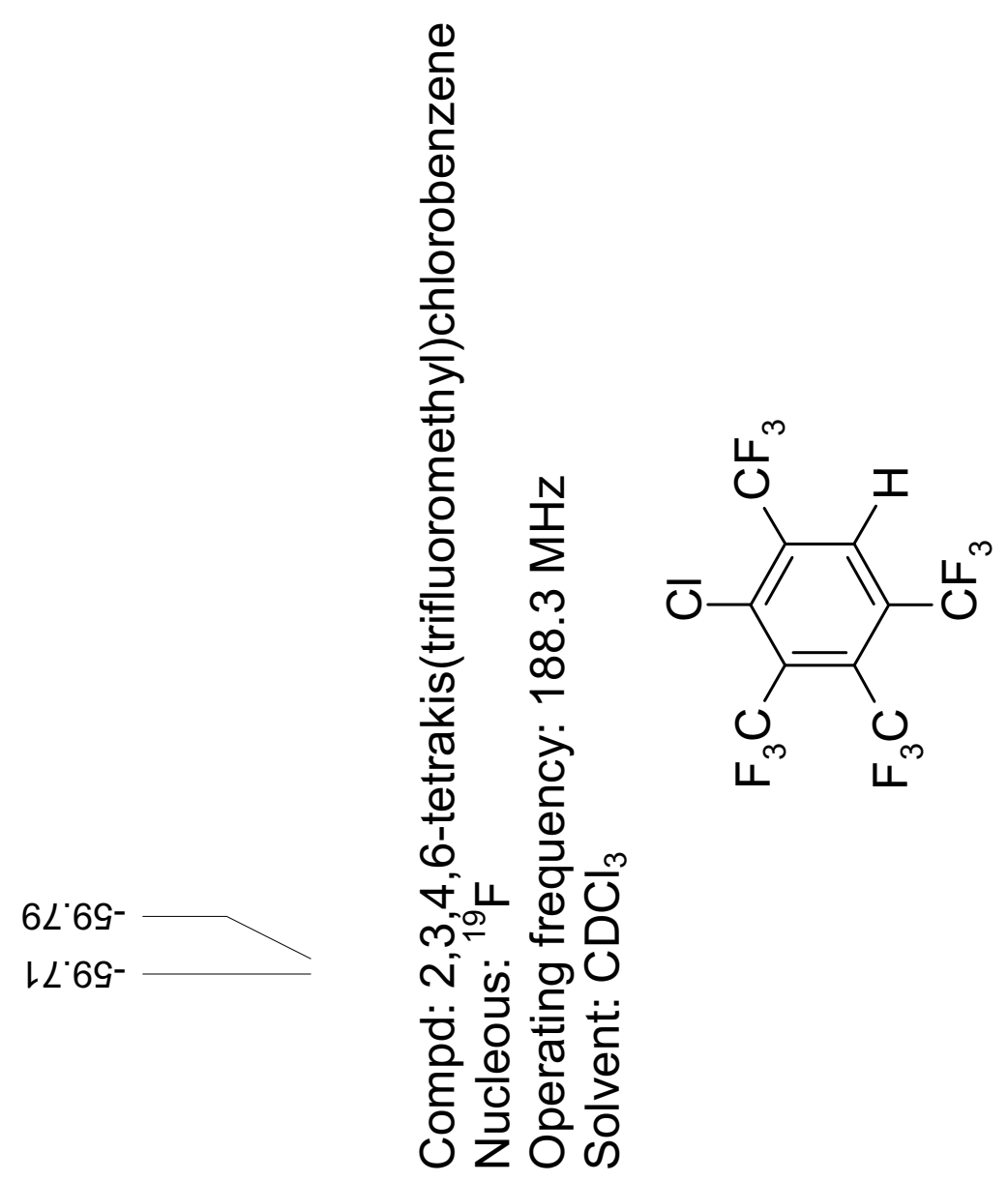

00.89-

$16{ }^{\circ} \angle 9^{-}$

Z8 $\angle 9-$

29.99-

$G^{\prime} G \mathrm{~s}^{-}$

97'Gs-

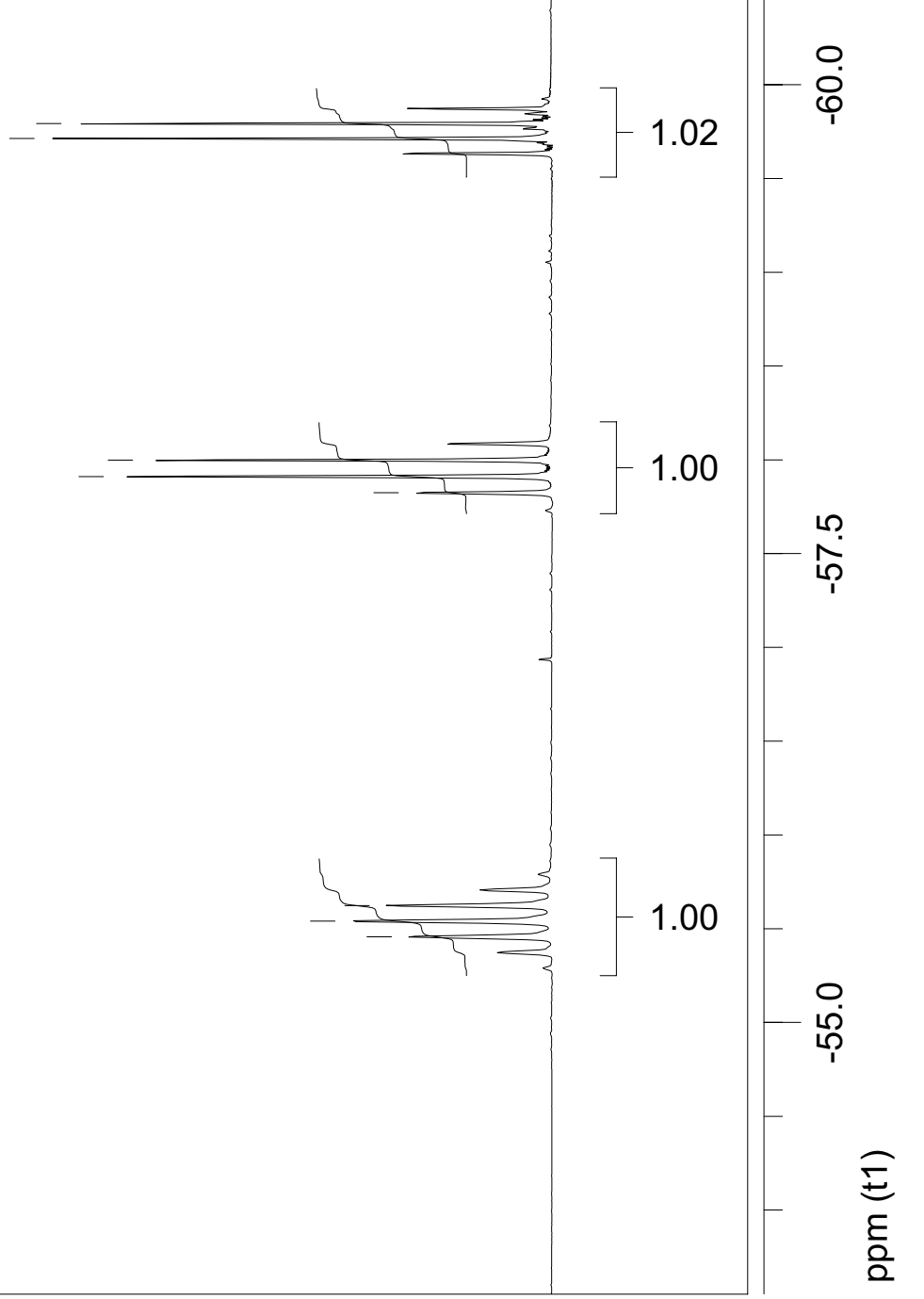




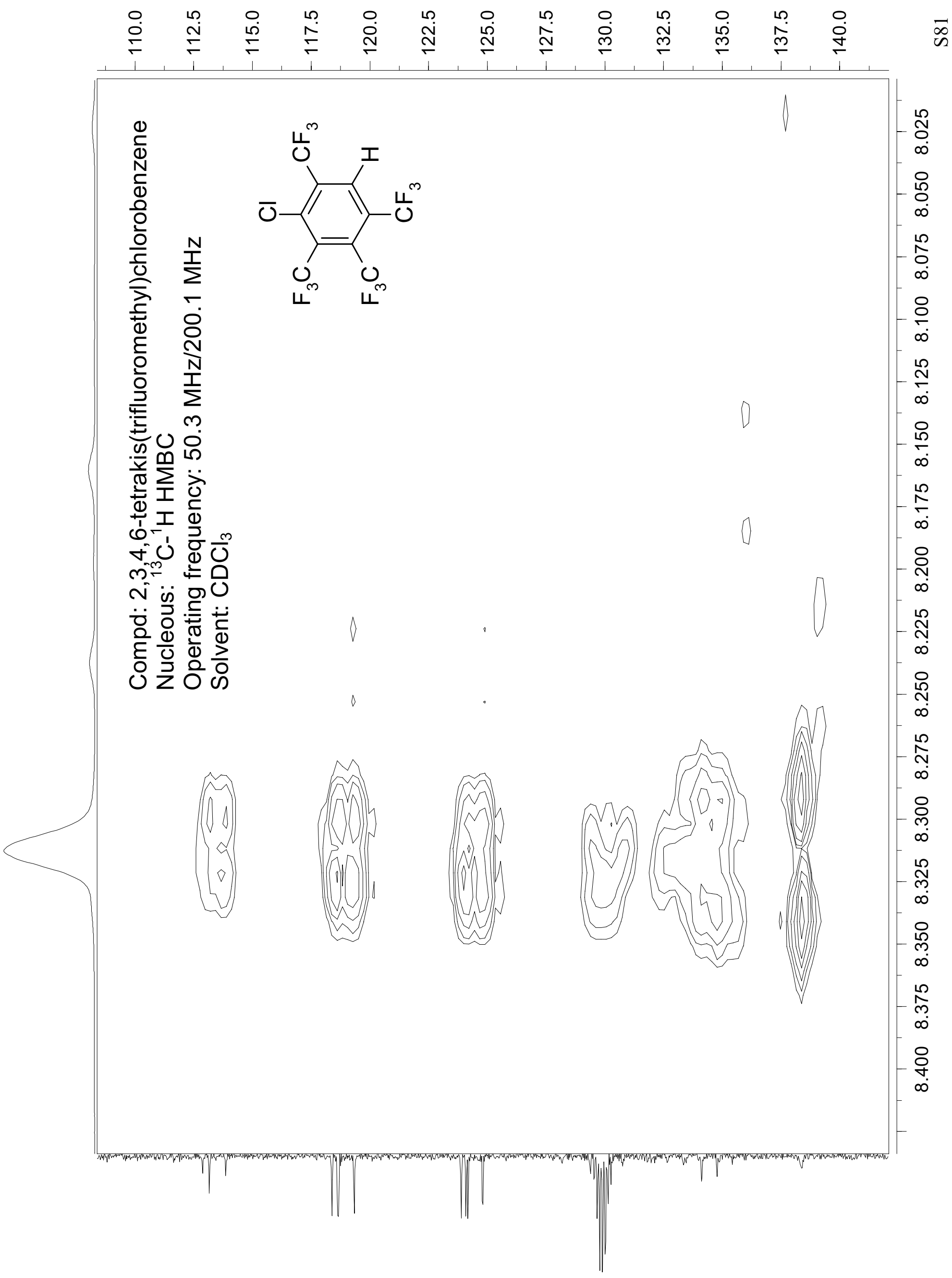


$99^{\circ} \mathrm{L}$

$\angle 8^{\circ} 62$

与 $8: 6 \varepsilon$

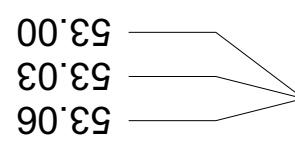

乙乙'6L

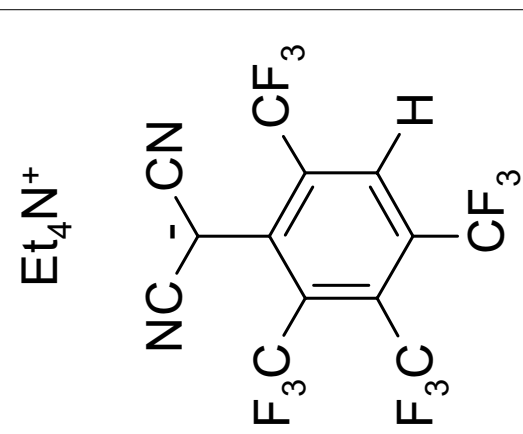

$99 \circ L$

66 나

$706 \mathrm{LL}$

EL $0 Z L$

$\angle Z O Z L$

SO IZL

$8 \varepsilon^{\circ} L Z L$

LL'LZL

06 ZZL

$09^{\circ} \downarrow Z \downarrow$

$0 \varepsilon$ 'GZL

99'9ZL

$\angle 9^{\circ}$ GZL

$\varepsilon Z \angle Z L$

$1062 \mathrm{~L}$

${ }^{\circ} \varepsilon \varepsilon \downarrow$

$6 乙^{\prime} \downarrow \varepsilon L$

88.`ㄴ

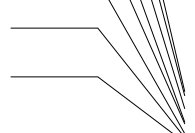

ó

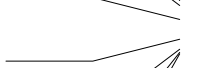

N

능

음 $\frac{\bar{\sigma}}{\frac{1}{5}}$

웜

ป气 $\frac{N}{\Sigma} \stackrel{N}{\Sigma}$

ह

을 등 웅

है 吾 ठें

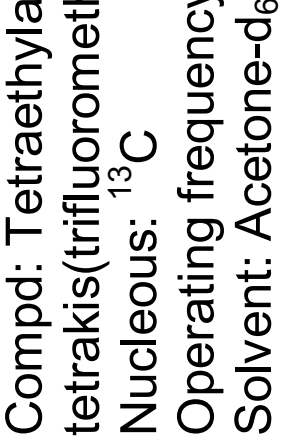


$70^{\circ} \mathrm{Z}$

$90 \mathrm{Z}$

so'

$18^{\circ} 2$

$8 \nabla^{\circ} \varepsilon$

$0 \mathcal{S}^{\circ} \mathcal{\varepsilon}$

ڤ́

ก 1

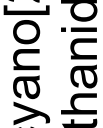

ग्रे

ह $\frac{\mathrm{E}}{\mathrm{N}}$

高 $\frac{\bar{c}}{\bar{c}}$

을응

ह ₹

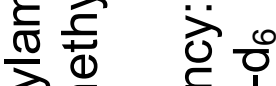

¿

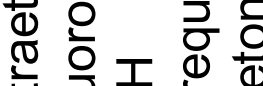

는 근 는

些 i

خृ

을처 인

ह 전

லั๋

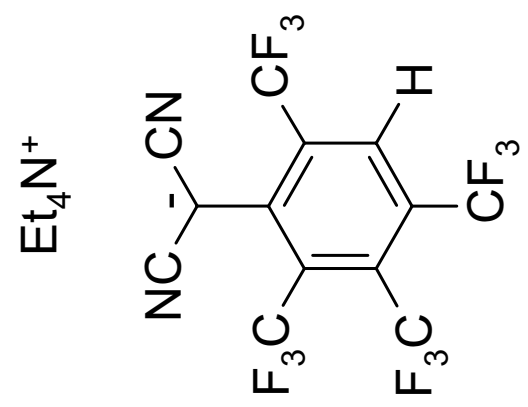

$98^{\circ} \mathrm{L}$

$00^{\circ} 8$ 


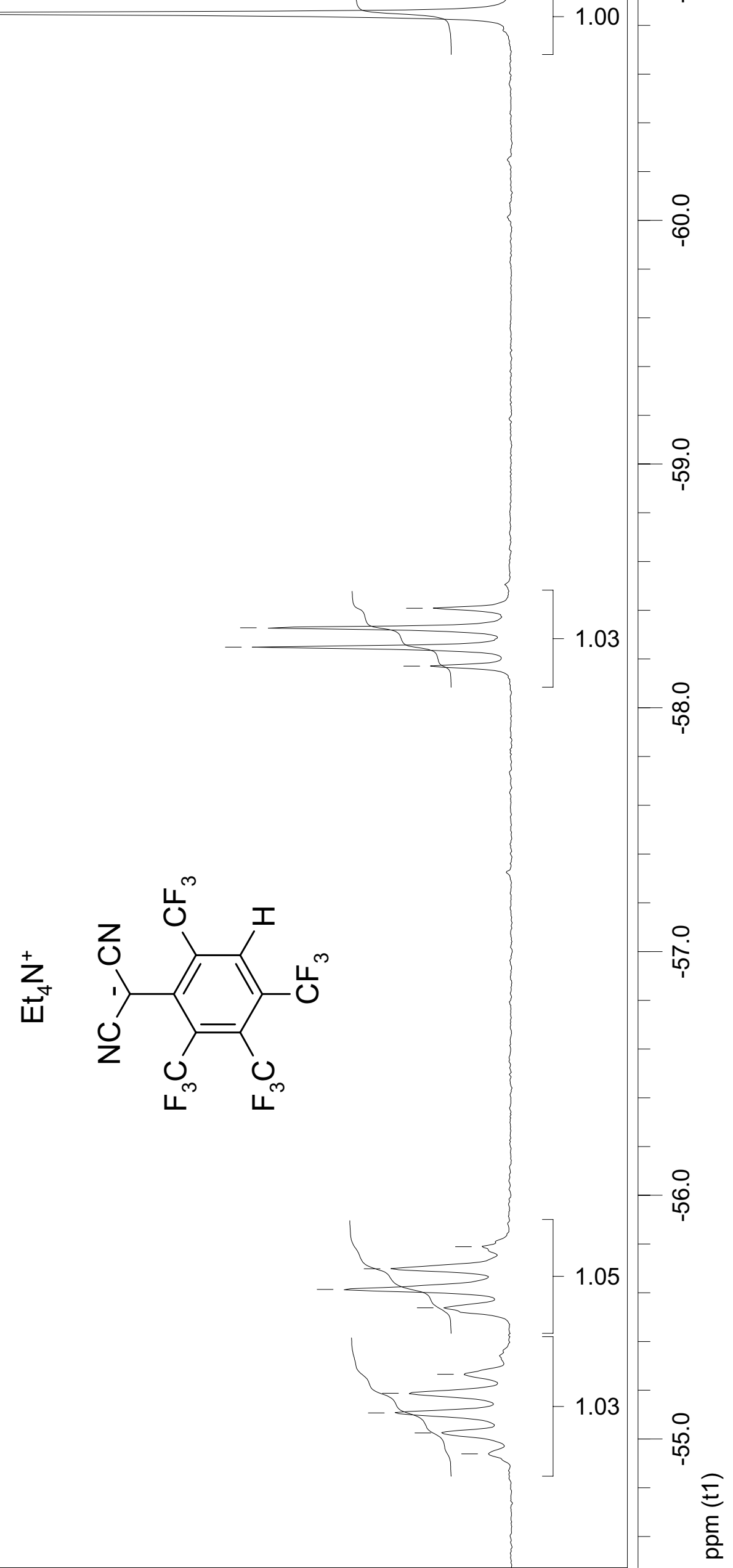




\section{Optimized Geometries}

\section{Obtained from the}

\section{Computations}

\author{
$\mathrm{C}_{6}\left(\mathrm{CF}_{3}\right)_{5} \mathrm{H}$
}

Brutoformula: C11H1F15

0,1

$\mathrm{H}, 0.2376605249,0 ., 2.8892129419$

C, $0.1263062324,0 ., 1.8164589032$

C, $0.0538255271,0 .,-0.9532026037$

C, $0.0457968684,1.2136576179,1.148577992$

C, $0.0457968684,-1.2136576179,1.148577992$

C,-0.0351105071,-1.2265705503,-0.2541821126

C, $-0.0351105071,1.2265705503,-0.2541821126$

C, $0.0858267896,2.4522692631,2.0507713304$

C, $0.0858267896,-2.4522692631,2.0507713304$

C, $-0.3073450315,-2.5526354055,-0.9914932289$

C, $-0.3073450315,2.5526354055,-0.9914932289$

C, $0.4220341071,0 .,-2.4551373289$

F,-1.148101744,2.8882370416,2.3601155041

$F, 0.7785854129,3.4664035533,1.5147462406$

$F, 0.6928523455,2.1491018442,3.2208946331$

F,-0.9461152782,2.3494375932,-2.1547895639

F, $0.8041185611,3.2587415882,-1.2367381448$

$\mathrm{F},-1.1311053852,3.3203435652,-0.2522615817$

$\mathrm{F},-0.6212286887,0 .,-3.2902695501$

F, 1.1870097013,-1.0718119779,-2.7391664532

F, 1.1870097013,1.0718119779,-2.7391664532

F,-0.9461152782,-2.3494375932,-2.1547895639

F,-1.1311053852,-3.3203435652,-0.2522615817

$F, 0.8041185611,-3.2587415882,-1.2367381448$

$\mathrm{F},-1.148101744,-2.8882370416,2.3601155041$

$F, 0.6928523455,-2.1491018442,3.2208946331$

F, $0.7785854129,-3.4664035533,1.5147462406$

$H F=-1917.9805365 \quad \mathrm{NImag}=0$

$\mathrm{C}_{6}\left(\mathrm{CF}_{3}\right)_{5}{ }^{-}$

Brutoformula: C11F15(1-)

$-1,1$

$F, 1.5294611113,1.0778632583,-2.1058349983$

C, $-0.1830303188,-0.6864743698,-1.0010209888$

C, $0.0177665251,-0.743875471,1.7531078986$

C,-0.3330261181,-1.8781692562,-0.3497078337

C, $0.0892664622,0.4763228867,-0.3330097132$

C, $-0.1342273957,0.4989778609,1.0776455184$

C, $0.0933823855,-1.9497244919,1.0124196872$

C, $-1.0161025287,-3.0053524625,-1.0847419827$

C, $0.7549972965,1.5856216955,-1.1177803381$

C, $-0.60515081,1.7711702338,1.7527001955$

C, $0.7188876435,-3.230833997,1.5336916068$
C, $0.1753466916,-0.774991403,3.2675762173$

F,-0.2091137379,-3.6706448331,-1.9520930785

F,-2.0556488814,-2.5468960879,-1.8250682663

F,-1.5331690806,-3.94401278,-0.2484742121

F, 1.7970319166,-3.0110584604,2.3343897133

F, 1.1866068662,-3.9744514951,0.4999200591

F,-0.1151665564,-4.0443522328,2.225852726

F, 1.4337939612,-0.4383213033,3.6547066422

$F,-0.0809999616,-1.9875799629,3.8041846083$

F,- $0.6586088441,0.0705170954,3.9097798122$

$\mathrm{F}, 0.2202836274,2.2231444825,2.7309200126$

F,-1.844046202,1.6486178458,2.3089833281

F,- $0.724844077,2.7863521627,0.8685611407$

F, 1.5819722492,2.3478628383,-0.352209914

$\mathrm{F},-0.1056607818,2.4419577978,-1.7295223314$

$H F=-1917.4230237 \quad \mathrm{NImag}=0$

$\mathrm{C}_{6} \mathrm{H}_{6}$

Brutoformula: $\mathrm{C} 6 \mathrm{H} 6$

0,1

$\mathrm{H}, 0 .,-0.0000031864,-2.4795712089$

C, $0 .,-0.0000068069,-1.3950268849$

C, $0 ., 0.0000068069,1.3950268849$

C, $0 .,-1.2081707586,-0.6975374089$

C, $0 ., 1.2081318616,-0.6975080032$

C, $0 ., 1.2081707586,0.6975374089$

C, $0 .,-1.2081318616,0.6975080032$

$\mathrm{H}, 0 .,-2.1474092216,-1.2398067521$

$\mathrm{H}, 0 ., 2.1473727829,-1.239783655$

$\mathrm{H}, 0 ., 2.1474092216,1.2398067521$

$\mathrm{H}, 0 .,-2.1473727829,1.239783655$

$\mathrm{H}, 0 ., 0.0000031864,2.4795712089$

$H F=-232.3112452 \quad \mathrm{NImag}=0$

$\mathrm{C}_{6} \mathrm{H}_{5}{ }^{-}$

Brutoformula: $\mathrm{C} 6 \mathrm{H} 5(1-)$

$-1,1$

$\mathrm{H}, 0 ., 0.0000000017,2.2786659852$

C, $0 .,-0.0000000022,-1.7205315044$

C, $0 .,-0.0000000021,1.1907989697$

C,-1.1771609187,-0.0000000032,-0.9296286898

C, $1.1771609187,0.0000000002,-0.9296286898$

C, $1.1994638343,-0.0000000038,0.4729388809$

C, $-1.1994638342,-0.0000000014,0.4729388809$

$\mathrm{H},-2.1521524573,0.0000000033,-1.4293386033$

$\mathrm{H}, 2.1521524573,0.0000000034,-1.4293386034$

$\mathrm{H}, 2.1498936694,0.0000000017,1.011561687$

$\mathrm{H},-2.1498936695,0.0000000024,1.011561687$

$H F=-231.6598288 \quad \mathrm{NImag}=0$

$\mathrm{CF}_{3}-\mathrm{C}_{6} \mathrm{H}_{5}$

Brutoformula: $\mathrm{C} 7 \mathrm{H} 5 \mathrm{~F} 3$

0,1 
$\mathrm{H}, 2.1466941616,0.0307688779,-0.5315570426$

C, $1.2109856197,0.0168752682,0.013044706$

C,-1.2073703277,-0.0013998157,1.4052580399

C, $0.0000000002,0.0284647287,-0.6805398023$

C, $1.2073703277,-0.0013998157,1.4052580399$

C,0.,-0.011406342,2.1019825033

C,-1.2109856193,0.0168752681,0.0130447061

C, $-0.0000000001,-0.0044788365,-2.1844743116$

$\mathrm{H}, 2.1473584614,-0.0055383276,1.9445053074$

$\mathrm{H}, 0.0000000002,-0.0239336,3.185889045$

$\mathrm{H},-2.1466941612,0.0307688779,-0.5315570426$

$\mathrm{H},-2.1473584619,-0.0055383276,1.9445053067$

F,- $0.0000000002,-1.2756646612,-2.6626816107$

$F, 1.0880909374,0.6028033523,-2.711338924$

$F,-1.0880909378,0.6028033522,-2.7113389236$

$\mathrm{HF}=-569.4608503 \mathrm{NImag}=0$

\section{2- $\mathrm{CF}_{3}-\mathrm{C}_{6} \mathrm{H}_{4}$}

Brutoformula: C7H4F3(1-)

$-1,1$

$F, 1.1050055476,-0.1004112475,-2.7326590952$ C,-1.4405606474,0.1472917676,-0.0672989538 C, $1.0660330467,-0.1166783022,1.3736120014$ C, $-0.1773345199,0.0218560065,-0.6887155375$ C, $-1.3441082285,0.129173141,1.3454116975$ C, $-0.1474912772,0.0028719198,2.0581895671$ C, $1.0514332773,-0.1070205357,-0.0193870342$ C, $-0.1604802201,0.0291714641,-2.1942159334$ $\mathrm{H},-2.2594880372,0.219367765,1.9380545923$ $\mathrm{H},-0.1515976241,-0.0032301407,3.1493315629$ $\mathrm{H}, 1.9845369526,-0.1990974402,-0.5684151118$ $\mathrm{H}, 2.0040978266,-0.2158071953,1.9127421645$ F,- $0.6515614416,1.1750898192,-2.7467256707$ $\mathrm{F},-0.8784846548,-0.9825770225,-2.7599242491$

$H F=-568.8336964 \quad N I m a g=0$

\section{$3-\mathrm{CF}_{3}-\mathrm{C}_{6} \mathrm{H}_{4}$}

Brutoformula: C7H4F3(1-)

$-1,1$

$\mathrm{H},-2.2688396119,0.0488260251,-0.4117703423$

$\mathrm{C},-1.3531386036,0.0320537541,0.181970967$

C, $1.0498203237,-0.0338212543,1.5398743647$

C, $-0.1421909472,-0.0271237893,-0.5335114055$

C, $-1.4481680297,0.0623464078,1.594352393$

C, $-0.1763039366,0.0275325703,2.217662723$

C, $1.0802034192,-0.0582521644,0.1471761407$

C,- $0.1524818045,0.0032657599,-2.023541485$

$F, 1.0122188758,-0.4356089093,-2.5947315719$ $\mathrm{H},-0.1230905169,0.0479704867,3.3107165945$ $\mathrm{H}, 2.0172042274,-0.1079928382,-0.3954070092$ $\mathrm{H}, 1.9889697983,-0.0643932631,2.0933932334$ $F,-0.3442365784,1.2613302447,-2.5454638865$ $F,-1.1399666166,-0.75613303,-2.5807207158$
$H F=-568.8289783 \quad N I m a g=0$

4- $\mathrm{CF}_{3}-\mathrm{C}_{6} \mathrm{H}_{4}^{-}$

Brutoformula: C7H4F3(1-)

$-1,1$

$\mathrm{H},-2.1497227563,-0.0329827149,-0.2919796532$

C,-1.204880257,-0.018587367,0.2476954495

C, $1.1775066517,0.0076498309,1.6443297542$

C, $-0.0000000005,-0.0371747613,-0.4677805392$

C, $-1.1775066518,0.0076498272,1.6443297548$

$\mathrm{C}, 0.0000000001,0.022466538,2.437176587$

C, $1.2048802562,-0.0185873635,0.2476954491$

C, $0.0000000001,0.0009764102,-1.9493617352$

$\mathrm{H},-2.151561082,0.0137837419,2.1422133564$

$F, 1.089917204,-0.6006642446,-2.5133298827$

$\mathrm{H}, 2.1497227554,-0.0329827086,-0.291979654$

$\mathrm{H}, 2.1515610822,0.0137837487,2.1422133551$

$\mathrm{F}, 0.0000000104,1.2753332907,-2.4778923553$

$\mathrm{F},-1.0899172123,-0.6006642277,-2.5133298836$

$H F=-568.8298446 \quad$ NImag $=0$

\section{1,3- $\left(\mathrm{CF}_{3}\right)_{2}-\mathrm{C}_{6} \mathrm{H}_{4}$}

Brutoformula: C8H4F6

0,1

$\mathrm{H}, 0.0000000003,-0.0347766676,3.0649395741$

C, $0.0000000001,-0.0171375781,1.9819448509$

C, $-0.0000000002,0.0440027672,-0.8099007448$

$\mathrm{C}, 1.2065456897,-0.0024423765,1.2885069185$

C,-1.2065456898,-0.0024423769,1.2885069188

C,-1.2022419423,0.0304963875,-0.1060793725

C, $1.2022419419,0.0304963877,-0.1060793729$

$\mathrm{H}, 2.1464386191,-0.0053904834,1.8258776162$

$\mathrm{H},-2.1464386189,-0.0053904841,1.825877617$

C,-2.507973656,-0.0003472904,-0.8578035208

C, $2.5079736559,-0.000347291,-0.8578035207$

$\mathrm{H},-0.0000000004,0.07990684,-1.8912589385$

$\mathrm{F},-2.9185203062,-1.2719581887,-1.0905498233$

$\mathrm{F},-2.41930826,0.6059091668,-2.0604977435$

F,-3.501046978,0.6077351067,-0.1723164464

F,2.9185203082,-1.2719581895,-1.0905498185

$\mathrm{F}, 3.5010469765,0.60773511,-0.1723164477$

$F, 2.41930826,0.6059091612,-2.0604977459$

$\mathrm{HF}=-906.6077955 \quad \mathrm{NImag}=0$

1,3- $\left(\mathrm{CF}_{3}\right)_{2}-\mathrm{C}_{6} \mathrm{H}_{3}^{-}$

Brutoformula: C8H3F6(1-)

$-1,1$

$F,-2.38750898,-0.2627506222,-2.0026592649$

C, $-0.0000000104,0.1966289648,2.2453544721$

C, $0.0000000133,-0.0703434323,-0.6633967854$

C, $-1.1736076634,0.1248427443,1.4593336584$

C, $1.1736076617,0.1248423213,1.4593337149$

C, $1.1915677662,-0.0047391976,0.0583870911$

C,-1.1915677453,-0.0047388451,0.0583870376 
$\mathrm{H},-2.1451793314,0.170137466,1.9548206147$ $\mathrm{H}, 2.1451793156,0.1701365937,1.9548207359$ C, $2.5004752517,-0.055483553,-0.6588954351$ C, $-2.5004752472,-0.05548334,-0.6588954517$ $\mathrm{H}, 0.0000000221,-0.1706507429,-1.7395755334$ $F, 3.2285926464,1.0960300316,-0.5286580164$ $F, 2.3875090412,-0.2627529426,-2.0026589329$ $F, 3.3246308658,-1.0458517306,-0.2035201418$ F,-3.2285937258,1.0960293157,-0.528656187 $F,-3.3246298806,-1.045853031,-0.2035215752$

$\mathrm{HF}=-905.9940098 \mathrm{NImag}=0$

\section{$\mathrm{C}_{6}\left(\mathrm{CF}_{3}\right)_{5} \mathrm{CH}_{3}$}

Brutoformula: $\mathrm{C} 12 \mathrm{H} 3 \mathrm{~F} 15$

0,1

C, $1.4972770355,2.5024046703,0.2284047753$ C, $0.681766181,1.2116132747,0.0392393226$ C, $-0.7226330588,-1.2178860739,-0.0035358186$ C, $-0.7243726213,1.2192096863,-0.002506847$ C, $1.4025267843,0.0022011648,-0.0075122834$ C, $0.683521771,-1.2083685911,0.0381853337$ C,-1.4291517709,0.0001333862,0.0743613449 C,-1.4803378712,2.5263956783,-0.3403602446 C, $2.906123872,0.0036628654,-0.2260590528$ C, $1.5006471004,-2.4982375607,0.2268629295$ C,-2.9294776808,-0.0010532245,0.4497476775 C,-1.47668306,-2.5258474359,-0.3426878721 $\mathrm{F}, 2.0251768822,2.9587419211,-0.9256459506$ $F, 2.5265736657,2.2806975958,1.0780350084$ F, $0.7897758014,3.4922867202,0.7858200725$ $F,-2.6186260967,2.2582783241,-1.0038234386$ F, $-0.7387015505,3.2667615498,-1.1914818278$ $F,-1.7899119291,3.284275289,0.7166348278$ F,-3.7863110307,-0.0008816486,-0.57548305 $\mathrm{F},-3.2041493724,1.0719065236,1.2190383136$ F,-3.2028245322,-1.0753676203,1.2176420673 $\mathrm{F},-2.6150684377,-2.2588469406,-1.0064224083$ F,-1.7855982565,-3.2849944956,0.7136019267 F,-0.7336447722,-3.2644900803,-1.1940916694 F,2.0288830399,-2.9536347701,-0.9274034914 F,0.7944169968,-3.4891518056,0.7840267198 $\mathrm{F}, 2.5297486226,-2.2756254291,1.0765143395$ $\mathrm{H}, 3.2060327374,0.8798416286,-0.7954037188$ $\mathrm{H}, 3.2076717319,-0.8708474121,-0.7970523789$ $\mathrm{H}, 3.4590897591,0.0033249992,0.712786313$

$H F=-1957.2912742 \quad N I m a g=0$

$\mathrm{C}_{6}\left(\mathrm{CF}_{3}\right)_{5} \mathrm{CH}_{2}{ }^{-}$

Brutoformula: C12H2F15(1-)

$-1,1$

C, $1.4929414314,2.4377750101,0.4955241828$ C, $0.6784854325,1.2286242425,0.1170760508$ C, $-0.7043761222,-1.2275969627,0.0114129823$ C, $-0.7043523352,1.2276150912,0.0113796842$
C, $1.4433481527,-0.000020097,-0.1482670826$ C, $0.6784567192,-1.2286428732,0.1170748421$ C,-1.4454849163,0.0000160026,-0.033897981 C, $-1.4245775302,2.5616193651,-0.2431405533$ C, $2.7079356859,-0.0000222274,-0.6322622116$ C, $1.4928786027,-2.4378298113,0.4954601949$ C, $-2.9290413334,0.0000499011,0.2220755359$ C,-1.4246030968,-2.5615943068,-0.2430880907 $F, 2.0986809845,3.0726631321,-0.5558488692$ $F, 2.5035075665,2.0999216376,1.3412231483$ $F, 0.7962510491,3.3921472194,1.1588136366$ F,-2.5069171471,2.3948658828,-1.038952217 F,- $0.6339212156,3.4225081886,-0.9337889108$ $\mathrm{F},-1.8413416353,3.2221863797,0.8580949437$ F,-3.7355843391,0.0000789618,-0.8797057055 F,-3.3269352141,1.0730903898,0.9608081549 F,-3.3269905244,-1.0729887944,0.9607797607 $\mathrm{F},-2.5069347136,-2.3948571176,-1.0389145723$ $F,-1.8413547897,-3.2221219425,0.8581735864$ $F,-0.6339082371,-3.4224965226,-0.9336825983$ $F, 2.098335806,-3.0728486359,-0.5559921735$ $F, 0.7962279624,-3.392097256,1.1589408938$ $\mathrm{F}, 2.5036487794,-2.0999941289,1.3409254999$ $\mathrm{H}, 3.2417467686,0.9196196466,-0.8217212225$ $\mathrm{H}, 3.2414022623,-0.9196703746,-0.8226580109$

$H F=-1956.7593508 \quad$ NImag $=0$

\section{$\mathrm{C}_{6} \mathrm{H}_{5} \mathrm{CH}_{3}$}

Brutoformula: $\mathrm{C} 7 \mathrm{H} 8$

0,1

C, $0 ., 0.003183114,-2.2310797939$

C, $0 .,-0.0149075701,-0.7202570315$

C, $0 ., 0.0099221401,2.0950511048$

C, $1.2005168143,-0.0110668652,-0.0016926703$ C,-1.2005168144, $-0.0110668651,-0.0016926703$ C, $-1.2035221489,0.0021503396,1.3920608031$ C, $1.2035221489,0.0021503396,1.392060803$ $\mathrm{H}, 2.1436074931,-0.0210291815,-0.5391602201$ $\mathrm{H},-2.1436074932,-0.0210291813,-0.53916022$ $\mathrm{H},-2.1460931881,0.0026694266,1.928479767$ $\mathrm{H}, 2.1460931881,0.0026694269,1.928479767$ $\mathrm{H}, 0.0000000001,0.0168001191,3.1791641576$ $\mathrm{H}, 0.0000000001,1.0304461996,-2.6111045028$ $\mathrm{H},-0.8840409767,-0.4954457221,-2.6355746473$ $\mathrm{H}, 0.8840409767,-0.4954457222,-2.6355746471$

$H F=-271.6388128 \quad \mathrm{~N} I \mathrm{mag}=0$

\section{$\mathrm{C}_{6} \mathrm{H}_{5} \mathrm{CH}_{2}^{-}$}

Brutoformula: $\mathrm{C} 7 \mathrm{H} 7(1-)$

$-1,1$

C,-0.1468386991,-0.0000233103,-2.3818210424 C, $-0.1048015905,-0.000008985,-0.9916747892$ C, $-0.0182329751,0.0000210941,1.921945468$ C, $-0.0790628418,1.2111489335,-0.1983067285$ 
C, $-0.0829749883,-1.2111629821,-0.1981515512$ C, $-0.0419961655,-1.1991846384,1.1834572155$ C, $-0.0381408796,1.1991961941,1.1833102166$ $\mathrm{H},-0.0931912952,2.1649348342,-0.7221400249$ $\mathrm{H},-0.1002382258,-2.1649532209,-0.721882698$ $\mathrm{H},-0.0278513805,-2.1513760814,1.7134658994$ $\mathrm{H},-0.0209171221,2.1514045906,1.7131973622$ $\mathrm{H}, 0.0131779716,0.0000387604,3.0062332084$ $\mathrm{H},-0.1667278825,-0.9278604974,-2.9445254226$ $\mathrm{H},-0.1633153805,0.9278253086,-2.9446307884$

$H F=-271.0178845 \quad N I m a g=0$

\section{2- $\mathrm{CF}_{3}-\mathrm{C}_{6} \mathrm{H}_{3} \mathrm{CH}_{3}$}

Brutoformula: $\mathrm{C} 8 \mathrm{H} 7 \mathrm{~F} 3$

0,1

C,2.1111938985,0.0000000025,0.3624761777 C, $0.7190390048,-0.0000000066,-0.2249460524$ $\mathrm{C},-1.8440216581,0.0000000004,-1.4154434692$ C, $-0.4502600341,-0.0000000073,0.5628720966$ C, $0.5579366563,-0.0000000086,-1.6131812693$ C,- $0.7019281305,-0.0000000052,-2.2081841386$ C,-1.7137482453,-0.0000000042,-0.0296312047 C, $-0.3594093258,-0.0000000032,2.0683415287$ $\mathrm{H}, 1.443492871,0.0000000032,-2.2394772269$ $\mathrm{H},-0.7867613557,0.0000000022,-3.2890644837$ $\mathrm{H},-2.5958113133,0.000000002,0.5966973692$ $\mathrm{H},-2.8294121732,0.0000000109,-1.865861931$ $\mathrm{H}, 2.2820056595,0.8791853931,0.9878989489$ $\mathrm{H}, 2.8562775795,0.0000000053,-0.4341105005$ $\mathrm{H}, 2.2820056695,-0.879185383,0.9878989532$ $F, 0.3011628933,1.0865412942,2.5457408034$ $\mathrm{F}, 0.3011628837,-1.0865413029,2.5457408118$ $\mathrm{F},-1.5729251411,0.000000007,2.6622339537$

$H F=-608.7867018 \quad \mathrm{~N} I m a g=0$

\section{2- $\mathrm{CF}_{3}-\mathrm{C}_{6} \mathrm{H}_{3} \mathrm{CH}_{2}^{-}$}

Brutoformula: $\mathrm{C} 8 \mathrm{H} 6 \mathrm{~F} 3(1-)$

$-1,1$

$F, 0.2872466712,1.0662215028,2.7207207087$ $\mathrm{C}, 0.9315861692,0.0465277734,-0.0878837554$ C,-1.6934446044,-0.0852137178,-1.3578358276 C, $-0.3213317874,-0.0299978575,0.6681495961$ C, $0.7303463187,0.0482128582,-1.5297361983$ C, $-0.5012404493,-0.0134970347,-2.1243208229$ C, $-1.5628356175,-0.0914686934,0.0249976542$ C, $-0.293170838,-0.0446105666,2.1420765773$ $\mathrm{H}, 1.6223492548,0.1021214845,-2.1490732616$ $\mathrm{H},-0.5627892863,-0.0073480334,-3.2112765664$ $\mathrm{H},-2.4557620943,-0.1465339165,0.6379267189$ $\mathrm{H},-2.6683318179,-0.1341887924,-1.828812535$ F,-1.5362987295,-0.1181062584,2.7179178815 $\mathrm{F}, 0.4042737378,-1.0993243288,2.6963021371$ C,2.2023635428,0.1120323242,0.4402521466 $\mathrm{H}, 2.3998452119,0.1139189406,1.5026683644$
$H, 3.0579000561,0.1659425907,-0.2249703834$

$H F=-608.188289 \quad N I m a g=0$

$3-\mathrm{CF}_{3}-\mathrm{C}_{6} \mathrm{H}_{3} \mathrm{CH}_{3}$

Brutoformula: $\mathrm{C} 8 \mathrm{H} 7 \mathrm{~F} 3$

0,1

C,-0.4964222395, $0.0002583904,-2.5874815746$

C, $-0.5105621996,-0.0339360997,-1.0836796821$ C, $-0.4998547186,-0.0067522396,1.6880139002$ C, $0.7004002111,-0.0252870522,-0.389838753$

C, $-1.7222579254,-0.0252898693,-0.3952392198$ C, $-1.70842031,-0.0141828353,0.9971019521$ C, $0.7228685861,-0.0094703614,1.0063162763$ $\mathrm{H}, 1.6330053778,-0.0402445453,-0.9423164663$ $\mathrm{H},-2.658445462,-0.0388247679,-0.9380755732$ $\mathrm{H},-2.6441411693,-0.0169944804,1.5441019879$ C,2.0312199713,0.0262527297,1.758038612 $\mathrm{H},-0.5039587112,-0.0036026505,2.7732191843$ $\mathrm{H}, 2.3270531864,1.058118368,1.9757815152$ $\mathrm{H}, 2.8364488469,-0.4308324439,1.1791966758$ $\mathrm{H}, 1.9563327371,-0.5007032759,2.7119430871$ F, $-0.336826483,1.2636286465,-3.0625497904$ $F,-1.6432193572,-0.4704157472,-3.1264841096$ F, $0.5167796581,-0.7317217671,-3.1080480219$

$H F=-608.7889021 \quad \mathrm{~N} I \mathrm{mag}=0$

3- $\mathrm{CF}_{3}-\mathrm{C}_{6} \mathrm{H}_{3} \mathrm{CH}_{2}^{-}$

Brutoformula: $\mathrm{C} 8 \mathrm{H} 6 \mathrm{~F} 3(1-)$

$-1,1$

C, $0.3838507311,-0.1670137559,-2.4793823564$ C, $0.3437519797,-0.0352563673,-0.9922167667$ C, $0.2768356553,0.0817482585,1.7787784481$ C, $-0.880250101,0.0281012724,-0.3514505623$ C, $1.5676644042,-0.0441663825,-0.2902284803$ C, $1.4834086278,0.0157328172,1.1120502774$ C, $-0.9974298182,0.0974448339,1.089750593$ $\mathrm{H},-1.7902875811,0.0316200813,-0.9406366569$ $\mathrm{H}, 2.5172597745,-0.0863015732,-0.8066330458$ $\mathrm{H}, 2.4034829333,0.0124485855,1.6936776126$ F,0.651887235,-1.4467126486,-2.9031213331 $\mathrm{H}, 0.2701122086,0.126970874,2.8651831601$ F,- $0.7750703229,0.1767719457,-3.1049221176$ F,1.3567595759,0.5982932977,-3.0633213846 C,-2.2197330098,0.1729475845,1.7384894099 $\mathrm{H},-3.1516593047,0.1808977912,1.1837531169$ $\mathrm{H},-2.2787663797,0.2226535666,2.8204574537$

$\mathrm{HF}=-608.1864037 \mathrm{NImag}=0$

\section{4- $\mathrm{CF}_{3}-\mathrm{C}_{6} \mathrm{H}_{3} \mathrm{CH}_{3}$}

Brutoformula: $\mathrm{C} 8 \mathrm{H} 6 \mathrm{~F} 3(1-)$

$-1,1$

C, $-0.015346171,0.0000300529,-2.6487255472$

C, $0.0317380823,-0.0000878763,-1.1954992928$ 
C, $-0.1003776938,-0.000005729,1.7074168282$ C, $-0.0126599607,1.209413327,-0.4552244703$ C, $-0.0160511173,-1.2095067941,-0.4553053885$ C, $-0.0725992306,-1.2167152808,0.914964496$ C, $-0.069172352,1.2166815243,0.9150449059$ $\mathrm{H}, 0.0097019428,2.1548944413,-0.9895390096$ $\mathrm{H}, 0.0036106431,-2.1550102679,-0.9896850263$ $\mathrm{H},-0.0930960565,-2.1682084205,1.440169584$ $\mathrm{H},-0.086927952,2.1681890445,1.4403223233$ $\mathrm{F},-1.3018023328,0.002003563,-3.2218429261$ $\mathrm{F}, 0.5749913502,1.0973572628,-3.2281597561$ $F, 0.5716247705,-1.0990656549,-3.2282071016$ C, $-0.1483037369,0.0000002269,3.0851972204$ $\mathrm{H},-0.1677551352,0.9270367559,3.6484167189$ $\mathrm{H},-0.1703999081,-0.9270061749,3.6483677829$

$\mathrm{HF}=-608.1959799 \mathrm{NImag}=0$

\section{4- $\mathrm{CF}_{3}-\mathrm{C}_{6} \mathrm{H}_{3} \mathrm{CH}_{2}^{-}$}

Brutoformula: $\mathrm{C} 8 \mathrm{H} 7 \mathrm{~F} 3$

0,1

C,-0.4964222395, $0.0002583904,-2.5874815746$ C, $-0.5105621996,-0.0339360997,-1.0836796821$ C, $-0.4998547186,-0.0067522396,1.6880139002$ $\mathrm{C}, 0.7004002111,-0.0252870522,-0.389838753$ C, $-1.7222579254,-0.0252898693,-0.3952392198$ C, $-1.70842031,-0.0141828353,0.9971019521$ C, $0.7228685861,-0.0094703614,1.0063162763$ $\mathrm{H}, 1.6330053778,-0.0402445453,-0.9423164663$ $\mathrm{H},-2.658445462,-0.0388247679,-0.9380755732$ $\mathrm{H},-2.6441411693,-0.0169944804,1.5441019879$ C,2.0312199713,0.0262527297,1.758038612 $\mathrm{H},-0.5039587112,-0.0036026505,2.7732191843$ $\mathrm{H}, 2.3270531864,1.058118368,1.9757815152$ $\mathrm{H}, 2.8364488469,-0.4308324439,1.1791966758$ $\mathrm{H}, 1.9563327371,-0.5007032759,2.7119430871$ F,-0.336826483,1.2636286465,-3.0625497904 F,-1.6432193572,-0.4704157472,-3.1264841096 $F, 0.5167796581,-0.7317217671,-3.1080480219$

$H F=-608.7889021 \quad \mathrm{NImag}=0$

\section{3,5- $\left(\mathrm{CF}_{3}\right)_{2}-\mathrm{C}_{6} \mathrm{H}_{3} \mathrm{CH}_{3}$}

Brutoformula: $\mathrm{C} 9 \mathrm{H} 6 \mathrm{~F} 6$

0,1

C, $0 ., 0.0183859087,3.633317236$

C, $0 .,-0.017124955,2.1249755767$

C, $0 .,-0.0424198218,-0.6958459145$

C, $-1.1990570705,-0.0253887116,1.4080026682$ C, $1.1990570705,-0.0253887116,1.4080026682$ C, $1.1977416172,-0.0392001264,0.0141137667$ C,-1.1977416172,-0.0392001264,0.0141137667 $\mathrm{H},-2.143080868,-0.0345112715,1.9399884283$ $\mathrm{H}, 2.143080868,-0.0345112715,1.9399884283$ C,2.5071120377,-0.0017737832,-0.7306007286 C,-2.5071120377,-0.0017737832,-0.7306007286
$\mathrm{H}, 0 .,-0.0664224759,-1.7771590119$ $\mathrm{H}, 0 ., 1.0516771506,3.9957873797$ $\mathrm{H}, 0.8845506677,-0.4735779651,4.0426316719$ $\mathrm{H},-0.8845506677,-0.4735779651,4.0426316719$ F,-2.9241074706,1.272114812,-0.9418982918 F,-3.4964524211,-0.6238682824,-0.0511945389 $F,-2.4229849803,-0.5885671707,-1.9433926378$ F, 2.9241074706,1.272114812,-0.9418982918 $F, 2.4229849803,-0.5885671707,-1.9433926378$ $F, 3.4964524211,-0.6238682824,-0.0511945389$

$\mathrm{HF}=-945.9364115 \quad \mathrm{NImag}=0$

\section{3,5- $\left(\mathrm{CF}_{3}\right)_{2}-\mathrm{C}_{6} \mathrm{H}_{3} \mathrm{CH}_{2}$}

Brutoformula: C9H5F6(1-)

$-1,1$

$\mathrm{F},-2.8484729284,1.2650213826,1.02167322$

C,-0.0016051711,-0.0650517712,-2.2409967767 C, $0.0045209178,-0.046643176,0.6760862736$ C, $1.2139067379,-0.0328337077,-1.4532035576$ C,-1.2131640165,-0.0770164373,-1.4481683607 C, $-1.1906883004,-0.0707418222,-0.0674868447$ C, $1.1980373361,-0.0282426146,-0.0734894842$ $\mathrm{H}, 2.1628589389,-0.019145567,-1.9758756863$ $\mathrm{H},-2.1653004493,-0.0988977806,-1.9654229826$ C,-2.4865465864,-0.0122382516,0.6787662878 C, $2.4890561888,0.0446065882,0.6795890146$ $\mathrm{H}, 0.0067941672,-0.0630248537,1.7553849422$ F,-3.54620386,-0.5085213238,-0.0157977302 $\mathrm{F},-2.4596722231,-0.6990523911,1.8570105464$ C, $-0.0019957101,-0.0845017309,-3.6219772873$ $F, 2.7042125836,1.2703552707,1.2535723762$ $F, 3.5903188828,-0.1958414342,-0.0795237062$ $\mathrm{F}, 2.552132895,-0.8431756069,1.716192574$ $\mathrm{H},-0.9293052886,-0.107444071,-4.1830230448$ $\mathrm{H}, 0.9249861055,-0.0745832645,-4.1839243383$

$\mathrm{HF}=-945.3514747 \mathrm{NImag}=0$

\section{$\mathrm{C}_{6}\left(\mathrm{CF}_{3}\right)_{5} \mathrm{NH}_{2}$}

Brutoformula: $\mathrm{C} 11 \mathrm{H} 2 \mathrm{~F} 15 \mathrm{~N} 1$

0,1

C,-0.1810472777,-0.0918311455,-1.6899502648 C, $-0.0149249858,-0.1574671676,-0.1717157991$ C, $0.0634624455,-0.1497216289,2.6414682595$ C, $0.0616245669,-1.3648838273,0.5341344819$ C, $0.026256218,1.0861865333,0.5247017899$ C, $-0.0093233481,1.066741983,1.9500051846$ C, $-0.0023680229,-1.3761352748,1.9445940139$ C, $0.4194418902,-2.668085222,-0.2207956703$ $\mathrm{N}, 0.1927020303,2.2534682979,-0.1512856703$ C,- $0.1571474871,2.4137252284,2.6581246009$ C, $-0.3784251394,-2.6698951552,2.6889828259$ $\mathrm{C}, 0.4193748351,-0.1521103848,4.1481372453$ $\mathrm{F}, 0.9676925823,0.2406806274,-2.3207884037$ $\mathrm{F},-1.0862224656,0.8771679344,-2.0207424995$ 
F,- $0.6568721413,-1.2076292584,-2.2399442524$ $F, 1.0727404061,-3.5234330488,0.5823470122$ F,1.2869807228,-2.3866730103,-1.2182824714 F, $-0.6281358701,-3.31482975,-0.7446113848$ $\mathrm{F}, 0.6389958078,-3.4239127684,3.1224837416$ F,-1.148959581,-3.444853367,1.8941546002 $\mathrm{F},-1.14928978,-2.3735972766,3.758260506$ $\mathrm{F}, 1.0677008436,-1.278560937,4.4858844324$ F,-0.6296606029,-0.0201844973,4.9680135152 $F, 1.2899585983,0.8483741156,4.4078406284$ $F, 1.0043437602,3.1017569193,2.7072738095$ $F,-0.6524085732,2.330494861,3.8921277429$ $\mathrm{F},-1.038349806,3.2096033402,1.9802940027$ $\mathrm{H}, 0.0766981547,3.136104583,0.3142943284$ $\mathrm{H}, 0.0992872794,2.2836771664,-1.1510757021$

$H F=-1973.3475187 \quad \mathrm{NImag}=0$

\section{$\mathrm{C}_{6}\left(\mathrm{CF}_{3}\right)_{5} \mathrm{NH}^{-}$}

Brutoformula: C11H1F15N1(1-)

$-1,1$

C,-0.2559403298,0.1006558567,-1.6107511372 C, $0.0629988184,-0.1621375546,-0.1590155202$ C, $-0.0027071611,-0.157798745,2.6408426841$ C, $-0.037154433,-1.3595348092,0.5171860314$ C, $0.6184128095,0.9928222098,0.5770850423$ C, $0.0970690968,1.0503822705,1.9628884024$ C, $0.1681921849,-1.3902485734,1.940829487$ C, $-0.5252617238,-2.6195565496,-0.195354014$ $\mathrm{N}, 1.4328202021,1.7939840176,-0.0086587643$ C, $-0.3368767153,2.3828989099,2.5100817749$ C, $0.8378230601,-2.5575960696,2.6126869999$ C, $-0.3738784513,-0.1981936966,4.1247043947$ F, $0.8197933701,-0.0573754589,-2.426664857$ F,- $0.7019108338,1.3656617946,-1.8040042923$ F,-1.2289474109,-0.6953556985,-2.1215019037 $F,-0.3694431563,-3.7516279513,0.5156650235$ $F, 0.0993916079,-2.8324568207,-1.3767952157$ $F,-1.8583268168,-2.5450400986,-0.4484967344$ $F, 1.7409291901,-3.1611538369,1.7854762579$ $F, 0.0421852919,-3.559582053,3.0796263517$ $\mathrm{F}, 1.5731239154,-2.1538849791,3.6885419494$ $F, 0.6380972264,0.1764445783,4.9421173765$ F, $-0.7715745521,-1.4219784393,4.5314398659$ F,-1.419275873,0.6189436199,4.4015084207 F, $0.1263428678,2.6491397383,3.7617578391$ F,-1.6925685431,2.5219072969,2.5701503145 F,0.0836273993,3.4268427351,1.7479650854 $\mathrm{H}, 1.7101231074,2.5539516966,0.6077725283$

$H F=-1972.8245158 \quad \mathrm{NImag}=0$

\section{$\mathrm{C}_{6} \mathrm{H}_{5} \mathrm{NH}_{2}$}

Brutoformula: $\mathrm{C} 6 \mathrm{H} 7 \mathrm{~N} 1$

0,1

$\mathrm{N}, 0.0433114052,-0.5965912404,-2.3795754306$
C, $0.0602492296,-0.5965910453,-0.9814730932$ C,- $0.0217188953,-0.5965906351,1.834030693$ C, $0.0383336748,-1.8027893694,-0.2653638943$ C, $0.0383336415,0.6096074979,-0.2653642464$ C,- $0.0015817179,0.6042547407,1.1252637207$ C,-0.0015816832,-1.7974362106,1.1252640767 $\mathrm{H}, 0.0482044708,-2.7450091363,-0.8045913728$ $\mathrm{H}, 0.048204419,1.5518270998,-0.8045920123$ $\mathrm{H},-0.0164781714,1.5488868831,1.6581389078$ $\mathrm{H},-0.0164781078,-2.7420682018,1.6581395314$ $\mathrm{H},-0.0520356956,-0.5965904757,2.9169426279$ $\mathrm{H}, 0.3936794995,0.2408985801,-2.8207274121$ $\mathrm{H}, 0.3936795489,-1.434081164,-2.8207271768$

$H F=-287.6876264 \quad N I m a g=0$

\section{$\mathrm{C}_{6} \mathrm{H}_{5} \mathrm{NH}^{-}$}

Brutoformula: $\mathrm{C} 6 \mathrm{H} 6 \mathrm{~N} 1(1-)$

$-1,1$

$\mathrm{N}, 0.1306467677,-0.5259035237,-2.3899986645$ C, $0.0811911578,-0.5942984028,-1.0604198804$ C, $-0.0278202288,-0.5996036238,1.8485049566$ C, $0.0665870025,-1.806709332,-0.2685534632$ C, $0.035748101,0.6150601405,-0.2688827947$ C, $-0.0157116505,0.6009714338,1.1127885991$ C, $0.0146608374,-1.7966488343,1.1171638334$ $\mathrm{H}, 0.0985930102,-2.7587493474,-0.7972759823$ $\mathrm{H}, 0.0439851948,1.5574424705,-0.8097937887$ $\mathrm{H},-0.0478831262,1.5511811294,1.6451295062$ $\mathrm{H}, 0.0069775819,-2.7480942826,1.6487262895$ $\mathrm{H},-0.0675372383,-0.5993787681,2.9327823431$ $\mathrm{H}, 0.1576233515,-1.4772453576,-2.7645339332$

$\mathrm{HF}=-287.0901751 \quad \mathrm{NImag}=0$

\section{2- $\mathrm{CF}_{3}-\mathrm{C}_{6} \mathrm{H}_{4} \mathrm{NH}_{2}$}

Brutoformula: C7H6F3N1

0,1

$\mathrm{H},-0.0240530388,0.3325342668,-3.2918386851$

C,- $-0.0197354601,0.3472441157,-2.2076152216$ C,- $0.009381933,0.3516211596,0.5776136053$ C, $0.0662898766,-0.8501012889,-1.5144710591$ C, $-0.1048605378,1.5608178902,-1.5238320649$ C, $-0.0972057541,1.5501436928,-0.1354814386$ C, $0.0737223694,-0.8821726113,-0.1086833952$ $\mathrm{H}, 0.1325800626,-1.7873081687,-2.0574576783$ $\mathrm{H},-0.1756670304,2.4972213954,-2.0624637311$ $\mathrm{H},-0.1615804148,2.4801982476,0.4138618639$ $\mathrm{N}, 0.214405493,-2.0937605506,0.5463398384$ C, $0.0398019745,0.3930578934,2.0756934749$ $\mathrm{H}, 0.035404873,-2.9187010554,-0.0044434633$ $\mathrm{H},-0.1158868647,-2.1621585522,1.4959324939$ $F, 1.2459618052,0.010252525,2.569916918$ F,- $0.8729476726,-0.4524327264,2.6499632637$ $F,-0.2125785892,1.6177175179,2.5767697213$ 
$\mathrm{HF}=-624.837608 \mathrm{NImag}=0$

\section{$2-\mathrm{CF}_{3}-\mathrm{C}_{6} \mathrm{H}_{4} \mathrm{NH}^{-}$}

Brutoformula: C7H5F3N1(1-)

$-1,1$

$\mathrm{H},-0.1917014884,0.0000000099,-3.2917577167$

C, $-0.1849673997,0.0000000038,-2.2031666981$

C, $-0.1531995495,-0.0000000142,0.5906371051$

C, $1.0177424094,0.0000000073,-1.5447616035$

$\mathrm{C},-1.4148263463,-0.0000000091,-1.5011661123$

C,-1.3646915699,-0.0000000056,-0.1152718684

C, $1.1396617914,-0.0000000249,-0.0970345333$

$\mathrm{H}, 1.9538448359,0.00000002,-2.0944146835$

$\mathrm{H},-2.3639882054,-0.0000000069,-2.0249233554$

$\mathrm{H},-2.2912940624,0 ., 0.448690881$

$\mathrm{N}, 2.3501496123,-0.0000000031,0.4275645499$

C,-0.1935734509,-0.0000000019,2.0654311267

$\mathrm{F},-1.4594503619,0.0000000244,2.5868308689$

$\mathrm{H}, 2.3066987062,0.0000000091,1.4438624163$

F, 0.4247976386, 1.0844268809,2.654739787

$F, 0.4247976106,-1.0844268896,2.6547398093$

$H F=-624.2600258 \quad \mathrm{NImag}=0$

\section{3- $\mathrm{CF}_{3}-\mathrm{C}_{6} \mathrm{H}_{4} \mathrm{NH}_{2}$}

Brutoformula: $\mathrm{C} 7 \mathrm{H} 6 \mathrm{~F} 3 \mathrm{~N} 1$

0,1

$\mathrm{F},-0.8016510149,-1.8257377895,2.7722301905$ C, $0.1418240266,0.448317182,-1.3164897334$

C, $-0.0693236066,0.5364114074,1.4947619333$

C, $0.0095720298,-0.7326120703,-0.5736401041$

C, $0.1640435957,1.6733108581,-0.6308265266$

C, $0.0592513531,1.7096892356,0.754013379$

C, $-0.0946053646,-0.6803268689,0.8138495981$

$\mathrm{H},-0.0242254783,-1.6891128938,-1.0821562399$

$\mathrm{H}, 0.2570543484,2.5976894424,-1.1915935183$

$\mathrm{H}, 0.076504618,2.6663610908,1.2633417728$

C, $-0.1711781461,-1.9697722477,1.5855898428$

$\mathrm{H},-0.1544456282,0.566304579,2.5725558429$

$\mathrm{N}, 0.1874980196,0.4079812765,-2.7072613997$

$F, 1.0639418403,-2.4654282691,1.8637849647$

F,- $0.8248128474,-2.940802902,0.9046475513$

$\mathrm{H}, 0.5896301262,1.2115428491,-3.1656054144$

$\mathrm{H}, 0.4778638033,-0.4648174307,-3.1215577829$

$H F=-624.8381278 \quad N I m a g=0$

\section{$3-\mathrm{CF}_{3}-\mathrm{C}_{6} \mathrm{H}_{4} \mathrm{NH}^{-}$}

Brutoformula: C7H5F3N1(1-)

$-1,1$

$\mathrm{N}, 0.1268103184,-0.5246642599,-2.3879563607$ C, $0.0749959684,-0.5909289599,-1.06326472$

C, $-0.0405254492,-0.5898023168,1.8469262271$ C, $0.0287788462,-1.8038691395,-0.2752298239$ C, $0.0586646133,0.6188325487,-0.270655132$ C, $0.0031622533,0.605907438,1.1083237215$
C, $-0.0281022797,-1.7863708567,1.1121845939$ $\mathrm{H}, 0.0334269814,-2.7548766761,-0.798732917$ $\mathrm{H}, 0.0902912884,1.5596868972,-0.8118962969$ $\mathrm{H},-0.0102277364,1.5534853782,1.6432488257$ C, $0.0071611506,-3.0833200639,1.8534251796$ $\mathrm{H},-0.0916979344,-0.5915190138,2.9273618436$ $\mathrm{H}, 0.1324223888,-1.4736532591,-2.7673077244$ $\mathrm{F}, 1.2792025768,-3.4832488315,2.1848309627$ F, $-0.520567351,-4.1314957507,1.1608759056$ F,-0.6667345097,-3.0493260472,3.0409640334

$H F=-624.2579351 \quad \mathrm{NImag}=0$

\section{4- $\mathrm{CF}_{3}-\mathrm{C}_{6} \mathrm{H}_{4}-\mathrm{NH}_{2}$}

Brutoformula: C7H6F3N1

0,1

$\mathrm{H},-2.1505000723,0.0485807389,-1.4518822028$

C,-1.2075051893,0.0336480765,-0.9156772871 C, $1.2048829978,-0.0022715298,0.4705754059$ C, $0.0000000001,0.0520631675,-1.6336081974$ C, $-1.2048829981,-0.0022715289,0.4705754058$ C, $-0.0000000002,-0.0239731438,1.1765258104$ C, $1.2075051893,0.0336480758,-0.9156772869$ $\mathrm{N}, 0.0000000005,0.1373453449,-3.0182567783$ $\mathrm{H},-2.1455966753,-0.0225671696,1.0069724607$ C, $0.0000000001,-0.0084947271,2.6727944277$ $\mathrm{H}, 2.1505000724,0.0485807379,-1.4518822025$ $\mathrm{H}, 2.1455966749,-0.0225671712,1.0069724609$ $\mathrm{H},-0.8432500288,-0.1433194173,-3.4946068004$ $\mathrm{H}, 0.8432500283,-0.1433194216,-3.4946068004$ $\mathrm{F},-0.0000000044,1.256848515,3.1801476341$ $F, 1.0894051096,-0.6209652706,3.1958169736$ $F,-1.0894051046,-0.6209652785,3.1958169747$

$\mathrm{HF}=-624.8398366 \quad \mathrm{NImag}=0$

\section{4- $\mathrm{CF}_{3}-\mathrm{C}_{6} \mathrm{H}_{4}-\mathrm{NH}^{-}$}

Brutoformula: C7H5F3N1(1-)

$-1,1$

$\mathrm{H},-2.2082796713,0.0252329234,-1.6779758449$ C,-1.2679658821,0.007986033,-1.1360581221 C, $1.1584102017,-0.0433426532,0.2381137975$ C, $-0.0536332126,0.0082897908,-1.9286251457$ C, $-1.2580160689,-0.0171873527,0.2363444311$ C, $-0.0474806022,-0.0555325086,0.9716956398$ C, $1.1638343419,-0.0187643917,-1.1379210348$ $\mathrm{N},-0.1227810811,0.0291078751,-3.2484032118$ $\mathrm{H},-2.2016260288,-0.0177555019,0.7744197871$ C,-0.0490839414,0.0067747572,2.4312405141 $\mathrm{H}, 2.1138143709,-0.0233470731,-1.6685721245$ $\mathrm{H}, 2.1032753884,-0.0640654307,0.7734520743$ $\mathrm{F},-1.1203226555,-0.6238221328,3.0141035647$ $\mathrm{H}, 0.8259011595,0.0248690815,-3.629740091$ F,-0.1056613358,1.2943559518,2.9769681297 $F, 1.0696150171,-0.53279937,3.0109576384$ 
$\mathrm{HF}=-624.2657666 \mathrm{NImag}=0$

\section{3,5- $\left(\mathrm{CF}_{3}\right)_{2}-\mathrm{C}_{6} \mathrm{H}_{3} \mathrm{NH}_{2}$}

Brutoformula: C8H5F6N1

0,1

$\mathrm{N},-0.0021150191,0.1933226284,-3.512987697$ C,-0.000827051,0.0737627601,-2.1319912211 C, $0.0008208521,-0.0762902829,0.6879469355$ C, $1.2050044643,0.0497035287,-1.4158659513$ C,-1.2058504273,0.0244547642,-1.4157992808 C,-1.1945658594,-0.0519195692,-0.0275912603 C,1.1953956908,-0.026890892,-0.0276580511 $\mathrm{H}, 2.1486239649,0.0827113142,-1.9473827212$ $\mathrm{H},-2.1499808761,0.0376846269,-1.9472700181$ C,-2.5054331242,-0.053993522,0.7164682891 C, $2.5060514354,-0.0014566294,0.7163434196$ $\mathrm{H}, 0.0016007864,-0.1479175765,1.7662090756$ $\mathrm{H},-0.8427708862,-0.0800577196,-3.9978547286$ $\mathrm{H}, 0.8440122026,-0.0625099111,-3.997914001$ $F, 2.8938063393,1.2671113385,1.0000308924$ F,3.5099095356,-0.5599883239,0.0015443939 F,2.4395836296,-0.6592619671,1.8928763834 $F,-2.9197222643,1.2061611884,1.0001642739$ $\mathrm{F},-2.4251214507,-0.7102504939,1.8930006733$ F,-3.4973731165,-0.6334817505,0.0017177158

$$
\mathrm{HF}=-961.9860086 \mathrm{NImag}=0
$$

\section{$3,5-\left(\mathrm{CF}_{3}\right)_{2}-\mathrm{C}_{6} \mathrm{H}_{3} \mathrm{NH}^{-}$}

Brutoformula: C8H4F6N1(1-)

$-1,1$

$\mathrm{N}, 0.0725784694,0.0062878606,-3.5742821501$ C, $0.0006682517,-0.0102076065,-2.2535240246$ C, $0.0087295313,-0.0562448727,0.6587980905$ C, $1.2164672211,-0.0078705886,-1.4684721743$ C,-1.2090218,-0.0330076381,-1.4606609195 C,-1.1849200994,-0.0565096247,-0.0744212677 C, $1.2027099893,-0.0327595489,-0.0887216715$ $\mathrm{H}, 2.152695347,0.0078510415,-2.0117417439$ $\mathrm{H},-2.1617181091,-0.0398299978,-1.9797231129$ C,-2.4814845467,-0.0064297526,0.6731030789 C,2.493658002,0.0053393058,0.669098441 $\mathrm{H}, 0.0137359318,-0.0900323225,1.7380754249$ $\mathrm{H},-0.8731381966,0.0024810839,-3.9606258257$ $F,-3.5244110511,-0.5578992358,-0.0053227031$ $F, 2.7027532068,1.2027739436,1.3008785239$ F,3.5931778951,-0.1983459709,-0.0979867896 $F, 2.552366268,-0.9306104176,1.6632885519$ F,-2.8787553687,1.2720406907,0.9651573257 F,-2.4362935672,-0.6477748846,1.8742954236

$$
\mathrm{HF}=-961.4217212 \mathrm{NImag}=0
$$

\section{$\mathrm{C}_{6}\left(\mathrm{CF}_{3}\right)_{5} \mathrm{OH}$}

Brutoformula: C11H1F15O1 0,1
C, $0.3056876699,-0.0111909375,-3.0224735622$ C, $0.2096331759,0.0093953223,-1.4950510477$ C, $0.1836392589,0.0406742132,1.3266425815$ C,-1.0139861933,-0.0093734151,-0.7982870804 C, $1.416358028,0.0418422664,-0.7652866749$ C, $1.4044995327,0.0131922119,0.645834545$ C,-1.0274757076,-0.0980765998,0.6076467431 C, $-2.3322582624,0.2387564157,-1.5670926416$ O,2.6258451993,0.1830167648,-1.3229445067 C,2.7807436746,-0.1166961371,1.3198966957 C,-2.3012000691,-0.5625608549,1.3452246216 C, $0.1503549152,0.4141831081,2.8282444118$ F,-0.7011619436,-0.6434964948,-3.6194057911 $F, 1.432081243,-0.6983660569,-3.4183284254$ $F, 0.4254147947,1.2186171868,-3.547770974$ F,-2.8519985372,-0.864698534,-2.1186505165 F,-2.1188612232,1.1415468997,-2.548649727 F,-3.2650514798,0.7885373627,-0.7764507623 F,-3.007023628,-1.3928690621, 0.5478595722 F,-3.1254747619,0.4030782619,1.7623019727 F,-1.9560918734,-1.3012531065,2.4203850196 $F, 0.3389871124,-0.6123958218,3.6623775942$ $\mathrm{F},-1.0188943156,0.9965702499,3.1465353148$ $F, 1.0930577371,1.3473262172,3.0735626461$ $\mathrm{F}, 2.7000257521,-0.508461969,2.5995807888$ $F, 3.4740364212,1.0335612442,1.2784689095$ $F, 3.5105391635,-1.0673831374,0.7015831421$ $\mathrm{H}, 2.6275806876,-0.016780109,-2.2670453701$

$H F=-1993.2143467 \quad \mathrm{NImag}=0$

$\mathrm{C}_{6}\left(\mathrm{CF}_{3}\right)_{5} \mathrm{O}^{-}$

Brutoformula: C11F15O1(1-)

$-1,1$

C, $0.3497727237,-0.1361962051,-2.9968682096$ C, $0.1959813608,0.0069399144,-1.4971565117$ C, $0.1902655109,0.0373764053,1.3319849553$ C, $-1.0114428091,0.0055968068,-0.8074905259$ C, $1.4624772962,0.1981313952,-0.7925716824$ C, $1.4073646231,0.0379111299,0.6595778135$ C, $-1.0407227221,-0.0563484461,0.6171908996$ C, $-2.3175737249,0.2598260772,-1.5795706224$ O,2.4982770105,0.5296022946,-1.3791119025 C,2.7689277301,-0.0739484934,1.3126663815 C,-2.3005627763,-0.5025770899,1.3315393937 C, $0.1657815244,0.327734745,2.8426593248$ F, $-0.7151813534,-0.7423441113,-3.5890901255$ $F, 1.4016978196,-0.9275801635,-3.3047038894$ $\mathrm{F}, 0.5360645697,1.036030567,-3.6522381452$ F,-2.8984187002,-0.8411932433,-2.0978654617 $\mathrm{F},-2.110344817,1.122421414,-2.6028806312$ F,-3.2485113785,0.868595621,-0.809077856 F,-3.0622655536,-1.3037104515,0.5386743693 F,-3.131016236,0.4713204454,1.7822204674 F,-2.0103587405,-1.2737672828,2.4138331429 $F, 0.3180678216,-0.7524959162,3.6351188726$ 
F, $-0.9842013496,0.9324064893,3.2207339791$ $F, 1.137019327,1.2101502582,3.1776839117$ $F, 2.7247871227,-0.6591402898,2.5406516589$ $F, 3.4082790078,1.1105993446,1.4754677668$ $F, 3.592780639,-0.8662933843,0.5912700273$

$H F=-1992.7281817 \quad$ NImag $=0$

$\mathrm{C}_{6}\left(\mathrm{CF}_{3}\right)_{5} \mathrm{O}$.

Brutoformula: C11F15O1(2)

0,2

C, $0.3375840908,-0.1365326264,-3.0331884798$ C, $0.1723110409,-0.0098711339,-1.5116110724$ C, $0.1989070636,0.062034885,1.3513169745$ C, $-1.0243645069,0.0281610778,-0.8252690814$ C, $1.4381180109,0.1013534428,-0.7782784425$ C, $1.4078120227,0.0242886473,0.6863758179$ C,-1.0178787016,-0.0771984019,0.6050589493 C,-2.3460769205,0.3192768309,-1.576143604 $0,2.4827170444,0.3778915317,-1.3700057342$ C, $2.7939610867,-0.0670492176,1.3407597507$ C,-2.2861272416,-0.5945086031,1.3255984906 C, $0.1486483855,0.3884020042,2.8636509444$ F,-0.7503582625,-0.656586122,-3.6239466471 F,1.3467543436,-0.9794830395,-3.3067261622 $F, 0.6033376704,1.0466117962,-3.6038384509$ F,-2.9179741837,-0.7591774728,-2.1162714733 F,-2.109361852,1.2143322594,-2.5528377957 F,-3.2370791194,0.8991658435,-0.7534133559 F,-2.9774859928,-1.395296375,0.4925928597 $F,-3.1113210771,0.3518121296,1.774002762$ $\mathrm{F},-1.9244810888,-1.3655645909,2.3682599856$ $\mathrm{F}, 0.3233291015,-0.6697832615,3.6583071494$ F,-1.0245107653,0.9630745711,3.1816570617 $F, 1.0956201656,1.3006126524,3.1495494265$ $F, 2.7364979059,-0.5702309401,2.5845411025$ $F, 3.3968276909,1.1287936169,1.3954042362$ F,3.571538015,-0.9054816729,0.6371021894

$H F=-1992.5563227 \quad N I m a g=0$

\section{$\mathrm{C}_{6} \mathrm{H}_{5} \mathrm{OH}$}

Brutoformula: $\mathrm{C} 6 \mathrm{H} 6 \mathrm{O} 1$

0,1

O,-0.0326486022,0.000000002,2.5007992696

C, $-0.0203530189,-0.0000000021,1.1304794733$

C, $-0.1185563372,-0.0000000025,-1.6614586116$ C, $1.163634485,-0.0000000022,0.3904917318$ C, $-1.2541014663,-0.0000000012,0.4787350252$ C,-1.2957684357,-0.0000000033,-0.9120204728 C, $1.1088511218,-0.0000000012,-1.0022629833$ $\mathrm{H}, 2.1232504481,0.0000000006,0.8997816649$ $\mathrm{H},-2.1618348135,0 ., 1.0698449317$ $\mathrm{H},-2.2572294004,0.0000000027,-1.4130448029$ $\mathrm{H}, 2.0321489152,0.0000000007,-1.5704112482$ $\mathrm{H},-0.1579554898,0.0000000042,-2.744052335$
$H, 0.870562595,0.0000000023,2.8331183564$

$\mathrm{HF}=-307.5586325 \mathrm{NImag}=0$

$\mathrm{C}_{6} \mathrm{H}_{5} \mathrm{O}^{-}$

Brutoformula: $\mathrm{C} 6 \mathrm{H} 5 \mathrm{O} 1(1-)$

$-1,1$

O,0.,-0.0000000032,2.7266726246

C, $0 .,-0.0000000015,1.457001838$

C, $0 .,-0.0000000003,-1.4487130858$

C, $-1.2119044899,0.0000000024,0.6669168296$

C, $1.2119044899,0.0000000011,0.6669168296$

C, $1.1999087936,0.0000000042,-0.7206132188$

C, $-1.1999087937,0.0000000041,-0.7206132188$

$\mathrm{H},-2.1533988295,-0.0000000003,1.2107994591$

$\mathrm{H}, 2.1533988295,-0.0000000016,1.210799459$

$\mathrm{H}, 2.1485689318,0.0000000026,-1.2575460391$

$\mathrm{H},-2.1485689319,0.0000000006,-1.257546039$

$\mathrm{H}, 0.0000000002,-0.0000000081,-2.5340754375$

$\mathrm{HF}=-306.9939821 \quad \mathrm{NImag}=0$

\section{$2-\mathrm{CF}_{3}-\mathrm{C}_{6} \mathrm{H}_{4} \mathrm{OH}$}

Brutoformula: $\mathrm{C} 7 \mathrm{H} 5 \mathrm{~F} 3 \mathrm{O} 1$

0,1

$\mathrm{H}, 2.3147073368,0.0204218491,-2.0503153918$ C, $1.3755713006,0.0115361681,-1.5115532564$ C,- $-1.0566816366,-0.0016598384,-0.1110195274$ C, $1.3693595233,0.032221155,-0.1241880907$ C, $0.1610179082,-0.0220194153,-2.1993469721$

C,-1.0419801334,-0.0256610633,-1.5082217032 C, $0.1645954561,0.0242072882,0.5877617548$ $\mathrm{H}, 2.3015618972,0.0554540693,0.4252223757$ $\mathrm{H}, 0.1511424,-0.0393134322,-3.2832038324$ $\mathrm{H},-1.9924100211,-0.0428691312,-2.027110949$ C, $0.1986213302,-0.0311032538,2.0851744947$ O,-2.2819120523,-0.0173828887,0.4786305973 F,-0.082793259,-1.269589318,2.5650996351 F, $-0.7398554867,0.7971133924,2.6504742479$ $F, 1.3858953664,0.3275950146,2.6008806759$ $\mathrm{H},-2.2216204807,0.1744839191,1.4215405857$

$H F=-644.7075163 \quad \mathrm{NImag}=0$

\section{$2-\mathrm{CF}_{3}-\mathrm{C}_{6} \mathrm{H}_{4} \mathrm{O}$}

Brutoformula: C7H4F3O1(1-)

$-1,1$

O,-2.2420772179,-0.0863462809,0.5139420305

C,-1.1392681749,-0.0463476067,-0.0886250526

C, $1.3886441996,0.0441911682,-1.5163340438$ C, $0.1540509306,-0.0018271606,0.5845840027$ C, $-1.0407390434,-0.0406259285,-1.5355603058$ C, $0.1624086082,0.0024727903,-2.207419108$ C, $1.3568463085,0.0412693393,-0.1252166683$ C, $0.1768417876,-0.0012419827,2.0699218896$ $\mathrm{H},-1.9816893547,-0.0727103641,-2.0771904851$ 
$\mathrm{H}, 0.1637165693,0.0043146095,-3.2964493321$ $\mathrm{H}, 2.289166362,0.0734429775,0.4285495412$ $\mathrm{H}, 2.3326621655,0.0780772955,-2.0488757332$ F,-0.4593772861,1.0625902135,2.643636867 $F, 1.4567098557,0.0435905884,2.5828666257$ F,-0.3831145741,-1.1072934107,2.6439507751

$\mathrm{HF}=-644.1618805 \mathrm{NImag}=0$

\section{$3-\mathrm{CF}_{3}-\mathrm{C}_{6} \mathrm{H}_{4} \mathrm{OH}$}

Brutoformula: $\mathrm{C} 7 \mathrm{H} 5 \mathrm{~F} 3 \mathrm{O} 1$

0,1

$\mathrm{H},-0.4799293323,-0.1560033175,-2.3743730611$

C,- $0.5056785897,-0.0913921972,-1.2948701496$

C, $-0.5559952571,0.0603346644,1.4973210171$

C,0.681731254,-0.0462166479,-0.5616559754

C, $-1.7183264924,-0.062287459,-0.613628013$

C,-1.750791046,0.0142068381,0.7767051821

C, $0.6671570972,0.0311512216,0.8277081677$

C,2.0033153406,-0.0183988551,-1.2831587713

$\mathrm{H},-2.648990457,-0.1015827784,-1.1672698452$

$\mathrm{H},-2.7019387912,0.0347162069,1.300193577$

$\mathrm{H}, 1.5851756446,0.0612387944,1.3997152376$

$0,-0.5154056022,0.1322860261,2.8602625015$

$\mathrm{H},-1.4094631528,0.1404613845,3.2179238579$

$F, 2.3947275851,1.2516184293,-1.5632683111$

F,1.9560851954,-0.6746538396,-2.4642474919

$F, 2.9983266039,-0.5754784706,-0.5573579223$

$\mathrm{HF}=-644.707767 \quad \mathrm{NImag}=0$

\section{3- $\mathrm{CF}_{3}-\mathrm{C}_{6} \mathrm{H}_{4} \mathrm{O}^{-}$}

Brutoformula: C7H4F3O1(1-)

$-1,1$

$\mathrm{H},-0.5756345484,-0.1418020022,-2.1760370388$

C, $-0.5983003408,-0.080139149,-1.0959361073$

C, $-0.6475704295,0.0652703178,1.8069848203$

C, $0.5876914232,-0.0475388309,-0.3440629596$

C, $-1.8063691426,-0.0422578833,-0.3882265308$

C, $-1.843627313,0.0274358953,0.9951793984$

C, $0.5804467824,0.0248651938,1.0432528567$

C, $1.8945256721,-0.0091395298,-1.0689506017$

$\mathrm{H},-2.7418855559,-0.0719508293,-0.943878116$

$\mathrm{H},-2.7942533302,0.0540803997,1.5207607987$

$\mathrm{H}, 1.5106065493,0.0475615039,1.5995488485$

$0,-0.6665582328,0.1270373456,3.0698545004$

F,2.9364176761,-0.517811898,-0.3572566791

$F, 2.2843081137,1.2627164105,-1.4120956819$

F,1.8802026774,-0.698326944,-2.2491375089

$\mathrm{HF}=-644.1610414 \mathrm{NImag}=0$

\section{4- $\mathrm{CF}_{3}-\mathrm{C}_{6} \mathrm{H}_{4} \mathrm{OH}$}

Brutoformula: $\mathrm{C} 7 \mathrm{H} 5 \mathrm{~F} 3 \mathrm{O} 1$

0,1

C,-0.058730306,-0.0028131414,-2.4432422475
C, $-0.0467414701,0.0303418285,-0.9440342735$

C, $-0.0403505114,-0.0101349171,1.8432893721$

C,-1.2527190253,-0.0037948273,-0.2372446975

C, $1.1609132732,0.0404329894,-0.2482861163$

C, $1.1670438005,0.0227578375,1.1423870607$

C,-1.2533262142,-0.0223216747,1.1492969402

$\mathrm{H},-2.1936370332,-0.0082599423,-0.7736280059$

$\mathrm{H}, 2.0982230331,0.0703561197,-0.7891108251$

$\mathrm{H}, 2.109883305,0.0369739451,1.6802591157$

$\mathrm{H},-2.1797160429,-0.0437944638,1.7094556927$

O,-0.101172618,-0.0274083251,3.2048437986

$\mathrm{F},-0.1767199386,-1.2684353023,-2.9282312943$

$F, 1.0746389638,0.5058202105,-2.9795698629$

$\mathrm{F},-1.0947958617,0.697558286,-2.9631944841$

$\mathrm{H}, 0.7872066436,-0.0172786237,3.577009828$

$\mathrm{HF}=-644.7092441 \mathrm{NImag}=0$

\section{4- $\mathrm{CF}_{3}-\mathrm{C}_{6} \mathrm{H}_{4} \mathrm{O}$}

Brutoformula: C7H4F3O1(1-)

$-1,1$

C, $0 ., 0.0036110806,-2.1882999346$

C, $-0.0000000001,-0.0511076721,-0.7221859894$

C, $0.0000000001,0.0155173664,2.1728264825$

C, $1.2085634644,-0.0267332336,0.0048672372$

C, $-1.2085634646,-0.0267332336,0.0048672372$

C,-1.2164888864,0.0000439614,1.3827150615

C, $1.2164888866,0.0000439616,1.3827150615$

$\mathrm{H}, 2.1505286224,-0.0394915111,-0.5363141128$

$\mathrm{H},-2.1505286227,-0.0394915112,-0.5363141127$

$\mathrm{H},-2.1562227192,0.0067745163,1.9273026995$

$\mathrm{H}, 2.1562227194,0.0067745168,1.9273026993$

$0,-0.0000000001,0.0381875761,3.4326025444$

$F, 0.0000000006,1.2861353981,-2.7294310971$

F,-1.0941928352,-0.588311973,-2.7630244173

$F, 1.0941928349,-0.5883119738,-2.7630244172$

$\mathrm{HF}=-644.1673034 \mathrm{~N} I \mathrm{mag}=0$

\section{3,5- $\left(\mathrm{CF}_{3}\right)_{5}-\mathrm{C}_{6} \mathrm{H}_{3} \mathrm{OH}$}

Brutoformula: $\mathrm{C} 8 \mathrm{H} 4 \mathrm{~F} 6 \mathrm{O} 1$

0,1

$\mathrm{H},-0.3323034604,0.0031723373,-2.6998721466$

C, $-0.3101485001,0.0045211888,-1.6179386863$

C, $-0.2931687377,0.0325311433,1.1638115144$

C, $-1.5146244391,-0.0133993503,-0.9120389354$

C, $0.8903106177,0.0375168944,-0.9188390766$

C, $0.9153233646,0.0491192567,0.4761988068$

C,-1.5091784473,0.0001163099,0.4817258042

O,-2.6603543583,-0.0373869292,-1.6464920503

C,2.1903692822,0.0060222019,-1.6831466157

$\mathrm{H}, 1.8537492674,0.0864849971,1.0108586092$

$\mathrm{H},-2.4423160988,-0.0035064707,1.0343306246$

C, $-0.3008844954,-0.0040545247,2.6708202254$

$\mathrm{H},-3.4318847784,-0.045030141,-1.0696035486$

$F, 2.594543982,-1.2663386655,-1.9198272108$ 
$F, 3.1897488136,0.6133874867,-1.0092374439$ $F, 2.0892596956,0.6115999001,-2.8857009152$ F,-0.4069944415,-1.2734133562,3.1359876055 F,-1.3454007346,0.6837094203,3.1874742556 $F, 0.8239534693,0.5149483003,3.2014891827$

$H F=-981.8545103 \quad \mathrm{NImag}=0$

\section{3,5- $\left(\mathrm{CF}_{3}\right)_{5}-\mathrm{C}_{6} \mathrm{H}_{3} \mathrm{O}^{-}$}

Brutoformula: C8H3F6O1(1-)

$-1,1$

$\mathrm{H},-0.4942893895,0.0188009144,-2.7702722268$ C, $-0.4971214681,0.0210040902,-1.6864972787$ C, $-0.5007313552,0.0440754383,1.0922252427$ C, $-1.7856110744,0.0171692373,-1.0302748708$ C, $0.6946149822,0.0372570896,-0.9764178446$ $\mathrm{C}, 0.7323215505,0.0467810944,0.4243147733$

C,-1.7102505611,0.0275006471,0.4147248854 O,-2.8771399218,0.0078159936,-1.6592197907 C, $1.9905819484,-0.0261184315,-1.7254139621$ $\mathrm{H}, 1.6675058301,0.0749796949,0.9635059211$ $\mathrm{H},-2.6475634365,0.030019625,0.9581783859$ C, $-0.4937497276,-0.0086685463,2.5893083038$ $\mathrm{F}, 0.5222979525,0.7119528633,3.1452268001$ $F, 2.4029478116,-1.3100649441,-1.9661354814$ $F, 3.0246764701,0.5616766413,-1.0601313056$ $\mathrm{F}, 1.9433349088,0.5702933521,-2.945718128$ F,- $0.3356845962,-1.2799230667,3.0748707578$ $\mathrm{F},-1.6361399748,0.455448276,3.1577257836$

$\mathrm{HF}=-981.3241116 \mathrm{NImag}=0$

\section{$\mathrm{C}_{6}\left(\mathrm{CF}_{3}\right)_{5} \mathrm{CH}(\mathrm{CN})_{2}$}

Brutoformula: C14H1F15N2

0,1

C,-0.0709306763,-0.0054771055,-3.2154852216 C, $-0.0190366142,-0.1791642262,-1.6724038101$ C, $-0.0574818873,0.1844998552,1.1287397969$ C, $1.1893534521,-0.0189057431,-0.9668360834$ C, $-1.2276291063,-0.3143368678,-0.9621008926$ C, $-1.2553717681,-0.0263389793,0.4125183282$ C, $1.1680963108,0.2026130908,0.4299047867$ C, $2.5312201029,-0.2414517648,-1.7187638184$ C,-2.4502971893,-0.9854554714,-1.6433641536 C,-2.5998295153,0.1704884937,1.1439159005 C,2.4798510742,0.5641214362,1.1689664991 C, $-0.155194842,0.2489156806,2.6871816778$ $\mathrm{H},-1.1332421447,-0.1436442318,2.9655747094$ C,-0.1096379515,1.6033565995,3.2601425299 C, $0.7603873637,-0.6781237734,3.3711946127$ $\mathrm{N},-0.1390017067,2.6548440982,3.7263376696$ $\mathrm{N}, 1.3901142087,-1.4563444862,3.9389008686$ F,3.0921598177,0.8849163206,-2.167648337 $F, 2.3454126045,-1.0621292929,-2.7667378047$ F,3.4132787594,-0.8712128203,-0.9260896076 $F, 3.278575012,1.3065204128,0.3884491949$
$F, 3.1565535174,-0.5100909824,1.5854229694$ $F, 2.2286289792,1.3245458978,2.2518976006$ $\mathrm{F}, 0.863096304,0.8843466661,-3.5966918737$ F,-1.2555623972,0.5391272774,-3.5569146105 F,0.0903328979,-1.1175536678,-3.932229175 F,-3.3600447452,-0.1492369975,-2.1442888369 F,-3.0584205517,-1.7937667776,-0.749309757 $\mathrm{F},-2.0352723762,-1.7914089732,-2.6345928409$ F,-2.5224040201,1.2506559696,1.9543041077 F,-2.9205976275,-0.8881566087,1.9188271384 $F,-3.617105286,0.4138469742,0.3211784317$

$H F=-2141.7705722 \quad N I m a g=0$

$\mathrm{C}_{6}\left(\mathrm{CF}_{3}\right)_{5} \mathrm{C}(\mathrm{CN})_{2}$

Brutoformula: C14F15N2(1-)

$-1,1$

C,-0.1004290976,-0.6974866442,-3.0287867157 C, $-0.0498465013,-0.3799308989,-1.5394456288$ C, $0.0526420951,0.0689145021,1.2939866974$ C, $1.1840265101,-0.0905620076,-0.8919833839$ C,-1.2437267032,-0.189603082,-0.7887588062 C, $-1.194013581,0.1863171284,0.5593733293$ C, $1.2191025815,0.2843186208,0.4568924181$ C, $2.5287700794,-0.3220355248,-1.6079225356$ C,-2.6213430591,-0.53234025,-1.3881739609 C, $-2.3504804802,0.9402717131,1.2160107909$ C,2.3624785546,1.1309494522,1.0162995287 C, $0.1222065844,-0.2407876014,2.6581801394$ $\mathrm{F},-3.0233604065,1.6779184007,0.290698933$ C,- $-1.0025770216,-0.55786827,3.4684178825$ C, $1.332109357,-0.4550521472,3.3739601715$ $\mathrm{N},-1.8513523934,-0.8672544964,4.194251918$ $\mathrm{N}, 2.2601596782,-0.6849433788,4.028812677$ $F, 2.9242247078,0.6775562011,-2.4196462772$ $F, 2.4858332324,-1.460635001,-2.3405670019$ $F, 3.5330534104,-0.521613263,-0.7311142973$ $\mathrm{F}, 2.894661484,1.9164540811,0.0402390775$ $F, 3.3914149437,0.4825705953,1.5871969455$ $F, 1.883610332,1.9911937615,1.9469573592$ $F, 0.9132093721,-0.1263360689,-3.7184933354$ $\mathrm{F},-1.2110459867,-0.2105126306,-3.6276828288$ F,-0.059714916,-2.024517408,-3.3086707384 F,-3.1649484892,0.4296420091,-2.1584377722 F,-3.5272133824, $-0.8111277479,-0.429607526$

$\mathrm{F},-2.5484001609,-1.6654650223,-2.1269743322$ F,-1.8658860446,1.8385287871,2.1070033773 F,-3.2731646969,0.2134361924,1.8679838944

$H F=-2141.2977378 \quad N I m a g=0$

\section{2,3,4,6- $\mathrm{C}_{6}\left(\mathrm{CF}_{3}\right)_{4} \mathrm{H}-\mathrm{CH}(\mathrm{CN})_{2}$}

Brutoformula: C13H1F12N2(1-)

$-1,1$

C,0.5181259306,0.378339421,-3.194864969

C, $0.1669161847,0.2073840785,-1.7394169762$ 
C, $-0.1458417716,0.3036343031,1.1139529285$ C, $0.2573614949,-0.9845973323,-0.9851011362$ C, $-0.335838259,1.3393392126,-1.0900795449$ C, $-0.5358706293,1.4065193371,0.2711517661$ C, $-0.0418659035,-0.9610922832,0.3874033196$ C, $0.6688913977,-2.2799675321,-1.6886325821$ $\mathrm{H},-0.5598615271,2.2129115855,-1.6807458691$ C,-1.107464463,2.7005962448,0.7911096172 C, $-0.5319197908,-2.230503173,1.083110913$ C, $0.1526203591,0.3780513469,2.4942198984$ $\mathrm{N},-0.3588687147,2.2611695014,4.1764379862$ C, $0.9292503344,-0.6142477661,3.1506313358$ C, $-0.1513360895,1.4476183302,3.3758889098$ $\mathrm{F}, 0.4049354726,-3.0424623077,1.6053339734$ F,-1.2523807229,-2.9925595711,0.2078904984 $\mathrm{F},-1.3950175205,-1.9418013894,2.0833880513$ F,- $0.1852566733,3.490430221,1.3914690505$ $F,-2.1132219673,2.5034213261,1.670291652$ F,-1.6364476534,3.4588037977,-0.2113755284 $F, 1.1267968106,-3.2241748262,-0.8494450716$ $F, 1.6880769221,-2.0490755883,-2.5517918719$ F,- $0.3338879798,-2.8346789683,-2.4054785368$ $F, 1.8572205383,0.4643136894,-3.4213829192$ F,- $0.0022821868,1.5336381195,-3.6946120437$ F,0.0520261212,-0.6026370648,-4.0039165611 $\mathrm{N}, 1.6048885133,-1.3529025184,3.7371211802$

$H F=-1804.1897772 \quad N I m a g=0$

\section{2,3,4,6- $\mathrm{C}_{6}\left(\mathrm{CF}_{3}\right)_{4} \mathrm{H}-\mathrm{C}(\mathrm{CN})_{2}$}

Brutoformula: C13H1F12N2(1-)

$-1,1$

C, $0.5181259306,0.378339421,-3.194864969$

C, $0.1669161847,0.2073840785,-1.7394169762$ C, $-0.1458417716,0.3036343031,1.1139529285$ C, $0.2573614949,-0.9845973323,-0.9851011362$ C, $-0.335838259,1.3393392126,-1.0900795449$ C, $-0.5358706293,1.4065193371,0.2711517661$ C, $-0.0418659035,-0.9610922832,0.3874033196$ C, $0.6688913977,-2.2799675321,-1.6886325821$ $\mathrm{H},-0.5598615271,2.2129115855,-1.6807458691$ C, $-1.107464463,2.7005962448,0.7911096172$ C,- $-0.5319197908,-2.230503173,1.083110913$ C, $0.1526203591,0.3780513469,2.4942198984$ $\mathrm{N},-0.3588687147,2.2611695014,4.1764379862$ C, $0.9292503344,-0.6142477661,3.1506313358$ C, $-0.1513360895,1.4476183302,3.3758889098$ $\mathrm{F}, 0.4049354726,-3.0424623077,1.6053339734$ $F,-1.2523807229,-2.9925595711,0.2078904984$ $\mathrm{F},-1.3950175205,-1.9418013894,2.0833880513$ $F,-0.1852566733,3.490430221,1.3914690505$ $F,-2.1132219673,2.5034213261,1.670291652$ F,-1.6364476534,3.4588037977,-0.2113755284 F,1.1267968106,-3.2241748262,-0.8494450716 $F, 1.6880769221,-2.0490755883,-2.5517918719$ $\mathrm{F},-0.3338879798,-2.8346789683,-2.4054785368$
$F, 1.8572205383,0.4643136894,-3.4213829192$ F,-0.0022821868,1.5336381195,-3.6946120437 F,0.0520261212,-0.6026370648,-4.0039165611 $\mathrm{N}, 1.6048885133,-1.3529025184,3.7371211802$

$H F=-1804.1897772 \quad \mathrm{NImag}=0$

\section{2,4,6- $\left(\mathrm{CF}_{3}\right)_{3}-\mathrm{C}_{6} \mathrm{H}_{2} \mathrm{OH}$}

Brutoformula: $\mathrm{C} 9 \mathrm{H} 3 \mathrm{~F} 9 \mathrm{O} 1$

0,1

$0,-0.0762775084,-0.0777061089,-2.6049092766$ C, $0.0058016047,-0.020478476,-1.2638555784$ $\mathrm{C}, 0.0027142365,0.0937205849,1.5395683698$ C, $1.2159452482,0.0204571113,-0.5454616955$ C, $-1.2078452154,-0.0106094166,-0.5479833361$ C, $-1.2014729076,0.0435249082,0.8375895346$ C, $1.2046451529,0.0777051566,0.8465952948$ C, $2.535827937,-0.0687710822,-1.2618795234$ C,-2.520236186,-0.0417995103,-1.2993682314 $\mathrm{H},-2.1423869009,0.0593577248,1.371051187$ $\mathrm{H}, 2.1422013747,0.1155777338,1.3841078616$ C,- $0.0141411097,0.1144012707,3.0437844662$ $\mathrm{H}, 0.7744312795,0.0938065724,-3.0275762007$ $F, 3.568458622,0.3110510317,-0.498313045$ $F, 2.5558387853,0.7219633304,-2.3811044116$ $F, 2.7932019526,-1.3243145824,-1.6958616765$ F,-2.6618131268,1.0248145117,-2.1130721195 F,-3.5709528691,-0.0285558443,-0.4486478232 F,-2.643974589,-1.1493187845,-2.0580228069 $\mathrm{F},-0.9839892646,0.9237232757,3.5238080827$ $F, 1.1592708055,0.538307672,3.5580589196$ F,-0.2484245658,-1.1172257701,3.559572165

$H F=-1318.9964267 \quad N I m a g=0$

\section{2,4,6- $\left(\mathrm{CF}_{3}\right)_{3}-\mathrm{C}_{6} \mathrm{H}_{2} \mathrm{O}^{-}$}

Brutoformula: $\mathrm{C} 9 \mathrm{H} 2 \mathrm{~F} 9 \mathrm{O} 1(1-)$

$-1,1$ $0,0.0082190492,-0.112293484,-2.6173965901$ C, $0.0046574494,-0.0661559933,-1.3763649294$ C, $-0.0030755923,0.0293616804,1.5188925488$ C, $1.2252579801,0.020024217,-0.5717135965$ C,-1.2203982047,-0.0958513585,-0.5744232916 C, $-1.2070731479,-0.0459219139,0.8038122387$ C, $1.2042221938,0.0683249517,0.8064839322$ C, $2.5320848048,0.0497401464,-1.2944074618$ C,-2.5225780402,-0.1897518004,-1.3000121142 $\mathrm{H},-2.1442082751,-0.0735367378,1.3455838221$ $\mathrm{H}, 2.1385670887,0.1293784342,1.3503330649$ C,- $0.0106160088,0.1541390562,2.9914452854$ F,-1.0754280574,-0.4644246525,3.5775357999 $F, 3.5990011473,0.1304302733,-0.4350205702$ $F, 2.6676845951,1.1114686766,-2.1313067719$ $F, 2.7642650018,-1.0575529789,-2.047249278$ $F,-2.7561008015,0.8544808576,-2.1373247536$ $\mathrm{F},-3.5942436578,-0.2103812707,-0.4429959844$ 
$\mathrm{F},-2.6473617624,-1.3139646692,-2.0532409117$ F,-0.0728010454,1.4552672141,3.4475466261 $F, 1.106651268,-0.3609843101,3.5799425288$

$H F=-1318.4899137 \quad \mathrm{NImag}=0$

\section{1,2-( $\left(\mathrm{CF}_{3}\right)_{2}-\mathrm{C}_{6} \mathrm{H}_{4}$}

Brutoformula: $\mathrm{C} 8 \mathrm{H} 4 \mathrm{~F} 6$

0,1

C,- $0.0143754535,-0.6868738229,-2.5129627509$

C, $-0.009713138,-0.7891646559,-0.9974163296$

C, $-0.0702242642,-0.7872173689,1.8036791336$ C, $0.0285038795,-2.0092106687,-0.2915183658$

C, $-0.0895967524,0.4068431251,-0.2831210133$

C, $-0.1194210092,0.4132916484,1.1084468736$

C, $0.0040502764,-1.9879247576,1.1034623837$

C, $0.1409672963,-3.3700836367,-0.956730558$

$\mathrm{H},-0.122314582,1.3419419728,-0.8248617699$

$\mathrm{H},-0.1798808075,1.355796528,1.6393255482$

$\mathrm{H}, 0.0372005718,-2.9232101731,1.6448860127$

$\mathrm{H},-0.0904286753,-0.7982304707,2.8868481165$

$F, 0.8778353592,-1.5080010051,-3.0966380623$

$F, 0.2927631073,0.5670231363,-2.9204592602$

$\mathrm{F},-1.2303646551,-0.9686865619,-3.0312782042$

$F, 1.4049740451,-3.6285938622,-1.3597950858$

F,-0.6626233709,-3.4974822858,-2.0284543204

F,-0.1973513377,-4.3631820679,-0.1012340028

$\mathrm{HF}=-906.597689 \quad \mathrm{NImag}=0$

1,2- $\left(\mathrm{CF}_{3}\right)_{2}-\mathrm{C}_{6} \mathrm{H}_{3}{ }^{-}$

Brutoformula: C8H3F6(1-)

$-1,1$

C, $0.0107625497,-0.6895151591,-2.4951506383$ C, $-0.0279219739,-0.8012044799,-0.9908974314$ C, $0.0148171926,-0.7860774252,1.7881918964$

C, $0.0527472777,-2.0083150851,-0.2378765182$ C, $-0.1610213879,0.4052425543,-0.2987252163$ C, $-0.1623273738,0.4090036137,1.0954980758$ C, $0.1154279386,-2.0520551853,1.1685896902$ C, $0.1114657819,-3.3591678022,-0.9254122059$ $\mathrm{H},-0.2415001979,1.3345842741,-0.8469763202$ $\mathrm{H},-0.274689036,1.3559259662,1.6231376017$ $\mathrm{F},-0.3402806398,-4.3737231087,-0.1650931011$ $\mathrm{H}, 0.077108997,-0.7253729412,2.8779798067$ F,0.9276605586,-1.4920534519,-3.0865425976 $F, 0.3272030317,0.5776710459,-2.9169371204$ $F,-1.1845856822,-0.9586018384,-3.097466179$ $F, 1.3844920694,-3.713680314,-1.2967429192$ F,-0.6293585999,-3.4237983282,-2.0787320502

$\mathrm{HF}=-905.9852834 \mathrm{NImag}=0$

$1,4-\left(\mathrm{CF}_{3}\right)_{2}-\mathrm{C}_{6} \mathrm{H}_{4}$

Brutoformula: C8H4F6

0,1
C,0.,-0.0064307879,-2.8947783048

C, $0 ., 0.0432845153,-1.3884018008$

C, $0 ., 0.0432845153,1.3884018008$

C, $-1.2102625336,0.0426811637,-0.6953074605$

C, $1.2102625336,0.0426811637,-0.6953074605$

$\mathrm{C}, 1.2102625336,0.0426811637,0.6953074605$

C,-1.2102625336,0.0426811637,0.6953074605

$\mathrm{H},-2.1470747792,0.0525585524,-1.2373193924$

$\mathrm{H}, 2.1470747792,0.0525585524,-1.2373193924$

$\mathrm{H}, 2.1470747792,0.0525585524,1.2373193924$

$\mathrm{H},-2.1470747792,0.0525585524,1.2373193924$

C, $0 .,-0.0064307879,2.8947783048$

F,0.,-1.2832939153,-3.3500188656

$F, 1.0879031746,0.5950684336,-3.4234838222$

F,-1.0879031746,0.5950684336,-3.4234838222

F,0.,-1.2832939153,3.3500188656

$\mathrm{F},-1.0879031746,0.5950684336,3.4234838222$

$F, 1.0879031746,0.5950684336,3.4234838222$

$\mathrm{HF}=-906.6077895 \mathrm{NImag}=0$

2,5- $\left(\mathrm{CF}_{3}\right)_{2}-\mathrm{C}_{6} \mathrm{H}_{3}^{-}$

Brutoformula: C8H3F6(1-)

$-1,1$

C, $-0.0150837001,0.0086678839,-2.8632788263$

C, $-0.0161787218,0.0491586334,-1.3694610548$

C, $-0.0465337733,0.0024649244,1.3700905044$

C,-1.218063059,-0.0898179074,-0.6597790249

C, $1.1997369771,0.1689874251,-0.6867974096$

C, $1.1798375106,0.1493138416,0.7026476142$

C,-1.3085584951,-0.1219548796,0.7514741557

$\mathrm{H},-2.1315582999,-0.1707654704,-1.249568973$

$\mathrm{H}, 2.1317145385,0.283098031,-1.2267991268$

$\mathrm{H}, 2.111941421,0.2484084373,1.2498824786$

$\mathrm{F}, 1.2382797253,0.0916530166,3.4122207264$

C, $-0.0279398134,-0.0227120058,2.8789891935$

F,-0.0364989422,-1.2670372007,-3.3665363909

$F, 1.0861198956,0.5920615764,-3.4251045902$

$\mathrm{F},-1.0885255599,0.6297573269,-3.4237579782$

$F,-0.5273921369,-1.1710840486,3.40880089$

F, $-0.7381384496,0.9884950526,3.4480634216$

$H F=-905.998971 \quad$ NImag $=0$

1,2,3-( $\left(\mathrm{CF}_{3}\right)_{3}-\mathrm{C}_{6} \mathrm{H}_{3}$

Brutoformula: $\mathrm{C} 9 \mathrm{H} 3 \mathrm{~F} 9$

0,1

C, $0.1482771569,2.6002339618,-0.0865124477$

C, $0.7765932327,1.2121458218,0.0329471903$

C,2.1710658884,-1.1962201956,0.0973523159

C, $0.0511063406,0 ., 0.0596029132$

C, $2.1710658884,1.1962201956,0.0973523159$

C,2.8669084479,0.,0.15634929

C, $0.7765932327,-1.2121458218,0.0329471903$

C, $-1.4796136174,0 ., 0.1255110567$

$\mathrm{H}, 2.7100612441,2.1327432492,0.0878338421$ 
$\mathrm{H}, 3.9480596197,0 ., 0.2173917431$

C, $0.1482771569,-2.6002339618,-0.0865124477$ $\mathrm{H}, 2.7100612441,-2.1327432492,0.0878338421$ F, $-0.8724544726,2.6354961931,-0.9600016431$ $F, 1.0618795708,3.4931079652,-0.5395866987$ $F,-0.2856803285,3.0771948634,1.0966576843$ $\mathrm{F},-2.0358557783,0 .,-1.1010314302$

F,-1.9496960178,1.0714882116,0.7885972788 F,-1.9496960178,-1.0714882116,0.7885972788 F,- $0.8724544726,-2.6354961931,-0.9600016431$ $F,-0.2856803285,-3.0771948634,1.0966576843$ F,1.0618795708,-3.4931079652,-0.5395866987

$H F=-1243.726878 \quad N I m a g=0$

2,3,4-( $\left(\mathrm{CF}_{3}\right)_{3}-\mathrm{C}_{6} \mathrm{H}_{2}$

Brutoformula: $\mathrm{C} 9 \mathrm{H} 2 \mathrm{F9}$ (1-)

$-1,1$

C, $0.1755321573,2.5451844772,-0.2061502785$ C, $0.7931175828,1.1863521691,-0.0061599513$ C, $2.2122466249,-1.2808974553,0.3442582636$ C, $0.0699028043,-0.0283975976,0.0947791447$ C,2.1878613008,1.1608704528,0.1057659989 C, $2.843248426,-0.0306012658,0.406007131$ C, $0.8288732518,-1.2311422647,0.105268263$ C, $-1.4340454315,-0.014352309,0.2873639222$ $\mathrm{H}, 2.7389718777,2.0896652608,0.0019689817$ $\mathrm{H}, 3.8999155023,0.0316789969,0.6725589766$ C, $0.1994673922,-2.5800259107,-0.2028723363$ $F, 1.0447129448,-3.3940704367,-0.8709952189$ $\mathrm{F},-0.9379704586,2.5444280147,-0.9773712829$ $F, 1.0411352913,3.3990801612,-0.8302643068$ $\mathrm{F},-0.1603649801,3.1807866524,0.9545443755$ F,-2.1478456975,0.0112486753,-0.8687501723 F,-1.8578710574,1.0645022877,1.0074615365 F,-1.871783983,-1.086730811,0.9867409993 F,-0.9105082362,-2.4951928449,-0.9993864843 F,- $0.1879607156,-3.2777501469,0.9042328981$

$\mathrm{HF}=-1243.132011 \mathrm{NImag}=0$

\section{1,2,5-( $\left(\mathrm{CF}_{3}\right)_{3}-\mathrm{C}_{6} \mathrm{H}_{3}$}

Brutoformula: $\mathrm{C} 9 \mathrm{H} 3 \mathrm{~F} 9$

0,1

C,- $0.762141159,0.1043922056,-2.557872765$

C, $-0.6603476425,0.0187130171,-1.0420404464$

C, $-0.6674783939,-0.0805794471,1.7518365837$ C, $0.5590348132,-0.0027982553,-0.3356800315$ C, $-1.8588333478,0.0009080325,-0.3294006936$ C,- $-1.8712134978,-0.0476035443,1.0601201349$ C, $0.5386093126,-0.0569713604,1.0563073558$ C, $1.9272303741,-0.0134561199,-1.0011234025$ $\mathrm{H},-2.7948989694,0.0180700065,-0.8695712828$ $\mathrm{H},-2.8125707877,-0.0677090359,1.5937072321$ $\mathrm{H}, 1.4722058811,-0.0822210063,1.5999782997$ C, $-0.6557195949,-0.0836465313,3.2608991179$
$\mathrm{F},-0.5406144198,1.3607993473,-2.997662784$ $F,-1.9987897323,-0.2411984376,-2.9821116394$ $F, 0.103672273,-0.710512165,-3.1840516498$ $F, 2.0181471947,0.8707480439,-2.0096879709$ $\mathrm{F}, 2.2332051118,-1.2330064213,-1.4911107893$ $F, 2.8996805522,0.3027033775,-0.1170094443$ F, $-0.5851457822,1.1757734894,3.7536790049$ $\mathrm{F}, 0.4013502537,-0.7592069003,3.7562614778$ F,-1.7714589297,-0.6440878833,3.7714283096

$H F=-1243.7422923 \quad N I m a g=0$

\section{2,3,6- $\left(\mathrm{CF}_{3}\right)_{3}-\mathrm{C}_{6} \mathrm{H}_{2}^{-}$}

Brutoformula: $\mathrm{C} 9 \mathrm{H} 2 \mathrm{F9}$ (1-)

$-1,1$

C, $0.7459350846,0.0007683515,-2.5262196788$ C, $0.631069157,-0.0495957064,-1.0195197134$ C, $0.6319872054,0.0274617385,1.7450724971$ C,- $0.5792945238,-0.066277748,-0.2773544922$ C, $1.8413977581,-0.0866063759,-0.3215615701$ C, $1.847996963,-0.0758458988,1.0694294407$ C, $-0.6294633164,0.0177603045,1.12481634$ C, $-1.9309922098,-0.1477845518,-0.9653224942$ $\mathrm{H}, 2.7764638671,-0.1029025725,-0.8645175611$ $\mathrm{H}, 2.795312296,-0.1200517401,1.5970617896$ $F, 1.9255153444,0.2141036227,3.7669020032$ C, $0.6520299388,0.1614862853,3.2489501087$ F,0.5993961074,-1.2156729554,-3.1208658664 $F, 1.9771110382,0.4415795129,-2.9351891727$ $\mathrm{F},-0.141645172,0.8334433285,-3.114632081$ $F,-1.9159579633,-0.9008520396,-2.1094120405$ F,-2.4063929485,1.0793081078,-1.3425712106 $F,-2.8879928156,-0.6940120642,-0.1952326911$ F, $0.0514502584,-0.8744219198,3.8908391709$ $\mathrm{F}, 0.0389858727,1.2870528427,3.6932411265$

$H F=-1243.1522118 \quad \mathrm{NImag}=0$

\section{1,3,5-( $\left(\mathrm{CF}_{3}\right)_{3}-\mathrm{C}_{6} \mathrm{H}_{3}$}

Brutoformula: $\mathrm{C} 9 \mathrm{H} 3 \mathrm{~F} 9$

0,1

C, $-0.0000002993,-0.0050021952,-2.8972561979$

C, $-0.0000000603,0.0357787999,-1.388497451$ C, $0.0000001356,0.0287756311,1.3970777043$ C,-1.2095888237,0.0326955457,-0.698450487 C, $1.2095887785,0.0326956981,-0.6984506745$ C, $1.2023489158,0.0320804931,0.6941582647$ C, $-1.2023487668,0.0320803213,0.6941584262$ $\mathrm{H},-2.1467552469,0.0488867034,-1.2388860191$ $\mathrm{H}, 2.1467551134,0.0488869714,-1.2388863448$ C, $2.5090289593,-0.01303996,1.4482898507$ C, $-2.509028669,-0.0130403386,1.4482902451$ $\mathrm{H}, 0.0000002075,0.0413085565,2.4791440829$ F, $-0.0000023748,-1.2796758725,-3.3545698916$ $F, 1.0880791381,0.5991284319,-3.417030748$ $F,-1.0880780529,0.5991318054,-3.4170304149$ 
F,-2.8895340206,-1.2888085303,1.6962149235

F,-3.5100375125,0.5684071418,0.7562792069

$\mathrm{F},-2.4235891841,0.6100554216,2.6414764268$

$F, 2.8895348351,-1.2888081001,1.6962139944$

$F, 2.4235894602,0.6100553404,2.6414762653$

$F, 3.5100374675,0.568408141,0.7562788383$

$H F=-1243.7519654 \quad N I m a g=0$

\section{2,4,6- $\left(\mathrm{CF}_{3}\right)_{3}-\mathrm{C}_{6} \mathrm{H}_{2}^{-}$}

Brutoformula: C9H2F9(1-)

$-1,1$

C,2.4852283012,0.1855985034,1.4514694828

C, $1.1704685634,0.0943677559,0.7147218499$

C, $-1.2067590897,-0.0629113245,-0.6718260708$

C, $1.2053605358,-0.0030858691,-0.6774113985$

C, $0.0004541178,0.1220973092,1.5027812206$

C,-1.1703438434,0.0362459175,0.7201857081

C, $-0.0012633732,-0.078004944,-1.3772466299$

$\mathrm{H}, 2.1425974129,-0.0138093664,-1.2214209195$

$\mathrm{F},-2.6182063175,-0.955278476,2.3513902791$

C,-2.4847225058,0.0621549763,1.462779233

C, $-0.0005988211,-0.2413812294,-2.8580122273$

$\mathrm{H},-2.14472275,-0.1200244959,-1.2115558893$

$F, 3.5767262475,0.1416992442,0.6163320918$

$\mathrm{F}, 2.6738825574,-0.8252505649,2.3376673728$

$\mathrm{F}, 2.6243696385,1.3368908366,2.1584649595$

F, $-1.1072376915,0.2801379536,-3.4540428043$

$F, 0.0375100288,-1.5531068808,-3.2586047281$

$F, 1.0697158638,0.3449791384,-3.4603241651$

F,-3.5762906157,-0.0382204648,0.6325913653

$F,-2.6787828193,1.2062989126,2.1685622737$

$H F=-1243.1658852 \quad N I m a g=0$

\section{1,2,4,5- $\left(\mathrm{CF}_{3}\right)_{4}-\mathrm{C}_{2} \mathrm{H}_{2}$}

Brutoformula: $\mathrm{C} 10 \mathrm{H} 2 \mathrm{~F} 12$

0,1

$\mathrm{H},-0.0006430024,-0.0170952027,2.4533611303$

C, $-0.0003613356,-0.0095730586,1.3740754131$

C, $0.0003478525,0.0095873798,-1.3740820603$

C, $-1.2189540122,0.0354087795,0.7013343848$

C, $1.2185693017,-0.0451784991,0.7014103125$

C, $1.2184606316,-0.0477281267,-0.7014324248$

C, $-1.2181236009,0.0575184566,-0.7013253604$

C,-2.476469677,0.1004721939,1.5588796139

C, $2.4756791106,-0.1222386324,1.5585568069$

C,2.4777620034,-0.0665992655,-1.5586138192

C,-2.4769791105,0.0883674846,-1.5588212996

$\mathrm{H}, 0.0006236873,0.0171141755,-2.4533673154$

$F,-3.4522979941,-0.6988425866,1.0984225821$

$\mathrm{F},-2.9612561703,1.3563541311,1.6245227601$

$F,-2.2170538098,-0.285234801,2.8265920488$

F,-3.3889333496,0.9599346223,-1.0985554856

$\mathrm{F},-3.056195288,-1.12688244,-1.6241186641$

$F,-2.188969203,0.4528181101,-2.8266523287$
$F, 2.2156107516,0.2453295162,2.8315132145$ $\mathrm{F}, 3.4515733837,0.6837225749,1.109990834$ $F, 2.9606712094,-1.3788440526,1.6065369994$ $\mathrm{F}, 3.0569604506,1.149458664,-1.6066825591$ $\mathrm{F}, 2.190424994,-0.4133824627,-2.8315365193$ $F, 3.3895130626,-0.9444578585,-1.1100199061$

$H F=-1580.8752083 \quad$ NImag $=0$

\section{1,2,4,5-( $\left(\mathrm{CF}_{3}\right)_{4}-\mathrm{C}_{2} \mathrm{H}^{-}$}

Brutoformula: C10H1F12(1-)

$-1,1$

$F, 2.1142938272,1.1378219359,-2.5287897871$

C, $-0.0000000124,-0.0000000162,1.5477932817$

C, $-0.0000000146,-0.0000000031,-1.2547336593$

C, $1.1897845525,-0.0756228743,0.8333314416$

C, $-1.1897845797,0.0756228539,0.8333314452$

C, $-1.2106447022,-0.1177608889,-0.5720878793$

C, $1.2106446736,0.1177608713,-0.5720878786$

C, $2.4323170039,-0.4187695004,1.6262390915$

C,-2.4323170005,0.4187695602,1.6262391117

C,-2.4269413715,-0.4176855523,-1.407891078

C,2.426941366,0.4176854938,-1.4078910572

$\mathrm{H},-0.0000000133,-0.0000000093,-2.3362144117$

$F,-3.3584890707,-1.142824932,-0.7518080885$

$\mathrm{F},-2.1142937591,-1.1378218947,-2.5287898494$

F,-3.0548018921,0.6979130592,-1.8752724278

F,3.0548018124,-0.6979131318,-1.8752724594

$F, 3.3584891153,1.1428247459,-0.7518079965$

F,-3.0596675675,-0.6764078253,2.1433340086

F,-3.374625359,1.0696752935,0.8825719374

F,-2.1688104283,1.2279559896,2.6719501636

$F, 2.1688105395,-1.2279560877,2.6719500492$

$F, 3.059667419,0.6764079147,2.143334109$

$F, 3.3746254613,-1.069675002,0.8825718383$

$H F=-1580.3003285 \quad N I m a g=0$

\section{2,4,6-( $\left(\mathrm{NO}_{2}\right)_{3}-\mathrm{C}_{6} \mathrm{H}_{3}$}

Brutoformula: $\mathrm{C} 6 \mathrm{H} 3 \mathrm{~N} 3 \mathrm{O} 6$

0,1

$\mathrm{N}, 0.0000001312,0.0000012857,2.8576630127$

C, $0.0000000533,0.00000171,1.3694849221$

C, $-0.0000000558,0.0000025027,-1.406610361$

C,-1.2179989545,0.0000020158,0.7031436205

C, $1.2179990105,0.000001697,0.7031435236$

C, $1.1860697304,0.0000022182,-0.6848459953$

C,-1.1860697832,0.000002541,-0.6848459032

$\mathrm{H},-2.1539197225,0.0000019847,1.2436604325$

$\mathrm{H}, 2.1539198246,0.0000014168,1.2436602559$

$\mathrm{N}, 2.4750375015,0.000002344,-1.4287798624$

$\mathrm{N},-2.4750376326,0.0000030185,-1.4287796348$

$\mathrm{H},-0.0000001017,0.000002844,-2.4874020689$

O,-3.4994916863,-0.0000053279,-0.7655772496

$0,-2.4131982101,-0.0000048643,-2.6475759923$

O,-1.0864937954,-0.0000031424,3.4134076052 
$0,1.0864941166,-0.0000034656,3.4134074938$

$0,2.4131979515,-0.0000040071,-2.6475762152$

$0,3.4994916226,-0.0000047711,-0.7655775847$

$\mathrm{HF}=-845.9832814 \mathrm{NImag}=0$

\section{2,4,6- $\left(\mathrm{NO}_{2}\right)_{3}-\mathrm{C}_{6} \mathrm{H}_{2}^{-}$}

Brutoformula: $\mathrm{C} 6 \mathrm{H} 2 \mathrm{~N} 3 \mathrm{O} 6(1-)$

$-1,1$

$\mathrm{N}, 2.4962769511,-0.0129758076,1.5436556296$

C, $1.1463006035,0.0028187642,0.8569897341$

C,-1.2146900945,-0.0229552036,-0.5310156026

C, $1.21463021,0.0261184498,-0.5310368266$

C, $0.0001277158,-0.0052898027,1.6493406477$

C,-1.1461616417,-0.0084192399,0.857116891

C, $-0.0000784674,0.0037468961,-1.216434486$

$\mathrm{H}, 2.1504301473,0.0603833456,-1.0697712199$

O,-2.5739651675,0.4503641989,2.6740589642

$\mathrm{N},-2.4960264906,0.0030977191,1.5440558919$

$\mathrm{N},-0.0001850368,0.0084261355,-2.6783433012$

$\mathrm{H},-2.1505643738,-0.0537839386,-1.0698299268$

$0,1.0837111213,0.0063715958,-3.26171725$

$0,-1.0841708459,0.0142513161,-3.2615287311$

$0,3.462171005,0.4272998659,0.912084813$

$0,2.5743352789,-0.4669850298,2.6709567189$

O,-3.4619592435,-0.4334945016,0.9099990588

$\mathrm{HF}=-845.4234547 \quad \mathrm{NImag}=0$

\section{2,3,4,5,6-( $\left(\mathrm{NO}_{2}\right)_{5}-\mathrm{C}_{6} \mathrm{H}$}

Brutoformula: $\mathrm{C} 6 \mathrm{H} 1 \mathrm{~N} 5 \mathrm{O} 10$

0,1

$\mathrm{N}, 2.4421778819,0.0641444005,1.8170186626$

C, $1.1870036456,0.0625411039,1.0173132467$

C, $-1.203082715,-0.082376646,-0.3872846191$

C, $1.2069080795,0.0901797444,-0.3737651142$

C, $-0.0083347838,-0.0175436043,1.7120255984$

C, $-1.1968168029,-0.0831905397,1.0041781756$

C, $0.0052907576,0.0108060649,-1.0705611138$

$\mathrm{N}, 2.4889491238,0.2170415571,-1.1286529217$

$\mathrm{H},-0.0135416021,-0.02854548,2.7939909345$

$\mathrm{N},-2.4598023454,-0.1009305208,1.7912554403$

$\mathrm{N}, 0.0124217881,0.0256262831,-2.5632535006$

$\mathrm{N},-2.4776822384,-0.1931972625,-1.1571538147$

$0,2.3876624789,0.6071276591,2.9050977753$

$0,3.4003253525,-0.5058066461,1.3294049481$

$0,2.7730754235,-0.7150637499,-1.8517398497$

$0,3.1057396554,1.2451432021,-0.950139501$

$0,0.6093552979,0.9455909408,-3.084507185$

O,-0.5795402496,-0.8838839376,-3.108112767

$0,-2.7542203045,0.7535718732,-1.8639807462$

$0,-3.096748088,-1.2242200275,-1.0055301344$

$0,-3.4127208096,0.4801865825,1.3065111233$

O,-2.4164195453,-0.6672009972,2.8678853596

$H F=-1255.0309872 \quad \mathrm{NImag}=0$

\section{2,3,4,5,6-( $\left(\mathrm{NO}_{2}\right)_{5}-\mathrm{C}_{6}^{-}$}

Brutoformula: C6N5O10(1-)

$-1,1$

$\mathrm{N}, 2.423987442,0.510462532,1.8625469784$

$\mathrm{C}, 1.1716758566,0.0657064991,1.1700663325$

C, $-1.2049458396,0.1054657733,-0.2240618087$

C, $1.2042657189,-0.1091983602,-0.2258044266$

C, $0.0026708515,0.0151190421,1.871927729$

C,-1.1683646271,-0.046814357,1.1743478368

C, $-0.0013429764,-0.0075322298,-0.921733325$

$\mathrm{N}, 2.4515843587,-0.4167871369,-0.9503189741$

O,-3.2496881626,-1.0853398613,1.2045002935

$\mathrm{N},-2.4186562167,-0.480072288,1.8776583149$

$\mathrm{N},-0.0034333809,-0.0196235688,-2.4074243314$

$\mathrm{N},-2.4542801148,0.4014702626,-0.9499298021$

$0,2.5343743671,0.2789946389,3.0521344394$

$0,3.2529714864,1.1046934307,1.1771424258$

$0,2.7120971521,0.2498715023,-1.9406475696$

$0,3.1387105691,-1.3178143018,-0.492390639$

$0,0.3208183487,-1.0621832255,-2.9461837439$

$0,-0.3291341207,1.0140674955,-2.9621648238$

O,-3.1401416438,1.3097948857,-0.5046905024

$0,-2.7176661658,-0.281107299,-1.928599657$

$0,-2.5255029027,-0.2291734339,3.0636252521$

$H F=-1254.5118987 \quad \mathrm{NImag}=0$

\section{$2,3,4,5,6-(\mathrm{CN})_{5}-\mathrm{C}_{6} \mathrm{H}$}

Brutoformula: $\mathrm{C} 11 \mathrm{H} 1 \mathrm{~N} 5$

0,1

$\mathrm{H}, 0 ., 0 .,-2.8816381615$

C,0.,0.,-1.7997767479

C,0.,0.,1.006530455

C, $-1.21213554,0 .,-1.108095938$

C, $1.21213554,0 .,-1.108095938$

C,1.2214304683,0.,0.3029638666

C, $-1.2214304683,0 ., 0.3029638666$

C,-2.4351643239,0.,-1.8475628605

C,2.4351643239,0.,-1.8475628605

C,2.4585121027,0.,1.0139588691

$\mathrm{C},-2.4585121027,0 ., 1.0139588691$

C, $0 ., 0 ., 2.4345118903$

$\mathrm{N},-3.4066026114,0 .,-2.4705385258$

$\mathrm{N},-3.4623909031,0 ., 1.5833988208$

$\mathrm{N}, 0 ., 0 ., 3.5884047431$

$\mathrm{N}, 3.4623909031,0 ., 1.5833988208$

$\mathrm{N}, 3.4066026114,0 .,-2.4705385258$

$\mathrm{HF}=-693.5947644 \mathrm{NImag}=0$

\section{$2,3,4,5,6-(\mathrm{CN})_{5}-\mathrm{C}_{6}^{-}$}

Brutoformula: C11N5(1-)

$-1,1$

C,-0.0617902002,-0.376402176,-2.613105232

C, $-0.0318741899,-0.3770993808,-1.1837948686$ 
C, $0.0289264784,-0.4075776994,1.596584153$ C, $-0.0365390615,-1.5860817706,-0.4460771497$ C, $0.002793422,0.8504683652,-0.4907758258$ $\mathrm{C}, 0.0329263445,0.8362224981,0.9187831811$ C, $-0.0063048463,-1.6736728505,0.9659649757$ C, $-0.074751359,-2.8252482185,-1.1793127865$ C, $0.0073673381,2.0862283057,-1.2041341231$ C,0.067168294,2.0739053064,1.6334315769 $\mathrm{N}, 0.0952492537,-0.3929812754,4.1923549776$ C, $0.06354737,-0.3924408283,3.0364542492$ $\mathrm{N},-0.0858643553,-0.3963821556,-3.768771604$ $\mathrm{N},-0.1076531308,-3.8265492605,-1.7567398345$ $\mathrm{N}, 0.0111475846,3.0872138969,-1.7820949963$ $\mathrm{N}, 0.0944324276,3.0644403221,2.2289501854$

$\mathrm{HF}=-693.0542289 \mathrm{NImag}=0$

\section{2,4,6- $\left(\mathrm{NO}_{2}\right)_{3}-\mathrm{C}_{6} \mathrm{H}_{2} \mathrm{CH}_{3}$}

Brutoformula: $\mathrm{C} 7 \mathrm{H} 5 \mathrm{~N} 3 \mathrm{O} 6$

0,1

C, $0.0699843103,-0.8095150296,-2.4171103725$ C, $-0.0069401647,-0.807724867,-0.9131551264$ C, $-0.0074089892,-0.8098915176,1.916082013$ C, $-0.067671215,-1.9861307331,-0.148195026$ C, $0.0493731248,0.3695326942,-0.1462073155$ C, $0.0615856715,0.3968304095,1.24060553$ C, $-0.0588533028,-2.0163524316,1.2386522232$ $\mathrm{N},-0.1567110451,-3.3123528224,-0.8111162665$ $\mathrm{N}, 0.0930195332,1.6989099853,-0.8073292472$ $\mathrm{H}, 0.1263496418,1.332078944,1.7787771723$ $\mathrm{H},-0.0882938937,-2.9542185228,1.7753268566$ $\mathrm{N},-0.0157066998,-0.8106902064,3.398488429$ $\mathrm{H}, 0.6257107299,0.0522459554,-2.7810197096$ $\mathrm{H},-0.932106202,-0.7622372906,-2.8487164674$ $\mathrm{H}, 0.5427724033,-1.7185533078,-2.7832850338$ $0,-0.8868265882,-3.4048123108,-1.786144001$ $0,0.4880151139,-4.2229601506,-0.3147759475$ O,-0.6302805341,1.8675154318,-1.7771858069 $0,0.8316334637,2.5373061727,-0.3146663448$ $0,-0.0776606097,-1.8960653782,3.9549485758$ $0,0.0400153568,0.2741285107,3.9567315952$

$\mathrm{HF}=-885.3033014 \mathrm{NImag}=0$

\section{2,4,6- $\left(\mathrm{NO}_{2}\right)_{3}-\mathrm{C}_{6} \mathrm{H}_{2} \mathrm{CH}_{2}^{-}$}

Brutoformula: $\mathrm{C} 7 \mathrm{H} 4 \mathrm{~N} 3 \mathrm{O} 6(1-)$

$-1,1$

O,-2.3336852914,0.4328297384,-2.4897951178 C, $0.2047927937,-0.0368751399,-1.2175031847$ C, $-0.1774460456,-0.0387161306,1.6668416844$ C, $1.3127707591,0.0115673672,-0.2273865447$ C,-1.1225570529,0.016298738,-0.5500419709 C,-1.2861658843,-0.0072323966,0.8114503597 C, $1.1158849731,-0.0117573828,1.1296982197$ $\mathrm{N}, 2.7088261698,0.0936686899,-0.6264011286$ $\mathrm{N},-2.366186602,0.1034289256,-1.2989348486$
$\mathrm{H},-2.2818010808,0.0158122841,1.2267902788$ $\mathrm{H}, 1.9687264045,0.0079876696,1.7904744744$ $\mathrm{N},-0.3647131388,-0.0338884834,3.0815514888$ $0,-1.5239877042,-0.033247621,3.5283130359$ $0,0.6384651829,-0.0376644248,3.8145059692$ C, $0.3818262163,-0.1867497944,-2.5535533946$ O,2.9889926203,0.4229647828,-1.7843596091 $0,3.5849175724,-0.1608126303,0.2143996567$ $0,-3.4320457356,-0.1469195698,-0.7153814204$ $\mathrm{H},-0.4642549637,-0.2340682281,-3.2161274444$ $\mathrm{H}, 1.3714891561,-0.2371545367,-2.9719698078$

$\mathrm{HF}=-884.7897276 \quad \mathrm{NImag}=0$

\section{2,3,4,5,6-( $\left.\mathrm{NO}_{2}\right)_{5}-\mathrm{C}_{6} \mathrm{CH}_{3}$}

Brutoformula: $\mathrm{C} 7 \mathrm{H} 3 \mathrm{~N} 5 \mathrm{O} 10$

0,1

$\mathrm{N}, 0.0008351436,2.494269643,-1.5525309352$ C, $-0.02023643,1.1945826225,-0.8233886643$ C, $0.0439221061,-1.1907724876,0.5592488475$ C, $0.0027380023,1.1746593749,0.564358077$ C, $-0.0423215847,-0.0005594007,-1.5291347358$ C, $-0.0211587495,-1.1983181097,-0.8298868705$ C, $0.0461083805,-0.0101875175,1.3095042615$ $\mathrm{N}, 0.0439411476,2.4668724039,1.3037454972$ $\mathrm{N},-0.0869673541,0.0042238561,-3.0190565085$ $\mathrm{N},-0.0924969208,-2.4927732872,-1.5655718186$ C, $0.0663671742,0.0118652379,2.8164768945$ $\mathrm{N}, 0.049379588,-2.4965419604,1.2775143578$ $\mathrm{H},-0.9096487292,0.3234035599,3.1947429286$ $\mathrm{H}, 0.8035295542,0.7313326238,3.1788738313$ $\mathrm{H}, 0.3194476498,-0.9592073976,3.2310636231$ $0,1.0273324683,3.1605653183,1.1199803695$ $0,-0.8892613777,2.6957181924,2.0476810309$ $0,0.8682409497,2.6216851719,-2.3930015963$ $0,-0.846936829,3.3025028415,-1.2294319484$ $0,0.7845165816,-0.6275665127,-3.5811392711$ $0,-0.990200474,0.6403465377,-3.5232481863$ $0,-1.0083678243,-2.6090021444,-2.3548449523$ $0,0.766712417,-3.3091556763,-1.2978306138$ $0,-0.965834296,-3.161765842,1.1842804768$ $0,1.0503594063,-2.7661770469,1.9115959054$

$\mathrm{HF}=-1294.3575164 \mathrm{NImag}=0$

\section{2,3,4,5,6- $\left(\mathrm{NO}_{2}\right)_{5}-\mathrm{C}_{6} \mathrm{CH}_{2}^{-}$}

Brutoformula: $\mathrm{C} 7 \mathrm{H} 2 \mathrm{~N} 5 \mathrm{O} 10(1-)$

$-1,1$

$\mathrm{N},-1.5678762597,-2.5074854381,0.0315971745$

C, $-0.8054536344,-1.2156831785,0.0048480769$ C, $0.5369186482,1.202150319,0.1318246245$ C, $0.5755673412,-1.2346728072,0.0891438835$ C, $-1.5461721103,-0.0266005334,-0.0689148373$ C,- $0.8157029205,1.1934524897,0.0305941979$ C, $1.3767997446,0.004218749,0.0381873506$ $\mathrm{N}, 1.2392326974,-2.50292642,0.3070105774$ 
$\mathrm{N},-2.974264893,-0.0196675875,-0.1649919264$ $\mathrm{N},-1.525099448,2.4984023813,-0.0911821797$ C,2.7242091409,0.0981104922,-0.0812452875 $\mathrm{N}, 1.2317783597,2.5056366688,0.214289$ $\mathrm{H}, 3.2135921883,1.0585016033,-0.0091711657$ $0,1.8921843018,2.721837457,1.2186254583$ $\mathrm{H}, 3.337355936,-0.7715411724,-0.2319553704$ $0,0.6543284949,-3.3681820895,0.9631291088$ $0,2.3701487339,-2.656959293,-0.1605800424$ O,-2.2219738838,-2.7327958529,1.0295552306 O,-1.4739815978,-3.2116128683,-0.9552117644 $0,-3.577394522,0.931154957,0.3450552785$ $0,-3.528148446,-0.9558575168,-0.7465067629$ $0,-2.0476429009,2.7295101719,-1.1647461711$ $0,-1.4856117953,3.2442050867,0.8718867628$ O,1.1214611017,3.2694345578,-0.7355768955

$H F=-1293.8615676 \quad \mathrm{NImag}=0$

\section{2,3,4,5,6-(CN)5- $\mathrm{C}_{6} \mathrm{CH}_{3}$}

Brutoformula: $\mathrm{C} 11 \mathrm{H} 1 \mathrm{~N} 5$

0,1

$\mathrm{H}, 0 ., 0 .,-2.8816381615$

C,0.,0.,-1.7997767479

C,0.,0.,1.006530455

C,-1.21213554,0.,-1.108095938

C,1.21213554,0.,-1.108095938

C, $1.2214304683,0 ., 0.3029638666$

C, $-1.2214304683,0 ., 0.3029638666$

C,-2.4351643239,0.,-1.8475628605

C,2.4351643239,0.,-1.8475628605

C,2.4585121027,0.,1.0139588691

C, $-2.4585121027,0 ., 1.0139588691$

C, $0 ., 0 ., 2.4345118903$

$\mathrm{N},-3.4066026114,0 .,-2.4705385258$

$\mathrm{N},-3.4623909031,0 ., 1.5833988208$

$\mathrm{N}, 0 ., 0 ., 3.5884047431$

$\mathrm{N}, 3.4623909031,0 ., 1.5833988208$

$\mathrm{N}, 3.4066026114,0 .,-2.4705385258$

$H F=-732.9263319 \quad$ NImag $=0$

\section{2,3,4,5,6-(CN)5- ${ }_{6} \mathrm{CH}_{2}^{-}$}

Brutoformula: C11N5(1-)

$-1,1$

C,-0.0617902002,-0.376402176,-2.613105232

C, $-0.0318741899,-0.3770993808,-1.1837948686$ C, $0.0289264784,-0.4075776994,1.596584153$

C,- $0.0365390615,-1.5860817706,-0.4460771497$ C, $0.002793422,0.8504683652,-0.4907758258$ C, $0.0329263445,0.8362224981,0.9187831811$ C, $-0.0063048463,-1.6736728505,0.9659649757$ C, $-0.074751359,-2.8252482185,-1.1793127865$ C, $0.0073673381,2.0862283057,-1.2041341231$ C, $0.067168294,2.0739053064,1.6334315769$ $\mathrm{N}, 0.0952492537,-0.3929812754,4.1923549776$
C, $0.06354737,-0.3924408283,3.0364542492$ $\mathrm{N},-0.0858643553,-0.3963821556,-3.768771604$ $\mathrm{N},-0.1076531308,-3.8265492605,-1.7567398345$ $\mathrm{N}, 0.0111475846,3.0872138969,-1.7820949963$ $\mathrm{N}, 0.0944324276,3.0644403221,2.2289501854$

$H F=-732.4246485 \quad N I m a g=0$

2,4,6- $\left(\mathrm{NO}_{2}\right)_{3}-\mathrm{C}_{6} \mathrm{H}_{2} \mathrm{OH}$

Brutoformula: $\mathrm{C} 6 \mathrm{H} 3 \mathrm{~N} 3 \mathrm{O} 7$

0,1

$\mathrm{N}, 0.0024492133,-0.8556502054,-1.9398235292$

C, $-0.0090712679,-0.9078774244,-0.4600451724$ C, $0.0220354117,-0.899884051,2.3221366264$ C, $0.021625876,-2.1560636502,0.2041084629$ C, $-0.0515986587,0.2922873075,0.2162617$ C, $-0.0205346243,0.282071867,1.6089957522$ C, $0.033447219,-2.1025833983,1.6244776953$ $0,0.000828095,-3.2700281451,-0.4981938158$ $\mathrm{H},-0.1072150085,1.2263969278,-0.3264155701$ $\mathrm{N},-0.0420661711,1.5656509598,2.3395719861$ $\mathrm{N}, 0.0555553629,-3.3386230865,2.410259498$ $\mathrm{H}, 0.0384975544,-0.8974689748,3.4030702133$ $\mathrm{H}, 0.0013079782,-4.0240225886,0.1377206477$ $0,-0.7504915377,-0.0457174172,-2.4610372671$ $0,0.779634774,-1.5923412576,-2.5192595159$ $0,0.0968965709,-3.262923431,3.6202654714$ $0,0.0292123798,-4.415071258,1.7879233395$ $0,-0.0743396136,2.5874803607,1.6695241318$ $0,-0.0261735175,1.5158256004,3.5604859389$

$\mathrm{HF}=-921.2303695 \mathrm{NImag}=0$

2,4,6-( $\left.\mathrm{NO}_{2}\right)_{3}-\mathrm{C}_{6} \mathrm{H}_{2} \mathrm{O}^{-}$

Brutoformula: $\mathrm{C} 6 \mathrm{H} 2 \mathrm{~N} 3 \mathrm{O} 7(1-)$

$-1,1$

$\mathrm{H}, 0.0289004383,0.2698921428,-2.1161371996$ $\mathrm{C}, 0.0077957873,0.2656508533,-1.0354531263$ C, $-0.0298454528,0.2931018228,1.7563302074$ C, $0.0356778482,-0.9227912097,-0.3518407101$ C, $-0.033787354,1.4767201976,-0.3322626736$ C,- $0.0576201364,1.4791010538,1.068507093$ C,- $0.0529879509,-1.0389021161,1.1181177047$ $\mathrm{N}, 0.1281552581,-2.1380152391,-1.1560180656$ $\mathrm{N},-0.0280442805,2.7204003211,-1.0492587085$ $\mathrm{H},-0.086872177,2.4165137801,1.6058342579$ O,-0.2116511042,-2.0934919354,1.7211143694 $\mathrm{N},-0.0067622064,0.3825064053,3.2136317156$ $0,0.0028490842,2.688805928,-2.286483877$ $0,-0.4342106219,-2.145584943,-2.257270918$ $0,0.7773675389,-3.0772392667,-0.715519641$ $0,-0.6161506382,1.3184845319,3.7443722571$ $0,0.6339152807,-0.4539849067,3.8361303574$ $0,-0.0567288075,3.7755349111,-0.4022481428$

$H F=-920.7395737 \quad \mathrm{NImag}=0$ 


\section{2,3,4,5,6-( $\left(\mathrm{NO}_{2}\right)_{5}-\mathrm{C}_{6} \mathrm{OH}$}

Brutoformula: $\mathrm{C} 6 \mathrm{H} 1 \mathrm{~N} 5 \mathrm{O} 11$

0,1

$\mathrm{N},-2.8378225693,0.0306852214,-0.9129803132$

C,-1.4066519377, $0.0336757542,-0.5057226869$

C, $1.2733380806,-0.0180108795,0.2191206593$

C, $-0.4384286806,-0.0333863116,-1.52754721$

C, $-1.0493299854,0.0511126867,0.8234436339$

C, $0.2965473444,0.0224365517,1.1977897829$

C, $0.9214063801,-0.0262047197,-1.1356139997$

O,-0.8639502069,-0.1057179123,-2.7730802678

$\mathrm{N},-2.119979121,0.0391323269,1.8648989062$

$\mathrm{N}, 0.6666766909,0.0667659647,2.6376935983$

$\mathrm{N}, 1.9531764543,0.0550275127,-2.1792238979$

$\mathrm{N}, 2.7102055319,-0.0829203678,0.6402193441$

$\mathrm{H},-0.0881403004,-0.2581707348,-3.3595433832$

O,-3.4893263023,-0.9398683848,-0.5713438216

O,-3.2138092779, $0.9873727614,-1.5554124576$

$0,-2.9265951347,0.9439792728,1.8122080389$

$0,-2.0831135025,-0.8874030295,2.6478498354$

$0,0.1127426445,0.9228228383,3.3007851486$

$0,1.4858761225,-0.7469117657,3.0155502819$

$0,3.148875186,0.9089482982,1.1823248761$

$0,3.2768009453,-1.1293576926,0.4135100475$

$0,3.0403831126,0.4960362508,-1.8860067821$

O,1.6288954445,-0.3166118895,-3.3161686251

$H F=-1330.278537 \quad \mathrm{NImag}=0$

\section{2,3,4,5,6-( $\left(\mathrm{NO}_{2}\right)_{5}-\mathrm{C}_{6} \mathrm{O}^{-}$}

Brutoformula: C6N5O11(1-)

$-1,1$

$\mathrm{N},-2.4857886899,-0.0446602852,-1.7187285394$

C,-1.2154371135,0.0081341572,-0.975062018

C, $1.2026466574,0.0202028972,0.3911411548$

C, $-0.0043016546,0.0673812527,-1.7954916849$

C, $-1.2058347771,-0.0481387387,0.3913956037$

C, $-0.0022062783,0.0131612043,1.1325919666$

C, $1.2084481516,0.0777421093,-0.975543549$

O,-0.0036566973,0.0277351062,-3.0200605124

$\mathrm{N},-2.524041921,-0.2207226579,1.0807346182$

$\mathrm{N},-0.0102055477,0.2806858708,2.547267687$

$\mathrm{N}, 2.4795026487,0.1028202354,-1.7193202506$

$\mathrm{N}, 2.5293531168,-0.0714535169,1.079872897$

$0,2.6756376275,1.0696572817,-2.4326363759$

$0,-3.1801493366,-1.0458994741,-1.5860866099$

O,-2.7416466539,0.9089729403,-2.4307685879

$0,-3.2895051089,0.726066605,1.0478303105$

O,-2.7396107224,-1.3159392055,1.5574466202

$0,-1.0992786431,0.3824323883,3.1128731327$

$0,1.0715995287,0.4199253029,3.1186135946$

$0,3.229836342,0.9248080956,1.0566698928$

$0,2.8174674551,-1.1542740844,1.5459652054$

$0,3.2351631826,-0.8528437373,-1.5853000183$
$H F=-1329.8147153 \quad N I m a g=0$

\section{2,3,4,5,6-(CN) $)_{5}-\mathrm{C}_{6} \mathrm{OH}$}

Brutoformula: $\mathrm{C} 11 \mathrm{H} 1 \mathrm{~N} 5 \mathrm{O} 1$

0,1

C, $0.0179997675,-0.3773527062,-1.9685728972$

C, $-0.0095887131,-0.3687618422,-0.5409765868$

C, $-0.0636729815,-0.3562637148,2.2631640418$

C, $-0.0497952426,-1.5865430735,0.1602182984$

C, $0.0039178023,0.8615860519,0.151206853$

C, $-0.023404752,0.8610552736,1.5573776493$

C,-0.077025937,-1.5830016442,1.5698900999

C, $-0.0640222129,-2.8344217239,-0.5287179343$

C, $0.0448610132,2.093140178,-0.5669696519$

C, $-0.0103208209,2.0917430228,2.2800831795$

O,-0.114661621,-2.7645119581,2.1836587983

C, $-0.0929197854,-0.4094856484,3.6864585056$

$\mathrm{H},-0.131936411,-2.6741608108,3.1489465566$

$\mathrm{N},-0.0763025703,-3.8468821166,-1.0824956488$

$\mathrm{N}, 0.0402964211,-0.3841392598,-3.1222978312$

$\mathrm{N}, 0.0780123084,3.0912025063,-1.1457135053$

$\mathrm{N},-0.0002365699,3.079863731,2.8763047637$

$\mathrm{N},-0.1209150219,-0.5639219631,4.8314665104$

$H F=-768.8447273 \quad N I m a g=0$

\section{$2,3,4,5,6-(\mathrm{CN})_{5}-\mathrm{C}_{6} \mathrm{O}$}

Brutoformula: C11N5O1(1-)

$-1,1$

O,-0.0000000053,0.198863122,-2.748106192

$\mathrm{C}, 0.0000000436,0.1988633622,-1.51748037$

C, $-0.0000000041,0.1988639409,1.4039538701$

C, $0.0000000002,-1.0344346318,-0.7183758891$

C, $0.0000000111,1.4321616763,-0.7183763742$

C, $-0.0000000092,1.4155103052,0.6766867159$

C, $-0.0000000087,-1.0177827091,0.6766871949$

C, $-0.0000000037,-2.2656314864,-1.4309732902$

C, $0.0000000123,2.6633582481,-1.4309742636$

C, $0.000000008,2.6522773024,1.4001287501$

C, $0.0000000112,-2.2545494196,1.4001297205$

C, $-0.000000008,0.198864223,2.8237294658$

$\mathrm{N}, 0.0000000246,3.664785148,-2.0103248491$

$\mathrm{N}, 0.000000051,-3.2512049249,1.9837070069$

$\mathrm{N},-0.0000000047,-3.267058618,-2.0103234753$

$\mathrm{N}, 0.0000000092,0.198864453,3.981632483$

$\mathrm{N}, 0.0000000085,3.6489330445,1.9837056323$

$\mathrm{HF}=-768.3815553 \mathrm{~N} I \mathrm{mag}=0$

\section{2,4,6- $\left(\mathrm{NO}_{2}\right)_{3}-\mathrm{C}_{6} \mathrm{H}_{2} \mathrm{NH}_{2}$}

Brutoformula: $\mathrm{C} 6 \mathrm{H} 4 \mathrm{~N} 4 \mathrm{O} 6$

0,1

$\mathrm{H},-0.0056691272,-0.6484880116,-2.1670091588$

C, $0.013728229,-0.6464658205,-1.0864587854$

C, $0.0622433871,-0.6367762838,1.6976720098$

C, $0.053387015,-1.8471578794,-0.4005284234$ 
C, $-0.0007591957,0.5469463079,-0.3830900505$ C, $0.0228603667,0.5581984027,1.0020039973$ C, $0.0815900385,-1.917406339,1.0379931717$ $\mathrm{N}, 0.065553973,-3.0584005296,-1.236274089$ $\mathrm{N},-0.0415856391,1.8209979382,-1.1176068183$ $\mathrm{H}, 0.0106875619,1.4926599774,1.5448195518$ $\mathrm{N}, 0.1217351957,-3.0709705509,1.7038082311$ $\mathrm{N}, 0.0840802023,-0.5198616466,3.1646682334$ $\mathrm{H}, 0.1349255866,-3.9311160928,1.1718854189$ $\mathrm{H}, 0.1387282621,-3.0393200669,2.7146314225$ $0,0.108080211,-4.1595518415,-0.6770627045$ $0,0.0332788149,-2.9202266691,-2.4470605956$ $0,-0.0622838756,1.7643872064,-2.3398087462$ $0,-0.0519840613,2.8506401885,-0.4563852811$ $0,0.0638627438,0.5975877755,3.6514842143$ $0,0.1216617564,-1.5556168677,3.8376685979$

$\mathrm{HF}=-901.3733938 \quad \mathrm{NImag}=0$

\section{2,4,6- $\left(\mathrm{NO}_{2}\right)_{3}-\mathrm{C}_{6} \mathrm{H}_{2} \mathrm{NH}^{-}$}

Brutoformula: $\mathrm{C} 6 \mathrm{H} 3 \mathrm{~N} 4 \mathrm{O} 6(1-)$

$-1,1$

$\mathrm{H}, 0.0761919826,-0.6544101248,-2.1658065307$ C, $0.0462219992,-0.6583497342,-1.0863807205$ C, $0.033051196,-0.6342973821,1.6889826281$ C, $0.0371163188,-1.8698944184,-0.4151917729$ C, $0.0164021162,0.5445040133,-0.3927536818$ C, $0.0091609432,0.5475999452,1.0244751383$ C,-0.0420840292,-1.9672924822,1.0628960104 $\mathrm{N}, 0.0688677005,-3.0477760542,-1.2413674778$ $\mathrm{N}, 0.0153108213,1.7849862767,-1.1049088843$ $\mathrm{H}, 0.0082507998,1.486468239,1.5597314702$ $\mathrm{N},-0.2030374257,-3.0044344027,1.8104687267$ $\mathrm{N}, 0.1273252316,-0.5529848687,3.1554963863$ $\mathrm{H},-0.2564808814,-3.8364731434,1.2294809142$ $0,1.0174958322,-1.1787228773,3.7096836567$ O,-0.0140128404,-4.1634261362,-0.6991869959 $0,0.1795824487,-2.9253448509,-2.4695425282$ $0,0.0221089785,1.7654685651,-2.3441493154$ $0,0.0070146761,2.8382843883,-0.4480343965$ $0,-0.6714252729,0.1826415094,3.7369405251$

$\mathrm{HF}=-900.8500673 \mathrm{NImag}=0$

\section{2,3,4,5,6-( $\left.\mathrm{NO}_{2}\right)_{5}-\mathrm{C}_{6} \mathrm{NH}_{2}$}

Brutoformula: $\mathrm{C} 6 \mathrm{H} 2 \mathrm{~N} 6 \mathrm{O} 10$

0,1

$\mathrm{N},-0.1191962093,-0.0090670381,-2.8482366528$

C, $-0.0309671198,-0.0041924272,-1.3671771797$ C, $0.137938445,0.0054505667,1.4844871352$ C, $0.0610572838,-1.1974087912,-0.6607205821$ C, $-0.0393233787,1.1937396675,-0.6628683195$ C, $0.0665031246,1.2127815732,0.7199476511$ C, $0.119793757,-1.2071886293,0.7250329857$ $\mathrm{N}, 0.0873331224,-2.4833505297,-1.4292077218$ $\mathrm{N},-0.1576840424,2.4747791919,-1.4308057401$
$\mathrm{N}, 0.2073664661,2.5096373999,1.4078363187$ $\mathrm{N}, 0.0629427628,-2.4992984529,1.4337043718$ $\mathrm{N}, 0.2167739462,0.0101613135,2.8199886315$ $0,1.1121078838,-3.1260808459,-1.3466161493$ $0,-0.9104461901,-2.739885234,-2.0680047593$ $0,0.6890319765,-0.7032210589,-3.4355164917$ O,-0.9915579252,0.6818117179,-3.3397764305 $0,-1.1654635574,3.118390373,-1.2298889398$ $0,0.756244063,2.727068865,-2.1860744775$ $0,0.8147714403,3.3888401176,0.8296349856$ $0,-0.2820468995,2.6085088475,2.5311467128$ $0,0.6804001059,-2.5893440863,2.4928731428$ $\mathrm{O},-0.6057761828,-3.3835653212,0.9366584201$ $\mathrm{H}, 0.4499402542,-0.8459470194,3.3026803596$ $\mathrm{H}, 0.0402468736,0.8693898002,3.3208927292$

$H F=-1310.4184358 \quad N I m a g=0$

\section{2,3,4,5,6-( $\left(\mathrm{NO}_{2}\right)_{5}-\mathrm{C}_{6} \mathrm{NH}^{-}$}

Brutoformula: $\mathrm{C} 6 \mathrm{H} 1 \mathrm{~N} 6 \mathrm{O} 10(1-)$

$-1,1$

$\mathrm{N},-0.0190876276,0.2390918194,-2.6872232675$ C, $-0.0037479776,0.1250878068,-1.2495901844$ C, $-0.0349712657,-0.0629423792,1.6614921073$ C, $-1.2261117853,0.0081092013,-0.5263460631$ C, $1.1850683369,0.0522134369,-0.5234145777$ C, $1.2079456587,-0.0702525648,0.8618343267$ C, $-1.2342033548,-0.1307833818,0.820646056$ $\mathrm{N},-2.5294080814,0.1782275981,-1.2281548741$ $\mathrm{N}, 2.4757132719,0.093073822,-1.2870005664$ $\mathrm{N}, 2.4638627102,-0.2830883482,1.5382039744$ $\mathrm{N},-2.5176847251,-0.230095277,1.5534531369$ $\mathrm{N},-0.1982275709,-0.0132886655,2.9365157356$ O,-3.3145195026,-0.7513166551,-1.1780282378 O,-2.7184738539,1.259143037,-1.7549898049 $0,-0.9421727181,-0.3149226179,-3.2864532283$ $0,0.8810056082,0.874222768,-3.2324206305$ 0,3.1408272845,1.1032552256,-1.1676594069 $0,2.7380279642,-0.879365569,-1.9658973139$ $0,3.3832878118,-0.8302758828,0.9292630388$ $0,2.5551599693,0.076151028,2.7192426206$ $0,-3.2082152854,0.7770356341,1.6120010674$ $0,-2.7805310586,-1.3090130045,2.0472820991$ $\mathrm{H}, 0.6964561933,0.0897329674,3.4072439926$

$H F=-1309.9248884 \quad N I m a g=0$

\section{2,3,4,5,6-(CN) $)_{5}-\mathrm{C}_{6} \mathrm{NH}_{2}$}

Brutoformula: $\mathrm{C} 11 \mathrm{H} 2 \mathrm{~N} 6$

0,1

$\mathrm{N}, 0 ., 0 .,-2.9353965287$

C, $0 ., 0 .,-1.5872580033$

C, $0 ., 0 ., 1.2749382479$

C, $-1.2207816368,0 .,-0.8513516618$

C, $1.2207816368,0 .,-0.8513516618$

C, $1.2154142847,0 ., 0.5548367974$ 
C, $-1.2154142847,0 ., 0.5548367974$

C,-2.4411483516,0.,-1.5912317294

C,2.4411483516,0.,-1.5912317294

C,2.4619944042,0.,1.2603642549

C,-2.4619944042,0.,1.2603642549

C,0.,0.,2.7033106698

$\mathrm{N},-3.3774048483,0 .,-2.2829391333$

$\mathrm{N},-3.4782166616,0 ., 1.8245477961$

$\mathrm{N}, 0 ., 0 ., 3.8662775659$

$\mathrm{N}, 3.4782166616,0 ., 1.8245477961$

$\mathrm{N}, 3.3774048483,0 .,-2.2829391333$

$\mathrm{H},-0.8665199545,0 .,-3.4580229795$

$\mathrm{H}, 0.8665199545,0 .,-3.4580229795$

$\mathrm{HF}=-748.8135932 \mathrm{NImag}=0$

\section{2,3,4,5,6-(CN) $)_{5}-\mathrm{C}_{6} \mathrm{NH}^{-}$}

Brutoformula: $\mathrm{C} 11 \mathrm{H} 1 \mathrm{~N} 6(1-)$

$-1,1$

C,-0.1292993891, $0.0000000008,2.8045308578$

C, $-0.0812348938,-0.0000000133,1.3852953096$

C, $0.0094685342,-0.0000000112,-1.5467850259$

C, $-1.2776796001,-0.0000000144,0.6054675311$

C, $1.1573043363,-0.0000000231,0.6930808751$

C, $1.219782576,-0.0000000185,-0.7032843388$

C,- $1.2492269365,-0.0000000094,-0.7865674431$

C,-2.5409996217,-0.0000000081, 1.2891893377

C,2.3774136937,-0.000000008,1.4512317505

C,2.4741066278,0.0000000015,-1.3734540085

C,-2.4693595999, $0.000000016,-1.526620316$

$\mathrm{N},-0.032837209,-0.0000000042,-2.843264067$

$\mathrm{N},-0.1673951863,0.0000000237,3.9712301486$

$\mathrm{N},-3.5582655551,-0.0000000038,1.8533637733$

$\mathrm{N},-3.4786298152,0.0000000368,-2.1091297161$

$\mathrm{N}, 3.3683535033,0.0000000193,2.0606911344$

$\mathrm{N}, 3.4682014624,0.0000000362,-1.9853719807$ $\mathrm{H}, 0.9102970731,-0.0000000204,-3.239603821$

$\mathrm{HF}=-748.3155139 \mathrm{NImag}=0$

\section{2,4,6- $-\mathrm{Tf}_{3}-\mathrm{C}_{6} \mathrm{H}_{2} \mathrm{CH}_{3}$}

Brutoformula: $\mathrm{C} 10 \mathrm{H} 5 \mathrm{~F} 906 \mathrm{S3}$

0,1

C,1.2733312452,0.,-2.6298022399

C, $0.7108013444,0 .,-1.2317845652$

C, $-0.275079003,0 ., 1.4303057703$

C, $0.448228541,-1.1915381959,-0.5158642569$ C, $0.448228541,1.1915381959,-0.5158642569$

C, $-0.0381212001,1.2068614868,0.7895960163$

C, $-0.0381212001,-1.2068614868,0.7895960163$

$\mathrm{S}, 0.7529897496,-2.8639901654,-1.1866996925$

S, $0.7529897496,2.8639901654,-1.1866996925$

$\mathrm{H},-0.2258510385,2.1476330526,1.2924602284$

$\mathrm{H},-0.2258510385,-2.1476330526,1.2924602284$

S, $-0.9347371776,0 ., 3.1071413832$

$\mathrm{H}, 0.9788951624,-0.8829280011,-3.1855996607$
$\mathrm{H}, 2.3658568348,0 .,-2.5770535368$

$\mathrm{H}, 0.9788951624,0.8829280011,-3.1855996607$

$0,1.8523672145,-2.8499553214,-2.1394774494$

$0,0.7105385281,-3.7940137759,-0.0668969609$

C,-0.8408025291,-3.2164165823,-2.178924708

O,0.7105385281,3.7940137759,-0.0668969609

$\mathrm{O}, 1.8523672145,2.8499553214,-2.1394774494$

C,-0.8408025291,3.2164165823,-2.178924708

O,-1.5804740174,1.2823426791,3.3469669728

$0,-1.5804740174,-1.2823426791,3.3469669728$

C, $0.6550776138,0 ., 4.1607566004$

$\mathrm{F}, 1.3777104056,1.0868231106,3.8848433562$

$F, 1.3777104056,-1.0868231106,3.8848433562$

$F, 0.3135477916,0 ., 5.441443486$

F,-1.8979320955,3.1087502311,-1.3754137972

$\mathrm{F},-0.9679863302,2.3436172864,-3.1834590728$

F,- $0.7656311409,4.4463825382,-2.6656121503$

$\mathrm{F},-0.9679863302,-2.3436172864,-3.1834590728$

$\mathrm{F},-1.8979320955,-3.1087502311,-1.3754137972$

$F,-0.7656311409,-4.4463825382,-2.6656121503$

$H F=-2928.9054143 \quad \mathrm{NImag}=1$

\section{2,4,6- $-\mathrm{Tf}_{3}-\mathrm{C}_{6} \mathrm{H}_{2} \mathrm{CH}_{2}^{-}$}

Brutoformula: C10H4F9O6S3(1-)

$-1,1$

C, $0.2343982214,-0.0759971046,-2.8330681483$

C, $0.0050644994,0.0090199418,-1.5054217677$

C, $-0.4952040092,0.1923438739,1.3715689789$

C, $-0.587880624,-1.078501174,-0.6808713676$

C, $0.3083760902,1.2031451251,-0.6705211031$

C, $0.0691299214,1.2812881342,0.6772118689$

C,- $0.8121738511,-0.9874965269,0.668304417$

S, $-1.0292983842,-2.6579719489,-1.3604502537$

S, $0.9560958456,2.7061017702,-1.3565681466$

$\mathrm{H}, 0.3042372136,2.1930083799,1.2115037554$

$\mathrm{H},-1.2542547043,-1.8235003494,1.1952184846$

S, $-0.7527551954,0.286719411,3.0812600434$

$\mathrm{H},-0.0493486058,-0.9509653374,-3.3941909448$ $\mathrm{H}, 0.7056449277,0.7294383052,-3.3716808409$

$\mathrm{F}, 1.2508130486,-3.635400208,-0.3196203316$

$0,-1.7620693803,-3.4405265239,-0.3625583465$

$0,-1.5241700655,-2.5722851182,-2.7340137136$

C, $0.6300911996,-3.5961912788,-1.5048264412$

$0,1.3382579727,3.6203572142,-0.278096753$

$0,1.8659799266,2.4748475211,-2.4780014305$

C,- $0.5588211855,3.5729985173,-2.136278949$

O,-0.8046608627, $1.6834094948,3.5185911641$

$0,-1.7507284051,-0.6963656399,3.5078042059$

C, $0.8580503752,-0.3578836132,3.8698826761$

$F, 1.9036422247,0.3948310572,3.4998840862$

$\mathrm{F}, 1.0967740739,-1.6232911869,3.4966376774$

F, $0.7609814382,-0.3214460763,5.205974646$

$\mathrm{F},-1.5226787987,3.7408355161,-1.2234277114$

F,-1.059341034,2.8795106864,-3.1628554117

F,-0.178736853,4.7786994617,-2.5819348264 
$F, 1.4490222176,-3.0312996731,-2.3964878972$ $F, 0.3775223054,-4.8532639848,-1.8953256467$

$H F=-2928.4079941 \quad \mathrm{NImag}=0$

\section{4-Tf- $\mathrm{C}_{6} \mathrm{H}_{4} \mathrm{OH}$}

Brutoformula: $\mathrm{C} 7 \mathrm{H} 5 \mathrm{~F} 3 \mathrm{O} 3 \mathrm{~S} 1$

0,1

0,-0.8829349683,0.62062135,-4.4489554074

C, $-0.5016571934,0.5612525591,-3.1477944843$

C, $0.1642338716,0.5043977918,-0.4645857794$

C,0.7016246138,-0.0277073934,-2.7422722145

C,-1.369497138,1.1262725091,-2.2059291955

C, $-1.0368384195,1.0995093015,-0.8616132129$

C, $1.0378263008,-0.0568454577,-1.3959118492$

$\mathrm{H}, 1.3752694969,-0.4547461323,-3.4782616264$

$\mathrm{H},-2.2891447439,1.584610904,-2.5469018958$

$\mathrm{H},-1.6899762414,1.5462906115,-0.1227422646$

$\mathrm{H}, 1.9720708103,-0.4953863952,-1.0688309354$ $\mathrm{S}, 0.5970691035,0.4729465726,1.26126569$

$\mathrm{H},-0.2182762542,0.2121021128,-5.0147367659$ $0,2.0371338104,0.2805909357,1.397833601$ O,-0.1271254143,1.525400767,1.9657369797 C,- $0.1799457837,-1.1566618996,1.8692184468$ F,0.3304953902,-2.1933332565,1.196017274 F,-1.5041650132,-1.1354249103,1.6867603414 F,0.0832125503,-1.301711294,3.1647369174

$H F=-1193.328087 \quad \mathrm{NImag}=0$

\section{4-Tf- $\mathrm{C}_{6} \mathrm{H}_{4} \mathrm{O}$}

Brutoformula: C7H4F3O3S1(1-)

$-1,1$

$0,0.7492684563,-0.4058260639,-4.5227989877$ C, $0.3846167965,-0.3448139307,-3.3269060735$ C, $-0.4620028954,-0.2083276487,-0.5875960238$ C, $-0.1720299809,0.8641837399,-2.7357912684$ C, $0.480434796,-1.4781810459,-2.4173970211$ C, $0.0811588707,-1.410550006,-1.1089530239$ C, $-0.5710055274,0.9298862278,-1.4271151805$ $\mathrm{H},-0.2685373133,1.7280179096,-3.3856024036$ $\mathrm{H}, 0.8816059251,-2.4008289827,-2.8243632027$ $\mathrm{H}, 0.1578764402,-2.2764236446,-0.460612101$ $\mathrm{H},-0.9904579555,1.8437693436,-1.021017828$ S, $-0.885278375,-0.1009440567,1.0726021947$ F, $0.4490710626,0.5667937391,3.3201280561$ O,-1.8067773075,1.0149697881,1.3289534943 $0,-1.1257710053,-1.4267140214,1.6597159496$ C, $0.6890082423,0.4637394183,1.9997340487$ $F, 1.1084285011,1.6634954384,1.5626927374$ $F, 1.6946253192,-0.4118989406,1.8313063204$

$H F=-1192.8072826 \quad N I m a g=0$

\section{$\mathrm{C}_{6} \mathrm{~F}_{5} \mathrm{OH}$}

Brutoformula: C6H1F5O1
0,1

O,1.0865939053,-0.000000001,2.2772924184 C, $0.5217051646,0.0000000048,1.0486469552$ C, $-0.5921874697,0.0000000057,-1.5306118813$ C, $-0.8583140167,0.0000000037,0.8527138409$ C, $1.33929471,0.0000000004,-0.082554149$ C, $0.7883632032,0.0000000053,-1.3586060086$ C, $-1.4212306006,-0.0000000006,-0.4152773332$ F,-1.6498844225,-0.0000000061,1.9464870176 $\mathrm{F}, 2.6681945717,-0.0000000007,0.06022041$ $F, 1.5888437285,-0.0000000088,-2.427167479$ F,-2.7483949554,-0.0000000019,-0.5628366197 $\mathrm{F},-1.1177386605,-0.0000000003,-2.7593334944$ $\mathrm{H}, 0.3947548412,-0.0000000005,2.9510263221$

$\mathrm{HF}=-803.85687 \mathrm{NImag}=0$

$\mathrm{C}_{6} \mathrm{~F}_{5} \mathrm{O}^{-}$

Brutoformula: C6F5O1(1-)

$-1,1$

$0,0 ., 0.0000000008,-2.7603665573$

C, $0 .,-0.0000000007,-1.5043016764$

C,0.,-0.0000000058,1.4073576827

C,-1.192538889,0.0000000008,-0.6930578054

C, $1.1925388889,-0.0000000032,-0.6930578053$

C, $1.1920913053,-0.0000000051,0.6893133912$

C,-1.1920913051,-0.0000000033,0.689313391

$\mathrm{F},-2.3974027241,-0.0000000054,-1.3249377124$

$F, 2.397402724,0.000000006,-1.3249377124$

$F, 2.3684862123,0.0000000065,1.371655316$

$\mathrm{F},-2.3684862113,0.0000000151,1.3716553169$

$\mathrm{F},-0.0000000012,-0.0000000056,2.7713632985$

$\mathrm{HF}=-803.3300095 \mathrm{~N} / \mathrm{mag}=0$

\section{4- $\mathrm{NO}_{2}-\mathrm{C}_{6} \mathrm{H}_{4} \mathrm{OH}$}

Brutoformula: $\mathrm{C} 6 \mathrm{H} 5 \mathrm{~N} 1 \mathrm{O} 3$

0,1

$\mathrm{N},-0.1382053874,0 ., 2.1531725637$

C, $-0.0470029345,0 ., 0.6873413124$

C, $0.1244014475,0 .,-2.0716210845$

C, $-1.2177747711,0 .,-0.0708850181$

C, $1.2077614589,0 ., 0.0847300537$

C, $1.2924614049,0 .,-1.3000519822$

C, $-1.1311205266,0 .,-1.4529399509$

$\mathrm{H},-2.1758401449,0 ., 0.4303906264$

$\mathrm{H}, 2.0958992671,0 ., 0.7014316639$

$\mathrm{H}, 2.2644570585,0 .,-1.7828505121$

$\mathrm{H},-2.020995977,0 .,-2.0694584142$

$\mathrm{O}, 0.1446933423,0 .,-3.4297675613$

$0,0.9095187679,0 ., 2.7915312425$

$0,-1.2568325159,0 ., 2.6559462731$

H,1.0525242799,0.,-3.7528409285

$H F=-512.1240076 \quad \mathrm{NImag}=0$ 


\section{4- $\mathrm{NO}_{2}-\mathrm{C}_{6} \mathrm{H}_{4} \mathrm{O}^{-}$}

Brutoformula: C6H4N1O3(1-)

$-1,1$

$\mathrm{N},-0.1222106457,0 ., 2.0732310749$

C, $-0.039086631,0 ., 0.6630507489$

C,0.1299807876,0.,-2.2046389899

C,-1.2153844806,0.,-0.12335141

C, $1.2214555721,0 ., 0.0203061884$

C,1.306641266,0.,-1.347103911

C,-1.1393192352,0.,-1.491295833

$\mathrm{H},-2.1713343658,0 ., 0.3855081277$

$\mathrm{H}, 2.1110267726,0 ., 0.6379142164$

$\mathrm{H}, 2.2727653201,0 .,-1.8419082468$

$\mathrm{H},-2.0406126531,0 .,-2.0961821461$

$0,0.2036838135,0 .,-3.4558063097$

$0,0.9310626175,0 ., 2.7483784928$

O,-1.2475082093,0.,2.6199590375

$H F=-511.599737 \quad \mathrm{NImag}=0$

\section{2-Cl-4- $\mathrm{NO}_{2}-\mathrm{C}_{6} \mathrm{H}_{3} \mathrm{OH}$}

Brutoformula: $\mathrm{C} 6 \mathrm{H} 4 \mathrm{Cl} 1 \mathrm{~N} 1 \mathrm{O} 3$

0,1

$\mathrm{Cl},-1.5292378302,0 ., 2.4433200245$

C,-0.3717745959,0.,1.1206435301

C, $1.5066031853,0 .,-0.9310201909$

C, $-0.8111961804,0 .,-0.1914739032$

C, $0.998555193,0 ., 1.4326460686$

C, $1.9291486888,0 ., 0.3872221593$

C, $0.1403935253,0 .,-1.2061913508$

$\mathrm{H},-1.864600152,0 .,-0.4329325992$

$\mathrm{O}, 1.4631733498,0 ., 2.6969433864$

$\mathrm{H}, 2.9821699398,0 ., 0.638717365$

$\mathrm{N},-0.3170319343,0 .,-2.6046070775$

$\mathrm{H}, 2.2148904207,0 .,-1.747642717$

$0,0.5389275688,0 .,-3.4814741263$

O,-1.5255754505,0.,-2.8094639375

$\mathrm{H}, 0.7212238034,0 ., 3.3186666182$

$\mathrm{HF}=-971.7453619 \mathrm{NImag}=0$

\section{2-Cl-4- $\mathrm{NO}_{2}-\mathrm{C}_{6} \mathrm{H}_{3} \mathrm{O}^{-}$}

Brutoformula: $\mathrm{C} 6 \mathrm{H} 3 \mathrm{Cl} 1 \mathrm{~N} 1 \mathrm{O} 3(1-)$

$-1,1$

$\mathrm{Cl},-1.554026835,0 ., 2.4630437742$

C, $-0.3568512123,0 ., 1.1591520448$

C, $1.5304731159,0 .,-0.8948824932$

C, $-0.7893733818,0 .,-0.1384620234$

C, $1.0536210011,0 ., 1.5483231002$

C,1.9563488365,0.,0.4065335306

C, $0.1501720508,0 .,-1.1923763515$

$\mathrm{H},-1.844027347,0 .,-0.3763410204$

$\mathrm{O}, 1.4508802411,0 ., 2.7280139212$

$\mathrm{H}, 3.0150022842,0 ., 0.6436665763$

$\mathrm{N},-0.2964496694,0 .,-2.5368559295$

$\mathrm{H}, 2.235129199,0 .,-1.7165413147$
$0,0.5490342927,0 .,-3.4556803061$

O,-1.5222698733,0.,-2.7716165829

$\mathrm{HF}=-971.2274488 \mathrm{~N} / \mathrm{mag}=0$

\section{4- $\mathrm{NC}_{5} \mathrm{~F}_{4} \mathrm{OH}$}

Brutoformula: $\mathrm{C} 5 \mathrm{H} 1 \mathrm{~F} 4 \mathrm{~N} 1 \mathrm{O} 1$

0,1

O,-2.4757899586,-0.0220015343,-0.0578418735

C,-1.134721174,-0.0007747442,-0.0264875845

$\mathrm{N}, 1.6518691409,-0.0146436745,0.0383339112$

C,-0.3932739522,1.1826223671,-0.0099450402

C,- $-0.4182537577,-1.2004714524,-0.0091546756$

C, $0.9672814732,-1.1347263356,0.0230129802$

C, $0.9884050004,1.1155536701,0.0222291073$

$\mathrm{F},-1.0638220053,2.3536068829,-0.0259054489$

F,-1.0636614594,-2.3699710362,-0.0232485207

$F, 1.6588438324,-2.2748650722,0.0400783095$

$\mathrm{F}, 1.6914482101,2.2493851489,0.0381802271$

$\mathrm{H},-2.8186670559,0.8818936549,-0.0654722163$

$H F=-720.6615685 \quad N I m a g=0$

\section{4- $\mathrm{NC}_{5} \mathrm{~F}_{4} \mathrm{O}^{-}$}

Brutoformula: C5F4N1O1(1-)

$-1,1$

O,-2.5165510146,0.,-0.0525929118

C,-1.2697622167,0.,-0.0265707506

$\mathrm{N}, 1.6354006015,0 ., 0.0341478336$

C, $-0.4449212743,1.1888619985,-0.0092753516$

C,- $0.4449212743,-1.1888619985,-0.0092753516$

C, $0.9244434256,-1.1137746979,0.0193364494$

C, $0.9244434256,1.1137746979,0.0193364494$

$\mathrm{F},-1.0583763989,2.4028634848,-0.022068237$

$\mathrm{F},-1.0583763989,-2.4028634848,-0.022068237$

$F, 1.6444270318,-2.2718805099,0.0343126696$

$F, 1.6444270318,2.2718805099,0.0343126696$

$H F=-720.14765 \quad N I m a g=0$

\section{3-Tf- $\mathrm{C}_{6} \mathrm{H}_{4} \mathrm{OH}$}

Brutoformula: $\mathrm{C} 7 \mathrm{H} 5 \mathrm{~F} 3 \mathrm{O} 3 \mathrm{~S} 1$

0,1

O,0.8873585081,2.2336062131,-3.2255229706

C, $0.5621635846,2.0257322293,-1.9194094901$

C, $-0.1435557515,1.7542418171,0.7868732188$

C, $0.7545173764,0.8025263625,-1.2792113494$

C, $0.0203652829,3.1065062451,-1.2153874099$

C,- $0.3254047319,2.967769434,0.1232382576$

C,0.3922783682,0.6942066105,0.0629163894

$\mathrm{H}, 1.1889199428,-0.0459465197,-1.7957889863$

$\mathrm{H},-0.1170131785,4.0470649208,-1.7347554002$

$\mathrm{H},-0.7361604079,3.8149943786,0.6592319731$

$\mathrm{S}, 0.6331805789,-0.88382588,0.8784148793$

$\mathrm{H},-0.393938628,1.6356746985,1.8324476353$

O,1.6161798562,-1.6661859894,0.1357970559 
O,0.714409262,-0.6819796516,2.319945958

C,-1.0319053651,-1.7640017264,0.5837937197 $\mathrm{H}, 1.2509048842,1.4307309487,-3.6148532975$

$F,-1.2606618363,-1.8923326832,-0.7271558141$

F,-2.030107891,-1.0652393092,1.1300744813

F,- $0.9801119915,-2.9698407584,1.1415746265$

$H F=-1193.3256746 \quad$ NImag $=0$

\section{3-Tf- $\mathrm{C}_{6} \mathrm{H}_{4} \mathrm{O}^{-}$}

Brutoformula: C7H4F3O3S1(1-)

$-1,1$

$0,0.8532803024,2.1874836656,-3.2659181044$

C, $0.567185037,2.0591055813,-2.0467638719$

C, $-0.0975664483,1.7772489361,0.785612777$

C, $0.769520188,0.8213236565,-1.3195220651$

$\mathrm{C}, 0.010702873,3.1263718789,-1.2410171264$

C, $-0.3001061338,2.9820373496,0.1012528218$

C, $0.4409851706,0.7286574041,0.026086695$

$\mathrm{H}, 1.1919335069,-0.0224145898,-1.8502919698$

$\mathrm{H},-0.1586776881,4.0744234295,-1.743043758$

$\mathrm{H},-0.7093741752,3.8295759178,0.6462264408$

S, $0.6530290161,-0.8397352246,0.8353913335$

$\mathrm{H},-0.3211871203,1.6568578099,1.8355318476$

$0,1.6233863823,-1.689404637,0.1407096225$

$0,0.7116790837,-0.6868335252,2.2935019123$

C,-1.0101837128,-1.73366154,0.5502667483

F,-0.9516645941,-2.9495754832,1.1207179562

$F,-1.2684675386,-1.8945509353,-0.7522732966$

F,-2.0288841752,-1.0624449816,1.1052745391

$H F=-1192.7935202 \quad \mathrm{NImag}=0$

\section{3- $\mathrm{NO}_{2}-\mathrm{C}_{6} \mathrm{H}_{4} \mathrm{OH}$}

Brutoformula: $\mathrm{C} 6 \mathrm{H} 5 \mathrm{~N} 1 \mathrm{O} 3$

0,1

O,-1.5818316511,0.,-2.6150597353

C,-0.6159300071,0.,-1.6796603794

C, $1.4953375855,0 ., 0.1533591894$

C, $-0.8879856311,0 .,-0.3203805828$

C, $0.7052053134,0 .,-2.1218276463$

C, $1.7451933967,0 .,-1.209841517$

C, $0.1761512864,0 ., 0.5616202015$

$\mathrm{H},-1.9054180675,0 ., 0.0850771281$

$\mathrm{H}, 0.8911778571,0 .,-3.1989352322$

$\mathrm{H}, 2.77659021,0 .,-1.5712115415$

$\mathrm{N},-0.1211604653,0 ., 1.9905382116$

$\mathrm{H}, 2.2854455458,0 ., 0.9066459185$

$\mathrm{H},-2.4572037321,0 .,-2.1891476816$

O,-1.2979981998,0.,2.3194607964

$0,0.8235423235,0 ., 2.7623724807$

$H F=-512.1217486 \quad \mathrm{~N} I m a g=0$

\section{3- $\mathrm{NO}_{2}-\mathrm{C}_{6} \mathrm{H}_{4} \mathrm{O}^{-}$}

Brutoformula: $\mathrm{C} 6 \mathrm{H} 5 \mathrm{~N} 1 \mathrm{O} 3$
0,1

O,-1.5818316511,0.,-2.6150597353

C,-0.6159300071,0.,-1.6796603794

$\mathrm{C}, 1.4953375855,0 ., 0.1533591894$

C,- $0.8879856311,0 .,-0.3203805828$

C, $0.7052053134,0 .,-2.1218276463$

C, $1.7451933967,0 .,-1.209841517$

C, $0.1761512864,0 ., 0.5616202015$

$\mathrm{H},-1.9054180675,0 ., 0.0850771281$

$\mathrm{H}, 0.8911778571,0 .,-3.1989352322$

$\mathrm{H}, 2.77659021,0 .,-1.5712115415$

$\mathrm{N},-0.1211604653,0 ., 1.9905382116$

$\mathrm{H}, 2.2854455458,0 ., 0.9066459185$

$\mathrm{H},-2.4572037321,0 .,-2.1891476816$

O,-1.2979981998,0.,2.3194607964

$0,0.8235423235,0 ., 2.7623724807$

$H F=-511.582118 \quad N I m a g=0$

\section{2,4- $\left(\mathrm{NO}_{2}\right)_{2}-\mathrm{C}_{6} \mathrm{H}_{3} \mathrm{OH}$}

Brutoformula: $\mathrm{C} 6 \mathrm{H} 4 \mathrm{~N} 2 \mathrm{O} 5$

0,1

$\mathrm{N},-0.4067867862,-0.0156793873,2.749095357$

C, $0.152057468,-0.0066933804,1.3852355838$

C, $1.229708564,0.0096965309,-1.1924835992$

C, $-0.7097165498,-0.0034576372,0.3084527249$

C, $1.5424565529,-0.0020273291,1.2118075534$

C, $2.0679685555,0.0061885164,-0.0617784034$

C,- $0.1719986522,0.0051781257,-0.9777702156$

$\mathrm{H},-1.7809290103,-0.0072325882,0.4507651473$

$\mathrm{H}, 2.1838560749,-0.0048594871,2.0826227798$

$\mathrm{H}, 3.1373259424,0.0099380524,-0.2293448323$

$\mathrm{N},-1.101731835,0.0080556239,-2.1025965807$

$0,1.8078661731,0.017461299,-2.3886673907$

O,-0.6167246122,0.0144842272,-3.2494779414

$0,-2.2969836044,0.0042676259,-1.8825910645$

$0,0.3883502819,-0.0186183817,3.6807233936$

O,-1.6248384388,-0.0195883939,2.8644834167

$\mathrm{H}, 1.0951633138,0.0181603964,-3.0660797017$

$\mathrm{HF}=-716.6879285 \mathrm{~N} I \mathrm{mag}=0$

\section{2,4- $\left(\mathrm{NO}_{2}\right)_{2}-\mathrm{C}_{6} \mathrm{H}_{3} \mathrm{O}^{-}$}

Brutoformula: $\mathrm{C} 6 \mathrm{H} 3 \mathrm{~N} 2 \mathrm{O} 5(1-)$

$-1,1$

$\mathrm{N},-0.3902050712,0.0293659309,2.6753994415$

C, $0.1668582963,0.0160356267,1.3617561119$

C, $1.3036449911,-0.0194346122,-1.2944645924$

C, $-0.6728121635,0.0388111903,0.249382301$

C, $1.5746078842,-0.0319215013,1.1843174021$

C,2.1049924685,-0.0461677673,-0.0704712889

C, $-0.1447366302,-0.0088394491,-1.0265386792$

$\mathrm{H},-1.7441657847,0.0797006407,0.3845517145$

$\mathrm{H}, 2.2051552387,-0.0534860087,2.0637559492$

$\mathrm{H}, 3.1786471208,-0.0725254936,-0.2208338141$

$\mathrm{N},-1.0998223315,-0.0223556628,-2.1213451347$ 
$0,1.8287809601,0.0295333025,-2.4138757253$ $0,-0.7929537123,-0.592569455,-3.1638585474$ $0,-2.1975723937,0.5299414441,-1.9476562947$ $0,0.3854214153,0.0090211072,3.6482825413$ O,-1.6242729986,0.062365859,2.8108903357

$H F=-716.1732419$ NImag $=0$

\section{2,4,6- $-\mathrm{Tf}_{3}-\mathrm{C}_{6} \mathrm{H}_{2}-\mathrm{OH}$}

Brutoformula: C9H3F9O7S3

0,1

O,2.2221235347,1.3225063518,0.3685929239 C, $1.1502623382,0.6024169907,0.0835678904$ C, $-1.2446196497,-0.7671014523,-0.4618049899$ C, $0.032630181,1.2647305004,-0.4707819484$ C, $1.0234340232,-0.7919420854,0.3120162445$ C, $-0.1656112146,-1.4695462582,0.0557197059$ C,-1.1499779567,0.597529976,-0.7396542898 S, $0.1115561126,3.0156962407,-0.9574896538$ S,2.3991618407,-1.7476017177, 0.9407475418 $\mathrm{H},-0.242639833,-2.5323979193,0.2546209823$ $\mathrm{H},-1.9842144123,1.1390769754,-1.1711260195$ S,-2.7722038192,-1.641906643,-0.8124348186 $0,3.3585686467,-0.7971593881,1.5246911839$ $\mathrm{O}, 1.9213050345,-2.9126755244,1.6614184411$ C,3.2345349669,-2.3951230449,-0.647476395 $0,1.3071897107,3.2671265737,-1.7423059743$ O,-1.2219591969,3.3603955526,-1.4340550504 C, $0.3009516187,3.9493319258,0.7102188852$ O,-3.5084442341,-0.9027411015,-1.8270381254 O,-2.4734473892,-3.0640073133,-0.9101162289 C,-3.7468728719,-1.4209977868,0.8105434357 $\mathrm{H}, 2.8985137407,0.7831043553,0.8323824033$ $\mathrm{F}, 1.5745017355,4.0710521619,1.050632414$ F,-0.3542814013,3.2774086542,1.6644483039 F,-0.2405199512,5.1510887803,0.5614662442 $\mathrm{F}, 3.566644069,-1.3600059807,-1.4186898556$ $F, 2.3893748872,-3.1846290294,-1.3044142123$ $F, 4.3192948769,-3.0726194635,-0.3054748206$ F,-3.9296414052,-0.1211912025,1.0520370384 F,-3.0645547586,-1.9601132626,1.8230174839 $F,-4.9207028414,-2.0245621341,0.6889068409$

$\mathrm{HF}=-2964.8409112 \mathrm{NImag}=0$

\section{2,4,6- $\mathrm{Tf}_{3}-\mathrm{C}_{6} \mathrm{H}_{2} \mathrm{O}^{-}$}

Brutoformula: $\mathrm{C9H} 2 \mathrm{~F} 9 \mathrm{O} 7 \mathrm{~S} 3(1-)$

$-1,1$

O,2.1570607239,-1.0210011653,-0.4950227092 C, $1.0121303289,-0.6290740814,-0.2616429534$ C, $-1.6685792316,0.2855035295,0.2841950476$ C, $0.0769214665,-0.1858064491,-1.2945034301$ C, $0.4330231361,-0.5582466921,1.0792854212$ C, $-0.8495976003,-0.1401081177,1.3438249688$ C,-1.1948422063,0.269054844,-1.0385355929 S, $0.5548013304,-0.3274465123,-3.0082848939$
S, 1.4614705955,-0.9495438967,2.484095599 $\mathrm{H},-1.2215599502,-0.1431611848,2.3615602814$ $\mathrm{H},-1.8312064039,0.590274369,-1.854652426$ S, $-3.2864207266,0.8653179203,0.6178189211$ $0,2.6761153843,-0.1427630763,2.5264687071$ $0,0.5932258783,-1.0322924064,3.6631511821$ C,2.0397444881,-2.7600710684,2.1956127662 $0,0.9342866402,-1.6881023859,-3.3723391416$ O,-0.437402243, $0.3981222799,-3.8082171455$ C,2.1537404351,0.7276322284,-3.1709178996 O,-4.1016260742,0.8229123099,-0.5954208004 O,-3.7661720063,0.3334874949,1.8926916567 C,-3.0573352798,2.7254993564,0.955253306 F,-4.2457001379,3.2785734422,1.2237388261 F,3.2515326035,0.0005699516,-3.0379046169 $F, 2.1550343572,1.7169368606,-2.2702126518$ $F, 2.1510664158,1.2724967222,-4.3977720944$ F,3.2170606067,-2.8216569164,1.5939955317 $\mathrm{F}, 1.1310729977,-3.4313981539,1.4792424636$ $F, 2.1461926366,-3.3442182662,3.3994038223$ F,-2.5297770718,3.3395250733,-0.1105638163 F,-2.2475926835,2.919220279,2.0033052388

$$
\mathrm{HF}=-2964.3674072 \mathrm{NImag}=0
$$

\section{$\mathrm{C}_{6} \mathrm{H}_{5}-\mathrm{COOH}$}

Brutoformula: $\mathrm{C} 7 \mathrm{H} 6 \mathrm{O} 2$

0,1

$\mathrm{H}, 1.8357882962,0.0000000051,-2.2453630752$ C, $0.9629550168,-0.0000000003,-1.602690809$ C,-1.2869386847,-0.000000008,0.047989847 C, $1.1215964821,-0.0000000119,-0.2199963656$ C, $-0.3154713031,0.0000000045,-2.1602000061$ C, $-1.4399302919,0.0000000008,-1.3337318828$ C, $-0.0053631144,-0.0000000153,0.6110013069$ $\mathrm{H}, 2.1104303253,-0.0000000155,0.2192583076$ $\mathrm{H},-0.435616767,0.0000000166,-3.2378102221$ $\mathrm{H},-2.4333362364,0.0000000064,-1.7671975037$ C, $0.1071636434,-0.0000000251,2.0934656386$ $\mathrm{H},-2.1458179112,-0.0000000065,0.7075776088$ O,- $0.8271239759,0.0000000121,2.8609984259$ $0,1.393107146,0.0000000105,2.5296460216$ $\mathrm{H}, 1.3585573749,0.0000000267,3.4970527091$

$\mathrm{HF}=-420.9481333 \mathrm{NImag}=0$
$\mathrm{C}_{6} \mathrm{H}_{5}-\mathrm{COO}^{-}$
Brutoformula: $\mathrm{C} 7 \mathrm{H} 5 \mathrm{O} 2(1-)$
$-1,1$
$\mathrm{H},-2.1484626884,-0.0000000108,-1.7788563261$
C,-1.2055769411,-0.0000000038,-1.2378126958
C, $1.2012785616,0.0000000189,0.1571714483$
C, $-1.2012785719,0.0000000213,0.1571714521$
C, $0.0000000015,-0.0000000112,-1.9431263123$
C, $1.2055769347,-0.0000000004,-1.237812687$
C, $-0.0000000016,0.0000000306,0.8743748403$ 
$\mathrm{H},-2.1215017275,0.0000000293,0.7309099017$ $\mathrm{H}, 0.000000008,-0.0000000283,-3.029434359$ $\mathrm{H}, 2.1484626853,-0.0000000065,-1.7788563057$ C, $0.0000000164,0.0000000549,2.4286135359$ $\mathrm{H}, 2.1215017097,0.0000000237,0.7309098936$ $0,1.1335197643,-0.0000000581,2.9633738652$ $0,-1.1335197509,-0.0000000595,2.9633738498$

$\mathrm{HF}=-420.3966976 \mathrm{NImag}=0$

\section{$\mathrm{C}_{6}\left(\mathrm{CF}_{3}\right)_{5}-\mathrm{O}-\mathrm{O}-\mathrm{C}_{6}\left(\mathrm{CF}_{3}\right)_{5}$}

Brutoformula: $\mathrm{C} 22 \mathrm{~F} 30 \mathrm{O} 2$

0,1

$0,0.2909112684,0.7106841093,-1.41428497$

O,-0.2909112684,-0.7106841093,-1.41428497

C,-1.5230599869,-0.6345652775,-0.8296742312

C, $1.5230599869,0.6345652775,-0.8296742312$

C,-3.9673821849,-0.4981919345,0.4902041636

C,-2.5604001461,0.1118863095,-1.4088171934

C,-1.6898796631,-1.3334428008,0.3776087262

C,-2.8913063885,-1.1982145487,1.0836295705

C,-3.770957832,0.2350961078,-0.700878332

C,-2.3563501687, $0.6663174246,-2.8361977086$

C,- $-0.5434987466,-2.283686141,0.7695568868$

C,-2.9484577272,-1.6239764218,2.570553274

C,- $4.8165499184,1.2877835848,-1.1467339085$

C, $-5.3990030865,-0.7097618326,1.0438814682$

C, $3.9673821849,0.4981919345,0.4902041636$

C, $1.6898796631,1.3334428008,0.3776087262$

C, $2.5604001461,-0.1118863095,-1.4088171934$

C,3.770957832,-0.2350961078,-0.700878332

C,2.8913063885,1.1982145487,1.0836295705

C, $0.5434987466,2.283686141,0.7695568868$

C, $2.3563501687,-0.6663174246,-2.8361977086$

C,4.8165499184,-1.2877835848,-1.1467339085

C,2.9484577272,1.6239764218,2.570553274

C,5.3990030865,0.7097618326,1.0438814682

$F, 3.2614182462,2.9077651886,2.7667194678$

$F, 3.8267655789,0.8694700112,3.2492232746$

F,1.749501053,1.3832158698,3.1408191587

$\mathrm{F}, 6.2828801582,0.7074716079,0.0287172747$

$F, 5.8023040462,-0.1844569838,1.9483552192$

$F, 5.4864863876,1.9354687214,1.5985673149$

$\mathrm{F}, 4.1874288241,-2.3542717574,-1.6728113357$

$F, 5.4957548754,-1.7554483992,-0.0852311312$

$F, 5.7006038142,-0.8365316134,-2.0411482751$

$F, 1.5463944353,0.1331654987,-3.5460783731$

$F, 1.8347752426,-1.899218401,-2.8499639299$

$F, 3.5241114103,-0.6912884533,-3.5037451532$

$\mathrm{F}, 0.118215194,2.9559774832,-0.3166108633$

$F, 0.9124149586,3.2155143769,1.6529046524$

F,-0.5127836659,1.615324412,1.2786697509

F, $-0.118215194,-2.9559774832,-0.3166108633$

F,-0.9124149586,-3.2155143769,1.6529046524

$F, 0.5127836659,-1.615324412,1.2786697509$

F,-3.8267655789,-0.8694700112,3.2492232746
F,-1.749501053,-1.3832158698,3.1408191587

F,-3.2614182462,-2.9077651886,2.7667194678

F,-5.4864863876,-1.9354687214,1.5985673149

F,-6.2828801582,-0.7074716079,0.0287172747

$F,-5.8023040462,0.1844569838,1.9483552192$

$F,-5.4957548754,1.7554483992,-0.0852311312$

$F,-5.7006038142,0.8365316134,-2.0411482751$

F,-4.1874288241,2.3542717574,-1.6728113357

F,-3.5241114103,0.6912884533,-3.5037451532

$F,-1.5463944353,-0.1331654987,-3.5460783731$

$F,-1.8347752426,1.899218401,-2.8499639299$

$\mathrm{HF}=-3985.093243 \quad \mathrm{NImag}=0$ 